\title{
Quantitative methods for improved error detection in dose-guided radiotherapy
}

Citation for published version (APA):

Wolfs, C. (2020). Quantitative methods for improved error detection in dose-guided radiotherapy.

[Doctoral Thesis, Maastricht University]. Ipskamp Printing BV. https://doi.org/10.26481/dis.20200925cw

Document status and date:

Published: 01/01/2020

DOI:

$10.26481 /$ dis.20200925cw

Document Version:

Publisher's PDF, also known as Version of record

\section{Please check the document version of this publication:}

- A submitted manuscript is the version of the article upon submission and before peer-review. There can be important differences between the submitted version and the official published version of record.

People interested in the research are advised to contact the author for the final version of the publication, or visit the DOI to the publisher's website.

- The final author version and the galley proof are versions of the publication after peer review.

- The final published version features the final layout of the paper including the volume, issue and page numbers.

Link to publication

\footnotetext{
General rights rights.

- You may freely distribute the URL identifying the publication in the public portal. please follow below link for the End User Agreement:

www.umlib.nl/taverne-license

Take down policy

If you believe that this document breaches copyright please contact us at:

repository@maastrichtuniversity.nl

providing details and we will investigate your claim.
}

Copyright and moral rights for the publications made accessible in the public portal are retained by the authors and/or other copyright owners and it is a condition of accessing publications that users recognise and abide by the legal requirements associated with these

- Users may download and print one copy of any publication from the public portal for the purpose of private study or research.

- You may not further distribute the material or use it for any profit-making activity or commercial gain

If the publication is distributed under the terms of Article $25 \mathrm{fa}$ of the Dutch Copyright Act, indicated by the "Taverne" license above, 


\section{Quantitative methods for improved error detection in dose-guided radiotherapy}

Cecile J.A. Wolfs 


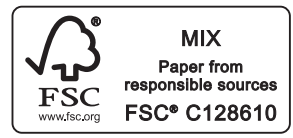

Printed by: Ipskamp Printing, Enschede ISBN: 978-94-028-2130-7

(c) Copyright C.J.A. Wolfs, Maastricht 2020

All rights reserved. No part of the material protected by this copyright notice may be reproduced or utilised in any form or by any means, electronic or mechanical, including photocopying, recording or by any information storage and retrieval system, without written permission from the author and the publisher(s) holding the copyrights of the reprinted article(s). 


\title{
Quantitative methods for improved error detection in dose-guided radiotherapy
}

\author{
PROEFSCHRIFT \\ ter verkrijging van de graad van doctor aan de Universiteit Maastricht, \\ op gezag van de Rector Magnificus, Prof. dr. Rianne M. Letschert, \\ volgens het besluit van het College van Decanen, \\ in het openbaar te verdedigen \\ op vrijdag 25 september 2020 om 10.00 uur
}

door

Cecile Julie Andrée Wolfs 


\section{Promotor}

Prof. dr. ir. F.J.W. Verhaegen

\section{Co-promotores}

Dr. ir. S.M.J.J.G. Nijsten

Dr. G. Paiva Fonseca

\section{Beoordelingscommissie}

Prof. dr. ir. A.L.A.J. Dekker (voorzitter)

Prof. dr. D. De Ruysscher

Prof. dr. E.C. Ford (University of Washington, Seattle, WA, USA)

Prof. dr. G. Landry (Medical Center of the University of Munich, Munich, Germany) 
Veur opa 



\section{Table of contents}

Page

Introduction

Chapter 1 Introduction and outline of the thesis

Part I: Uncertainty and sensitivity of portal dosimetry methods

Chapter 2 Validation and uncertainty analysis of a pre-treatment 2D dose 17 prediction model

Chapter 3 Should dose from small fields be limited for dose verification procedures?: Uncertainty vs. small field dose in VMAT treatments

Chapter 4 Detection of anatomical changes in lung cancer patients with 2D time-integrated, 2D time-resolved and 3D time-integrated portal dosimetry: A simulation study

Part II: Advanced methods for error classification using portal dosimetry

Chapter 5 Deep learning for error detection in EPID dosimetry: A proof of concept

Chapter 6 Deep learning for in vivo EPID dosimetry classification: Relating gamma analysis and dose-volume histograms

Chapter 7 Detection of treatment error types using convolutional neural networks and EPID dosimetry

Chapter 8 External validation of a hidden Markov model for gamma-based classification of anatomical changes in lung cancer patients using EPID dosimetry

\section{Discussion}

Chapter 9 Discussion and future perspectives

\section{Appendices}

Summary

Samenvatting

Valorization Addendum

Curriculum Vitae \& List of publications

Dankwoord 



\section{Chapter 1}

Introduction and outline of the thesis 



\section{Cancer}

Every hour, 13 people in the Netherlands are diagnosed with cancer ${ }^{1}$. Globally, there were 18.1 million new cases of cancer in 2018, and 9.6 million cancer related deaths $\mathbf{s}^{2,3}$. Even though treatment options have evolved and improved substantially over the years, these numbers show that cancer is still a prominent disease, and a leading cause of death worldwide ${ }^{4}$. With a predicted rise to 24.1 million new cases and 13 million deaths in 2030 (i.e., a nearly 35\% increase in approximately 10 years), cancer is expected to remain a major health problem in future years as well ${ }^{5}$.

\section{Radiotherapy}

Next to systemic treatment and surgery, radiation therapy or radiotherapy is one of the main treatment options for many cancer types, and it can benefit approximately $50 \%$ of all cancer patients ${ }^{6}$. In radiotherapy, ionizing radiation is used to break the DNA strands in cancer cells, resulting in death of those cells. Different forms of radiotherapy treatment exist, with the two main categories being external beam radiotherapy (EBRT) and brachytherapy. In EBRT, beams of radiation are aimed at the tumor from outside the body, while in brachytherapy the radiation is delivered from inside the body, for instance using radioactive seeds. Nowadays, the majority of EBRT treatments is performed with photon beams, although the availability of EBRT with proton and carbon ion beams is growing rapidly?

The focus of this thesis is on photon EBRT. This type of EBRT is generally delivered using a linear accelerator (linac) that produces high-energy megavolt (MV) photon beams. Over the years, treatment delivery using linacs has become more precise and complex, with the aim of delivering a high radiation dose to the tumor and as little dose as possible to surrounding healthy tissues. This trend started with 3D conformal radiotherapy (3D-CRT), where multiple radiation beams are aimed at the tumor from different angles. These beams are shaped to conform to the shape of the tumor. The next step, developed in the early to mid 1990s, was intensity modulated radiotherapy ${ }^{8}$ (IMRT), in which multiple beams from different angles are used as well, but the intensity and shape of the beam can be modulated during delivery, using a multi-leaf collimator (MLC). An MLC consists of leaves of a high density material, that can open and close independently, also during irradiation. Volumetric modulated arc therapy ${ }^{9,10}$ (VMAT), introduced in 2007, adds even more degrees of freedom by exploiting linac rotation while irradiating the patient. Hence, the radiation is delivered from many angles, which means that the high dose can be aimed more specifically at the target volume, while the low dose is smeared out over a larger volume. Although VMAT can be beneficial in terms of improved target coverage and lower treatment time, the spread of the low dose radiation over a large normal tissue volume remains a source of concern, as this might increase the risk of secondary malignancies ${ }^{10}$.

\section{Radiotherapy workflow}

After a patient is diagnosed with cancer and referred for EBRT, the EBRT workflow (Figure 1.1) begins with the treatment preparation phase. The first step in this phase is to obtain a 3-dimensional (3D) or, for tumor sites where motion plays a role, a 4-dimensional (4D) image to visualize the patient's anatomy. The most common image 
acquisition method in radiotherapy is computed tomography (CT), although positron emission tomography (PET) and magnetic resonance imaging (MRI) can be useful for visualizing anatomy that is difficult to distinguish on CT scans. On these scans, the tumor and relevant healthy tissues (organs-at-risk: OARs) are contoured. For the tumor, three volumes are defined. First, the gross tumor volume (GTV) is delineated, which comprises the visible tumor. Second, a margin is added to the GTV to include microscopic disease, constituting the clinical target volume (CTV). Third, the planning target volume (PTV) is established by adding an additional margin to the CTV, to compensate for uncertainties in treatment delivery, for instance, patient positioning or organ motion ${ }^{11}$.

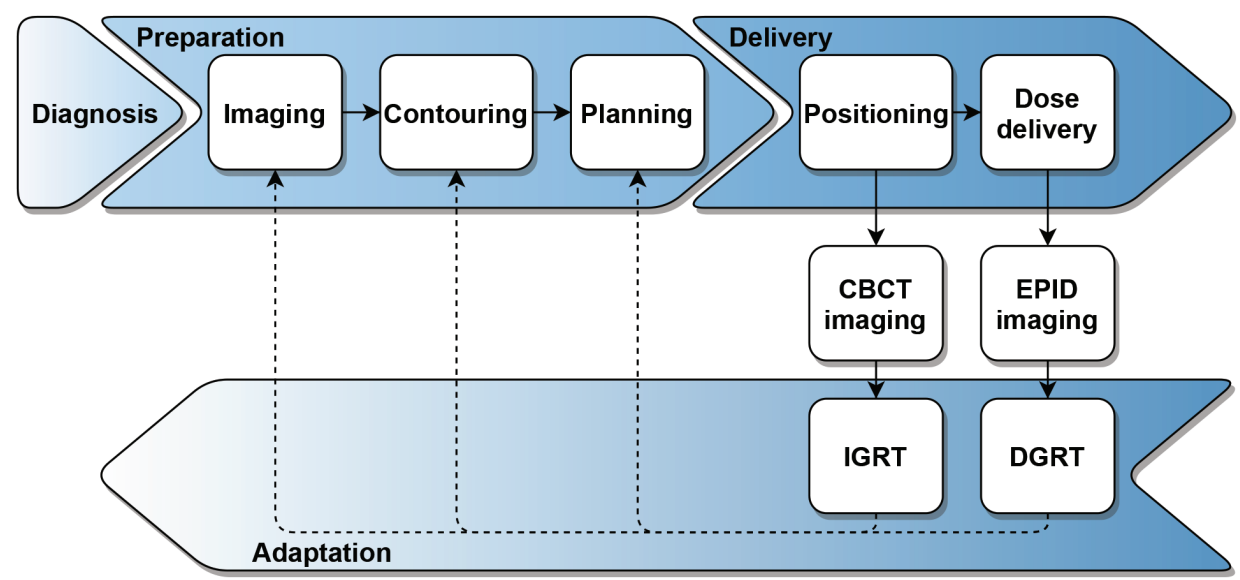

Figure 1.1: The EBRT workflow. In the preparation phase, the patient's anatomy is visualized, relevant structures are contoured and a treatment plan is made. In the delivery phase, the patient is positioned in the same way as in the preparation phase, and the dose is delivered according to the treatment plan. An adaptive feedback loop can be initiated if there are large differences observed in the images obtained for positioning or in the delivered dose. CBCT: cone beam computed tomography, EPID: electronic portal imaging device, IGRT: image-guided radiotherapy, DGRT: dose-guided radiotherapy.

In the treatment planning system (TPS), a treatment plan is made by placing lower and upper limits on the dose that the tumor and OARs should receive, respectively. This process is called inverse planning, as the intensity and shape of the radiation beams are determined by the dose limits in a mathematical optimization process, instead of directly by a treatment planner ${ }^{12}$. The dose limits are formulated in the form of dosevolume histogram (DVH) metrics. A DVH curve is a graphical representation of the dose distribution that a certain structure receives ${ }^{13}$. From DVHs, metrics can be calculated, which are used to guide the inverse planning process and are the main clinical tool to assess the quality of the treatment plan and corresponding dose distribution. DVH metrics can be simply a mean or maximum dose in a structure, but they can also represent the minimum dose that a certain volume of a structure receives, and vice versa.

In the delivery phase, the patient undergoes multiple (daily) fractions of radiotherapy. Due to the high precision of the treatment and its specificity to the 
patient's anatomy, it is important that the patient is positioned in the same manner as in the imaging step in the preparation phase, to ensure the radiation dose is delivered to the correct location. For this purpose, most modern linacs are equipped with systems for positioning verification. One such system is the electronic portal imaging device $(E P I D)^{14}$. The EPID is a flat panel imaging detector, located opposite the linac head. This means that the EPID images the MV photon beam produced by the linac, behind the patient. These images are two-dimensional (2D), and due to the high energy of the beam the image contrast is low. They are therefore mostly useful for visualizing bony anatomy and metal markers. In recent years, the EPID has been replaced for this purpose by cone beam CT (CBCT) systems. These also consist of a flat panel detector but use a separate kilovolt $(\mathrm{kV}) \mathrm{X}$-ray source ${ }^{15}$, which offers higher image contrast. A CBCT system can additionally provide a 3D image of the patient, meaning that it can be compared with the planning $C T$ ( $\mathrm{pCT}$ ) scan to check for positioning errors. After the position is matched, the radiation is delivered as specified in the treatment plan.

\section{Treatment verification and adaptive radiotherapy}

With the increasing complexity of radiotherapy, it has become increasingly important to verify that the desired dose is delivered as planned. As described above, one way to ensure this is by correct patient positioning. However, other deviations can occur, such as errors in transfer of the treatment plan from the TPS to the linac or anatomical changes in the patient, for instance, tumor shift (Figure 1.2), weight loss or organ motion. In the case of anatomical changes, a distinction can be made between interand intrafraction changes. Interfraction changes occur between fractions (e.g., tumor shift or tumor regression), while intrafraction changes occur within a fraction (e.g., breathing motion). This thesis focuses on interfraction changes.

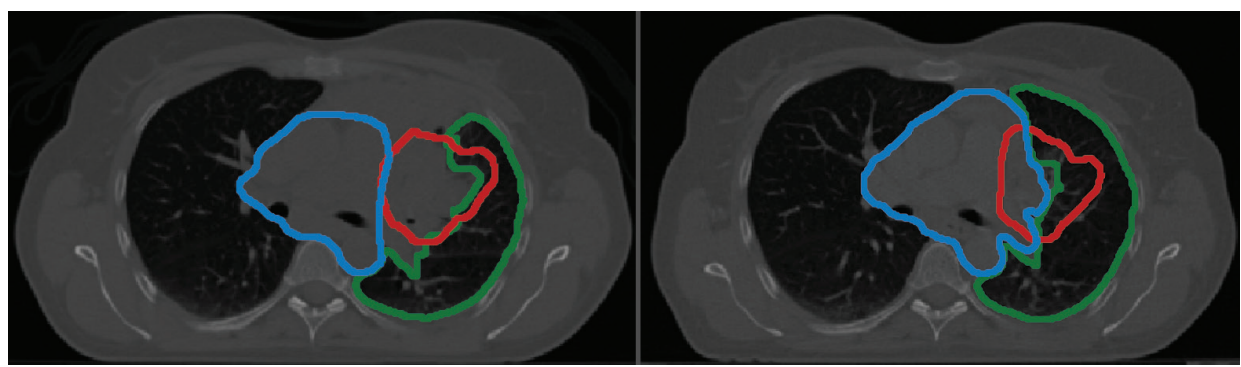

Figure 1.2: Axial slice of two CT scans taken at different time points of the same lung cancer patient, with the CTV delineated in red, the left lung in green and the mediastinum in blue. The CT scan on the left is the original planning $\mathrm{CT}$, the one on the right shows anatomical changes (shift of CTV and mediastinum) due to a decrease of atelectasis in the left lung.

Different strategies have been developed to detect different types of errors. However, a commonality of these strategies is that they can be included in the radiotherapy workflow by adding an adaptive feedback loop. The purpose of this adaptive radiotherapy loop is to ensure quality of treatment, by adapting the treatment if a persistent error has occurred $^{16}$. Treatment adaptation can take different forms: from changing the number of fractions in the treatment to completely repeating the preparation phase. It is important to have a robust methodology for detecting 
changes, to avoid flagging or re-planning patients that do not require adaptation after all (false positives), which would unnecessarily increase workload.

The two main strategies for treatment verification and adaptive radiotherapy are image-guided radiotherapy (IGRT) and dose-guided radiotherapy (DGRT) (Figure 1.1). In IGRT, some form of imaging is used to visualize the patient's anatomy from day to day, and to assess if it has substantially changed from the PCT. Generally, the imaging technique used for IGRT is CBCT, as this is widely available as a standard component of the linac, and is easy to compare with the PCT. However, this method does not provide information about the quantitative impact of visible anatomical changes on the planned dose distribution. This is where DGRT can be useful, as DGRT uses dose recalculation to assess if the delivered dose distribution corresponds to the planned dose distribution.

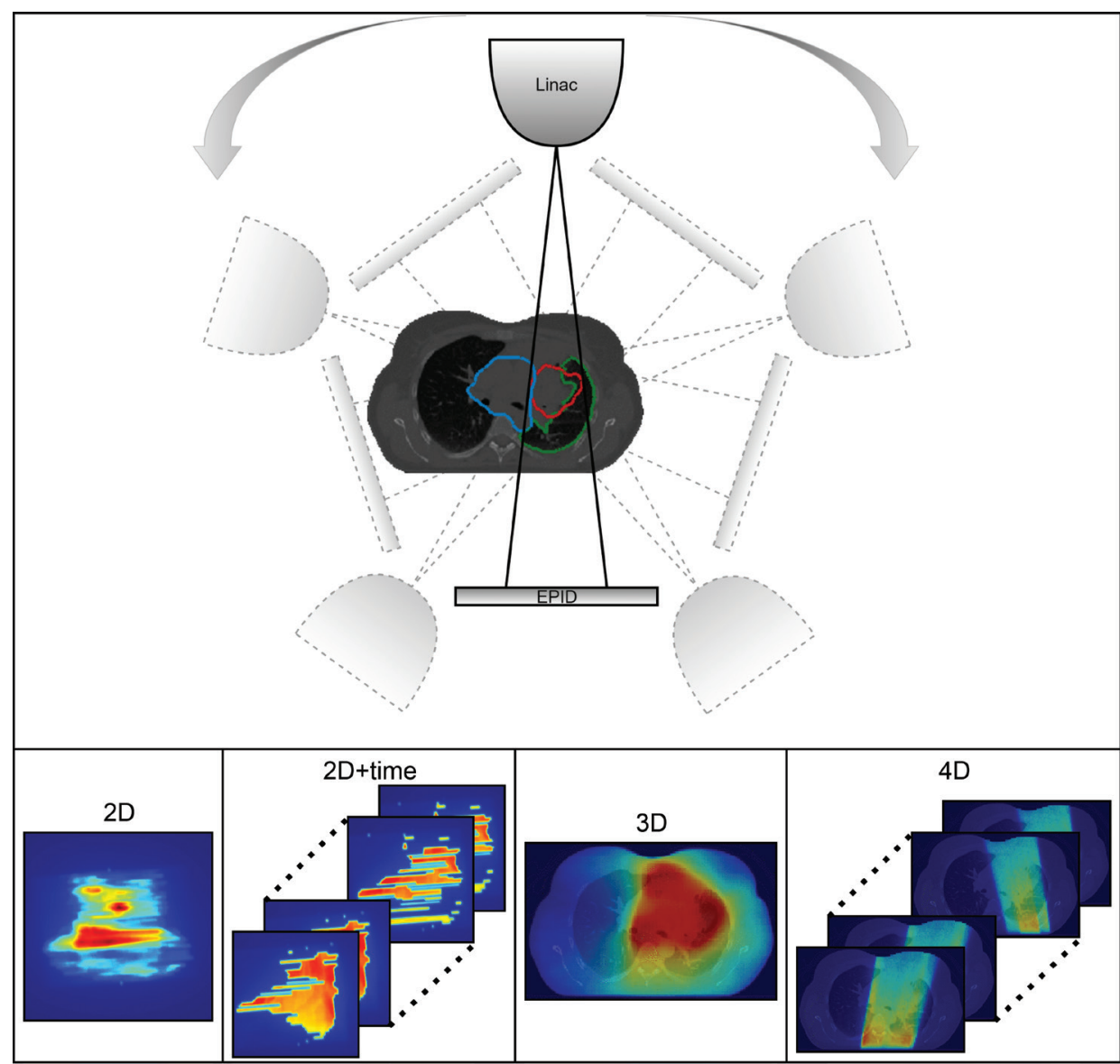

Figure 1.3: The portal dosimetry process. The top panel shows schematically how the EPID images the treatment beam while the patient is being treated. The bottom panels show the different dose distributions that the EPID images can be converted to. This process can also be performed without a patient in the beam for pre-treatment verification. In that case, the planning CT scan is used to calculate the 3D and 4D doses. 


\section{Portal dosimetry}

Although the EPID was originally used for patient positioning, the fact that it images the MV treatment beam can be exploited for dosimetric purposes, which makes it suitable for DGRT. Over the years, several calibration and calculation models have been developed, that allow for conversion of EPID images into 2D, 3D or even 4D doses ${ }^{17-19}$. This process is called EPID dosimetry or portal dosimetry, and is demonstrated in Figure 1.3. The general portal dosimetry workflow involves a dose prediction ${ }^{20,21}$, dose measurement ${ }^{17-19}$ and comparison of the predicted and measured doses. This comparison is usually performed using gamma analysis ${ }^{22-24}$, as illustrated in Figure 1.4.

Portal dosimetry can be used in different stages of the radiotherapy workflow. After the preparation phase, but before the delivery phase, the treatment plan is irradiated once without the patient present, directly onto the EPID. This pre-treatment verification is performed to confirm correct transfer of the treatment plan from the TPS to the linac. Errors that can occur here are, for instance, errors in beam shaping (i.e., MLC errors) and linac output ${ }^{25}$. During patient irradiation, the EPID can be used to perform transit portal dosimetry. The EPID then images the exit radiation while the patient is being treated and from these images 2D, 3D or 4D dose distributions can be derived. This means that with this method it is also possible to detect patient-related errors, such as anatomical changes or positioning errors ${ }^{25}$.

Several commercial software packages are available for clinical implementation of portal dosimetry ${ }^{26}$. The capabilities of such packages are usually limited to 2D or 3D verification. At institutes with in-house developed systems, verification in $2 \mathrm{D}+$ time or $4 \mathrm{D}$ is also available ${ }^{19,27,28}$, although often for research purposes only.

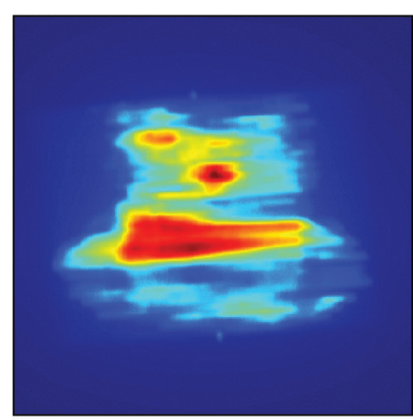

Reference

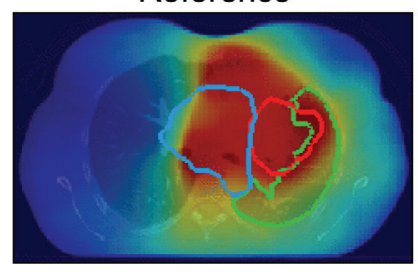

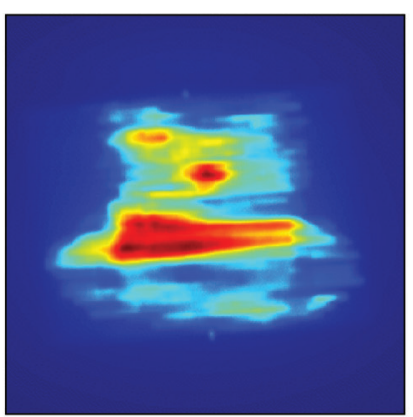

Acquired

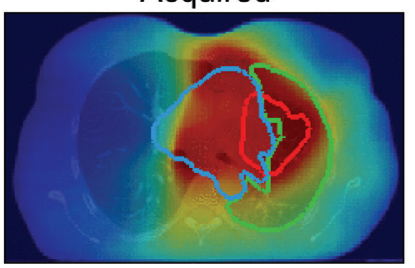

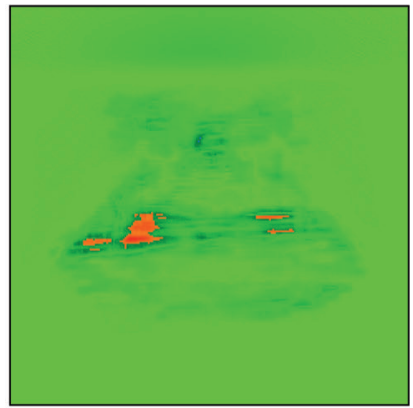

Comparison

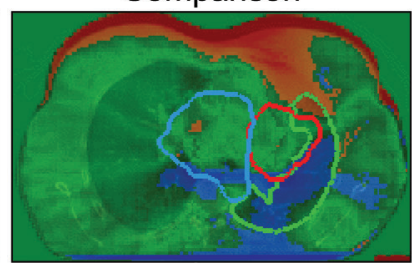

Figure 1.4: Examples of 2D (top) and 3D (bottom) dose distribution comparison using gamma analysis. An acquired dose (e.g., an EPID measurement) is compared with a reference dose (e.g., the planned dose). In the dose distributions, red indicates high dose, while blue indicates low dose. In the comparison, red regions indicate overdosage, and blue regions underdosage. 


\section{Error detection and decision making in adaptive radiotherapy}

With portal dosimetry for DGRT, the aim is to flag patients for whom relevant dosimetric errors occur, and to adapt their treatment plan. However, automatic patient selection for adaptive radiotherapy using portal dosimetry remains difficult. One reason for this is the unknown uncertainty of the portal dosimetry methods. In natural sciences and engineering it is common to report an uncertainty whenever a measurement is performed. This uncertainty expresses the dispersion of values that the measurement could have taken ${ }^{29}$. In other words, the uncertainty gives an indication of how certain one is about the measured value. When taking a decision for adaptive radiotherapy based on a portal dose measurement, the measurement uncertainty should be known and not exceed the action threshold. Otherwise, when a deviation between dose measurement and prediction is observed, it will be impossible to determine if an actual error has occurred, or if it was due to the inherent spread of the measurement. In the field of radiotherapy, such uncertainties are mostly unknown and unexplored.

Another factor that provides difficulties for the development of decision protocols is the lack of objective triggers for adaptation. In the current clinical process, if a new treatment plan is made, the dose distributions for the current and new treatment plan are compared on the basis of DVH curves and metrics. In Figure 1.5, it is clearly visible that the DVH curves have changed after recalculating the dose on the new CT, i.e., on the changed anatomy. The dose to the target volume has decreased, while the dose to the OARs has increased. However, there are no objective action thresholds for determining when these deviations are too large, and the decision when and how to adapt treatment is left to the radiation oncologist. This means that there are also no objective decision criteria for dose distributions obtained through portal dosimetry. Moreover, DVH analysis is only possible for 3D dose distributions, while portal dosimetry can also provide 2D or 4D doses. In these cases, gamma analysis is used for dose comparison, but it remains difficult to relate gamma analysis results to DVH analysis results ${ }^{30-32}$.

Lastly, portal dosimetry methods produce a vast amount of data. EPID measurements are performed routinely in the clinic, which results in many 2D/3D/4D dose distributions, gamma analysis results and DVH analysis results that are produced and saved daily. However, in terms of workload, it is not possible for human observers to analyze all this data in detail, and this process needs to be automated. Therefore, portal dosimetry results are often compressed into a few numbers, such as a gamma fail rate (proportion of pixels/voxels with $|\gamma|>1$ ), mean gamma value, or DVH metrics. This potentially causes a loss of information. Ideally, a model for error detection and decision making based on portal dosimetry should be able to take more information into account (e.g., full 2D or 3D information).

\section{Artificial intelligence}

Recently, artificial intelligence (AI) methods, such as machine learning, have experienced a major surge in the medical field, including radiation oncology ${ }^{33}$. In general, machine learning algorithms identify patterns in data through complex mathematical relationships, and apply these relationships to new data with the aim of making predictions or decisions, without being explicitly programmed to do 


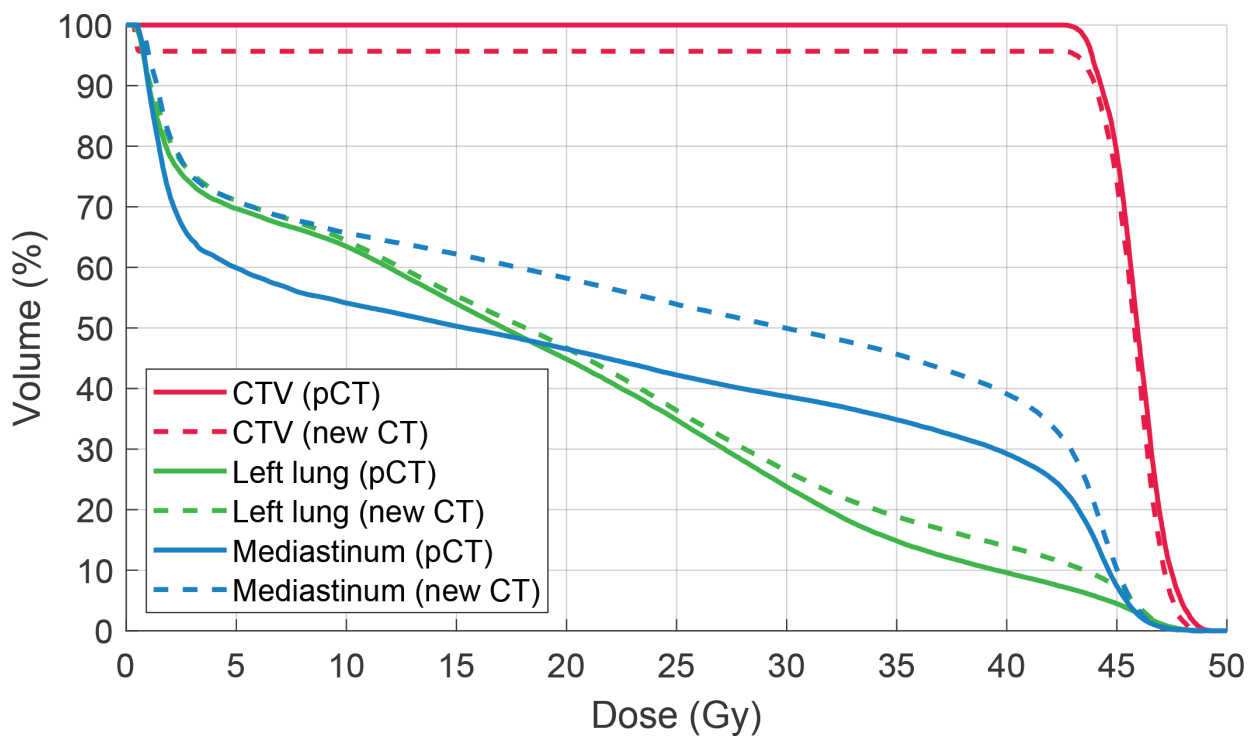

Figure 1.5: DVH curves for the CTV, left lung and mediastinum of the lung cancer patient shown in Figure 1.2, and 3D dose distributions shown in Figure 1.4. Solid curves are for the dose calculated on the planning $\mathrm{CT}$ (reference dose), dashed curves for the dose calculated on the new CT (acquired dose) at a different time point, hence including anatomical changes.

$\mathrm{so}^{34}$. In practice, a machine learning algorithm is provided with many examples in a training dataset, which is used to infer the mathematical relationships, and optimize model parameters. In supervised learning, the training process consists of learning a relationship between inputs and corresponding outputs. In unsupervised learning, the training process is aimed at finding relationships between the inputs themselves, without corresponding outputs.

Once a model is trained it can be applied to a validation or test dataset, containing examples the model has not seen before. If the model is trained well and the training data contained enough variation, it should also perform well on the validation or test data. If the model only works well on the training data, it has overfitted. To prevent poor model performance due to overfitting, the validation set can be used during the training process, to evaluate different models and hyperparameters, and to select the combination that performs best on the validation set as the final model. This process is illustrated in Figure 1.6.

Deep learning is a subset of machine learning ${ }^{35}$. In machine learning, it is necessary to create a representation of the input data by extracting relevant features. In the case of a dataset containing images, features can be seen as descriptors of the image, that are relevant for the learning process of the algorithm. In the context of portal dosimetry, metrics such as the gamma fail rate and mean gamma value can be considered features that could be used as input for machine learning algorithms. For deep learning algorithms, this manual feature extraction process is not necessary anymore, as the algorithm itself creates a representation. Considering an image dataset, this means that deep learning algorithms can take a full image (e.g., a 2D 
gamma map) or even a 3D volume as input. A disadvantage of deep learning is that, in general, there are many more parameters to train than in a machine learning model. This means that extensive amounts of training data are needed to avoid overfitting, as well as computer hardware capable of processing all this data.

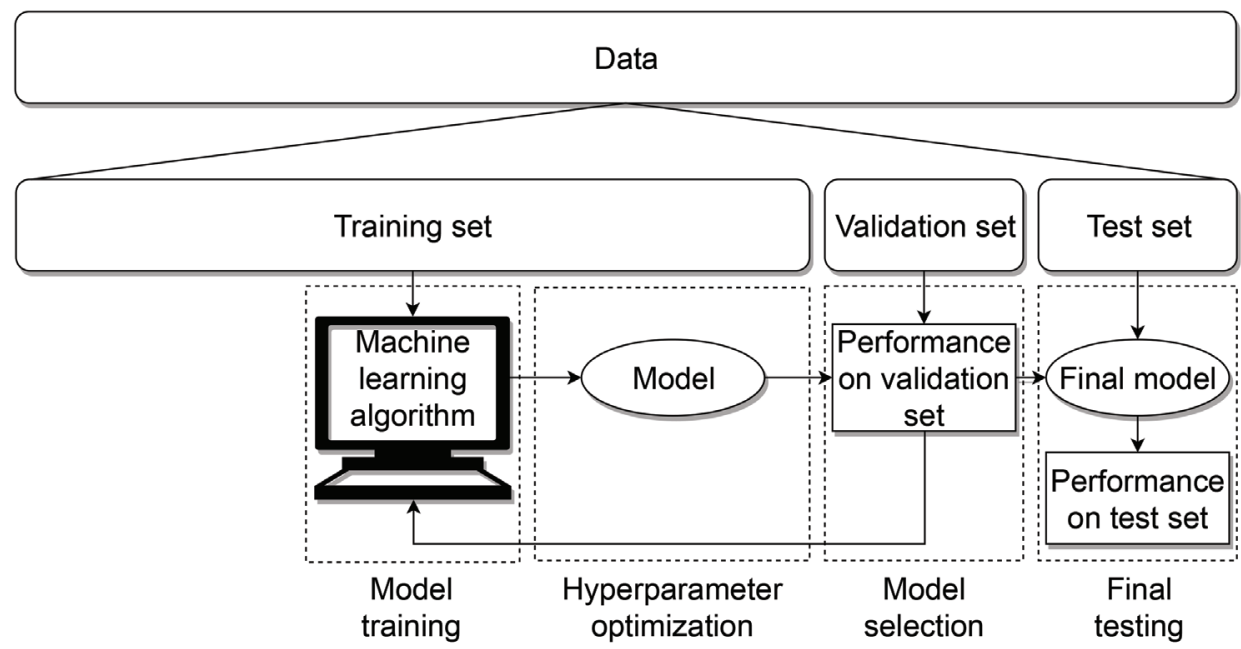

Figure 1.6: Schematic representation of the training process of a machine learning algorithm, using a validation set for hyperparameter optimization and model selection.

\section{Objectives and outline of the thesis}

The overall aim of this thesis is to investigate the error detection capabilities of portal dosimetry methods. To this end, the thesis is divided into two parts. The first part concerns the uncertainties and sensitivity of portal dosimetry methods. The objective is to establish a framework for analyzing uncertainties, and to determine which levels of anatomical change can be detected with the different portal dosimetry methods and simple decision models. The second part focuses on advanced methods for error detection and classification, applying Al to portal dosimetry. The objective is to explore how the power of Al can be harnessed for evaluating portal dosimetry results, potentially overcoming some of the current difficulties in decision making for adaptive radiotherapy. The outline of this thesis is shown in Figure 1.7 and elaborated on below.

This chapter 1 provides a general introduction to radiotherapy, adaptive radiotherapy, portal dosimetry and artificial intelligence.

\section{Part I: Uncertainty and sensitivity of portal dosimetry methods}

Chapter 2 describes a 2D pre-treatment dose prediction model that can be used in a portal dosimetry workflow. This model is validated with film measurements and TPS dose calculations. Additionally, a framework for assessing the uncertainty of such a model is presented and the uncertainty is evaluated for three patient cases.

Chapter $\mathbf{3}$ shows how the uncertainty of the dose prediction model described in Chapter 2 is influenced by the presence of small radiation fields in VMAT treatments.

Chapter 4 compares the sensitivity of 2D time-integrated, 2D time-resolved and 3D 
time-integrated portal dosimetry methods to detect different levels of simulated anatomical changes in lung cancer patients, using a simple decision protocol.

\section{Part II: Advanced methods for error classification based on portal dosimetry}

Chapter 5 provides a proof of concept of a deep learning method for detecting relevant dose errors using gamma analysis based on portal dosimetry simulations.

Chapter 6 relates portal dosimetry results to DVH metrics using deep learning, using in vivo EPID measurements and dose recalculations on CBCT images.

Chapter 7 presents a sensitivity study for detecting different types of simulated errors using deep learning. It is investigated how well a deep learning method can differentiate between different types of errors, and to which level of detail errors can be detected.

Chapter 8 validates a hidden Markov model for classifying gamma analysis results of in vivo EPID measurements into different categories of anatomical change for lung cancer patients with an external dataset. Additionally, the relation between model classification and differences in DVH metrics is analyzed.

The thesis is concluded by Chapter 9, in which the work in this thesis is discussed and directions for future research are provided.

Introduction

Chapter 1: Introduction and outline of the thesis

Uncertainty and sensitivity of portal dosimetry methods

Chapter 2: Validation and uncertainty analysis of a pre-treatment 2D dose prediction model

Chapter 3: Should dose from small fields be limited for dose verification procedures?: Uncertainty vs. small field dose in VMAT treatments

Chapter 4: Detection of anatomical changes in lung cancer patients with 2D time-integrated, 2D time-resolved and 3D time-integrated portal dosimetry: A simulation study

Advanced methods for error classification based on portal dosimetry

Chapter 5: Deep learning for error classification in EPID dosimetry: A proof of concept

Chapter 6: Deep learning for in vivo EPID dosimetry classification: Relating gamma analysis and dosevolume histograms

Chapter 7: Detection of treatment error types using convolutional neural networks and EPID dosimetry

Chapter 8: External validation of a hidden Markov model for gamma-based classification of anatomical changes in lung cancer patients

\section{Discussion}

Chapter 9: Discussion and future perspectives

Figure 1.7: Outline of the thesis. 


\section{References}

1. KWF Kankerbestrijding. Thema Kanker. 2019; Available from: https://www.kwf.nl/kanker/ pages/default.aspx.

2. Bray F, Ferlay J, Soerjomataram I, Siegel RL, Torre LA, and Jemal A. Global cancer statistics 2018: GLOBOCAN estimates of incidence and mortality worldwide for 36 cancers in 185 countries. CA: A Cancer Journal for Clinicians, 2018. 68(6): p. 394-424.

3. International Agency for Research on Cancer (IARC). Factsheet: World. 2019; Available from: http://gco.iarc.fr/today/data/factsheets/populations/900-world-fact-sheets.pdf.

4. World Health Organization (WHO). Cancer. 2018; Available from: https://www.who.int/newsroom/fact-sheets/detail/cancer.

5. International Agency for Research on Cancer (IARC). Cancer Tomorrow. 2018; Available from: http://gco.iarc.fr/tomorrow/.

6. Yap ML, Zubizarreta E, Bray F, Ferlay J, and Barton M. Global access to radiotherapy services: Have we made progress during the past decade? Journal of Global Oncology, 2016. 2(4): p. 207-215.

7. Particle Therapy Co-Operative Group (PTCOG). Statistics of patients treated in particle therapy facilities worldwide. 2019; Available from: https://www.ptcog.ch/index.php/patientstatistics.

8. Bortfeld T. IMRT: A review and preview. Physics in Medicine and Biology, 2006. 51(13): p. R36379.

9. Otto K. Volumetric modulated arc therapy: IMRT in a single gantry arc. Medical Physics, 2008. 35(1): p. 310-7.

10. Teoh M, Clark C, Wood K, Whitaker S, and Nisbet A. Volumetric modulated arc therapy: A review of current literature and clinical use in practice. British Journal of Radiology, 2011. 84: p. 967-96.

11. The International Commission on Radiation Units and Measurements (ICRU). Prescribing, recording, and reporting photon-beam intensity-modulated radiation therapy (IMRT) (ICRU Report 83). Journal of the ICRU, 2010. 10(1).

12. Bortfeld T. Optimized planning using physical objectives and constraints. Seminars in Radiation Oncology, 1999. 9(1): p. 20-34.

13. Drzymala RE, Mohan R, Brewster L, Chu J, Goitein M, Harms W, and Urie M. Dose-volume histograms. International Journal of Radiation Oncology • Biology • Physics, 1991. 21(1): p. 71-78.

14. Herman MG. Clinical use of electronic portal imaging. Seminars in Radiation Oncology, 2005. 15(3): p. 157-167.

15. Oelfke U, Tücking T, Nill S, Seeber A, Hesse B, Huber P, and Thilmann C. Linac-integrated kVcone beam CT: Technical features and first applications. Medical Dosimetry, 2006. 31(1): p. 62-70.

16. Yan D, Vicini F, Wong J, and Martinez A. Adaptive radiation therapy. Physics in Medicine and Biology, 1997. 42(1): p. 123-32.

17. Nijsten SMJJG, van Elmpt WJ, Jacobs M, Mijnheer BJ, Dekker AL, Lambin P, and Minken AW. A global calibration model for a-Si EPIDs used for transit dosimetry. Medical Physics, 2007. 34(10): p. 3872-84.

18. van Elmpt W, Nijsten S, Petit S, Mijnheer B, Lambin P, and Dekker A. 3D in vivo dosimetry using megavoltage cone-beam CT and EPID dosimetry. International Journal of Radiation Oncology • Biology • Physics, 2009. 73(5): p. 1580-1587.

19. Podesta M, Nijsten SMJJG, Persoon LCGG, Scheib SG, Baltes C, and Verhaegen F. Time dependent pre-treatment EPID dosimetry for standard and FFF VMAT. Physics in Medicine and Biology, 2014. 59(16): p. 4749-68.

20. van Elmpt WJC, Nijsten SMJJG, Mijnheer BJ, and Minken AWH. Experimental verification of a portal dose prediction model. Medical Physics, 2005. 32(9): p. 2805-18. 
21. van Elmpt W, Nijsten SMJJG, Schiffeleers RFH, Dekker ALAJ, Mijnheer BJ, Lambin P, and Minken AWH. A Monte Carlo based three-dimensional dose reconstruction method derived from portal dose images. Medical Physics, 2006. 33(7): p. 2426-34.

22. Low DA, Harms WB, Mutic $S$, and Purdy JA. A technique for the quantitative evaluation of dose distributions. Medical Physics, 1998. 25(5): p. 656-61.

23. Low DA and Dempsey JF. Evaluation of the gamma dose distribution comparison method. Medical Physics, 2003. 30(9): p. 2455-64.

24. Podesta M, Persoon LCGG, and Verhaegen F. A novel time dependent gamma evaluation function for dynamic 2D and 3D dose distributions. Physics in Medicine and Biology, 2014. 59(20): p. 5973-85.

25. van Elmpt W, McDermott L, Nijsten S, Wendling M, Lambin P, and Mijnheer B. A literature review of electronic portal imaging for radiotherapy dosimetry. Radiotherapy and Oncology, 2008. 88(3): p. 289-309.

26. Mijnheer B. EPIDs and QA of advanced treatments. Journal of Physics: Conference Series, 2019. 1305: p. 012061.

27. Woodruff HC, Fuangrod T, Van Uytven E, McCurdy BMC, van Beek T, Bhatia S, and Greer PB. First experience with real-time EPID-based delivery verification during IMRT and VMAT sessions. International Journal of Radiation Oncology • Biology • Physics, 2015. 93(3): p. 516522.

28. Spreeuw H, Rozendaal R, Olaciregui-Ruiz I, Gonzalez P, Mans A, Mijnheer B, and van Herk M. Online 3D EPID-based dose verification: Proof of concept. Medical Physics, 2016. 43(7): p. 3969-74.

29. Working Group 1 of the Joint Committee for Guides in Metrology (JCGM/WG 1). Evaluation of measurement data - Guide to the expression of uncertainty in measurement, 2008. JCGM 100:2008

30. Nelms BE, Zhen $\mathrm{H}$, and Tomé WA. Per-beam, planar IMRT QA passing rates do not predict clinically relevant patient dose errors. Medical Physics, 2011. 38(2): p. 1037-1044.

31. Waghorn BJ, Meeks SL, and Langen KM. Analyzing the impact of intrafraction motion: Correlation of different dose metrics with changes in target D95\%. Medical Physics, 2011. 38(8): p. 4505-11.

32. Persoon LCGG, Podesta M, Hoffmann L, Sanizadeh A, Schyns LEJR, de Ruiter BM, Nijsten SMJJG, Muren LP, Troost EGC, and Verhaegen F. Is integrated transit planar portal dosimetry able to detect geometric changes in lung cancer patients treated with volumetric modulated arc therapy? Acta Oncologica, 2015. 54(9): p. 1501-07.

33. Thompson RF, Valdes G, Fuller CD, Carpenter CM, Morin O, Aneja S, Lindsay WD, Aerts HJ, Agrimson $B$, and Deville Jr C. Artificial intelligence in radiation oncology: A specialty-wide disruptive transformation? Radiotherapy and Oncology, 2018. 129(3): p. 421-426.

34. Bishop CM. Pattern recognition and machine learning. 2006, New York, NY, USA: Springer.

35. LeCun Y, Bengio Y, and Hinton G. Deep learning. Nature, 2015. 521: p. 436. 


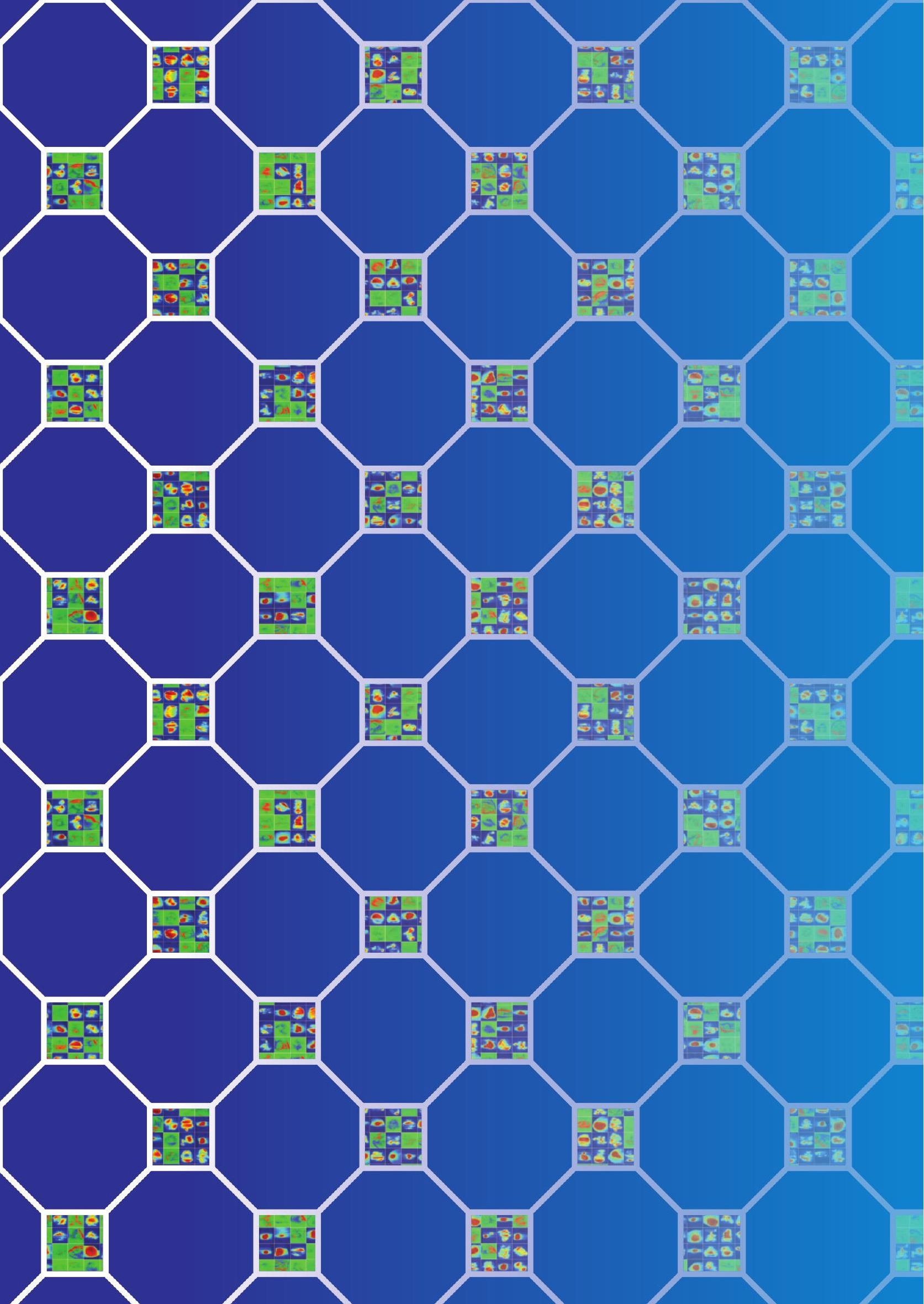




\section{Uncertainty and sensitivity of} portal dosimetry methods 



\section{Chapter 2}

\section{Validation and uncertainty analysis of a pre-treatment 2D dose prediction model}

Authors

Jose A. Baeza*, Cecile J.A. Wolfs*, Sebastiaan M.J.J.G. Nijsten ${ }^{\dagger}$ and Frank Verhaegen ${ }^{\dagger}$

${ }_{,}^{*}$ These authors contributed equally

Adapted from

Physics in Medicine and Biology, 2018. 63(3): 035033 


\begin{abstract}
Independent verification of complex treatment delivery with megavolt photon beam radiotherapy (RT) has been effectively used to detect and prevent errors. This work presents the validation and uncertainty analysis of a model that predicts 2D portal dose images (PDIs) without a patient or phantom in the beam.

The prediction model is based on an exponential point dose model with separable primary and secondary photon fluence components. The model includes a scatter kernel, off-axis ratio map, transmission values and penumbra kernels for beamdelimiting components. These parameters were derived through a model fitting procedure supplied with point dose and dose profile measurements of radiation fields. The model was validated against a treatment planning system (TPS; Eclipse) and radiochromic film measurements for complex clinical scenarios, including volumetric modulated arc therapy (VMAT). Confidence limits on fitted model parameters were calculated based on simulated measurements. A sensitivity analysis was performed to evaluate the effect of the parameter uncertainties on the model output. For the maximum uncertainty, the maximum deviating measurement sets were propagated through the fitting procedure and the model. The overall uncertainty was assessed using all simulated measurements.

The validation of the prediction model against the TPS and the film showed a good agreement, with on average $90.8 \%$ and $90.5 \%$ of pixels passing a $(2 \%, 2 \mathrm{~mm})$ global gamma analysis respectively, with a low dose threshold of $10 \%$. The maximum and overall uncertainty of the model is dependent on the type of clinical plan used as input. The results can be used to study the robustness of the model.

A model for predicting accurate 2D pre-treatment PDIs in complex RT scenarios can be used clinically and its uncertainties can be taken into account.
\end{abstract}




\section{Introduction}

The trend towards complex photon beam delivery techniques in radiotherapy, combined with hypofractionation, intensifies the need for quality assurance methods. Pre-treatment dose verification is well established in clinical practice and several methods exist to verify a treatment prior to irradiating the patient. A differentiation can be made between the detection of delivery errors with a mechanical or data transfer origin, and the detection of dosimetric deviations introduced by commercial dose engines. While the former can be detected with simplistic set-ups and efficient dose calculations, the latter entails the use of sophisticated dose engines, more accurate than the commercial methods themselves, often at the expense of longer calculation times, such as full Monte Carlo simulations. Independent fluence models and dose engines have been designed to efficiently predict the treatment delivery in pre-treatment conditions ${ }^{1-5}$. Generally, the predicted dose is intended to be compared with a measurement.

This work presents a model that provides a two-dimensional (2D) absolute dose prediction in pre-treatment conditions, i.e., without a patient or phantom in the beam. This model is integrated in our electronic portal imaging device (EPID) dosimetry routine, therefore the prediction is provided at the level of the imaging panel and it is referred to as portal dose image (PDI), although the prediction is not limited to a unique plane.

The most obvious use of the predicted PDIs is for comparison with PDIs derived from EPID measurements ${ }^{6}$. However, the utility of the prediction model is not limited to pre-treatment verification, but it can also be used for the in-house monitor unit check and the in vivo dosimetry routines. It is worth noting that predicted PDIs can be obtained from the RTPLAN, but also from the trajectory logs, allowing a more accurate description of the irradiation.

The inclusion of more, and more accurate, imaging solutions during the course of treatment together with the automation of some of the most time consuming aspects in radiotherapy planning, increase the feasibility of adaptive radiotherapy (ART) approaches. However, the effective implementation of ART necessitates quantitative knowledge of the uncertainties involved in the ART process and their propagation into the final dose calculation. Quantifying these uncertainties is expected to enable the generation of effective and customized adaptation strategies. Therefore, to evaluate the presented model's applicability as a verification method, the model uncertainty and sensitivity analysis are provided.

In summary, the aim of this work is to present a 2D dose prediction model as an independent method for pre-treatment verification. The model description is accompanied by its model fitting process and validation. Furthermore, uncertainty and sensitivity analyses are performed to evaluate the model's robustness.

\section{Materials and Methods}

\section{Theoretical model}

The presented prediction model is an exponential point dose model with separable primary and secondary (scatter) components, initially inspired by the work published by Baker et al. ${ }^{4}$, and Hounsell and Wilkinson ${ }^{7}$. The model includes an input fluence 
matrix that accounts for the dosimetric impact of the beam limiting components, extending the point dose method to a $2 \mathrm{D}$ dose prediction. This model has been developed in full scatter conditions, and provides the dose prediction in a plane within a homogeneous virtual phantom (Figure 2.1).

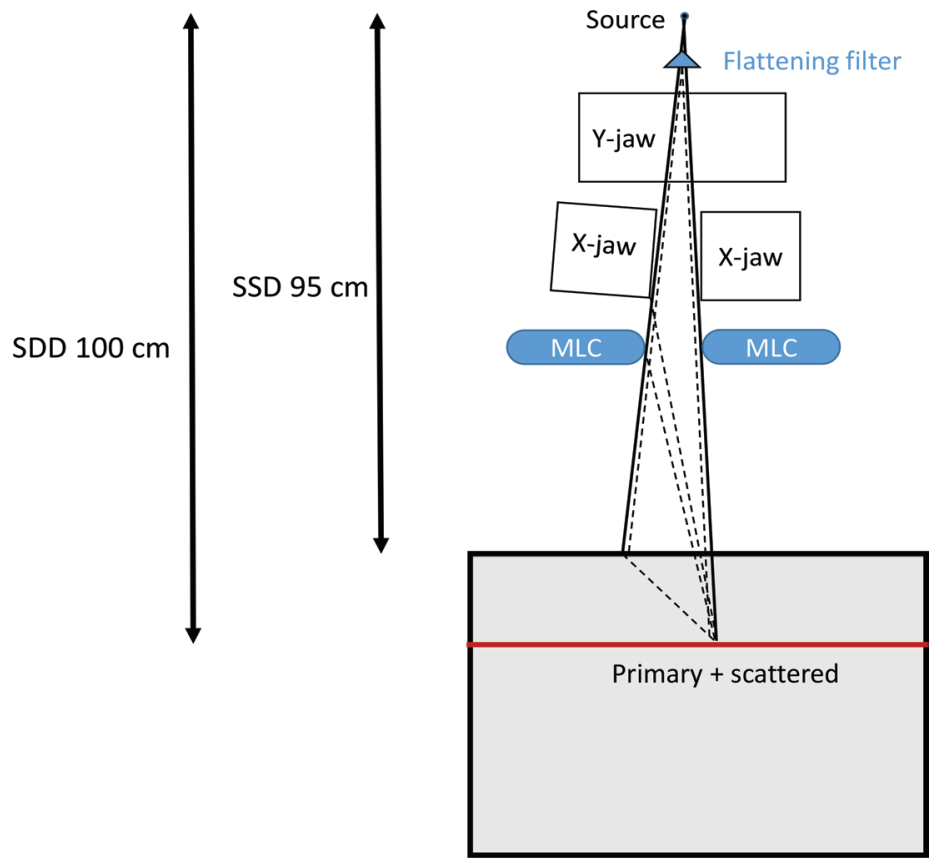

Figure 2.1: Prediction model set up showing the contribution of primary (solid lines) and scattered (dashed lines) radiation.

The model defines the dose at the beam central axis (CAX) of a circular field with radius $r$, normalized to the dose of the largest field, in this work $40 \mathrm{~cm}$, as

$$
\frac{D_{\mathrm{CAX}}(r)}{D_{\mathrm{CAX}}\left(r_{\max }\right)}=\overline{D_{\mathrm{CAX}}(r)}=1-a \cdot e^{-b \cdot r}
$$

where $a$ and $b$ are empirical parameters that establish an initial steep dose variation near the CAX, which can be attributed to the influence of the flattening filter (FF), and an extended tail with the radius $r$ that could be related to other scattering effects, as suggested by Hounsell and Wilkinson ${ }^{7}$ in a similar model. Theoretically, the model allows for the separation of primary dose $D_{p, C A X}$ and secondary dose $D_{s, C A X}$.

$$
\begin{gathered}
\overline{D_{\mathrm{p}, \mathrm{CAX}}}=1-a \\
\overline{D_{\mathrm{s}, \mathrm{CAX}}(r)}=a\left(1-e^{-b \cdot r}\right)
\end{gathered}
$$

$D_{p, C A X}$ is obtained by setting $r$ to 0 in Equation 2.1, and $D_{s, C A X}$ is obtained by subtracting $D_{p, C A X}$ from $D_{C A X}$. For an arbitrary irregular treatment field, the secondary scatter dose at 
the CAX can be obtained by integrating the individual differential contributions of $N$ circular segments of radius $r_{i}$ and area $\Delta \mathrm{A}$.

$$
\overline{D_{s, C A X}}=\frac{a \cdot b \cdot \Delta \mathrm{A}}{2 \pi} \sum_{\mathrm{i}=1}^{\mathrm{N}} \frac{\mathrm{e}^{-b \cdot r_{i}}}{r_{i}}
$$

To expand this model by Baker et al. ${ }^{4}$ from CAX to a planar prediction, a scatter kernel accounting for all scatter sources was derived from Equation 2.4 and a 2D fluence map incorporating the dosimetric characteristics of the beam delimiting components was included. The total dose can be calculated in a Cartesian coordinate system by

$$
D=D_{p}+D_{s}=\left[(1-a) \cdot \Phi(x, y)+K_{s} \otimes \Phi(x, y)\right] \cdot C
$$

where the photon fluence $\Phi$ is scaled by the primary component in the first term, and convolved with a scatter kernel $\mathrm{K}_{\mathrm{s}}$ in the second term; $\mathrm{C}$ is a normalization constant that converts monitor units to absolute dose.

The fluence is defined as

$$
\Phi(x, y)=O A R(x, y) \cdot M U \cdot \mathcal{R}(\theta) \cdot\left(S_{\mathrm{c}, \mathrm{jaws}} / S_{\mathrm{c}, \mathrm{MLC}}\right) \cdot T(x, y)
$$

where the off-axis ratio map $O A R$ is a radially symmetric primary dose distribution that includes the effects of the FF and the off-axis beam softening, MU is the number of monitor units prescribed by the treatment planning system (TPS) and the other terms are correction factors.

$\mathcal{R}$ represents a rotation by collimator angle $\theta$. The $S_{\text {c,jaws }} S_{c, M L C}$ ratio corrects the fluence for the relative difference between jaws and the MLC apertures, as the jaw shielding effect before the MLC has been demonstrated to have a non-negligible effect in dose delivery, especially for highly modulated treatments ${ }^{8}$. Finally, the transmission map $T$ is a projection of the linac head geometry at the prediction plane. $T$ is calculated using different transmission factors and penumbra kernels per beam delimiting component (X-jaws, Y-jaws, and MLC):

$$
\begin{aligned}
& T(x, y)=\left[\left\{\begin{array}{c}
1 \\
\left.T_{J_{A W}}\right|_{\forall(x, y) \in J_{A W}}
\end{array}\right\} \otimes K_{P, J A W_{x}}(x)\right] \cdot\left[\left\{\begin{array}{c}
1 \\
\left.T_{J A W_{y}}\right|_{\forall(x, y) \in J_{A W}}
\end{array}\right\} \otimes K_{P, J A W_{y}}(y)\right] . \\
& {\left[\left[\left[\left\{\begin{array}{c}
1 \\
\left.T_{\mathrm{MLC}}\right|_{\forall(\mathrm{x}, \mathrm{y}) \in \mathrm{MLC}}
\end{array}\right\} \otimes K_{\mathrm{P}, \mathrm{MLC}}(\mathrm{x})\right] \cdot\left\{\begin{array}{c}
1 \\
\left.T_{\mathrm{TG}}\right|_{\forall(\mathrm{x}, \mathrm{y}) \in \mathrm{TG}}
\end{array}\right\} \otimes K_{\mathrm{P}, \mathrm{MLC}}(\mathrm{y})\right]\right.}
\end{aligned}
$$

where $T_{\text {JAWX }}$ and $T_{\text {JAWy }}$ are transmission values for the cross-line and in-line jaw pairs respectively, and $\mathrm{K}_{\mathrm{P}, \mathrm{JAW}}$ and $\mathrm{K}_{\mathrm{P}, \mathrm{JAW}}$ are penumbra kernels for these jaws. $\mathrm{T}_{\mathrm{MLC}}$ and $\mathrm{T}_{\mathrm{TG}}$ are transmission values for the MLC, and $\mathrm{K}_{\mathrm{P}, \mathrm{MLCX}}$ and $\mathrm{K}_{\mathrm{P}, \mathrm{MLCY}}$ their penumbra kernels in the cross-line and in-line direction respectively. For every transmission factor a dedicated transmission map based on its projected geometry is created. The maps are convolved with their corresponding kernels and superimposed, resulting in an initial normalized fluence estimation.

To model the MLC leaves, two regions are differentiated: (1) an inner part, at the center of the leaf in the in-line direction, and (2) a tongue-and-groove (TG) region 
at the leaf edges. The size of these theoretical regions is defined by the TG width. To account for the rounded leaf tips a dosimetric leaf gap (DLG) width is introduced in the projected geometry of the leaf pairs that are closed.

The transmission equation (Equation 2.7) is intended for linac models with one MLC and two pairs of jaws above the MLC, such as the TrueBeam (Varian Medical Systems, Palo Alto, CA). The model can be adapted to predict dose images for other types of linacs.

\section{Model fitting}

The model fitting process comprises several independent optimization procedures in cascade, fed with point dose and dose profile measurements, as shown in Figure 2.2. Ideally, all parameters should be derived independently from a customized experiment. However, most parameters cannot be isolated. As detailed below, to address the interdependency among parameters, some of them need to be initially approximated and others require iterative optimization.

The presented model was implemented for both the TrueBeam Millennium ${ }^{\text {TM }}$ 120 Leaf MLC, and the High Definition 120 Leaf MLC (Varian Medical Systems). The TrueBeam Millennium ${ }^{\text {TM }}$ MLC, or non-STx, contains 40 inner leaf pairs of $5 \mathrm{~mm}$ width and 20 peripheral leaf pairs of $10 \mathrm{~mm}$ width, allowing a maximum static field of $40 \times 40$ $\mathrm{cm}^{2}$ at $100 \mathrm{~cm}$ from the source. The TrueBeam High Definition, or STx, MLC contains 32

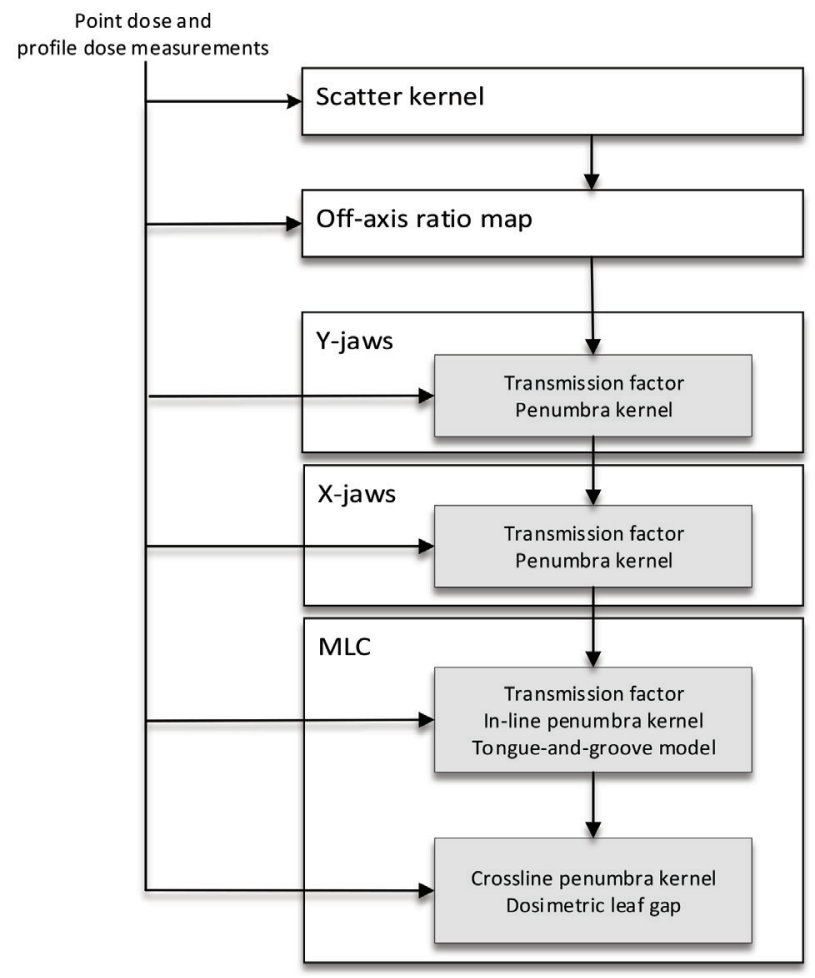

Figure 2.2: Diagram of the model fitting process. 
inner leaf pairs of $2.5 \mathrm{~mm}$ width and 28 peripheral leaf pairs of $5 \mathrm{~mm}$ width allowing a maximum static field of $40 \times 22 \mathrm{~cm}^{2}$.

The fitting process needs to be executed for every working mode, i.e., for every beam energy, fluence mode (e.g., with FF or flattening filter free (FFF)), and for different desired source-to-detector distances (SDD). For pre-treatment purposes, an SDD of $100 \mathrm{~cm}$ is ideal, but for transit dosimetry a distance of, e.g., $150 \mathrm{~cm}$ can be used.

\section{Input measurements}

To obtain point dose and dose profile measurements the prediction model's reference conditions were reproduced in the Blue Phantom 2001 water tank (Scanditronix Wellhofer, Schwarzenbruck, Germany). Measurements were obtained with a CC13 ionization chamber (Scanditronix Wellhofer) with an active volume of $0.13 \mathrm{~cm}^{3}$. No geometrical or spectral corrections were applied to the detector readout. Ideally, all model fitting inputs should have been obtained experimentally, but in this study, some of them were calculated with the TPS (Eclipse; Varian Medical Systems). The measurement set originally used for fitting is further referred to as $D_{0^{\prime}}$ and its details are outlined in Table 2.1.

Table 2.1: Overview of all fitted parameters and the measurements and calculations used as input into the fitting procedure. Measurements and calculations were performed in the model's reference conditions, i.e., SSD of $95 \mathrm{~cm}$ and SDD of $100 \mathrm{~cm}$.

\begin{tabular}{|c|c|c|c|}
\hline $\begin{array}{l}\text { Fitted } \\
\text { parameters }\end{array}$ & $\begin{array}{l}\text { Detector / } \\
\text { method }\end{array}$ & Measurements & Field size $(\mathrm{cm})$ \\
\hline$a$ and $b$ & CC13 & Point dose & $3,6,10,15,20,25,30,40$ \\
\hline \multirow[t]{2}{*}{ OAR map } & \multirow[t]{2}{*}{ CC13 } & Point dose & 10,40 \\
\hline & & Diagonal profile & 40 \\
\hline \multirow[t]{2}{*}{$\begin{array}{l}K_{P, J A W y} \\
T_{\text {JAWy }}\end{array}$} & \multirow[t]{2}{*}{$\begin{array}{l}\text { Eclipse (dose } \\
\text { calculations) }\end{array}$} & Point dose & $10,20,40$ \\
\hline & & $\begin{array}{l}\text { In-line profiles } \\
\text { (jaw-defined fields) }\end{array}$ & 10,20 \\
\hline \multirow{2}{*}{$\begin{array}{l}\mathrm{K}_{\mathrm{P}, \mathrm{JAW} x} \\
\mathrm{~T}_{\text {JAWx }}\end{array}$} & \multirow{2}{*}{$\begin{array}{l}\text { Eclipse (dose } \\
\text { calculations) }\end{array}$} & Point dose & $10,20,40$ \\
\hline & & $\begin{array}{l}\text { Cross-line profiles } \\
\text { (jaw-defined fields) }\end{array}$ & 10,20 \\
\hline \multirow{2}{*}{$\begin{array}{l}\mathrm{K}_{\mathrm{P}, \mathrm{MLCy}} \\
\mathrm{T}_{\mathrm{MLC}} \\
\mathrm{TG}_{\text {width }} \\
\mathrm{T}_{\mathrm{TG}}\end{array}$} & \multirow[t]{2}{*}{$\begin{array}{l}\text { Eclipse (dose } \\
\text { calculations) }\end{array}$} & Point dose & $\begin{array}{l}\text { 10, } 40, \text { penumbra patterns, } \\
\text { MLC transmission }\end{array}$ \\
\hline & & $\begin{array}{l}\text { In-line profiles } \\
\text { (MLC-defined fields) }\end{array}$ & $\begin{array}{l}\text { Penumbra patterns, MLC } \\
\text { transmission }\end{array}$ \\
\hline \multirow[t]{2}{*}{$\begin{array}{l}\mathrm{K}_{\mathrm{P}, \mathrm{MLCx}} \\
\text { DLG width }\end{array}$} & \multirow[t]{2}{*}{$\begin{array}{l}\text { Eclipse (dose } \\
\text { calculations) }\end{array}$} & Point dose & $\begin{array}{l}10,20,40, \text { closed leaves (DLG } \\
\text { width) }\end{array}$ \\
\hline & & $\begin{array}{l}\text { Cross-line profiles } \\
\text { (MLC-defined fields) }\end{array}$ & $\begin{array}{l}\text { 10, } 20 \text {, closed leaves (DLG } \\
\text { width) }\end{array}$ \\
\hline
\end{tabular}




\section{Scatter kernel derivation}

The first part of the model to be derived was the scatter kernel $\mathrm{K}_{s^{\prime}}$. To calculate $\mathrm{K}_{\mathrm{s}^{\prime}}$ the parameters $a$ and $b$ were fitted to output factor measurements at the CAX as in Equation 2.1. To measure the output factors, point dose measurements of square fields of different sizes were obtained (see Table 2.1). These fields were delimited by the jaws, and normalized to the largest field size.

\section{Off-axis ratio derivation}

To derive the OAR map, a rotationally symmetric map resulting from a diagonal profile of a $40 \times 40 \mathrm{~cm}^{2}$ field measured with a CC13 was used. The OAR corrects for the primary dose as in Equation 2.6 and it is independent of the scatter component as in Equation 2.5 , therefore an iterative process was used to adjust the OAR in every iteration using $\mathrm{K}_{\mathrm{s}}$ to recalculate the dose map and to compare it with the diagonal profile, until convergence. Transmission factors and penumbra kernels were set to zero.

\section{Jaw modelling}

The dosimetric impact of every jaw pair was modelled individually to fit dose profile measurements of fields delimited with the corresponding jaw pair. To optimize the transmission factor and the penumbra kernel together, the simplex search method described by Lagarias et al. ${ }^{9}$ and built-in MATLAB 2010 (MathWorks Inc., Natick, MA, USA) was used. The optimization process aimed to minimize the number of pixels failing a $2 \%$ dose difference, $2 \mathrm{~mm}$ distance to agreement, global gamma evaluation ${ }^{10}$ between the predicted and measured dose profiles.

\section{MLC modelling}

As presented in Equation 2.7, two sets of parameters were independently used to model the MLC in the in-line and cross-line directions. The in-line profile part of the model needed the determination of the transmission factors of the inner and TG region of the leaves ( $\mathrm{T}_{\text {MLC }}$ and $\mathrm{T}_{\mathrm{TG}^{\prime}}$, respectively), the $\mathrm{TG}$ region width, and the in-line penumbra kernel for the leaf $\left.\mathrm{K}_{\mathrm{P}, \mathrm{MLC} \text {. }}\right)$. To determine all parameters simultaneously the complexity of input measurements needed to be increased as shown in Figure 2.3. The cross-line MLC leaf penumbra kernel $\mathrm{K}_{\mathrm{PMLC}}$ ) was determined using simple cross-line profiles delimited with the MLC. The optimization method used to determine the MLC-related parameters consisted again of minimizing the pixels failing a gamma evaluation using MATLAB's nonlinear programming solver described in the previous section.

\section{Model validation}

The prediction model was validated against the Eclipse TPS and radiochromic EBT3 film (Ashland Inc., Lexington, USA) for treatment fields in FF mode used to treat three patients. It should be noted that the validation with the TPS is limited as some of the data used to fit the model was calculated with the TPS. The verified fields were 1) two static fields of a hybrid lung plan, 2) two arcs of a prostate plan, and 3) two arcs of a head-and-neck (H\&N) plan. Details of these treatment fields are provided in Table 2.2. To validate the model, verification plans were created setting the gantry angle to 0 
degrees for all control points.

\section{Validation against dosimetric film}

Absolute dosimetry was performed with radiochromic EBT3 films. A customized protocol adapted from Lewis et al. ${ }^{11}$ and Mathot et al. ${ }^{12}$, was followed. Two film pieces, one non-irradiated and another irradiated in absolute dosimetry reference conditions, were used to scale the calibration curve for every measurement. Films were placed at $100 \mathrm{~cm}$ from the source in a phantom consisting of $40 \times 40 \mathrm{~cm}^{2}$ slabs of solid water RW3, with a nominal density of $1.045 \mathrm{~g} \cdot \mathrm{cm}^{-3}$ (PTW, Freiburg, Germany). The SSD was set to $95 \mathrm{~cm}$ and $15 \mathrm{~cm}$ of solid water was added underneath the film.

The films were scanned using an Epson 11000XL document scanner (Shinjuku, Tokyo, Japan) in transparency mode at a spatial resolution of $50 \mathrm{dpi}$. A $4 \mathrm{~mm}$ glass plate was placed between the film and the lid of the scanner. The software FilmQA Pro 5.0 (Ashland Inc.) was used to optimize the film positioning and to convert grayscale images into absolute dose values using its triple-channel correction to diminish scanner and film artefacts ${ }^{13}$. An in-house method to correct for the non-uniform lateral response of the scanner based on the work by Lewis and Chan ${ }^{14}$ was applied.
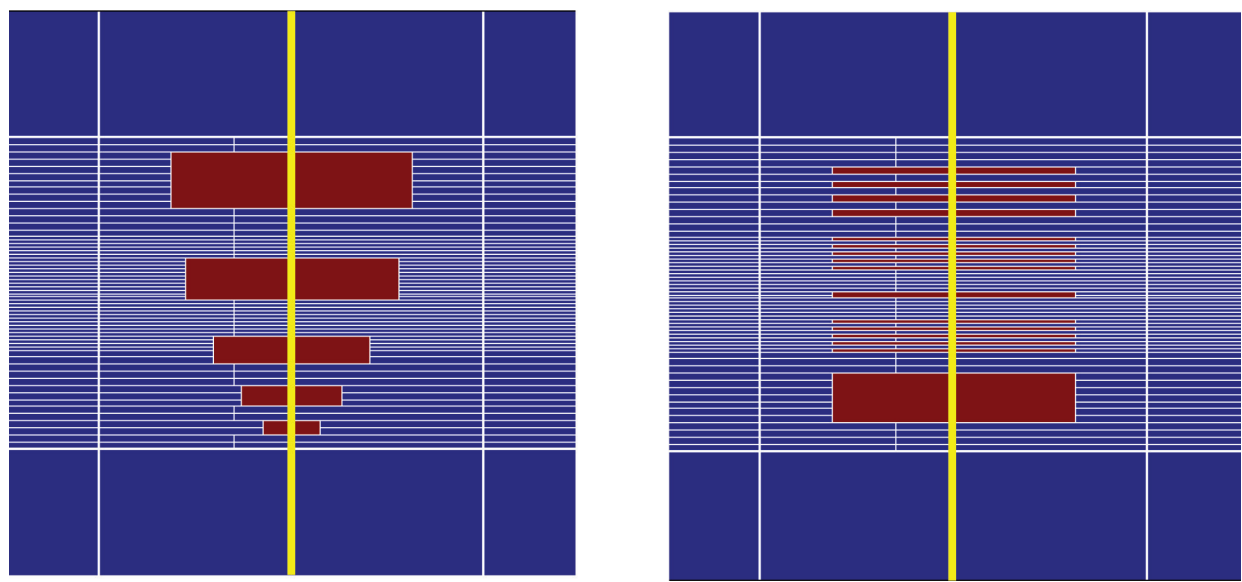

Figure 2.3: In-line penumbra patterns used for fitting $\mathrm{K}_{\mathrm{P}, \mathrm{MLC} \mathrm{y}^{\prime}} \mathrm{T}_{\mathrm{MLC}} \mathrm{T}_{\mathrm{TG}}$ and $\mathrm{TG}$ width.

Table 2.2: Details of the fields used to validate the model (AP = anterior-posterior, $\mathrm{PA}=$ posterior-anterior, $\mathrm{CW}$ $=$ clockwise, $\mathrm{CCW}=$ counter-clockwise, $\mathrm{STx}=$ TrueBeam High Definition, non-STx $=$ TrueBeam Millennium ${ }^{\mathrm{TM}}$ )

\begin{tabular}{llllll}
\hline \hline Case & Field & Type & Energy (MV) & MLC model & Monitor units \\
\hline \hline Lung & AP, PA & Static & 10 & STx & $102.7,110.8$ \\
\hline Prostate & CW, CCW & Dynamic (VMAT) & 10 & Non-STx & $123.7,126.3$ \\
\hline H\&N & CW, CCW & Dynamic (VMAT) & 6 & STx & $287.1,304.4$ \\
\hline
\end{tabular}




\section{Validation against the TPS}

To validate the prediction model against the TPS, verification plans were created in Eclipse for the selected fields. The plans were calculated with Acuros XB 11.0.31 as dose engine (Varian Medical Systems), setting the grid to $1 \mathrm{~mm}$, on a virtual $40 \times 40 \mathrm{x}$ $40 \mathrm{~cm}^{3}$ homogeneous phantom with Hounsfield units equal to zero (mass density 1.01 $\mathrm{g} \cdot \mathrm{cm}^{-3}$ and relative electron density 1.012). The plane at $5 \mathrm{~cm}$ depth was exported and compared with the model prediction.

\section{Dose comparison}

The 2D predicted dose was compared with both TPS and film measurement for the six fields, using an in-house developed gamma evaluation method ${ }^{15,16}$. Different global dose differences and distance-to-agreement criteria were used, i.e., (1\%, $1 \mathrm{~mm})$, $(2 \%, 2 \mathrm{~mm})$ and $(3 \%, 3 \mathrm{~mm})$. Film and TPS dose matrices were considered as reference to evaluate the gamma function. The results of the gamma analysis were expressed as gamma pass rates (i.e., the fraction of pixels with $|\gamma|>1$ ), which were calculated for pixels with doses above $10 \%$ of the maximum dose. Dose profiles were also obtained.

\section{Uncertainty analysis}

\section{Uncertainty of fitted parameters}

The measurement set $D_{0}$ that is used for fitting the model parameters is, due to measurement uncertainty, only one potential input into the fitting procedure. From $D_{0^{\prime}}$ the fitted parameter set $p_{0}$ is derived, which is in turn only one potential representation of the true (unknown) parameter set $p_{\text {true }}$. This means that, had the measurements differed slightly and resulted in measurement set $D_{x^{\prime}}$ a different parameter set $p_{x}$ would have been derived, which would also be only one potential representation of $\mathrm{p}_{\text {true }}$. Thus, there exists a distribution of possible parameter sets, meaning that each fitted parameter has its own uncertainty, which can be determined by establishing their confidence limits ${ }^{17}$.

Confidence limits for the fitted parameters were established by simulating other possible measurement sets $D_{j}(j=1, \ldots, n)$, which were within the measurement uncertainty of $D_{0}$. The model was then fitted using $D_{j}$ as input, leading to the distribution of possible parameter sets $p_{j}$. The simulations of $D_{j}$ were based on $D_{0}$ and its uncertainties. An overview of all measurements used as input for the fitting procedure is provided in Table 2.1. The uncertainty of each of the inputs was considered to be $1 \%$, and this value was taken as one standard deviation in a normal distribution, instead of as the maximum uncertainty ${ }^{18}$. The analysis can similarly be repeated for any level of uncertainty.

The simulated measurements $D_{j}$ were randomly sampled from a normal distribution, with the mean being the actual measurements $D_{0}$ and the standard deviation being the uncertainty of those measurements. Each point dose measurement was sampled independently, while the profile measurements were sampled as a whole, i.e., they were scaled by a random factor which was dependent on the measurement uncertainty. For each original measurement, 100 measurements were simulated $(n=$ 100). 
From the distribution $\left(p_{j}-p_{0}\right)$, the confidence limits of the fitted parameters were established. The $68 \%, 90 \%$ and $95 \%$ lower and upper confidence limits were determined by calculating the $16^{\text {th }}$ and $84^{\text {th }}, 5^{\text {th }}$ and $95^{\text {th }}$, and $2.5^{\text {th }}$ and $97.5^{\text {th }}$ percentiles, respectively. For the 2D OAR map, this was done for each pixel separately.

\section{Sensitivity analysis}

A sensitivity analysis was performed to quantify the effect of the uncertainties of the fitted parameters on the output of the prediction model. The model was used to predict PDIs for the same clinical plans used for validation, while one fitted parameter of the prediction model was changed at a time. Each parameter was changed by its $68 \%$, $90 \%$, and $95 \%$ lower and upper confidence limits, and a new PDI was predicted after each change. The original $P D I_{0}$ was compared with the $P D I_{p}$ resulting from running the model and changing a parameter. This comparison was made by calculating $\left(\mathrm{PDI}_{\mathrm{p}}-\right.$ $\left.\mathrm{PDI}_{0}\right) / \max \left(\mathrm{PDI}_{0}\right) \cdot 100$, resulting in a dose difference for each pixel as percentage of the maximum dose in $\mathrm{PDI}_{0}$. Additionally, all $\mathrm{PDI}_{\mathrm{p}}$ were compared with $\mathrm{PDI}_{0}$ by a $(2 \%, 2 \mathrm{~mm})$ gamma analysis.

To evaluate the effect of the parameter changes in different areas of the PDIs, the dose differences and gamma analysis results were analyzed in several regions of the PDI. For static fields, the in-field region, the penumbra region and the out-of-field region were used. These regions were defined in $\mathrm{PDI}_{0}$ as pixels with a value $>80 \%$ of the maximum dose, pixels with a value between $20 \%$ and $80 \%$ of the maximum dose, and pixels with a value $<20 \%$ of the maximum dose, respectively. For the dynamic fields only in-field and out-of-field regions were considered, as there is no clear penumbra region for integrated dynamic fields. In these cases, in-field pixels had a value $>50 \%$ of the maximum dose and out-of-field pixels had a value $<50 \%$ of the maximum dose.

\section{Uncertainty propagation}

After establishing the uncertainty and sensitivity for each fitted parameter, the maximum uncertainty of the model was evaluated for each of the clinical cases presented. To this end, the fitting procedure was performed, with as input the $D_{j}$ that deviated the most from $D_{0}\left(D_{\text {dev }}\right)$. The resulting parameter set $p_{j}$ was then used to predict a $P D I$ that contained the maximum uncertainty with respect to $D_{j} ; P D I\left(D_{\text {dev }}\right)$. $D_{\text {dev }}$ was determined by calculating the root mean squared error (RMSE) for each $D_{j}$ relative to $D_{0^{\prime}}$ specifically for the measurements for fitting the scatter kernel (resulting in $a$ and $b$ ) and the $O A R$. $\mathrm{PDI}\left(\mathrm{D}_{\text {dev }}\right)$ was compared with $\mathrm{PDI}_{0}$ by calculating dose deviations as in the sensitivity analysis $\left(\left(\mathrm{PDI}\left(\mathrm{D}_{\mathrm{dev}}\right)-\mathrm{PDI} \mathrm{o}_{0}\right) / \max \left(\mathrm{PDI} \mathrm{I}_{0}\right) \cdot 100\right)$, and by gamma analysis.

\section{Overall uncertainty}

Last, the overall uncertainty was calculated. This was done by using all $100 \mathrm{p}_{\text {t }}$ to predict PDIs, resulting in 100 PDIs per treatment field. These were all compared with $\mathrm{PDI}_{0^{\prime}}$ by calculating dose deviations as outlined for the sensitivity analysis and uncertainty propagation. For each of the $100 \mathrm{PDIs}$, the median value of the dose deviations in each PDI region was determined. The overall uncertainty was taken as the mean of these 100 median dose deviations. 


\section{Results}

\section{Model fitting}

The values obtained from the model fitting process for the STx MLC for the two energies used to validate the model are presented in Table 2.3.

\section{Model validation}

Figure 2.4 shows in-line and cross-line dose profiles for the three dose modalities (prediction, TPS and film) and one field from each clinical case. In all cases, the prediction model slightly overestimates the dose compared with the TPS and film, particularly in the high dose regions and for the H\&N case.

Table 2.4 summarizes the results of the gamma analyses for the model validation against film and the TPS. Gamma pass rates for $(2 \%, 2 \mathrm{~mm})$ and $(3 \%, 3 \mathrm{~mm})$ criteria show good agreement for all fields. Pass rates for $\mathrm{H} \& \mathrm{~N}$ fields are lower than for prostate and lung fields.

Figure 2.5 shows the $(2 \%, 2 \mathrm{~mm})$ gamma evaluation maps for a prostate field, which seems to indicate overestimation of the high dose regions by the prediction model when compared with the TPS, which confirms the results seen in the dose profiles.

Table 2.3: Model fitting results and 95\% confidence intervals for $6 \mathrm{MV}$ and $10 \mathrm{MV}$ beam energies, STx MLC, FF fluence mode, and $100 \mathrm{~cm}$ SDD.

\begin{tabular}{|c|c|c|}
\hline $\begin{array}{l}\text { Fitted } \\
\text { parameters }\end{array}$ & $6 \mathrm{MV}$ & $10 \mathrm{MV}$ \\
\hline$a$ & $0.2883[0.2516-0.3788]$ & $0.2466[0.2066-0.3531]$ \\
\hline$b\left(\mathrm{~cm}^{-1}\right)$ & $0.1607[0.1238-0.2402]$ & $0.1827[0.1347-0.3097]$ \\
\hline $\mathrm{K}_{\mathrm{P}, \mathrm{JAW} y}$ & $0.3543[0.1695-0.4653]$ & $0.4307[0.2853-0.5538]$ \\
\hline $\mathrm{T}_{\text {JAWy }}$ & $\begin{array}{l}3.964 \cdot 10-8\left[2.138 \cdot 10^{-10} \text { - }\right. \\
\left.8.000 \cdot 10^{-5}\right]\end{array}$ & $\begin{array}{l}9.270 \cdot 10-9\left[2.855 \cdot 10^{-9}-\right. \\
\left.4.647 \cdot 10^{-4}\right]\end{array}$ \\
\hline $\mathrm{K}_{\mathrm{P}, \mathrm{JAWx}}$ & $0.3179[0.1413-0.3776]$ & $0.4119[0.2450-0.5233]$ \\
\hline$T_{\text {JAWx }}$ & $\begin{array}{l}3.936 \cdot 10^{-8}\left[3.389 \cdot 10^{-11}-\right. \\
\left.9.691 \cdot 10^{-5}\right]\end{array}$ & $\begin{array}{l}2.131 \cdot 10^{-7}\left[1.4351 \cdot 10^{-11} \text { - }\right. \\
\left.9.857 \cdot 10^{-4}\right]\end{array}$ \\
\hline $\mathrm{K}_{\mathrm{P}, \mathrm{MLCY}}$ & $0.2178[0.1516-0.2220]$ & $0.2268[0.2096-0.3966]$ \\
\hline $\mathrm{T}_{M L C}$ & $0.0098\left[6.227 \cdot 10^{-6}-0.0157\right]$ & $0.0124\left[4.2968 \cdot 10^{-4}-0.0187\right]$ \\
\hline TG width $(\mathrm{cm})$ & $0.0689[0.0656-0.1085]$ & $0.0735[0.0659-0.1019]$ \\
\hline $\mathrm{T}_{\mathrm{TG}}$ & $0.0892[0.0140-0.1603]$ & $0.1499[0.0225-0.2620]$ \\
\hline $\mathrm{K}_{\mathrm{P}, \mathrm{MLCX}}$ & $0.2671[0.1544-0.3914]$ & $0.4988[0.1945-0.5189]$ \\
\hline DLG width (cm) & $0.1175[0.1104-0.1748]$ & $0.1876[0.1144-0.2325]$ \\
\hline
\end{tabular}



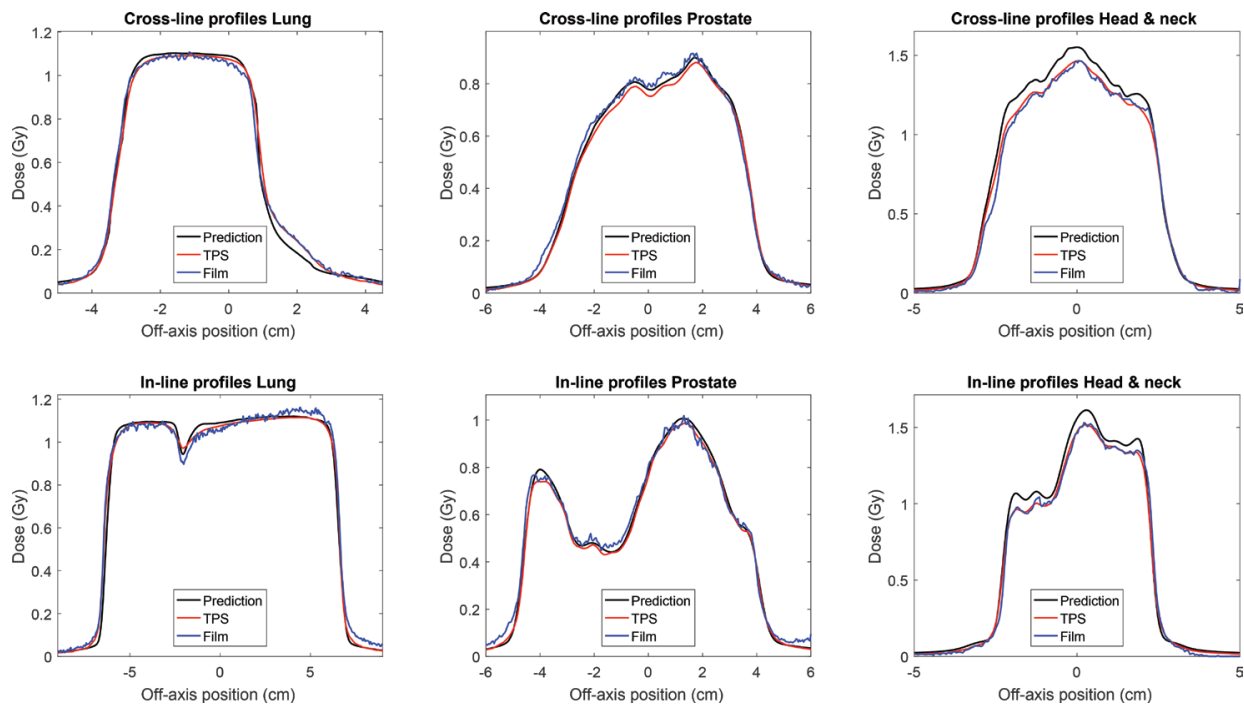

Figure 2.4: Cross-line and in-line profiles for one field of each of the clinical cases and for each method. Profiles were taken through the center of each field.

Table 2.4: Pass rates for the gamma analysis of the prediction versus the TPS and film measurement. (AP = anterior-posterior, $\mathrm{PA}=$ posterior-anterior, $\mathrm{CW}=$ clockwise, $\mathrm{CCW}=$ counter-clockwise)

\begin{tabular}{lccccccc}
\hline \hline \multicolumn{7}{c}{$(1 \%, 1 \mathrm{~mm})$} & \multicolumn{2}{c}{$(2 \%, 2 \mathrm{~mm})$} & \multicolumn{2}{c}{$(3 \%, 3 \mathrm{~mm})$} \\
\hline \hline Case & Field & TPS & Film & TPS & Film & TPS & Film \\
\hline Lung & AP & 53.3 & 45.7 & 90.9 & 81.1 & 98.7 & 92.2 \\
& PA & 54.1 & 45.5 & 92.0 & 90.0 & 99.1 & 98.1 \\
\hline \multirow{2}{*}{ Prostate } & CW & 64.8 & 64.6 & 96.7 & 99.3 & 99.8 & 100 \\
& CCW & 74.2 & 57.4 & 97.4 & 93.4 & 99.8 & 99.4 \\
\hline H\&N & CW & 34.1 & 31.9 & 80.8 & 87.3 & 92.2 & 97.1 \\
& CCW & 35.3 & 49.4 & 87.2 & 91.7 & 94.9 & 97.0 \\
\hline
\end{tabular}
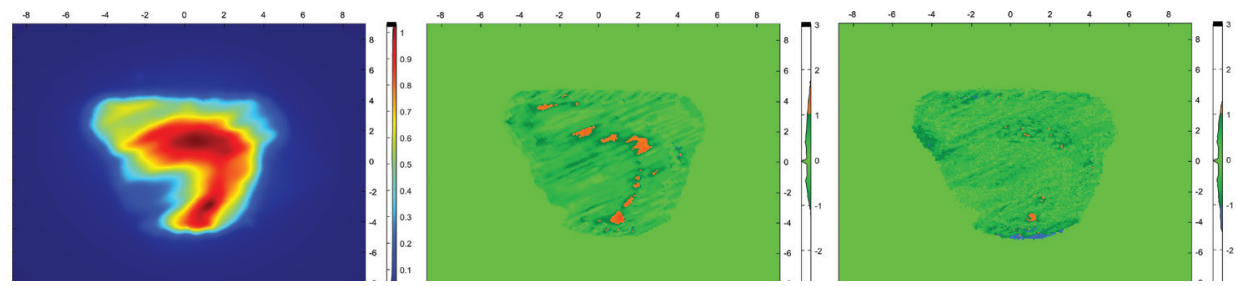

Figure 2.5: PDI (left) and (2\%, $2 \mathrm{~mm}$ ) gamma maps for one field of the prostate case, for both the validation against TPS (middle) and against film (right). The color bar of the PDI represents the absolute dose in Gy, those of the gamma maps represent the gamma values. The tick marks show the dimensions of the images in $\mathrm{cm}$. 


\section{Uncertainty analysis}

\section{Uncertainty of fitted parameters}

Figure 2.6 shows the results for the parameters $a$ and $b$ for the repeated random fitting process, whereas Table 2.3 provides the confidence limits for the fitted parameters for one accelerator type and two beam energies. The latter shows that the uncertainty is quite large for most parameters. It should be noted that the value of some of these parameters is around zero. Hence, a large change results in a value that is still very small, and will likely have little influence on the model output. What is also evident from Table 2.3, is that some parameters have a clear bias towards a positive or negative difference (e.g., $K_{P, M L C y}$ ), despite the symmetric simulated variation of $D_{j}$ around $D_{0}$. Except for a few cases, the parameters show similar trends for different accelerator types (STx vs. non-STx) and energies. The results for the other energies and accelerator types, as well as an overview of the relative differences for each parameter at all confidence limits are provided in Supplementary Material 2A.

\section{Sensitivity analysis}

With the known uncertainties of each parameter for simulated $D_{j}$, the question is how these propagate through the model and which parameters are most sensitive to changes in the dataset used for fitting. Figure 2.7 shows the results of the sensitivity analysis for one field of each of the three clinical plans, for the $95 \%$ confidence limits of some of the fitted parameters. The results for all parameters and confidence limits are provided in Supplementary Material 2B.

Each parameter was changed to its lower and upper confidence limits, leading to multiple PDI ${ }_{p}$ per changed parameter. These $\mathrm{PDI}_{\mathrm{p}}$ were compared with $\mathrm{PDI}_{0}$ by calculating for each pixel the dose deviation as percentage of the maximum dose in $\mathrm{PDI}_{0}$. From these values, multiple boxplots per PDI were created: one for each region in the PDI, i.e., in-field, penumbra and out-of-field for static fields and in-field and out-offield for dynamic fields. The boxplots thus represent the dose deviations for all pixels in a certain PDI region.
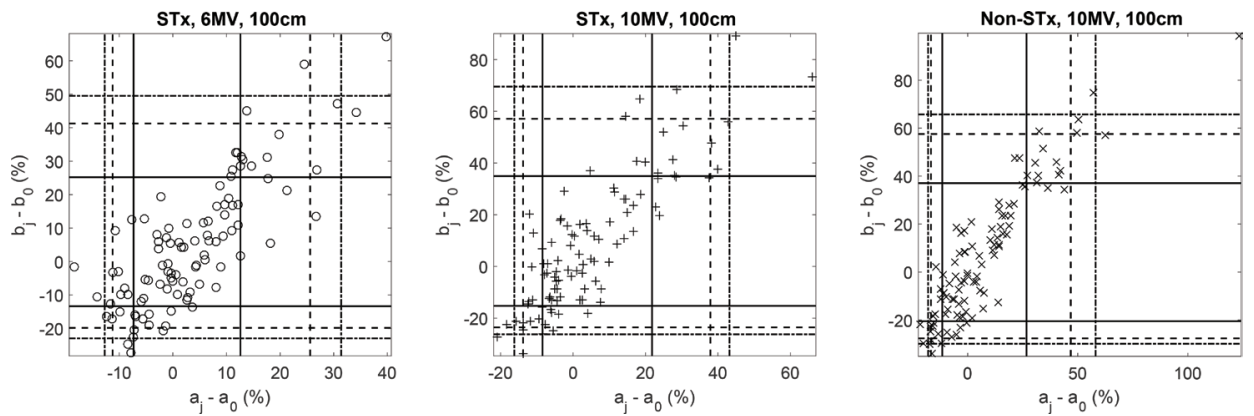

Figure 2.6: The distributions of model parameters $a$ and $b$, based on random variation of fitting the model with simulated datasets, for the accelerator types and photon beam energies of the clinical plans used for validation. The vertical and horizontal lines represent the confidence limits of $a$ and $b$, respectively. The solid lines are the $68 \%$ confidence limits, the dashed lines the $90 \%$ limits, and the dash-dotted lines the $95 \%$ limits. 

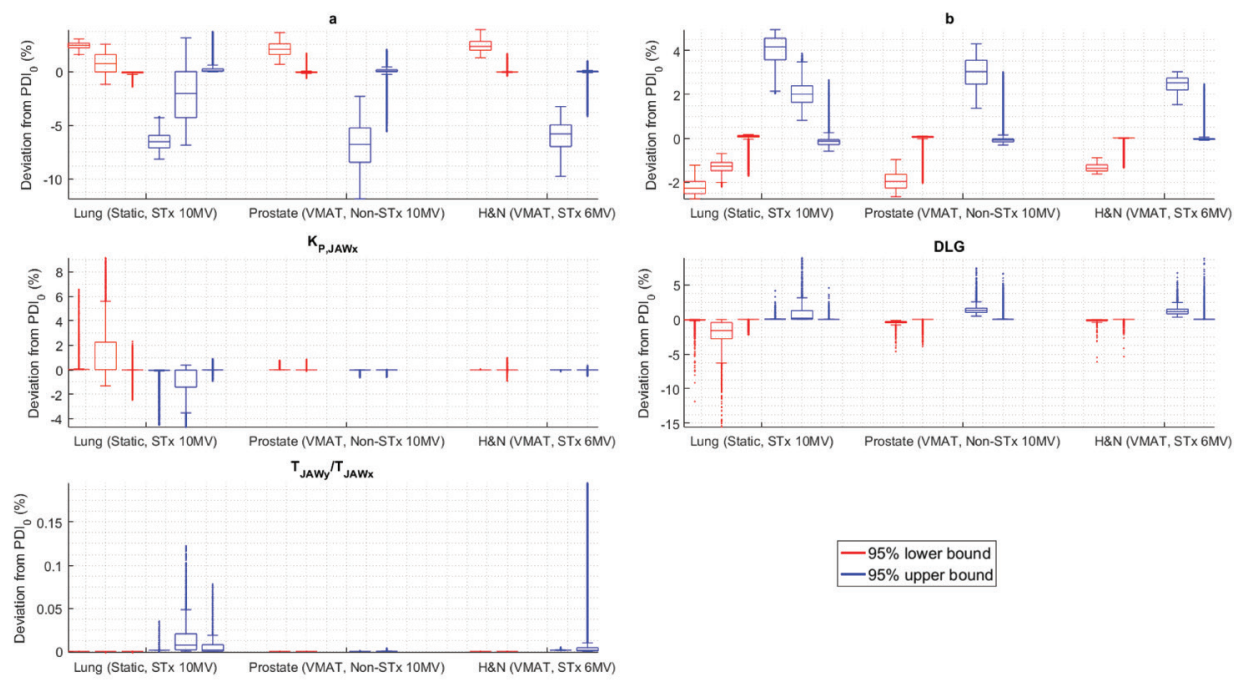

$-95 \%$ lower bound
$-95 \%$ upper bound

Figure 2.7: Boxplots for the dose deviations caused by changing each parameter separately and predicting a new PDI. The two colors represent the lower and upper $95 \%$ confidence limit to which each parameter was changed. For every color/confidence limit there are multiple boxplots, representing the different regions of the PDIs, i.e., in-field (left), penumbra (middle) and out-of-field (right) for static fields and in-field (left) and out-of-field (right) for integrated dynamic fields.
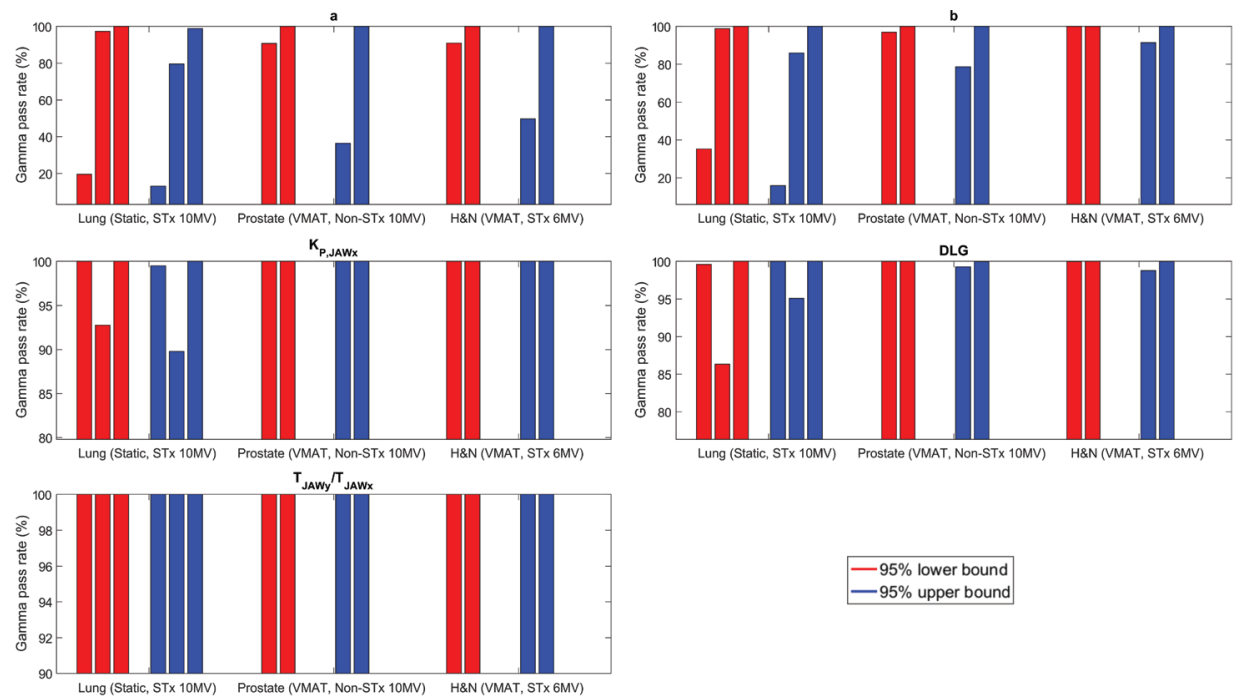

- $95 \%$ lower bound - $95 \%$ upper bound

Figure 2.8: Gamma pass rates obtained by comparing PDI with PDI with a (2\%, $2 \mathrm{~mm})$ gamma analysis. The multiple bars per color/confidence limit represent the different regions of the PDI (from left to right: in-field, penumbra and out-of-field for static fields, in-field and out-of-field for dynamic fields). 
What can be derived from these results is that for the in-field regions, $a$ and $b$ are the most sensitive parameters, i.e., their uncertainties cause the largest dose deviations. The penumbra kernels are more sensitive in the static field than in the dynamic ones. In the static field their effect is, as can be expected, most pronounced in the penumbra region. The same holds for other beam-delimiting parameters such as the DLG width. In the out-of-field region, relative to the field's maximum dose, none of the parameters is very sensitive to changes. Figure 2.7 also shows that the very large uncertainty in the jaw transmission parameters has very little influence on the resulting PDI. Together with the OAR map, whose uncertainty was also small, these seem to be the least sensitive parameters.

The results of the gamma analyses are very similar. As can be seen in Figure 2.8 , the gamma pass rates show the same pattern as the dose differences. Lower pass rates are seen for parameters $a$ and $b$ in the in-field region, and for the penumbra kernels in the penumbra region of the static field. For the transmission parameters and $\mathrm{OAR}$, all pixels pass the gamma analysis for all cases.

\section{Uncertainty propagation}

To determine the maximum uncertainty for each of the clinical plans evaluated, PDIs were predicted using the parameter set fitted with the maximum deviating measurement set $D_{\text {dev }}$. Similar to Figure 2.7, boxplots were created to visualize the dose differences (Figure 2.9). Most notable in this figure are the large deviations in the infield region of the $\mathrm{H} \& \mathrm{~N}$ case, and the large spread of deviations in the penumbra region of the lung case. The prostate case shows the least variation. The dose deviations in the out-of-field region are small for all three cases.

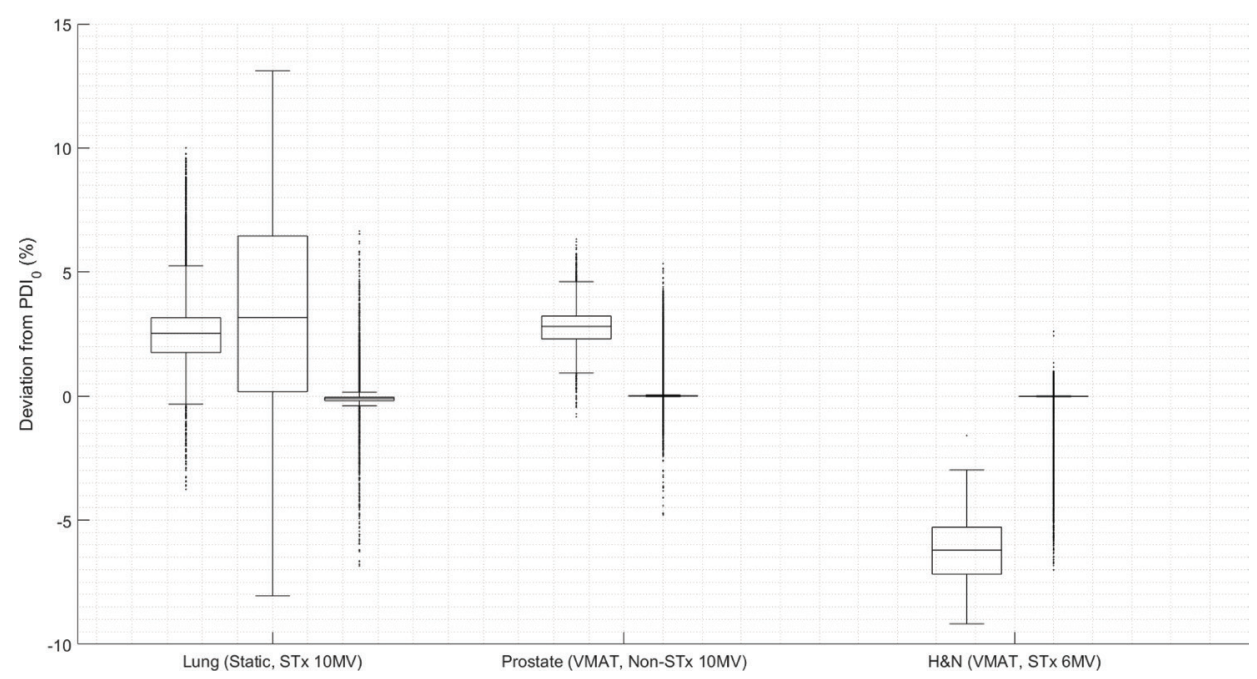

Figure 2.9: Maximum uncertainty for one field of each validated clinical plan, evaluated by predicting a PDI based on parameters fitted with $D_{\text {dev }}$. The multiple boxplots per clinical case again stand for the different regions of the PDIs, as described in Figure 2.7. 
For the prostate case, the comparison between $\mathrm{PDI}$, and $\mathrm{PDI}\left(\mathrm{D}_{\text {dev }}\right)$ by dose difference and by a $(2 \%, 2 \mathrm{~mm})$ gamma analysis are displayed in Figure 2.10. As expected from Figure 2.9, the in-field region shows the largest dose differences. When comparing with a gamma analysis, many of the differences are not visible anymore. However, the area with the largest dose deviations does not pass the gamma analysis.

\section{Overall uncertainty}

The overall uncertainty, incorporating all $100 \mathrm{p}_{\mathrm{i}}$, is shown in Table 2.5. Comparing these overall uncertainties with the maximum uncertainties, the same trends are observed. The uncertainties in the $\mathrm{H} \& \mathrm{~N}$ case and penumbra region of the lung case are largest, whereas those in the out-of-field regions are close to zero.

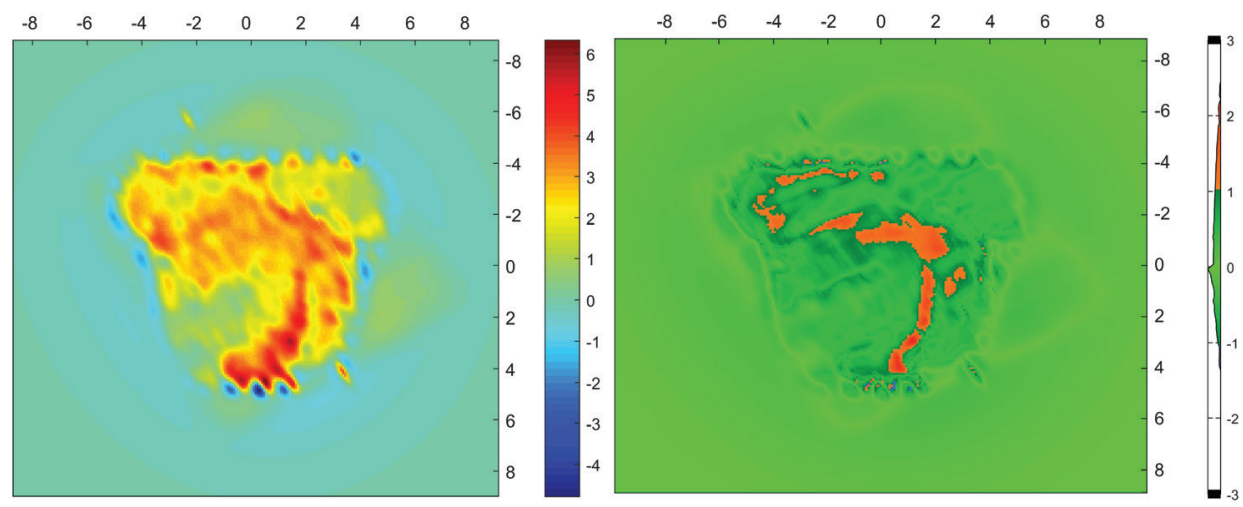

Figure 2.10: Percentage dose difference (left) and (2\%, $2 \mathrm{~mm})$ gamma analysis (right) for one field of the prostate case (VMAT, Non-STx, $10 \mathrm{MV}$ ), for the comparison of PDI( $\left.\mathrm{D}_{\text {dev }}\right)$ and PDI. The color bar of the left image represents the dose difference as percentage of the maximum dose, that of the gamma map represents the gamma values. The tick marks show the dimensions of the images in $\mathrm{cm}$.

Table 2.5: Overall uncertainty of the clinical plans.

\begin{tabular}{|c|c|c|c|c|}
\hline \multirow[b]{2}{*}{ Case } & \multirow[b]{2}{*}{ Field } & \multicolumn{3}{|c|}{ Overall uncertainty (\%) } \\
\hline & & In-field & Penumbra & Out-of-field \\
\hline \multirow[t]{2}{*}{ Lung } & AP & 0.3 & 1.5 & $-1.1 \cdot 10^{-2}$ \\
\hline & PA & 0.3 & 1.1 & $-1.1 \cdot 10^{-2}$ \\
\hline \multirow[t]{2}{*}{ Prostate } & CW & 0.6 & - & $1.1 \cdot 10^{-3}$ \\
\hline & $\mathrm{CCW}$ & 0.4 & - & $0.6 \cdot 10^{-3}$ \\
\hline \multirow[t]{2}{*}{$\mathrm{H} \& \mathrm{~N}$} & CW & -1.6 & - & $-2.9 \cdot 10^{-3}$ \\
\hline & CCW & -1.7 & - & $-2.6 \cdot 10^{-3}$ \\
\hline
\end{tabular}




\section{Discussion}

A pre-treatment dose prediction model has been presented. The physical description of the model was complemented by detailing its fitting process and its required input measurements. The model underwent an exhaustive uncertainty analysis to understand its robustness with regard to reasonable deviations of fitting data. This type of sensitivity analysis is rarely performed in radiotherapy modelling.

The results of the validation showed good overall agreement between the prediction and both TPS and radiochromic film measurements. The largest deviations were found for the H\&N case, probably due to the highly modulated nature of this case. This could indicate that the prediction model is less accurate for small fields. The role of penumbra regions and inter-leaf transmission is also expected to be more noticeable in highly modulated fields. Including small fields in the model fitting process may mitigate the overestimation of the prediction model compared with the TPS and film measurements.

Furthermore, the dose profiles seem to indicate a general overestimation when comparing the prediction model to the TPS. However, to confirm a systematic effect, the model needs to be validated for more clinical cases.

The uncertainty analysis of the fitted parameters shows that changing the measurements used for fitting can have a large effect on the values of some of these parameters. It should be noted that the measurement uncertainty used in this work might be too large. As the standard deviation of the normal distribution was considered to be $1 \%$, differences in measurement values up to $3 \%$ were possible.

However, the sensitivity analysis shows that some very large changes in parameter values have very little effect on the resulting PDI. The parameters whose effect is most notable in the output of the model are $a$ and $b$. As expected, penumbra kernels have an impact particularly in the penumbra regions. Their sensitivity is larger for static fields, which have sharper penumbras than integrated dynamic fields. Outof-field, the effect of any parameter deviation is negligible.

As many parameters show little sensitivity, it might be possible to convert some of these into constants. This would reduce the time needed for fitting, and would make the model simpler. An example of this are the transmission parameters, especially those for the jaws. Even though they have a very large uncertainty, changing their values barely has an effect on the model output.

Comparing parameter uncertainties between accelerator types, the parameters that show the most prominent differences are $\mathrm{K}_{\mathrm{P}, \mathrm{MLCY}} \mathrm{K}_{\mathrm{P}, \mathrm{MLCx^{ \prime }}} \mathrm{T}_{\mathrm{MLC}}$ and the TG and DLG parameters. Not surprisingly, all these parameters are related to the MLC geometry, which is the main difference between the two accelerator types.

Propagating the uncertainties from fitting the model with a measurement set that deviates maximally from $D_{0}$ gives very different results depending on the clinical case. Especially in the $\mathrm{H} \& \mathrm{~N}$ case, the deviation from $\mathrm{PDI}_{0}$ in the in-field region is much larger than in the other two cases. It needs to be investigated further if this is caused solely by the parameter uncertainty, or if the larger proportion of small fields in this plan also contributes to the larger dose deviations. The wide spread of dose differences in the penumbra region of the lung case is likely due to the fact that it concerns a static field with a sharp penumbra. This is more sensitive to changes than the more spreadout penumbra of the integrated VMAT fields. 
The uncertainties also propagated through in a $(2 \%, 2 \mathrm{~mm})$ gamma analysis. This shows that it is necessary to take the model's uncertainty into account when developing clinical decision models based on the outcome of a gamma comparison (e.g., between a pre-treatment prediction and an EPID measurement). Based on the results of the uncertainty analysis, it may be necessary to establish the uncertainty per clinical plan. To test this hypothesis, further research including more clinical cases is needed. It may be possible to establish trends per treatment site or type of plan, e.g., for plans with a larger proportion of small fields, the uncertainty of the model could be higher.

\section{Conclusion}

To conclude, this work presented a pre-treatment prediction model, including its validation and uncertainty analysis. The validation shows the model can be used clinically, but more testing is needed, especially for small fields. Furthermore, its uncertainties can be taken into account when developing clinical decision models.

\section{Acknowledgments}

The authors would like to thank Dr. Karen Zegers and Dr. Mark Podesta for their insight and fruitful discussion. This study was partially funded by Varian Medical Systems (project: TimePort-III). 


\section{References}

1. Jiang $\mathrm{SB}$, Boyer $\mathrm{AL}$, and $\mathrm{Ma} \mathrm{CM}$. Modeling the extrafocal radiation and monitor chamber backscatter for photon beam dose calculation. Medical Physics, 2001. 28(1): p. 55-66.

2. Fippel M, Haryanto F, Dohm O, Nusslin F, and Kriesen S. A virtual photon energy fluence model for Monte Carlo dose calculation. Medical Physics, 2003. 30(3): p. 301-11.

3. Van Esch A, Depuydt T, and Huyskens DP. The use of an aSi-based EPID for routine absolute dosimetric pre-treatment verification of dynamic IMRT fields. Radiotherapy and Oncology, 2004. 71(2): p. 223-34.

4. Baker CR, Clements R, Gately A, and Budgell GJ. A separated primary and scatter model for independent dose calculation of intensity modulated radiotherapy. Radiotherapy and Oncology, 2006. 80(3): p. 385-90.

5. Chytyk K and McCurdy BM. Comprehensive fluence model for absolute portal dose image prediction. Medical Physics, 2009. 36(4): p. 1389-98.

6. van Elmpt WJC, Nijsten SMJJG, Mijnheer BJ, and Minken AWH. Experimental verification of a portal dose prediction model. Medical Physics, 2005. 32(9): p. 2805-18.

7. Hounsell AR and Wilkinson JM. Head scatter modelling for irregular field shaping and beam intensity modulation. Physics in Medicine and Biology, 1997. 42(9): p. 1737-49.

8. Swinnen ACC, Ollers MC, Roijen E, Nijsten SM, and Verhaegen F. Influence of the jaw tracking technique on the dose calculation accuracy of small field VMAT plans. Journal of Applied Clinical Medical Physics, 2017. 18(1): p. 186-95.

9. Lagarias JC, Reeds JA, Wright MH, and Wright PE. Convergence properties of the Nelder-Mead simplex method in low dimensions. SIAM Journal on optimization, 1998. 9(1): p. 112-47.

10. Low DA, Harms WB, Mutic $S$, and Purdy JA. A technique for the quantitative evaluation of dose distributions. Medical Physics, 1998. 25(5): p. 656-61.

11. Lewis $D$, Micke $A, Y u X$, and Chan MF. An efficient protocol for radiochromic film dosimetry combining calibration and measurement in a single scan. Medical Physics, 2012. 39(10): p. 6339-50.

12. Mathot M, Sobczak S, and Hoornaert MT. Gafchromic film dosimetry: Four years experience using FilmQA Pro software and Epson flatbed scanners. Physica Medica, 2014. 30(8): p. 871-77.

13. Micke A, Lewis DF, and Yu X. Multichannel film dosimetry with nonuniformity correction. Medical Physics, 2011. 38(5): p. 2523-34.

14. Lewis $D$ and Chan MF. Correcting lateral response artifacts from flatbed scanners for radiochromic film dosimetry. Medical Physics, 2015. 42(1): p. 416-29.

15. Persoon LCGG, Podesta M, van Elmpt WJC, Nijsten SMJJG, and Verhaegen F. A fast threedimensional gamma evaluation using a GPU utilizing texture memory for on-the-fly interpolations. Medical Physics, 2011. 38(7): p. 4032-35.

16. Podesta M, Persoon LCGG, and Verhaegen F. A novel time dependent gamma evaluation function for dynamic 2D and 3D dose distributions. Physics in Medicine and Biology, 2014. 59(20): p. 5973-85.

17. Press WH, Teukolsky SA, Vetterling WT, and Flannery BP. Confidence limits on estimated model parameters, in Numerical recipes: The art of scientific computing. 2007, Cambridge University Press: Cambridge, UK. p. 807-18.

18. International Atomic Energy Agency (IAEA). Accuracy requirements and uncertainties in radiotherapy IAEA Human Health Series 2016. No. 31. 


\section{Supplementary Material Chapter 2}

\section{Supplementary Material 2A: Confidence limits for all fitted parameters}
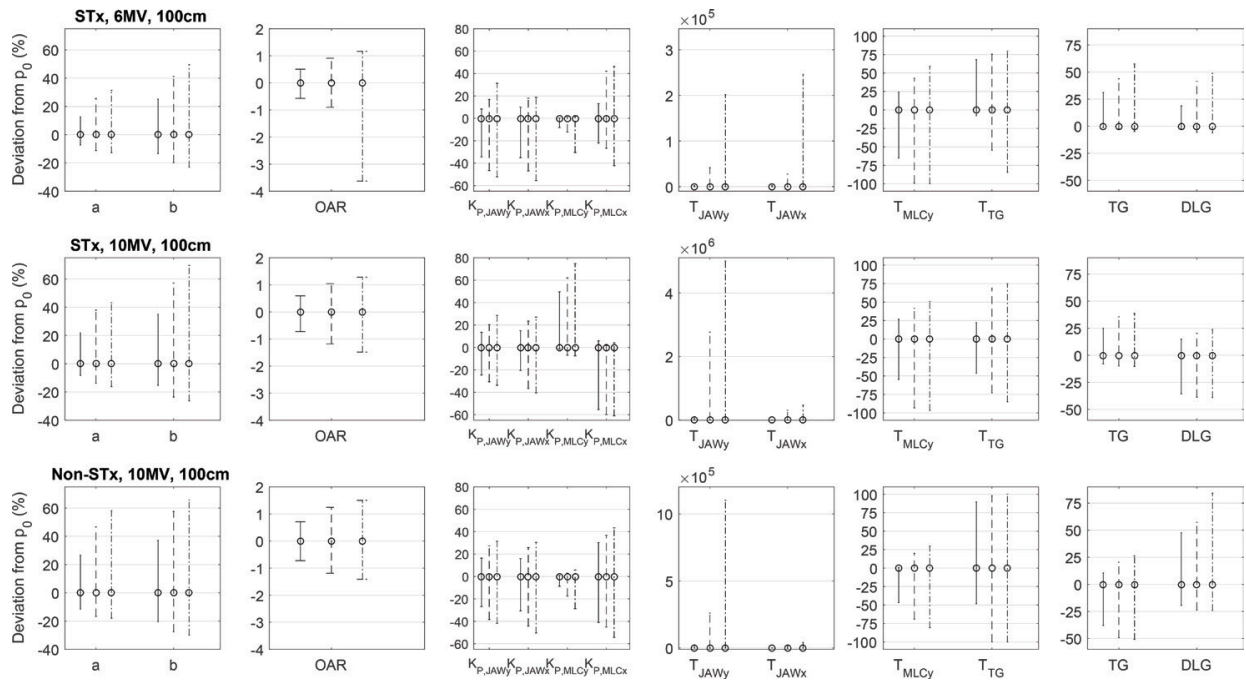

Figure 2A.1: Confidence limits for all fitted parameters, in relative terms. The solid, dashed and dash-dotted lines represent the $68 \%, 90 \%$ and $95 \%$ confidence intervals, respectively. In case of the OAR map, the mean values are displayed.
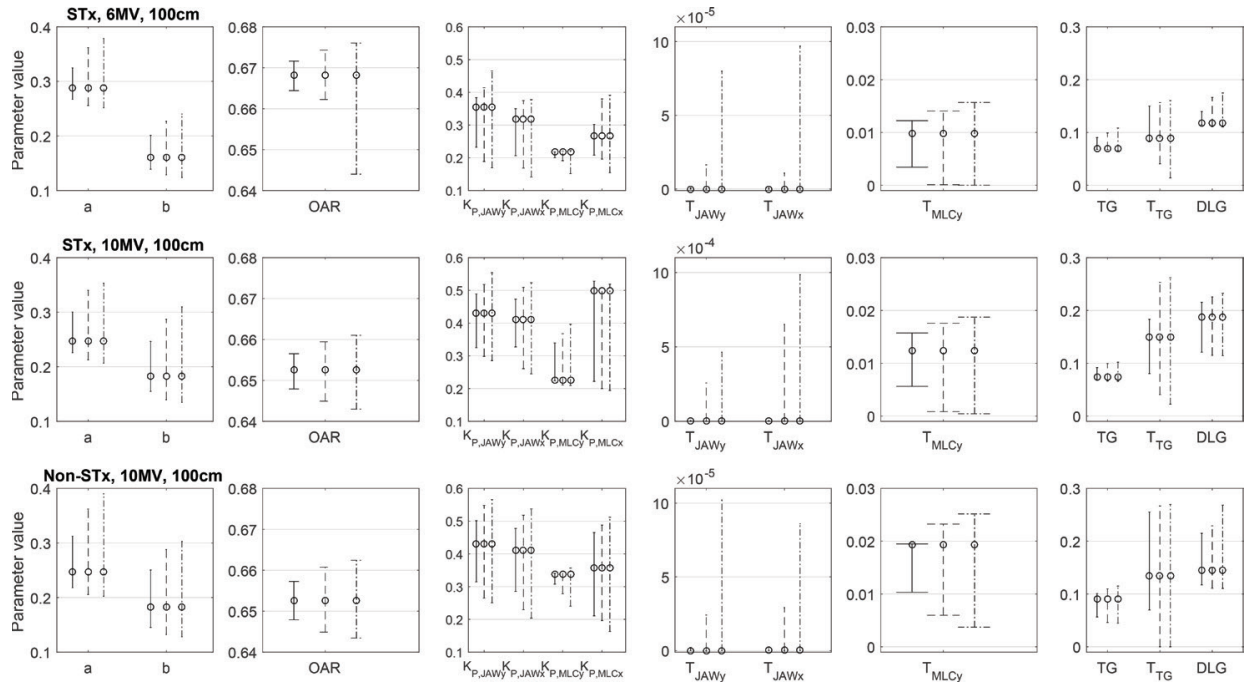

Figure 2A.2: Confidence limits for all fitted parameters, in absolute terms. The solid, dashed and dash-dotted lines represent the $68 \%, 90 \%$ and $95 \%$ confidence intervals, respectively. In case of the OAR map, the mean values are displayed. 


\section{Supplementary Material 2B: Sensitivity analysis results}

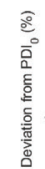
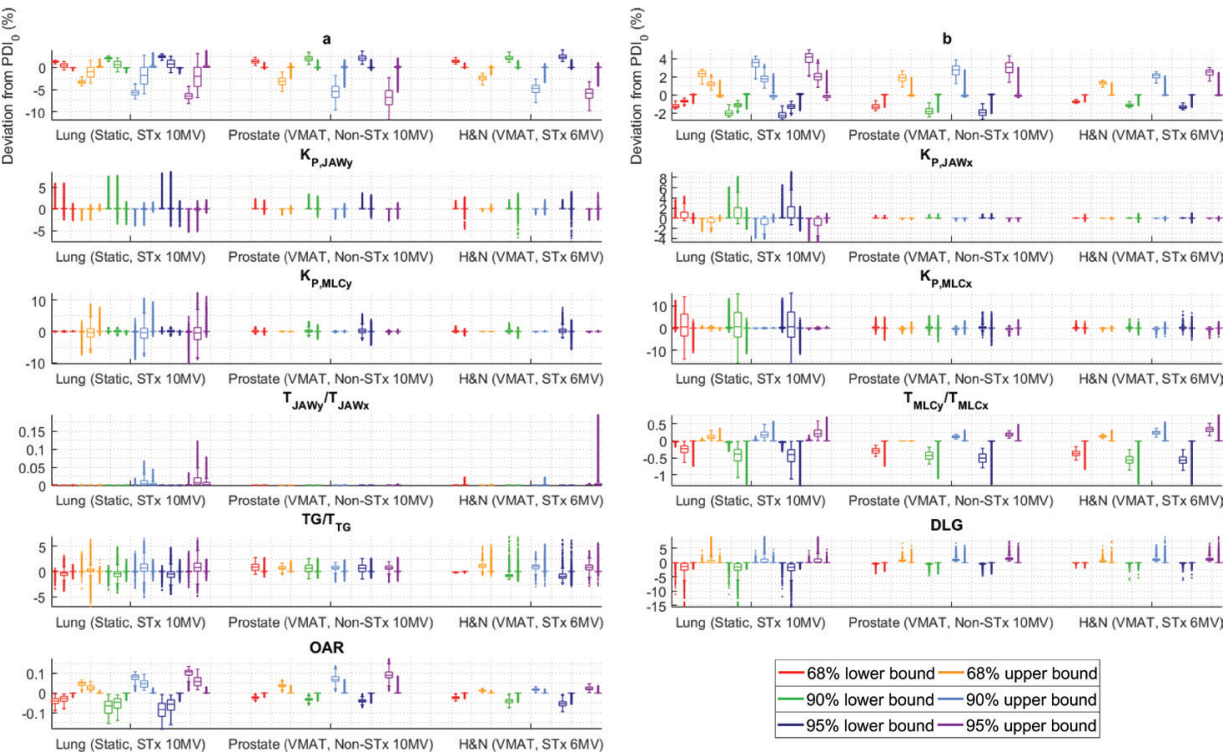

$$
\begin{array}{|l|}
\hline-68 \% \text { lower bound }-68 \% \text { upper bound } \\
\hline-90 \% \text { lower bound }-90 \% \text { upper bound } \\
-95 \% \text { lower bound }-95 \% \text { upper bound } \\
\hline
\end{array}
$$

Figure 2B.1: Sensitivity analysis results for all fitted parameters and all confidence limits.

\section{Supplementary Material 2C: Gamma analysis results of the sensitivity analysis}
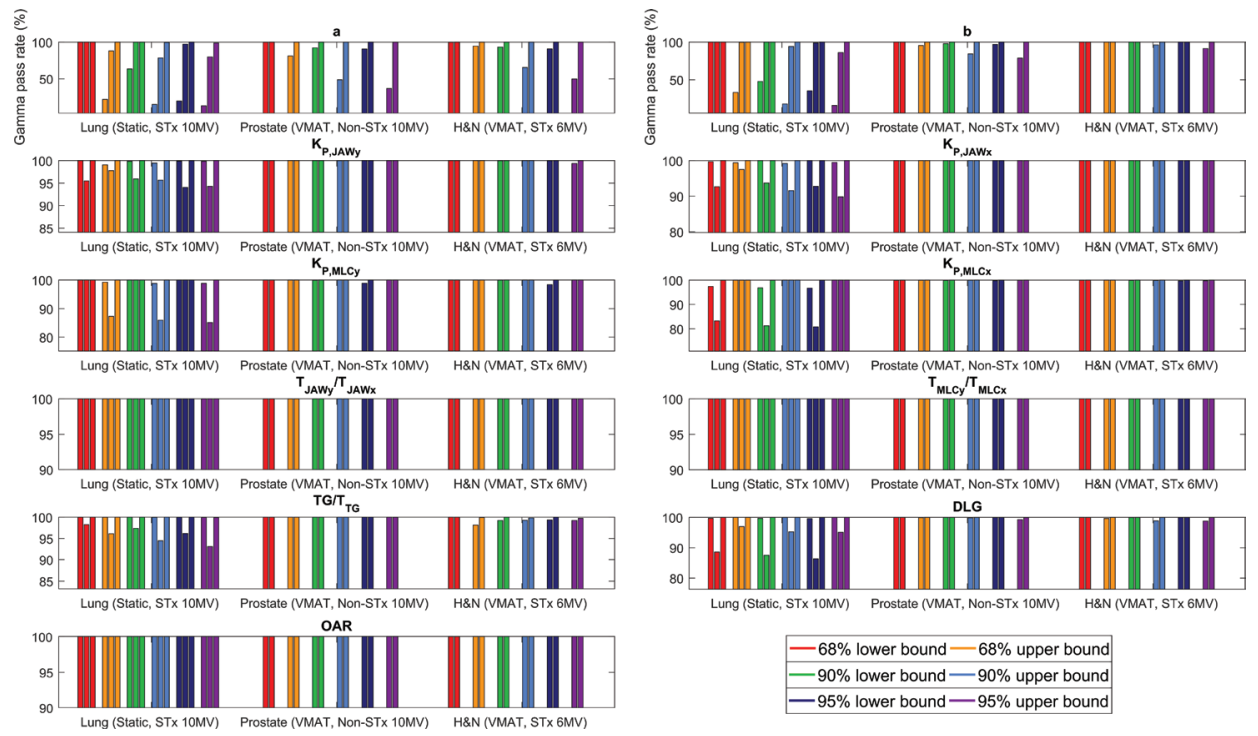

Figure 2C.1: Gamma analysis results of the sensitivity analysis for all fitted parameters and all confidence limits. 




\section{Chapter 3}

\section{Should dose from small fields be limited for dose verification procedures?: Uncertainty vs. small field dose in VMAT treatments}

Authors

Cecile J.A. Wolfs, Ans C.C. Swinnen, Sebastiaan M.J.J.G. Nijsten and Frank Verhaegen

Adapted from

Physics in Medicine and Biology, 2018. 63(20): 20NT01 


\begin{abstract}
Over the years, radiotherapy treatments have become more complex and conformal, leading to an increased use of small field segments in volumetric modulated arc therapy (VMAT) arcs. The impact of small field dose inaccuracy on dose verification methods has not been studied yet. The aim of this work is therefore to quantify the relationship between the uncertainty of a $2 \mathrm{D}$ pre-treatment dose prediction model and the proportion of dose coming from small fields in VMAT arcs for a range of clinical plans.

The model evaluated in this work predicts 2D portal dose images (PDIs) without a patient or phantom in the beam. The uncertainty of the model was calculated through simulation of model parameter deviations. The proportion of dose from small fields in a VMAT arc was determined by comparing a PDI with only dose from small fields with the original PDI. The uncertainty and proportion of dose from small fields were calculated for 109 VMAT arcs ( 41 head and neck, 33 lung, 35 prostate). The correlation was assessed with a linear regression.

There is a statistically significant positive correlation between the uncertainty of the model and the proportion of dose from small fields in a VMAT arc, for each treatment site individually, as well as for all tumor sites together. The strongest relationship is found for the prostate cases.

As there is a positive relationship between the uncertainty of the $2 \mathrm{D}$ pre-treatment dose prediction model and the proportion of dose from small fields, it may be wise to limit the dose from small fields in VMAT arcs, to avoid additional uncertainty in the dose verification process.
\end{abstract}




\section{Introduction}

Over the years, radiotherapy treatments have become more complex and conformal, going from 3D conformal radiotherapy (3DCRT) to intensity modulated radiotherapy (IMRT) and ultimately to volumetric modulated arc therapy (VMAT) ${ }^{1}$. This has increased the use of small radiation fields considerably ${ }^{2,3}$. For instance, a typical VMAT arc is highly modulated and consists of many small subfields or segments ${ }^{4}$. However, the dosimetry of small fields poses challenges, which increases the uncertainty of small field measurements and dose calculations ${ }^{3,5}$.

In contrast to dose measurements and calculations by treatment planning systems, the influence of dose from small fields on dose verification methods has not been studied yet. The uncertainty analysis of a recently introduced 2D pretreatment dose prediction model, that is used for dose verification, also showed larger uncertainties for cases with a higher fraction of dose from small fields ${ }^{6}$. When using models like these in a dose verification process, it is important to understand the behavior of these uncertainties. The purpose of this work is therefore to quantify the relationship between the uncertainty of a $2 \mathrm{D}$ pre-treatment dose prediction model and the proportion of dose coming from small fields in VMAT arcs for a range of clinical plans. This study is an addition to our previously published dose verification method ${ }^{6}$.

\section{Materials and methods}

The model evaluated in this work predicts 2D portal dose images (PDIs) without a patient or phantom in the beam, and is described in detail by Baeza et al. ${ }^{6}$ In practice, the dose prediction is then compared to an electronic portal imaging device (EPID) dose measurement, in a time-integrated fashion. Figure 3.1a shows a schematic overview of the model, its fitting process, and its input and output. The fitted parameters of the model $\left(p_{0}\right)$ are based on a measurement set $\left(D_{0}\right)$ containing point dose and profile measurements of various square and modulated fields. No fields smaller than $3 \times 3 \mathrm{~cm}^{2}$
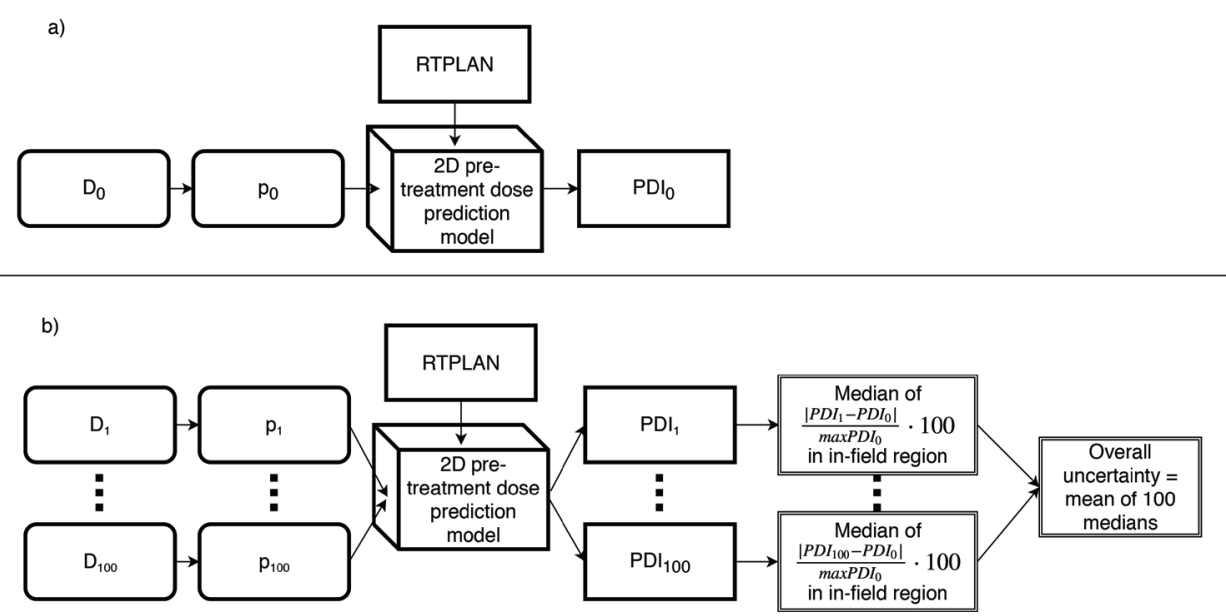

Figure 3.1: Schematic representations of a) the 2D pre-treatment prediction model and b) its uncertainty analysis. $D_{0}$ : original measurement set; $p_{0}$ : original parameter set; $P D I_{0}$ : original portal dose image; $D_{j}$ : simulated measurement set; $p_{\mathrm{i}}$ : parameter set fitted with simulated measurement set; PDI: portal dose image based on simulated data; $j=1, \ldots, 100$ 
are used for fitting the model. The $3 \times 3 \mathrm{~cm}^{2}$ field size is also used as the threshold for small fields in this work, i.e., fields below $3 \times 3 \mathrm{~cm}^{2}$ are considered small fields.

For the uncertainty analysis of the model, also presented by Baeza et al. ${ }^{6}$ and schematically shown in Figure $3.1 \mathrm{~b}$, measurement sets $\left(D_{i}\right)$ were simulated that were within the measurement uncertainty of $D_{0}$. With these simulated measurement sets, alternative parameter sets $\left(\mathrm{p}_{\mathrm{f}}\right)$ were fitted and used to predict PDIs. These PDIs were compared to the original $\mathrm{PDI}_{0}$, by calculating the median global dose deviation, pixel by pixel, in the in-field region for each PDI. The mean of these medians is the overall uncertainty (Equation 3.1). In this work, $n$ equals 100.

$$
\text { Overall uncertainty }=\frac{\sum_{j=1}^{n} \operatorname{median}\left(\frac{\left|\mathrm{PDI}_{\mathrm{j}}-\mathrm{PDI}_{0}\right|}{\mathrm{max} \mathrm{PDI}_{0}} \cdot 100\right)}{n}
$$

The proportion of dose from small fields in a VMAT arc was calculated by constructing another $\mathrm{PDI}$ from $\mathrm{PDI}_{0^{\prime}}$ that only contained the dose coming from small fields $\left(\mathrm{PDI} \mathrm{small}_{\text {fields }}\right)$. This process is illustrated in Figure 3.2. As there is no unequivocal way to calculate the equivalent field size (EFS) for a modulated field, Equation 3.2 for calculating EFS for a rectangular field ${ }^{7}$ was used.

$$
\mathrm{EFS}=\frac{2 \cdot L \cdot W}{L+W}
$$

This formula was applied to each VMAT segment of the predicted PDI. In this equation, $L$ is the length of the field, which in this work was determined by the sum of the projected MLC leaf widths at the isocenter, of the MLC leaves shaping the field. W is the width of the field, defined here as the average aperture (also projected at the isocenter) of the MLC leaves forming the field. This is depicted in Figure 3.3.

If the EFS was below or equal to $3 \times 3 \mathrm{~cm}^{2}$, the segment was considered to be a small field, and it was retained. In contrast, if the EFS exceeded $3 \times 3 \mathrm{~cm}^{2}$, the segment was deemed to be a large field, and it was removed from the PDI. The remaining segments were then integrated to obtain $\mathrm{PDI}_{\text {small fields. }}$. The overall proportion of dose from small fields was determined by comparing PDI ${ }_{\text {small fields }}$ and PDI ${ }_{0}$ pixel by pixel, and calculating the median proportion of dose in the in-field region (Equation 3.3).

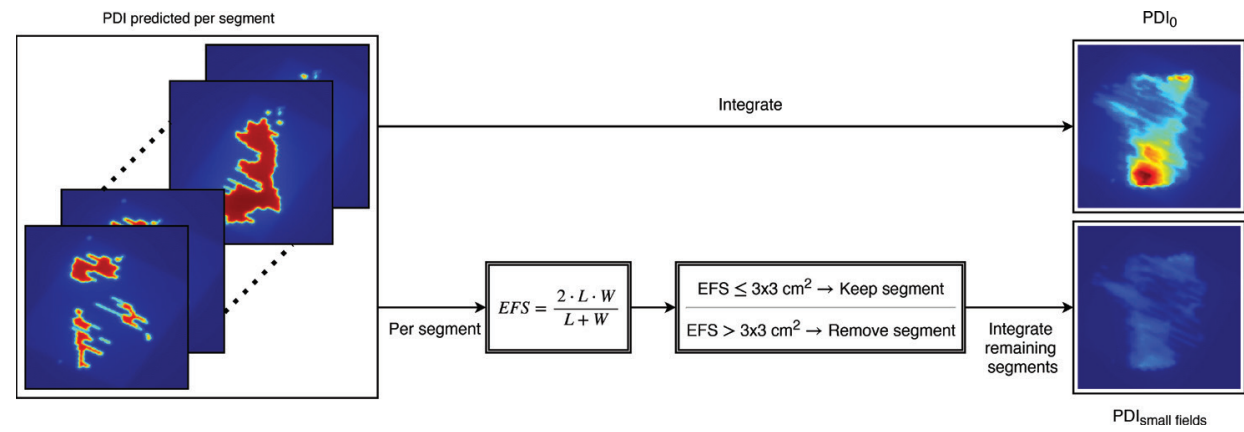

Figure 3.2: Creating $\mathrm{PDI}_{\text {small fields }}$ based on $\mathrm{PDI}_{0}$. EFS: equivalent field size; $\mathrm{PDI}_{0}$ : original portal dose image; $\mathrm{PDI}_{\text {small fields }}$ : portal dose image containing only dose from small fields. 


$$
\text { Overall proportion of dose from small fields }=\operatorname{median}\left(\frac{\mathrm{PDI}_{\text {small fields }}}{\operatorname{maxPDI}_{0}} \cdot 100\right)
$$

The uncertainty and proportion of dose from small fields were calculated for 109 VMAT arcs in total. Of these 109 arcs, 41 were from head and neck (H\&N) treatment plans, 33 from lung plans and 35 from prostate plans. A linear regression was performed to assess the relationship between the uncertainty and proportion of dose from small fields. The strength of the relationship was evaluated with Pearson's correlation coefficient $r$. A t-test was performed to determine the significance of the relationship.

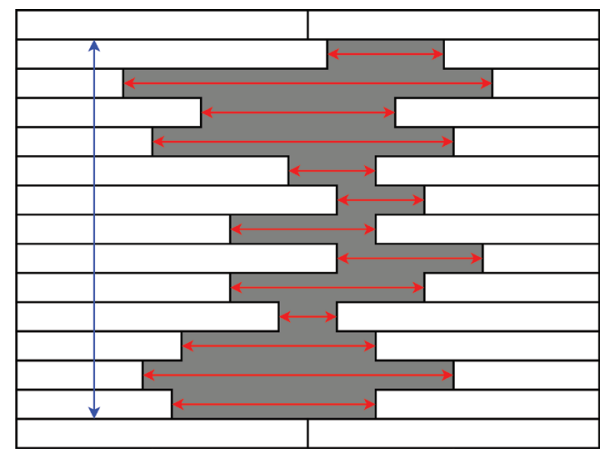

Figure 3.3: Schematic example of the MLC aperture of a VMAT segment. In the calculation of the equivalent field size, the blue arrow represents the length of the field $L$, whereas the average of the red arrows is the width of the field $\mathrm{W}$.
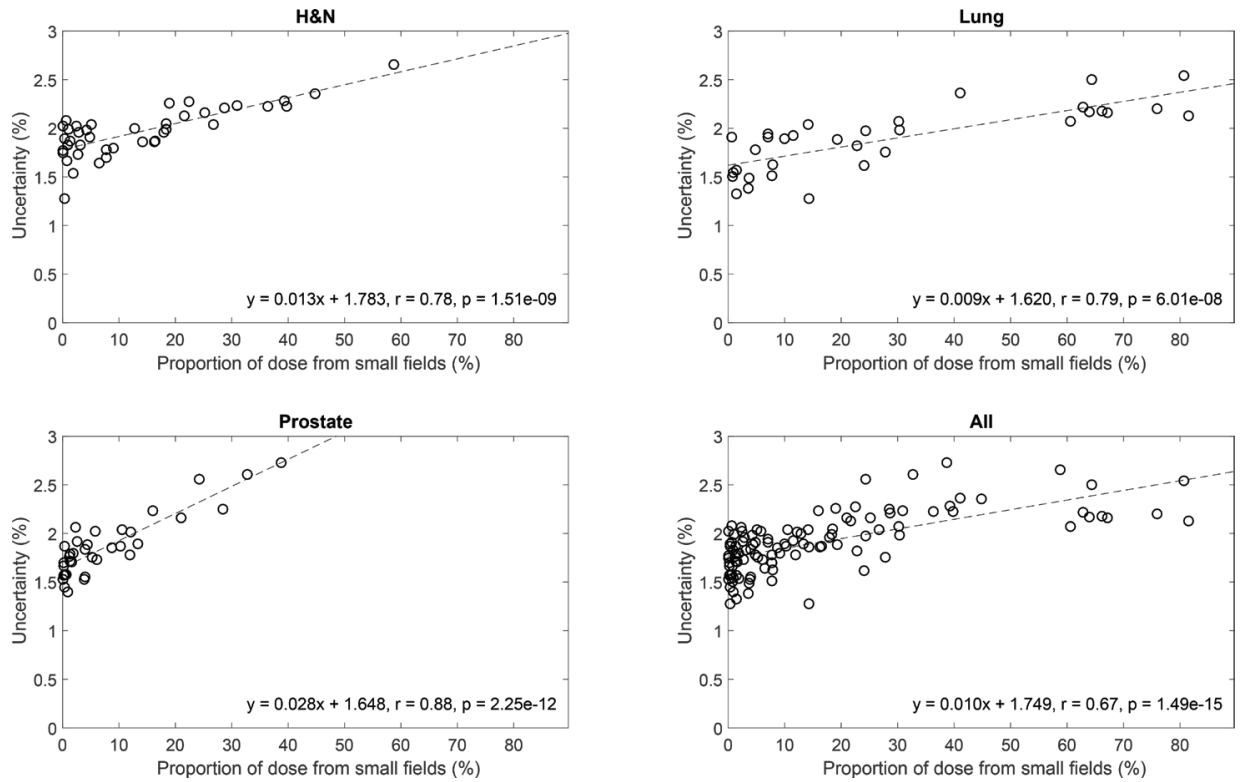

Figure 3.4: Relationship between the uncertainty of the dose prediction model and the proportion of dose from small fields for each treatment site separately, and for all sites together. The dashed line is the regression line. 


\section{Results}

Figure 3.4 shows the relationship between the overall uncertainty and the proportion of dose from small fields per tumor site and for all sites together. It can be seen that there is a statistically significant correlation in all cases (i.e., the slope is significantly different from 0 in all cases). However not all relationships are equally strong. The strongest correlation, based on Pearson's $r$, is found for the prostate cases, whereas the weakest occurs when all sites are considered together.

\section{Discussion}

The results show that there is a positive linear relationship between the uncertainty of a $2 \mathrm{D}$ pre-treatment dose prediction model and the proportion of dose from small fields in VMAT arcs. The maximum increase in uncertainty with an increasing proportion of dose from small fields is approximately one percentage point. The rate of this increase is dependent on the tumor site, and is probably caused by differences in field modulation for the different sites.

The model evaluated in this work can be used in a dose verification process. In such a process, typically gamma analysis with $(3 \%, 3 \mathrm{~mm})$ acceptance criteria is used to compare predicted and measured doses ${ }^{8}$. However, there is a tendency towards using stricter gamma criteria to be more sensitive to dose errors ${ }^{9-11}$. This in turn means that the uncertainty in the evaluated doses should be kept low, to avoid detecting noise instead of actual dose errors. Combining this with the fact that more small fields are used in VMAT, it may be wise to limit the dose coming from small fields in VMAT arcs, to decrease uncertainty in the dose verification process. Taking dose uncertainty into account, either for the measured dose or the dose prediction model, is not commonly done in gamma analysis. In this work the latter was quantified. In practice this should be combined with the uncertainty on the acquired dose, to decide on gamma acceptance criteria.

Although the model considered here is used for pre-treatment purposes, similar and more complex models are used for in vivo dose verification and adaptive radiotherapy ${ }^{12-17}$. A similar uncertainty analysis can be performed for these models, and the same logic can be extended to these situations. When making adaptive radiotherapy decisions based on dose differences smaller than $5 \%^{18-20}$, it is important to limit the uncertainty in the evaluated doses as much as possible. Only in that case it is possible to make well-informed decisions about treatment plan adaptation. It is likely that the occurrence of more small field segments in VMAT arcs has a similar influence on the uncertainty of more complex models.

As a limitation of this work, it should be noted that it is difficult to quantify the uncertainty and proportion of dose from small fields without generalizing too much. The calculation of the uncertainty and proportion of dose from small fields involve reducing a 2D comparison to one number. This may lead to some loss of information. Another issue may be the somewhat arbitrary definition of small fields, here chosen as an EFS of $3 \times 3 \mathrm{~cm}^{2}$. Different choices may lead to somewhat different numerical results for the uncertainties of the dose prediction model. For instance, when using an EFS threshold of $4 \times 4 \mathrm{~cm}^{2}$, the correlation coefficients for the individual tumor sites decrease slightly, but the correlation for all tumor sites together increases. 
To reduce the model uncertainties associated with dose from small fields, a recommendation could be to include small fields in the fitting process of dose verification models. Currently, as is common practice in commissioning as well, no fields smaller than $3 \times 3 \mathrm{~cm}^{2}$ are used. However, considering the difficulties involved in accurately measuring small fields, caution should be taken when including small field measurements. As the model uncertainty is based on the propagation of the uncertainty of the measurement set used for fitting the model, adding inaccurate small fields measurements could actually increase the uncertainty of the prediction model.

\section{Conclusion}

There is a positive linear relationship between the uncertainty and the proportion of dose from small fields for a 2D pre-treatment dose prediction model. It may be wise to limit the dose coming from small fields in VMAT arcs to decrease uncertainty in the dose verification process.

\section{Acknowledgments}

The authors would like to thank Dr. Stefan Scheib from Varian Medical Systems for commenting on the manuscript. This study was funded by Varian Medical Systems (project: TimePort-III). 


\section{References}

1. Teoh M, Clark C, Wood K, Whitaker S, and Nisbet A. Volumetric modulated arc therapy: A review of current literature and clinical use in practice. British Journal of Radiology, 2011. 84: p. 967-96.

2. Alfonso R, Andreo P, Capote R, Huq MS, Kilby W, Kjall P, Mackie TR, Palmans H, Rosser K, Seuntjens J, Ullrich W, and Vatnitsky S. A new formalism for reference dosimetry of small and nonstandard fields. Medical Physics, 2008. 35(11): p. 5179-86.

3. Das IJ, Ding GX, and Ahnesjo A. Small fields: Nonequilibrium radiation dosimetry. Medical Physics, 2008. 35(1): p. 206-15.

4. Fog LS, Rasmussen JF, Aznar M, Kjaer-Kristoffersen F, Vogelius IR, Engelholm SA, and Bangsgaard JP. A closer look at RapidArc radiosurgery plans using very small fields. Physics in Medicine and Biology, 2011. 56(6): p. 1853-63.

5. Swinnen ACC, Ollers MC, Roijen E, Nijsten SM, and Verhaegen F. Influence of the jaw tracking technique on the dose calculation accuracy of small field VMAT plans. Journal of Applied Clinical Medical Physics, 2017. 18(1): p. 186-95.

6. Baeza JA, Wolfs CJA, Nijsten SMJJG, and Verhaegen F. Validation and uncertainty analysis of a pre-treatment 2D dose prediction model. Physics in Medicine and Biology, 2018. 63(3): p. 035033.

7. Khan FM and Gibbons JP. Khan's The Physics of Radiation Therapy. 2014, Philadelphia, PA: Wolters Kluwer Health.

8. Low DA, Morele D, Chow P, Dou TH, and Ju T. Does the $\gamma$ dose distribution comparison technique default to the distance to agreement test in clinical dose distributions? Medical Physics, 2013. 40(7): p. 071722.

9. Heilemann G, Poppe B, and Laub W. On the sensitivity of common gamma-index evaluation methods to MLC misalignments in Rapidarc quality assurance. Medical Physics, 2013. 40(3): p. 031702.

10. Steers JM and Fraass BA. IMRT QA: Selecting gamma criteria based on error detection sensitivity. Medical physics, 2016. 43(4): p. 1982-94.

11. Maraghechi B, Davis J, Badu S, Fleck A, Darko J, and Osei E. Retrospective analysis of portal dosimetry pre-treatment quality assurance of prostate volumetric-modulated arc therapy (VMAT) plans. Journal of Radiotherapy in Practice, 2018. 17(1): p. 44-52.

12. van Elmpt WJC, Nijsten SMJJG, Mijnheer BJ, and Minken AWH. Experimental verification of a portal dose prediction model. Medical Physics, 2005. 32(9): p. 2805-18.

13. van Elmpt W, Nijsten SMJJG, Schiffeleers RFH, Dekker ALAJ, Mijnheer BJ, Lambin P, and Minken AWH. A Monte Carlo based three-dimensional dose reconstruction method derived from portal dose images. Medical Physics, 2006. 33(7): p. 2426-34.

14. Persoon LCGG, Nijsten SMJJG, Wilbrink FJ, Podesta M, Snaith JAD, Lustberg T, van Elmpt WJC, van Gils F, and Verhaegen F. Interfractional trend analysis of dose differences based on 2D transit portal dosimetry. Physics in Medicine and Biology, 2012. 57(20): p. 6445-58.

15. Greer P. 3D EPID based dosimetry for pre-treatment verification of VMAT-methods and challenges. Journal of Physics: Conference Series, 2013. 444(1): p. 012010.

16. Persoon LCGG, Egelmeer AGTM, Öllers MC, Nijsten SMJJG, Troost EGC, and Verhaegen F. First clinical results of adaptive radiotherapy based on 3D portal dosimetry for lung cancer patients with atelectasis treated with volumetric-modulated arc therapy (VMAT). Acta Oncologica, 2013. 52(7): p. 1484-89.

17. Spreeuw H, Rozendaal R, Olaciregui-Ruiz I, Gonzalez P, Mans A, Mijnheer B, and van Herk M. Online 3D EPID-based dose verification: Proof of concept. Medical Physics, 2016. 43(7): p. 3969-74.

18. Møller DS, Khalil AA, Knap MM, and Hoffmann L. Adaptive radiotherapy of lung cancer patients with pleural effusion or atelectasis. Radiotherapy and Oncology, 2014. 110(3): p. $517-22$. 
19. Nyeng TB, Nordsmark M, and Hoffmann L. Dosimetric evaluation of anatomical changes during treatment to identify criteria for adaptive radiotherapy in oesophageal cancer patients. Acta Oncologica, 2015. 54(9): p. 1467-73.

20. Zegers CML, Baeza JA, van Elmpt W, Murrer LHP, Verhoeven K, Boersma L, Verhaegen F, and Nijsten SMJJG. Three-dimensional dose evaluation in breast cancer patients to define decision criteria for adaptive radiotherapy. Acta Oncologica, 2017. 56(11): p. 1487-94. 



\section{Chapter 4}

\section{Detection of anatomical changes in lung cancer patients with 2D time-integrated, 2D time-resolved and 3D time-integrated portal dosimetry: A simulation study}

Authors

Cecile J.A. Wolfs*, Mariana G. Brás*, Lotte E.J.R. Schyns, Sebastiaan M.J.J.G. Nijsten, Wouter van Elmpt, Stefan G. Scheib, Christof Baltes, Mark Podesta and Frank Verhaegen

* These authors contributed equally

Adapted from

Physics in Medicine and Biology, 2017. 62(15): 6044-6061 


\begin{abstract}
The aim of this work is to assess the performance of 2D time-integrated (2D-TI), 2D time-resolved (2D-TR) and 3D time-integrated (3D-TI) portal dosimetry in detecting dose discrepancies between the planned and (simulated) delivered dose caused by simulated changes in the anatomy of lung cancer patients.

For six lung cancer patients, tumor shift, tumor regression and pleural effusion are simulated by modifying their CT images. Based on the modified CT images, timeintegrated ( $\mathrm{TI})$ and time-resolved (TR) portal dose images (PDIs) are simulated and 3DTI doses are calculated. The modified and original PDIs and 3D doses are compared by gamma analysis with various gamma criteria. Furthermore, the difference in the $\mathrm{D}_{95 \%}$ $\left(\Delta \mathrm{D}_{95 \%}\right)$ of the GTV is calculated and used as a gold standard. The correlation between the gamma fail rate and the $\Delta D_{95 \%}$ is investigated, as well the sensitivity and specificity of all combinations of portal dosimetry method, gamma criteria and gamma fail rate threshold.

On the individual patient level, there is a correlation between the gamma fail rate and the $\Delta D_{95 \%}$, which cannot be found at the group level. The sensitivity and specificity analysis showed that there is not one combination of portal dosimetry method, gamma criteria and gamma fail rate threshold that can detect all simulated anatomical changes.

This work shows that it will be more beneficial to relate portal dosimetry and DVH analysis on the patient level, rather than trying to quantify a relationship for a group of patients. With regards to optimizing sensitivity and specificity, different combinations of portal dosimetry method, gamma criteria and gamma fail rate should be used to optimally detect certain types of anatomical changes.
\end{abstract}




\section{Introduction}

The development of complex beam delivery techniques in external beam radiotherapy, such as intensity modulated radiotherapy (IMRT) and volumetric modulated arc therapy $(\mathrm{VMAT})^{1,2}$, has increased the need for accurate verification of the dose delivery. Due to the highly dynamic nature of VMAT, quality assurance is more difficult to perform than for conventional static radiation delivery techniques. The electronic portal imaging device (EPID) has been successfully used as a tool for performing dose verification for IMRT and VMAT; initially in a time-integrated ( $\mathrm{TI}$ ) manner, but more recently also in a time-resolved (TR) fashion ${ }^{3-7}$.

Besides being able to detect errors related to beam delivery or plan transfer in a pre-treatment setting, transit EPID dosimetry has been shown to be valuable in detecting errors at the patient level during treatment ${ }^{8-12}$. Anatomical changes occur commonly during the course of treatment, and can cause discrepancies between the planned and actually delivered dose distributions that may need corrective actions ${ }^{13,14}$. It is therefore important to perform dose verification during the patient's course of treatment to detect these errors and make the necessary adjustments to ensure treatment quality. Cone beam CT (CBCT) imaging can be used to detect anatomical changes as well. However, this modality does not allow to directly evaluate discrepancies in the delivered dose due to anatomical changes. Furthermore, with CBCT imaging additional dose is delivered to the patient, and additional scan time is needed. Transit portal dosimetry does not pose these disadvantages.

In this work, the performance of 2D time-integrated (2D-TI), 2D time-resolved (2D-TR) and 3D (in vivo) time-integrated (3D-TI) transit portal dosimetry are evaluated. In $2 \mathrm{D}$, the dose distribution behind the patient at the level of the portal imager is evaluated, whereas in 3D the dose inside the patient is calculated ${ }^{3}$. Furthermore, with TI portal dosimetry, the dose is accumulated over a VMAT arc, while TR portal dosimetry evaluates the dose distributions per VMAT segment (time between consecutive control points) ${ }^{6,12,15}$.

The focus of this work is on detecting dose discrepancies between the planned and (simulated) delivered dose caused by simulated changes in the patient's anatomy. Simulating anatomical changes and the resulting dose distributions allows for a systematic, high resolution analysis of the detection limits of portal dosimetry. The aim of this work is to determine these detection limits, and to discover trends that can ultimately aid in developing clinical decision models for dose guided adaptive radiotherapy.

\section{Materials and methods}

Tumor shift, tumor regression and pleural effusion are commonly occurring anatomical changes during treatment of lung cancer patients, and can cause errors in the delivered dose distributions ${ }^{16-18}$. To study the ability of portal dosimetry to detect these errors, these three types of anatomical changes were simulated using planning $\mathrm{CT}$ images of six lung cancer patients treated at MAASTRO Clinic. All patients were treated with VMAT treatments consisting of two (half-)arcs, using 6 or 10 MV photon beams delivered by a Varian TrueBeam linear accelerator (Varian Medical Systems, Palo 
Alto (CA), United States). Some characteristics of the patients and their treatment are outlined in Table 4.1.

\section{Anatomical change simulation}

Figure 4.1 illustrates the framework used in this work for simulating anatomical changes, simulating dose distributions and comparing the dose distributions. The simulation of anatomical changes was performed by modifying the patients' planning CT images with an in-house developed tool implemented in MATLAB (The Mathworks, Inc., Natick (MA), United States). All simulations and evaluations were based on the

Table 4.1: Characteristics of the six lung cancer patients and their treatments included in this study.

\begin{tabular}{lc}
\hline \multicolumn{2}{c}{ Patient and treatment characteristics } \\
\hline \hline Gender & $3 / 6$ \\
\hline Male & $3 / 6$ \\
Female & $75[68-85]$ \\
\hline Age (mean [range]) & \\
\hline Tumor location & $3 / 6$ \\
\hline Left lung & $3 / 6$ \\
Right lung & $3 / 6$ \\
\hline Upper lobe & $3 / 6$ \\
Lower lobe & $0 / 3$ \\
Middle lobe (Right lung only) & $31.5 \mathrm{~cm}^{3}[4.6-74.7] \mathrm{cm}^{3}$ \\
\hline Tumor volume (mean [range]) & \\
\hline Margins & \\
\hline GTV to CTV & $3 / 6$ \\
\hline 5 mm & $3 / 6$ \\
\hline Anisotropic ( 5 mm) & $1 / 6$ \\
\hline CTV to PTV (Individual margin based on internal target volume (ITV)) & $1 / 6$ \\
\hline 5 mm & $2 / 6$ \\
8 mm & $2 / 6$ \\
10 mm & $1 / 6$ \\
\hline Anisotropic ( 5 mm) & $2 / 6$ \\
\hline Dose prescription (dose per fraction (Gy) /number of fractions) \\
\hline $1.5 / 30$ & $1 / 6$ \\
$1.8 / 41$ or 43 & \\
$2.75 / 24$ & \\
\hline $.5 / 8$ & \\
\hline & \\
\hline
\end{tabular}


gross tumor volume (GTV), as this allowed for modifying and assessing a structure with visible boundaries rather than the invisible boundaries derived from extending the GTV to a clinical target volume (CTV) and planning target volume (PTV).

A baseline tumor shift was introduced by changing the position of the GTV. The voxels of the CT image where the tumor was located before shifting, were forced to a Hounsfield unit ( $\mathrm{HU}$ ) value of -750 , which was approximately the average of the HUs in the lungs of all patients. The shifts were simulated along the craniocaudal direction, as it has been shown previously by our group that shifts along this direction induce larger discrepancies (i.e., larger gamma fail rates) in measured portal dose distributions ${ }^{19}$. Thirteen consecutive shifts of $0.3 \mathrm{~cm}$ were applied resulting in a range of shifts from 0.3 to $3.9 \mathrm{~cm}$. These levels of severity were chosen because the voxel size of the CT in the craniocaudal direction was $0.3 \mathrm{~cm}$, whereas the maximum shift ensured the effect of moving the GTV (almost) completely out of the PTV was taken into account.

Tumor regression was simulated by applying a centroid-based deformation to the GTV. As with the simulation of tumor shift, the HUs of the voxels where the tumor was before the regression were forced to -750. The levels of regression simulated ranged from $10 \%$ to $90 \%$ of the original GTV volume, in steps of $10 \%$.

In the case of pleural effusion, gravity will often cause the fluid to accumulate on the dorsal side of the pleural cavity, as the patient is in supine position during treatment. Therefore, pleural effusion was simulated by creating a pleural effusion volume on the dorsal side of the lung the tumor was located in, and forcing the HUs in this volume to 0 (the value of water). The range of simulated pleural effusion levels was either from 0.5 to $4.0 \mathrm{~cm}$ in steps of $0.5 \mathrm{~cm}$, or from 0.1 to $0.8 \mathrm{~cm}$ in steps of $0.1 \mathrm{~cm}$, depending on whether the tumor was located at the ventral side of the lung or more towards the dorsal side, respectively.

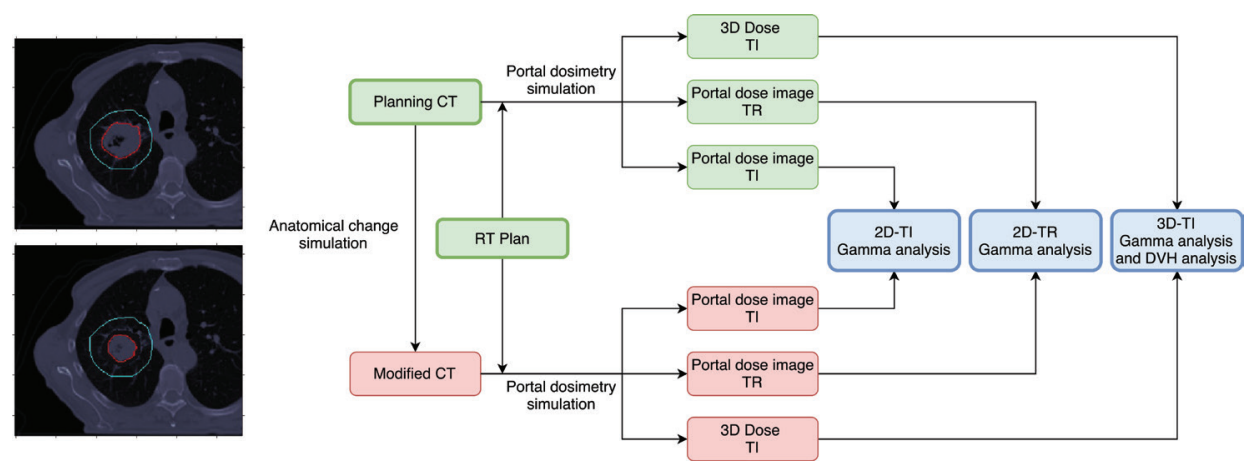

Figure 4.1: A flowchart of the process of simulating anatomical changes, simulating dose distributions and comparing the dose distributions used in this work. The bold outlined boxes on the left and right represent the input and output, respectively. The boxes in green depict all data not containing simulated anatomical changes, while those in red do contain (the results of) simulated anatomical changes. The blue boxes represent the comparisons between the simulated dose distributions. On the far left an example of simulated tumor regression (50\%) is displayed, with the planning CT (top) and the modified CT (bottom). The GTV is delineated in red, the PTV (for reference, not modified) in blue. 
After introducing anatomical changes of different magnitudes in each patient's planning $\mathrm{CT}, 2 \mathrm{D}$ portal dose images (PDIs), at a source-to-detector distance (SDD) of $150 \mathrm{~cm}$, were simulated for the original planning CTs and for each modified CT, with an in-house developed portal dose prediction mode ${ }^{20}$. All PDI predictions were performed based on each patient's original RT plan. Furthermore, 3D doses in the patients were calculated with a Monte Carlo based dose reconstruction method ${ }^{21}$. In $2 \mathrm{D}$, both $\mathrm{TI}$ and TR PDIs were predicted, while in 3D only TI dose distributions were simulated.

The 2D PDIs and 3D dose distributions of the original planning CT and those of the modified CTs were compared using a global gamma analysis ${ }^{22,23}$. For 2D-TR portal dosimetry a TR gamma analysis was used ${ }^{24}$. As the data is simulated, there is perfect synchronization in time between the original and modified data, meaning that the time of interest parameter in this analysis was set to 0 .

The most commonly used gamma criteria for dose difference and distance to agreement are $(3 \%, 3 \mathrm{~mm})^{25}$. Yet, studies have indicated that these criteria are not sensitive enough to detect relevant dose discrepancies ${ }^{26-28}$. To study the performance of various gamma criteria in detecting errors, six sets of gamma criteria were employed, namely $(1 \%, 1 \mathrm{~mm}),(1.5 \%, 1.5 \mathrm{~mm}),(2 \%, 2 \mathrm{~mm}),(2.5 \%, 2.5 \mathrm{~mm}),(3 \%, 3 \mathrm{~mm})$ and $(5 \%, 3 \mathrm{~mm})$. The results of the global gamma analyses are expressed in terms of the gamma fail rate, which is the percentage of failing pixels (i.e., pixels with $|\gamma|>1$ ) inside the radiation field in 2D (accumulated over the VMAT arc for TI and per segment for TR), and the percentage of failing voxels inside the GTV structure in 3D. To evaluate the performance of the three portal dosimetry methods (2D-TI, 2D-TR and 3D-TI) in detecting the simulated anatomical changes, it was first determined for which cases the gamma fail rate exceeded $10 \%$, which is considered a clinically acceptable threshold for detecting errors in the delivered dose at our clinic. The analysis can be repeated with another value for this parameter.

\section{Correlation between DVH metrics and gamma fail rate}

In clinical practice, dose-volume histogram (DVH) analysis is an important part of treatment plan optimization and comparison ${ }^{29}$. In this work, it was considered the gold standard to detect relevant dose discrepancies. For the design of decision support protocols for adaptive dose guided radiotherapy and to prevent subjective decision making, a quantitative relationship between gamma analysis results and DVH metrics should be established. Therefore, DVHs were generated for each 3D-TI dose distribution. The DVH metric considered in this work is the dose to $95 \%\left(D_{95 \%}\right)$ of the GTV. The percentage of change in this metric in comparison to the original GTV was determined as follows:

$$
\Delta \mathrm{D}_{95 \%}=\left|\frac{\mathrm{D}_{95 \%}(\text { modified GTV })-\mathrm{D}_{95 \%}(\text { original GTV })}{\mathrm{D}_{95 \%}(\text { original GTV })}\right| \cdot 100
$$

The correlation between the $\Delta D_{95 \%}$ and the gamma fail rate was investigated for all three portal dosimetry methods and for all six sets of gamma criteria. The correlation analysis consisted of calculating Pearson's $r$ for each patient separately, and for the six patients as a group. The correlation coefficient is interpreted as representing no 
linear relationship for $r$ between 0 and \pm 0.2 , a weak relationship for $r$ between \pm 0.2 and \pm 0.5 , a moderate relationship for $r$ between \pm 0.5 and \pm 0.8 and a strong relationship for $r$ between \pm 0.8 and $\pm 1^{30}$. In the case of tumor shift, a saturation-growth curve $y=a \frac{x}{b+x}$ was fitted to the data, and this non-linear fit was assessed by the coefficient of determination $R^{2}$.

\section{Sensitivity and specificity analysis}

Sensitivity and specificity analysis using receiver operating characteristic (ROC) curves is widely used to determine the performance of a dichotomous diagnostic test against a gold standard ${ }^{31}$. In this case, the diagnostic tests were the portal dosimetry methods with their resulting gamma fail rates, which can be either positive (i.e., exceeding a certain threshold) or negative (i.e., not exceeding a certain threshold). As discussed in the previous section, DVH analysis, and in this case the $\Delta \mathrm{D}_{95 \%}$ of the GTV, was considered the gold standard. This metric was deemed positive, and to represent a relevant dose deviation due to the simulated anatomical change, if it exceeded $4 \% 32$, and negative otherwise. With this information, it could be established whether, for instance, a positive gamma fail rate result coincides with a positive $\Delta \mathrm{D}_{95 \%}$, constituting a true positive result. In a similar way, true negatives (both gamma fail rate and $\Delta D_{95 \%}$ negative), false positives (gamma fail rate positive but $\Delta D_{95 \%}$ negative) and false negatives (gamma fail rate negative but $\Delta \mathrm{D}_{95 \%}$ positive) could be determined.

Based on these definitions, confusion matrices containing the number of true positives, true negatives, false positives and false negatives can be created, from which the sensitivity (true positive rate) and specificity (true negative rate) can be calculated. ROC curves are a graphical display of the sensitivity and (1-specificity) over a range of thresholds of the diagnostic test value, which was the gamma fail rate in this work. Therefore, ROC curves were generated in MATLAB for each combination of portal dosimetry method and gamma criteria. For each ROC curve the area under the curve (AUC) was calculated, as this is a measure of test validity ${ }^{31}$. Hence, the combination of portal dosimetry method and gamma criteria with the highest AUC was considered to be the one that could detect relevant anatomical changes most accurately.

To establish which gamma fail rate threshold maximizes the correct classification of relevant and irrelevant dose deviations due to anatomical changes, the optimal cut-off point on each ROC curve was determined. This point was taken as the point geometrically closest to $(0,1)$, where both sensitivity and specificity are $1^{31}$. The threshold corresponding to this cut-off point was taken as the optimal gamma fail rate threshold.

\section{Results}

\section{Anatomical change simulation}

Figure 4.2 shows the DVH curves for each level of simulated tumor shift, tumor regression and pleural effusion for one patient. In the case of simulated tumor shift, the DVH curves in Figure 4.2a show that the larger the shift, the more the distinct corner at the top right is disappearing. This is due to the GTV shifting away from the high dose region, which means that the tumor volume receiving a high dose decreases. 

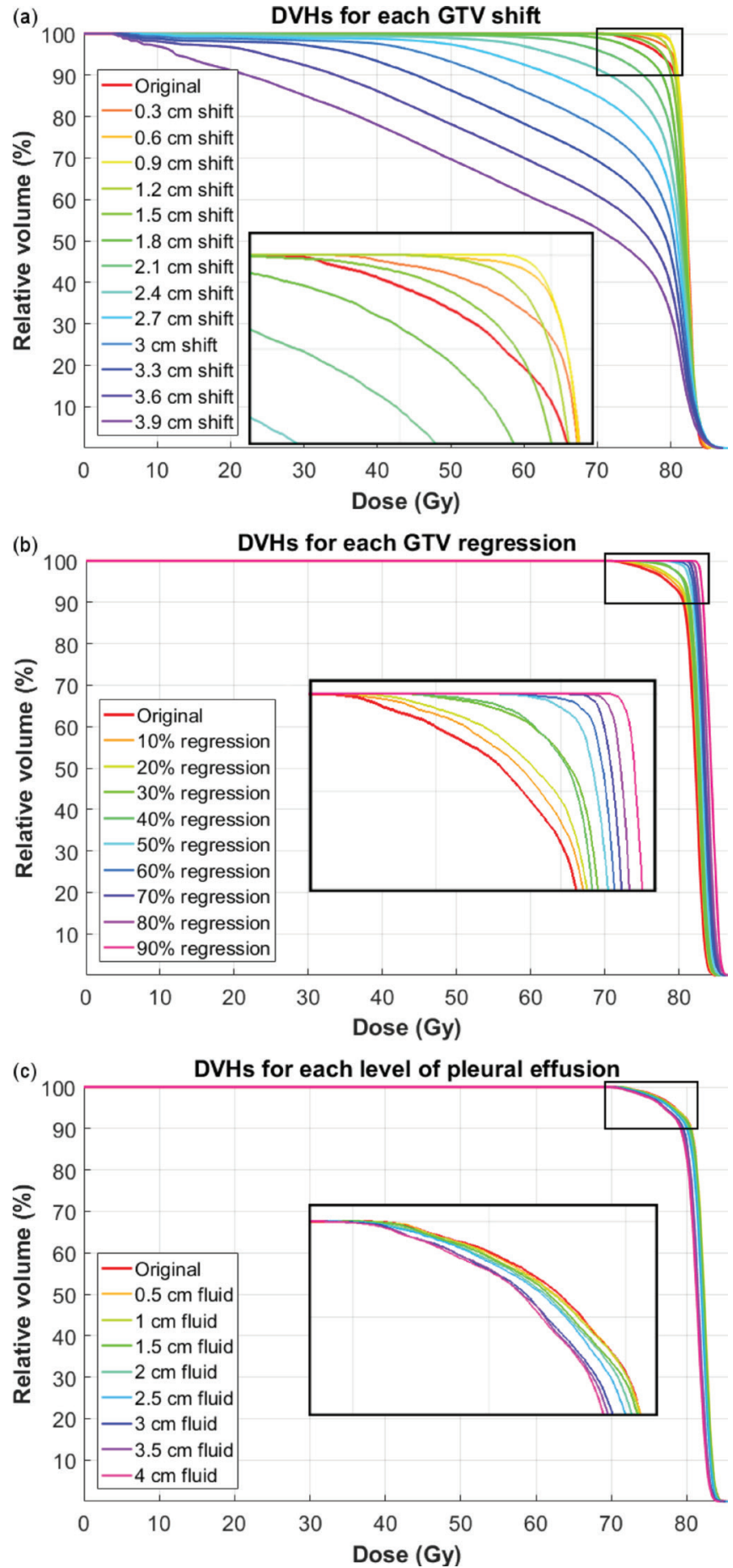

Figure 4.2: DVH curves for the GTV of one patient, for a) tumor shift, b) tumor regression and c) pleural effusion. 
For this patient, there is first a slight improvement in the DVH curves before this effect occurs. This is because the dose is not homogeneously distributed over the GTV, due to nearby organs-at-risk (OARs) that needed to be avoided. By shifting the GTV, it is first moved into a slightly higher dose region, where it is more uniformly covered, before showing the expected decrease in volume receiving high dose. This occurs in three out of the six patients analyzed. For the other three patients the expected decrease in the volume receiving high dose is visible immediately. The changes in $D_{95 \%}$ observed for tumor shift are generally large, and can even be up to $95 \%$ for the largest shifts and smaller tumor volumes.

The DVH curves corresponding to tumor regression in Figure 4.2b show the opposite effect. There, the dose to the GTV seems to become higher with increasing tumor regression. This is because high density tumor tissue is replaced by low density lung tissue all around the shrinking tumor. Therefore, there is less attenuation from all sides of the tumor, which increases the dose to the GTV. For all patients, the same change in DVH curves is seen, albeit slightly larger for the three patients for which the GTV was not homogeneously covered in the original situation. For these three patients, as the tumor regression increases, a larger proportion of the relative tumor volume receives a high dose, as a larger part of the GTV is uniformly covered in the high dose region. The largest change in $D_{95 \%}$ that occurs for tumor regression is approximately $6 \%$.

In the DVHs for pleural effusion, shown in Figure 4.2c, it can be seen that the curves move slightly to lower doses with increasing level of pleural effusion. Like tumor shift, the volume of the GTV receiving high dose is decreasing. However, in this case this is due to the pleural effusion fluid attenuating the posterior beams more than lung tissue would, hence there is less dose reaching the tumor. Even though the beams do pass through the simulated pleural effusion volume in all patients (the half-arcs go from $180^{\circ}$ to $0^{\circ}$, the full arcs from $180^{\circ}$ back to $180^{\circ}$ ), the attenuation only happens in a small part of the $180^{\circ}$ or $360^{\circ}$ VMAT arc. In fact, for pleural effusion, no relevant changes (i.e., $>4 \%$ ) in the $D_{95 \%}$ are observed, with the largest change being $2 \%$.

Figure 4.3 shows 3D bar charts for the number of patients for which each method can detect the simulated anatomical changes, for the various gamma criteria. It can be seen that in all cases, different methods can detect different levels of the simulated anatomical changes, and the gamma criteria that are used also influence this.

As is apparent from Figure 4.3a, the 2D-TI method can detect simulated tumor shift in most patients for tight gamma criteria, such as $(1 \%, 1 \mathrm{~mm})$, except for the smallest shifts. However, its performance decreases quickly for looser gamma criteria. 2D-TR shows this trend as well, although it is able to detect deviations in more patients than 2D-TI for the intermediate gamma criteria. 3D-TI performs best in detecting simulated tumor shift, but for looser gamma criteria it can only detect the larger shifts. From the total amount of simulated shifts for all patients and gamma criteria, which was 468 , 2D-TI could detect $29.5 \%$, 2D-TR detected $38.7 \%$ and 3D-TI outperformed the other methods by detecting $78.2 \%$.

In Figure 4.3b, it can be seen that 3D-TI outperforms 2D-TI and 2D-TR in detecting tumor regression, for tight gamma criteria. For looser criteria none of the methods perform well, with 3D-TI not detecting the tumor regressions in any of the 

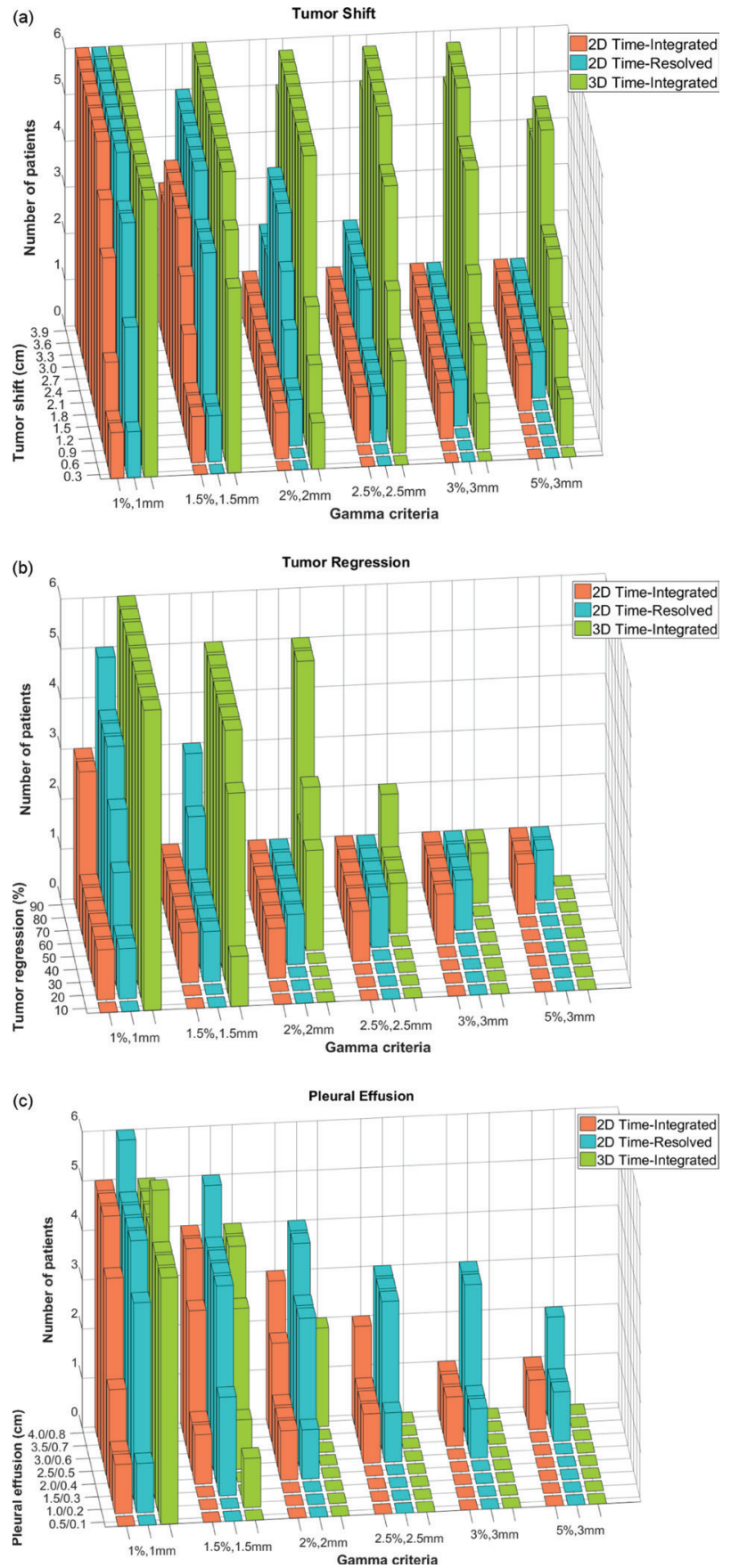

Figure 4.3: $3 \mathrm{D}$ bar charts of the number of patients for which the gamma fail rate exceeds the $10 \%$ threshold for each simulated anatomical change and for different gamma criteria, for a) tumor shift, b) tumor regression and c) pleural effusion. For pleural effusion there were two options for the simulated levels: $0.5-4 \mathrm{~cm}$ or $0.1-1$ $\mathrm{cm}$. Both of these options are taken together in this graph, as the pleural effusion level relative to the tumor position is similar. 
patients for the $(5 \%, 3 \mathrm{~mm})$ gamma criteria. For $(1 \%, 1 \mathrm{~mm}), 2 \mathrm{D}-\mathrm{TR}$ is able to detect deviations in more patients than 2D-TI, but for looser gamma criteria their performance is similar. Looking at the percentage of simulated regressions the different methods detected from a total of 324, 2D-TI, 2D-TR and 3D-TI detected $12.3 \%, 15.7 \%$ and $36.4 \%$, respectively.

Figure $4.3 \mathrm{c}$ shows that for the tightest gamma criteria simulated pleural effusion can be detected in most patients, especially using 2D-TR or 3D-TI. For looser gamma criteria, 3D-TI cannot detect deviations in any of the patients, while 2D-TR performs better than 2D-TI in these cases. The total amount of simulated pleural effusion cases was 288, of which 2D-TI detected 18.8\%, 2D-TR 31.6\% and 3D-TI 19.4\%.

Generally, except for pleural effusion, the simulated anatomical changes are large enough to cause significant deviations in the $\mathrm{D}_{95 \%}$. 3D-TI is able to detect errors in more cases than 2D-TI and 2D-TR. For tumor regression and pleural effusion, 3D-TI detects smaller anatomical changes when tighter gamma criteria are used, while for looser criteria the 2D methods are better.

\section{Correlation between DVH metrics and gamma fail rate}

As outlined previously, a quantitative relationship between the outcomes of the portal dosimetry methods (i.e., gamma fail rates) and DVH metrics should be established. Figure 4.4 shows the results of the correlation analysis within a single patient (the same patient for which the DVH curves are shown in Figure 4.2) for one of the sets of gamma criteria evaluated. It can be seen that the correlation is generally strong, especially for tumor regression and pleural effusion. For tumor shift, there was a clear non-linear relationship in the gamma fail rate vs. $\Delta \mathrm{D}_{95 \%}$ curve, as this curve starts to saturate when the GTV shifts outside the PTV. This effect is seen for all patients, with the onset of the saturation dependent on the magnitude of the shift needed to move the tumor out
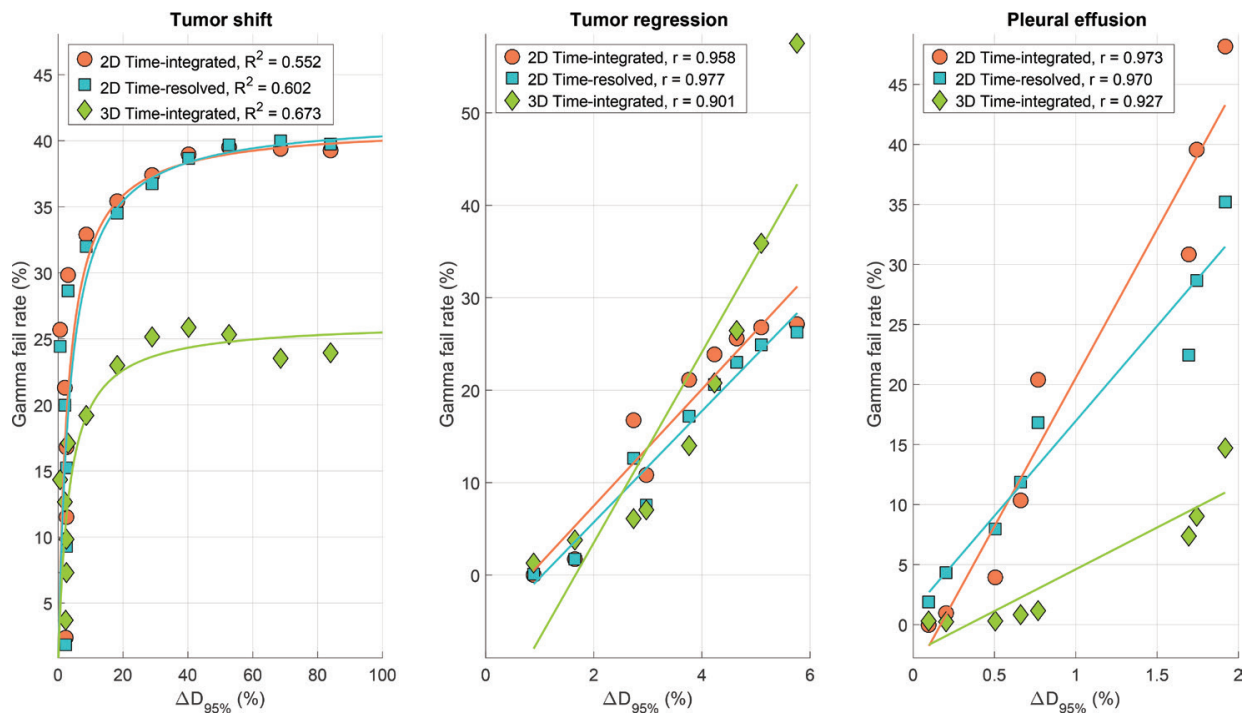

Figure 4.4: Correlation between $\Delta \mathrm{D}_{95 \%}$ and gamma fail rate for one individual patient, for gamma criteria of $(2 \%, 2 \mathrm{~mm})$. For tumor shift the relationship was fitted to a saturation-growth curve. 

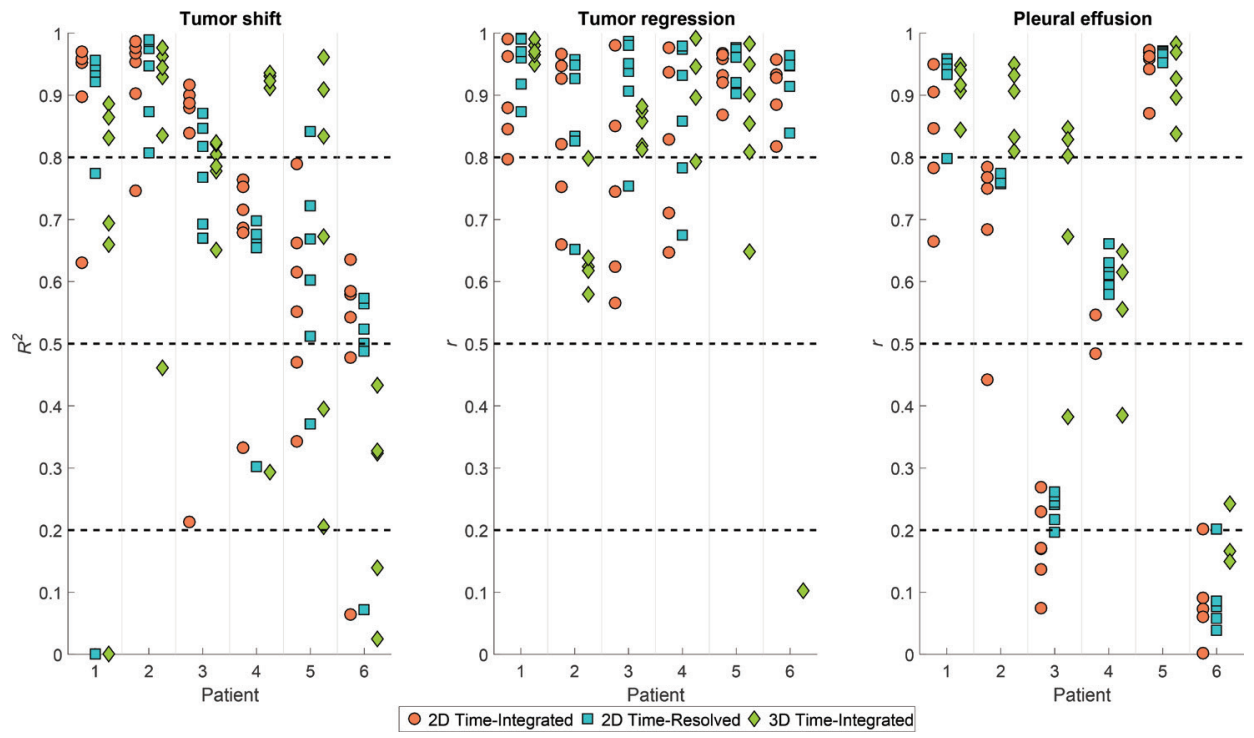

Figure 4.5: Correlation coefficients for all patients individually. Each marker represents the correlation coefficient $r$ or coefficient of determination $R^{2}$ for a certain patient, portal dosimetry method and gamma criteria. If there are less than 6 markers (one for each set of gamma criteria) per method per patient, $r$ or $R^{2}$ could not be calculated for all gamma criteria. The dashed lines indicate the regions in which the coefficients represent a non-existent, weak, moderate and strong linear relationship. Patient 5 is the patient whose individual results are displayed in Figure 4.4. Not displayed are the weak negative correlation coefficients for patient 6 in the case of tumor regression.
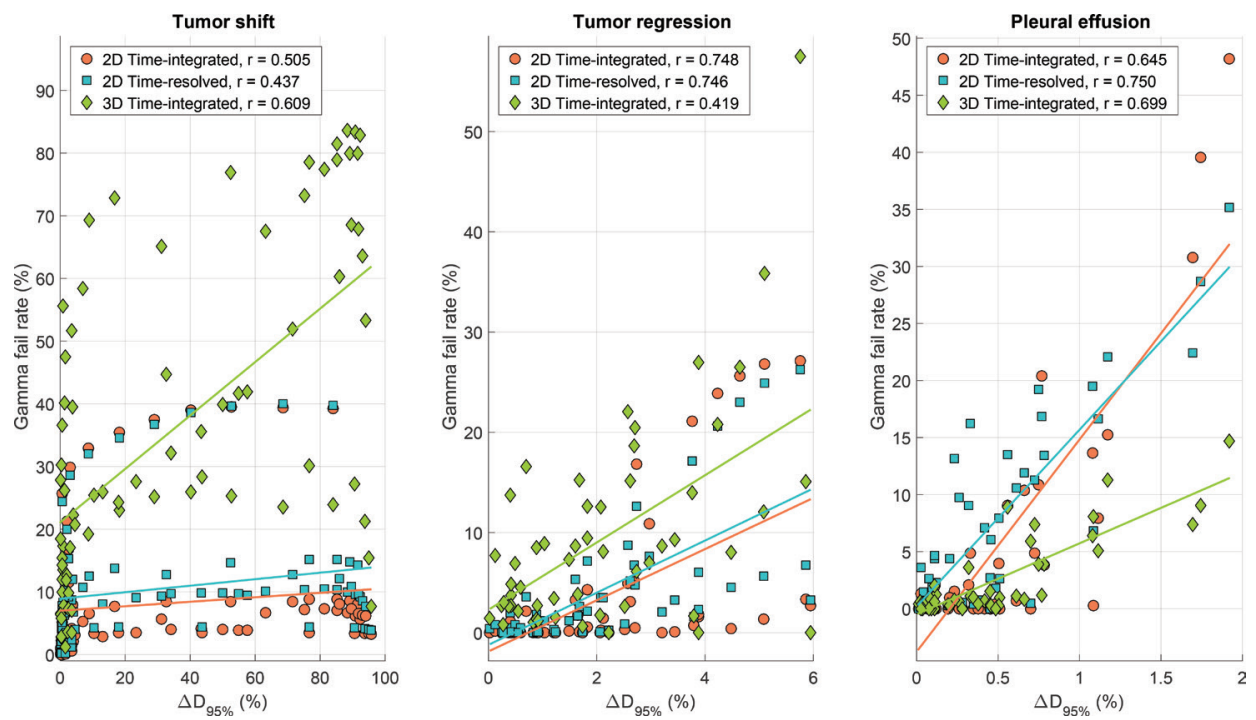

Figure 4.6: Correlation between $\Delta \mathrm{D}_{95 \%}$ and the gamma fail rate for the six patients as a group, for gamma criteria of $(2 \%, 2 \mathrm{~mm})$. 
of the PTV, and the rate of saturation dependent on the tumor volume. Therefore, a non-linear fit was performed using a saturation-growth curve. For all three types of anatomical changes, similar trends were observed for the other gamma criteria.

An overview of the correlation coefficients for all patients on an individual level is provided in Figure 4.5. Generally, there is a stronger relationship for tumor regression than for shift and pleural effusion. For all simulated anatomical changes, there are several cases in which the correlation coefficient or coefficient of determination could not be calculated. This occurs when the portal dosimetry method did not detect any errors, for instance with simulated pleural effusion, 3D-TI and gamma criteria of (5\%, $3 \mathrm{~mm})$. Generally, this occurred more frequently for looser gamma criteria. For most patients there are a number of cases in which there is a strong relation between the gamma fail rate and $\Delta \mathrm{D}_{95 \%}$. However, there are also cases for which the correlation is weak or even non-existent, such as for pleural effusion in patient 6.

When looking at the six patients as a group, the relationship between the gamma fail rate and $\Delta D_{95 \%}$ is less strong (Figure 4.6). Especially in the case of tumor shift, the correlation is non-existent for 2D, and only moderate for 3D. For tumor regression, the correlation is also moderate. There seems to be a stronger correlation for pleural effusion. However, as mentioned previously, there are no significant deviations ( $>4 \%)$ in the $D_{95 \%}$ for this type of simulated anatomical change. When applying the other gamma criteria, these trends are equivalent.

\section{Sensitivity and specificity analysis}

The sensitivity and specificity analysis was performed for tumor shift and tumor regression only, since there were no significant deviations in the $D_{95 \%}$ for pleural effusion. For this analysis, the data of all patients with respect to an anatomical change type is combined. A true positive result occurs when both the gamma fail rate and the $D_{95 \%}$ deviate significantly, i.e., the gamma fail rate exceeds a certain threshold in the infield region in $2 \mathrm{D}$ or in the GTV structure in $3 \mathrm{D}$, and $\Delta \mathrm{D}_{95 \%}$ is larger than $4 \%$.

Figure 4.7 shows the ROC curves and AUC values for simulated tumor shift. Based on the AUC values, when the gamma criteria are tight, 2D-TI performs slightly better than 2D-TR, but when the gamma criteria are looser, 2D-TR does much better. Moreover, the method that performs best is 3D-TI, particularly with gamma criteria of $(2 \%, 2 \mathrm{~mm})$ or $(2.5 \%, 2.5 \mathrm{~mm})$. Based on the optimal cut-off points on the ROC curves, the corresponding gamma fail rate thresholds for this best-performing method with these gamma criteria, are $19 \%$ and $12 \%$, respectively. The confusion matrix for these optimal cases, displayed in Table 4.2a, shows the achievable frequencies of correct classifications of relevant simulated tumor shift. This confusion matrix applies both to $3 \mathrm{D}-\mathrm{TI}$ with gamma criteria $(2 \%, 2 \mathrm{~mm})$ and fail rate threshold $19 \%$ and to $3 \mathrm{D}-\mathrm{TI}$ with $(2.5 \%, 2.5 \mathrm{~mm})$ and $12 \%$. The sensitivity and specificity are $93.6 \%$ and $71.0 \%$, respectively.

A similar analysis for tumor regression, displayed in Figure 4.8, reveals that 2DTR has the most stable performance over all gamma criteria, i.e., its AUC changes the least for different gamma criteria. Especially for the $(5 \%, 3 \mathrm{~mm})$ criteria the performance of 2D-TI and 3D-TI is far worse than that of 2D-TR. This is also visible in the ROC curve for the $(5 \%, 3 \mathrm{~mm})$ criteria. With 2D-TI and 3D-TI for these criteria, there are very few cases for which the gamma fail rate is larger than 0 . Thus, only a few thresholds can 
be set to obtain the ROC curves. The optimal combination of method, gamma criteria and gamma fail rate threshold, is 2D-TR with $(1 \%, 1 \mathrm{~mm})$ criteria, and a threshold of $18 \%$. For this combination, for which the confusion matrix is shown in Table $4.2 \mathrm{~b}$, the sensitivity and specificity are $75.0 \%$ and $93.5 \%$, respectively.

To compare with the commonly used $(3 \%, 3 \mathrm{~mm})$ gamma criteria and $10 \%$ gamma fail rate threshold, in Table 4.3 the sensitivity and specificity for each method with these criteria and fail rate threshold are displayed. Except for 3D-TI and tumor shift, the sensitivity is low, meaning that there are many false negatives when these parameters are used. On the contrary, the specificity is high, so there are not many false positive cases. Especially for tumor regression there is a large difference in the sensitivity that can be achieved.
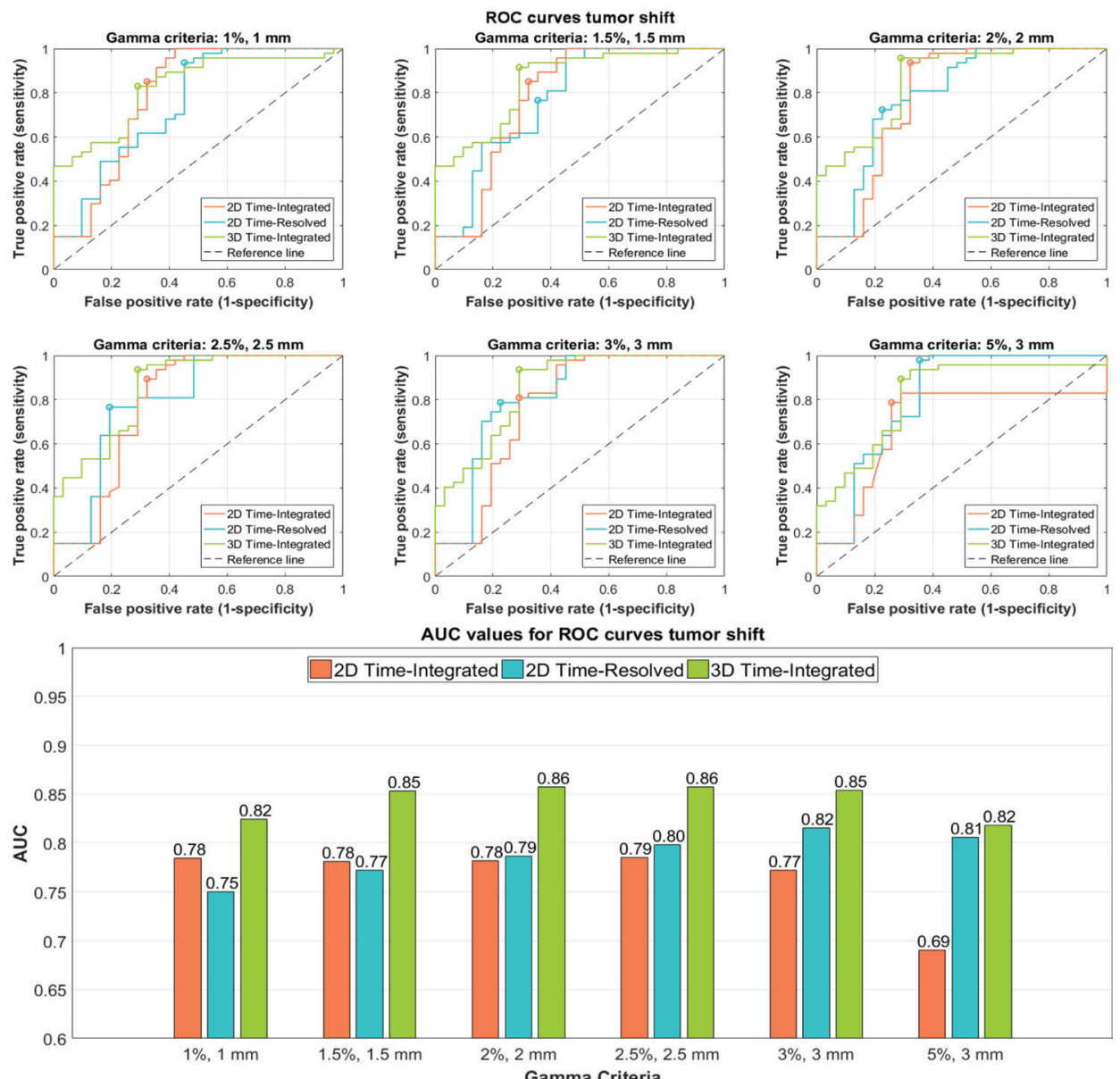

Figure 4.7: ROC curves and AUC values for tumor shift. The markers on the ROC curves represent the optimal cut-off point, i.e., the point on each curve closest to $(0,1)$. 

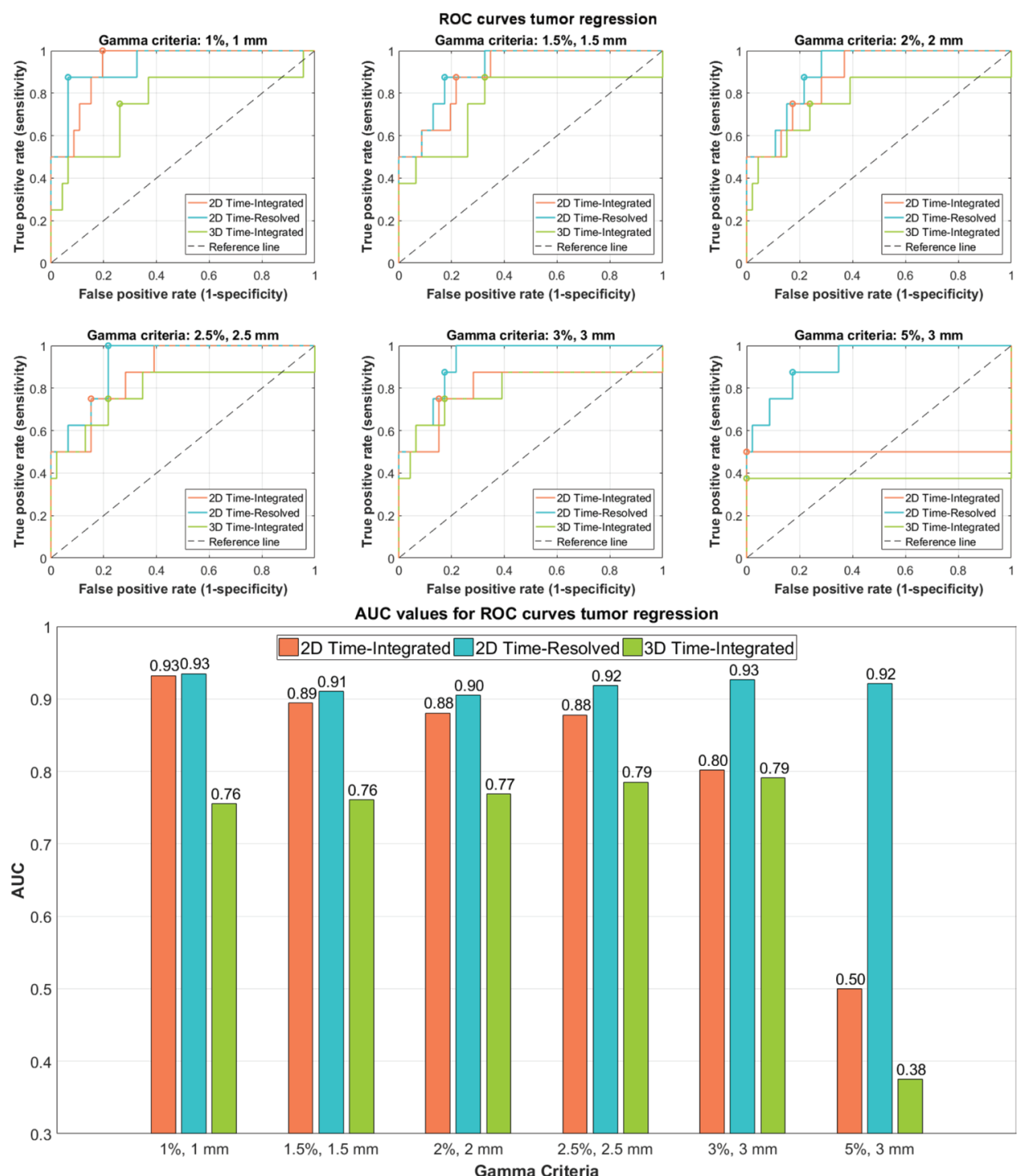

Figure 4.8: ROC curves and AUC values for tumor regression. The markers on the ROC curves represent the optimal cut-off point, i.e., the point closest to $(0,1)$. 
Table 4.2: Confusion matrices (displaying the frequency of positive and negative detection rates) for the optimal methods for detecting anatomical changes: a) 3D-TI with gamma criteria (2\%, $2 \mathrm{~mm})$ and gamma fail rate threshold of $19 \%$, or with gamma criteria $(2.5 \%, 2.5 \mathrm{~mm})$ and gamma fail rate threshold of $12 \%$ for tumor shift (their confusion matrices are exactly the same), and b) 2D-TR with gamma criteria (1\%, $1 \mathrm{~mm})$ and gamma fail rate threshold of $18 \%$ for tumor regression.

a)

\begin{tabular}{|c|c|c|c|c|c|c|c|}
\hline \multirow{2}{*}{\multicolumn{2}{|c|}{$\begin{array}{c}\text { Optimal confusion } \\
\text { matrix } \\
\text { Tumor shift }\end{array}$}} & \multicolumn{2}{|c|}{ DVH analysis } & \multirow{2}{*}{\multicolumn{2}{|c|}{$\begin{array}{l}\text { Optimal confusion } \\
\text { matrix } \\
\text { Tumor regression }\end{array}$}} & \multicolumn{2}{|c|}{ DVH analysis } \\
\hline & & \multirow{2}{*}{$\frac{\text { Positive }}{44}$} & \multirow{2}{*}{$\begin{array}{c}\text { Negative } \\
9\end{array}$} & & & \multirow{2}{*}{$\frac{\text { Positive }}{6}$} & \multirow{2}{*}{$\frac{\text { Negative }}{3}$} \\
\hline Gamma & Positive & & & \multirow{2}{*}{$\begin{array}{l}\text { Gamma } \\
\text { analysis }\end{array}$} & Positive & & \\
\hline & Negative & 3 & 22 & & Negative & 2 & 43 \\
\hline
\end{tabular}

Table 4.3: The sensitivity and specificity for simulated anatomical changes (tumor shift and tumor regression) and each portal dosimetry method, for gamma criteria $(3 \%, 3 \mathrm{~mm}$ ) and a gamma fail rate threshold of $10 \%$. The sensitivity and specificity of the optimal combinations of portal dosimetry method, gamma criteria and gamma fail rate threshold (see Table 4.2) are displayed as well for comparison.

\begin{tabular}{|c|c|c|c|c|c|c|c|c|}
\hline & \multirow{2}{*}{\multicolumn{4}{|c|}{ Tumor shift }} & & & & \\
\hline & & & & & \multicolumn{4}{|c|}{ Tumor regression } \\
\hline & 2D-TI & 2D-TR & 3D-TI & Optimal & 2D-TI & 2D-TR & 3D-TI & Optimal \\
\hline Sensitivity (\%) & 14.9 & 14.9 & 91.5 & 93.6 & 50 & 50 & 25 & 75 \\
\hline Specificity (\%) & 87.1 & 90.3 & 71.0 & 71.0 & 91.8 & 100 & 100 & 93.5 \\
\hline
\end{tabular}

\section{Discussion}

It is evident that there is a difference in the ability of 2D-TI, 2D-TR and 3D-TI portal dosimetry to detect different simulated anatomical changes. Their performance also depends on the gamma criteria that are used, as more errors are detected with tighter gamma criteria. Naturally, not all of the dose deviations detected with the portal dosimetry methods are clinically relevant, and it needs to be established which ones are, also taking the uncertainties of the methods into account when using them for actual measurements instead of simulations.

The most important result of this work is that there is a relationship at the patient level between the gamma fail rate and $\Delta D_{95 \%}$, for many combinations of portal dosimetry methods and gamma criteria. However, at the cohort level the relationship is less strong, and in many cases non-existent. This implies that it will be more beneficial to relate portal dosimetry and DVH analysis on the patient level rather than trying to quantify this relationship for a group of patients, as has been done previously ${ }^{19,33,34}$.

Based on these results, in clinical practice, for each patient, gamma fail rate vs. $\Delta \mathrm{D}_{95 \%}$ curves could be simulated for certain anatomical changes prior to treatment. These simulated curves could then serve to decide when the gamma fail rate measured during treatment exceeds a level that indicates a clinically relevant change in the $D_{95 \%}$. As the relationship between the gamma fail rate and the $\Delta D_{95 \%}$ is not the same for all anatomical changes, simulating a curve for each type of anatomical change might be necessary. 
Although this work focused on the $\mathrm{D}_{95 \%}$, the correlation between other DVH metrics and the gamma fail rate on the patient level should be investigated as well. For instance, $\mathrm{D}_{\text {mean }}$ and $\mathrm{V}_{95 \%}$ change in a similar manner to the $\mathrm{D}_{95 \%}$ for the anatomical changes simulated in this study, and thus likely correlate with the gamma fail rate as well for individual patients. Other measures, such as dose conformity, could additionally be evaluated.

Furthermore, evaluating and relating DVH metrics for OARs to the gamma fail rate could provide additional insight, as the simulated anatomical changes in this study do not only influence the dose to the GTV, but also the dose to, for instance, the healthy lung tissue. The constraints for the OARs used during treatment planning are potentially violated due to anatomical changes. A thorough analysis of the effect of anatomical changes on the OARs could aid in developing criteria to decide when to adapt a treatment plan.

The sensitivity and specificity analyses provide information on which combination of portal dosimetry method, gamma criteria and gamma fail rate threshold to use to detect certain types of anatomical changes with optimal sensitivity and specificity. It should be noted that the value of $\Delta \mathrm{D}_{95 \%}$ that is considered significant influences the outcomes of these analyses. Moreover, in this work the optimal threshold was found in a geometrical way from the ROC curve, while in clinical practice a prespecified sensitivity or specificity might be preferable. However, the results reveal that instead of using only one set of gamma criteria and a single fail rate threshold, different methods with different gamma criteria and thresholds are needed to optimally detect different types of relevant errors. As was clear from previous work as well ${ }^{26-28}$, the most commonly used $(3 \%, 3 \mathrm{~mm})$ criteria are not sensitive enough to detect many significant anatomical changes (Table 4.3). Using these criteria and a $10 \%$ gamma fail rate threshold does not optimize the trade-off between sensitivity and specificity.

In clinical practice, more uncertainties will be introduced than in this simulation study. Breathing motion, patient positioning, timing and setup are all factors that influence the ability of the portal dosimetry methods to detect relevant anatomical changes. Although, for instance, patient setup errors will be minimized before dose delivery by means of image guided radiotherapy, the gamma fail rate thresholds used in clinical practice likely need to be higher to account for these uncertainties. For the TR gamma analysis, the optimal time-to-agreement and time of interest ${ }^{6,24}$ need to be determined as well in a clinical setting, as in reality the synchronization in time will not be perfect.

An additional difficulty is that more than one anatomical change can occur simultaneously, e.g., pleural effusion may cause a tumor shift ${ }^{18}$, and these may not be easily separable based on gamma analysis results. Furthermore, other anatomical changes can appear in lung cancer patients. Tumor progression is one example, although occurring infrequently ${ }^{35}$ and therefore not considered in this work. Atelectasis is seen more often, but cannot be simulated consistently due to its unpredictable nature. As atelectasis is likely to cause tumor shift ${ }^{11}$, it might be possible to detect this indirect anatomical change instead.

Other methods for evaluating anatomical changes exist, such as visual inspection of CBCT images or tumor tracking ${ }^{36,37}$. However, these do not allow direct evaluation of the dose deviations caused by anatomical changes. Dose recalculation 
based on CBCT images does allow assessment of dose changes due to changing anatomy, but requires daily CBCT imaging. As was shown, large amounts of pleural effusion, which might be a cause for plan adaptation solely based on visual inspection, do not necessarily cause large deviations in the dose distribution. Hence, it is important to combine in-room imaging techniques with portal dosimetry, for instance by recalculating the $3 \mathrm{D}$ dose (based on PDIs) on CBCT images. This can also aid in determining which anatomical change occurred.

\section{Conclusion}

This simulation study investigated the ability of 2D-TI, 2D-TR and 3D-TI portal dosimetry to detect dosimetric consequences of anatomical changes in lung cancer patients. This work confirms that transit portal dosimetry can be used to detect (simulated) anatomical changes in lung cancer patients, provided they are of a certain magnitude. The correlation between the gamma fail rate and DVH metrics at the patient level can be useful for creating decision protocols for adaptive radiotherapy. It may be possible by individual simulation to establish a relationship between DVH metrics and gamma fail rates before treatment. This was not possible before, as on the group level there is no correlation. Error-type specific optimal combinations of a portal dosimetry method, gamma criteria and gamma fail rate threshold should be used to determine if an anatomical change occurred and whether it is clinically relevant. In future work, this research should be extended to include, for instance, different tumor sites, DVH metrics, dose to OARs and 3D-TR portal dosimetry, which may be able to detect changes that are hidden by the 3D-TI method due to the integration. Furthermore, the uncertainties of the portal dosimetry methods need to be quantified before clinical decision models can be based on their outcomes. To conclude, this work shows that clinical decision models for lung cancer patients should be developed on an individual basis, and that a one-size-fits-all approach with respect to portal dosimetry method, gamma criteria and gamma fail rate is not suitable for detecting different types of anatomical changes.

\section{Acknowledgments}

Varian Medical Systems provided the funding for this study (Project:TimePort-III). 


\section{References}

1. Bortfeld T. IMRT: A review and preview. Physics in Medicine and Biology, 2006. 51(13): p. R36379.

2. Teoh M, Clark C, Wood K, Whitaker S, and Nisbet A. Volumetric modulated arc therapy: A review of current literature and clinical use in practice. British Journal of Radiology, 2011. 84: p. 967-96.

3. van Elmpt W, McDermott L, Nijsten S, Wendling M, Lambin P, and Mijnheer B. A literature review of electronic portal imaging for radiotherapy dosimetry. Radiotherapy and Oncology, 2008. 88(3): p. 289-309.

4. Liu B, Adamson J, Rodrigues A, Zhou F, Yin F-f, and Wu Q. A novel technique for VMAT QA with EPID in cine mode on a Varian TrueBeam linac. Physics in Medicine and Biology, 2013. 58(19): p. 6683-700.

5. Woodruff HC, Fuangrod T, Rowshanfarzad P, McCurdy BMC, and Greer PB. Gantry-angle resolved VMAT pretreatment verification using EPID image prediction. Medical Physics, 2013. 40(8): p. 081715.

6. Podesta M, Nijsten SMJJG, Persoon LCGG, Scheib SG, Baltes C, and Verhaegen F. Time dependent pre-treatment EPID dosimetry for standard and FFF VMAT. Physics in Medicine and Biology, 2014. 59(16): p. 4749-68.

7. Spreeuw H, Rozendaal R, Olaciregui-Ruiz I, Gonzalez P, Mans A, Mijnheer B, and van Herk M. Online 3D EPID-based dose verification: Proof of concept. Medical Physics, 2016. 43(7): p. 3969-74.

8. Nijsten SMJJG, Mijnheer BJ, Dekker ALAJ, Lambin P, and Minken AWH. Routine individualised patient dosimetry using electronic portal imaging devices. Radiotherapy and Oncology, 2007. 83(1): p. 65-75.

9. Mans A, Wendling M, McDermott LN, Sonke J-J, Tielenburg R, Vijlbrief R, Mijnheer B, van Herk M, and Stroom JC. Catching errors with in vivo EPID dosimetry. Medical Physics, 2010. 37(6): p. 2638-44.

10. Persoon LCGG, Nijsten SMJJG, Wilbrink FJ, Podesta M, Snaith JAD, Lustberg T, van Elmpt WJC, van Gils F, and Verhaegen F. Interfractional trend analysis of dose differences based on 2D transit portal dosimetry. Physics in Medicine and Biology, 2012. 57(20): p. 6445-58.

11. Persoon LCGG, Egelmeer AGTM, Öllers MC, Nijsten SMJJG, Troost EGC, and Verhaegen F. First clinical results of adaptive radiotherapy based on 3D portal dosimetry for lung cancer patients with atelectasis treated with volumetric-modulated arc therapy (VMAT). Acta Oncologica, 2013. 52(7): p. 1484-89.

12. Persoon LCGG, Podesta M, Nijsten SMJJG, Troost EGC, and Verhaegen F. Time-resolved versus integrated transit planar dosimetry for volumetric modulated arc therapy: Patient-specific dose differences during treatment, a proof of principle. Technology in Cancer Research \& Treatment, 2015. 15(6): p. NP79-87.

13. Yan D, Vicini F, Wong J, and Martinez A. Adaptive radiation therapy. Physics in Medicine and Biology, 1997. 42(1): p. 123-32.

14. McDermott LN, Wendling M, Sonke J-J, van Herk M, and Mijnheer BJ. Anatomy changes in radiotherapy detected using portal imaging. Radiotherapy and Oncology, 2006. 79(2): p. 211-17.

15. Bedford JL, Hanson IM, and Hansen VN. Portal dosimetry for VMAT using integrated images obtained during treatment. Medical Physics, 2014. 41(2): p. 021725.

16. Britton KR, Starkschall G, Tucker SL, Pan T, Nelson C, Chang JY, Cox JD, Mohan R, and Komaki R. Assessment of gross tumor volume regression and motion changes during radiotherapy for Non-Small-Cell Lung Cancer as measured by four-dimensional computed tomography. International Journal of Radiation Oncology • Biology • Physics, 2007. 68(4): p. 1036-46.

17. Kwint M, Conijn S, Schaake E, Knegjens J, Rossi M, Remeijer P, Sonke J-J, and Belderbos J. Intra thoracic anatomical changes in lung cancer patients during the course of radiotherapy. 
Radiotherapy and Oncology, 2014. 113(3): p. 392-397.

18. Møller DS, Khalil AA, Knap MM, and Hoffmann L. Adaptive radiotherapy of lung cancer patients with pleural effusion or atelectasis. Radiotherapy and Oncology, 2014. 110(3): p. $517-22$.

19. Schyns LEJR, Persoon LCGG, Podesta M, van Elmpt WJC, and Verhaegen F. Time-resolved versus time-integrated portal dosimetry: the role of an object's position with respect to the isocenter in volumetric modulated arc therapy. Physics in Medicine and Biology, 2016. 61(10): p. 3969-84.

20. van Elmpt WJC, Nijsten SMJJG, Mijnheer BJ, and Minken AWH. Experimental verification of a portal dose prediction model. Medical Physics, 2005. 32(9): p. 2805-18.

21. van Elmpt W, Nijsten SMJJG, Schiffeleers RFH, Dekker ALAJ, Mijnheer BJ, Lambin P, and Minken AWH. A Monte Carlo based three-dimensional dose reconstruction method derived from portal dose images. Medical Physics, 2006. 33(7): p. 2426-34.

22. Low DA, Harms WB, Mutic $S$, and Purdy JA. A technique for the quantitative evaluation of dose distributions. Medical Physics, 1998. 25(5): p. 656-61.

23. Low DA and Dempsey JF. Evaluation of the gamma dose distribution comparison method. Medical Physics, 2003. 30(9): p. 2455-64.

24. Podesta M, Persoon LCGG, and Verhaegen F. A novel time dependent gamma evaluation function for dynamic 2D and 3D dose distributions. Physics in Medicine and Biology, 2014. 59(20): p. 5973-85.

25. Low DA, Morele D, Chow P, Dou TH, and Ju T. Does the $\gamma$ dose distribution comparison technique default to the distance to agreement test in clinical dose distributions? Medical Physics, 2013. 40(7): p. 071722.

26. Yan G, Liu C, Simon TA, Peng LC, Fox C, and Li JG. On the sensitivity of patient-specific IMRT QA to MLC positioning errors. Journal of Applied Clinical Medical Physics, 2009. 10(1): p. 120-28.

27. Stasi M, Bresciani S, Miranti A, Maggio A, Sapino V, and Gabriele P. Pretreatment patientspecific IMRT quality assurance: A correlation study between gamma index and patient clinical dose volume histogram. Medical Physics, 2012. 39(12): p. 7626-34.

28. Heilemann G, Poppe B, and Laub W. On the sensitivity of common gamma-index evaluation methods to MLC misalignments in Rapidarc quality assurance. Medical Physics, 2013. 40(3): p. 031702.

29. Fraass B, Doppke K, Hunt M, Kutcher G, Starkschall G, Stern R, and Van Dyke J. American Association of Physicists in Medicine Radiation Therapy Committee Task Group 53: Quality assurance for clinical radiotherapy treatment planning. Medical physics, 1998. 25(10): p. 1773-1829.

30. Zou KH, Tuncali K, and Silverman SG. Correlation and simple linear regression. Radiology, 2003. 227(3): p. 617-22.

31. Kumar R and Indrayan A. Receiver operating characteristic (ROC) curve for medical researchers. Indian Pediatrics, 2011. 48(4): p. 277-87.

32. Brahme A. Dosimetric precision requirements in radiation therapy. Acta Radiologica: Oncology, 1984. 23(5): p. 379-91.

33. Waghorn BJ, Meeks SL, and Langen KM. Analyzing the impact of intrafraction motion: Correlation of different dose metrics with changes in target D95\%. Medical Physics, 2011. 38(8): p. 4505-11.

34. Persoon LCGG, Podesta M, Hoffmann L, Sanizadeh A, Schyns LEJR, de Ruiter BM, Nijsten SMJJG, Muren LP, Troost EGC, and Verhaegen F. Is integrated transit planar portal dosimetry able to detect geometric changes in lung cancer patients treated with volumetric modulated arc therapy? Acta Oncologica, 2015. 54(9): p. 1501-07.

35. van Zwienen M, van Beek S, Belderbos J, van Kranen S, Rasch C, van Herk M, and Sonke J. Anatomical changes during radiotherapy of lung cancer patients. International Journal of 
Radiation Oncology • Biology • Physics, 2008. 72(1): p. S111.

36. Shimizu S, Shirato H, Ogura S, Akita-Dosaka H, Kitamura K, Nishioka T, Kagei K, Nishimura $\mathrm{M}$, and Miyasaka K. Detection of lung tumor movement in real-time tumor-tracking radiotherapy. International Journal of Radiation Oncology • Biology • Physics, 2001. 51(2): p. 304-10.

37. Belderbos J, van Beek S, van Kranen S, Rasch C, van Herk M, and Sonke J. Anatomical changes during radiotherapy of lung cancer patients. International Journal of Radiation Oncology • Biology • Physics, 2007. 69(3): p. S508-09. 


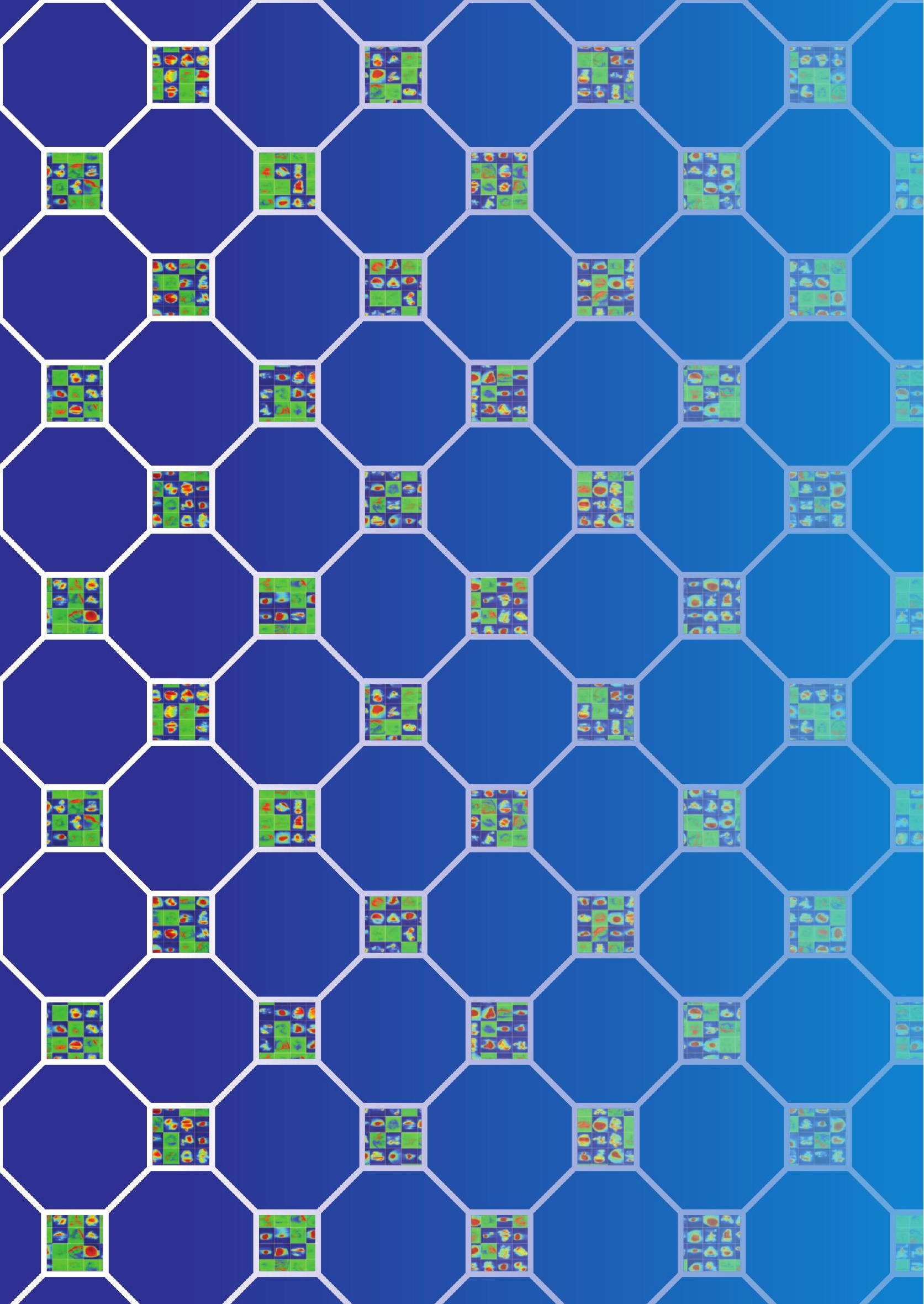




\section{Part II}

\section{Advanced methods for error}

classification using portal dosimetry 



\section{Chapter 5}

\section{Deep learning for error detection in EPID dosimetry: A proof of concept}

Authors

Cecile J.A. Wolfs, Brent van der Heyden and Frank Verhaegen

Adapted from

International Conference on the use of Computers in Radiation Therapy (ICCR) Conference Proceedings. 2019. Montréal, QC, Canada 


\begin{abstract}
Portal dose images (PDIs) acquired using the electronic portal imaging device (EPID), are used to detect dose deviations during radiotherapy treatment. Objective automatic models to flag relevant errors in EPID-based doses are still lacking, providing potential for artificial intelligence. Gamma maps are often reduced to a few numbers, losing potentially important information. This work presents a deep learning method for flagging relevant dose errors using gamma maps based on EPID dosimetry simulations.

Simulated PDIs were used as input for a convolutional neural network (CNN). Anatomical changes (tumor shift/tumor regression/pleural effusion) were introduced into CT images of 6 lung cancer patients. PDIs were simulated using the original treatment plan. PDIs of modified CTs were compared with the PDI of the original CT, by gamma analysis. Of the 372 resulting gamma maps, 248 maps (4 patients) were used for training, 60 maps ( 1 patient) for validation during training and 64 maps ( 1 patient) for testing. The gold standard classification was DVH-based. The CNN classification was compared with the clinical classification, where gamma maps are flagged if the gamma fail rate (GFR) exceeds $10 \%$. The CNN consisted of 2 convolutional/pooling layers followed by fully connected and Softmax layers. The model was trained for 100 epochs using the Adam optimizer, with learning rate 0.0001 and dropout probability 0.2 to prevent overfitting.

The CNN achieved test accuracy, area under the curve (AUC), sensitivity and specificity of $93.8 \%, 0.88,78.6 \%$ and $98.0 \%$, respectively. The clinical GFR classification was not able to flag any errors in the test set.

Deep learning provides a promising, novel way of analyzing EPID dosimetry results for detection of dosimetric errors during radiotherapy treatment. This is a step forward towards automatic objective decision models for determining which patients may need treatment plan adaptation. This work is being extended to real patient data.
\end{abstract}




\section{Introduction}

Anatomical changes during the course of radiotherapy treatment may lead to deviations in delivered dose, potentially necessitating treatment plan adaptation. Using the electronic portal imaging device (EPID) to obtain portal dose images (PDIs) during treatment, dose deviations can be detected. However, objective automatic decision models to flag relevant errors in EPID-based doses are still lacking. Differences observed in dose comparisons with gamma analysis remain difficult to interpret clinically, and 2D gamma maps are often reduced to just a few numbers, for which thresholds are set based on clinical experience. By using artificial intelligence (AI), the vast amount of complex data generated by EPID dosimetry can be processed and interpreted more objectively and efficiently. This may even lead to additional information not available through gamma metrics, such as which type of error has occurred. As a proof of concept, this work presents a deep learning method for flagging relevant dose errors using gamma maps based on EPID dosimetry simulations.

\section{Materials \& Methods}

Simulated PDIs ${ }^{1}$ were used to train and test a convolutional neural network (CNN). In this dataset, different levels of anatomical changes (tumor shift/tumor regression/ pleural effusion) were introduced into CT images of 6 lung cancer patients. For both the original and modified CTs, PDIs were simulated using the original treatment plan. For each patient, the PDIs based on the modified CTs were compared with the PDI based on the original $\mathrm{CT}$, by $(3 \%, 3 \mathrm{~mm})$ gamma analysis. This resulted in 372 gamma maps in total, of which 248 maps (4 patients) were used for training, 60 maps (1 patient) for validation during training and hyperparameter optimization, and 64 maps (1 patient) for testing.

The gamma maps were divided in two classes: 'error' and 'no error', based on a gold standard dose volume histogram (DVH) classification. 3D dose distributions were calculated for all original and modified CTs using the original treatment plan. If the $\Delta D_{95 \%}$ of the GTV between the original and modified cases was larger than $4 \%$, those anatomical changes and their corresponding gamma maps were classified into the 'error' category. Otherwise, they were classified as 'no error'. Furthermore, the CNN classification ('error'/'no error') was compared with the current clinical classification, where gamma maps are flagged if the gamma fail rate (i.e., proportion of pixels with $|\gamma|>1$ ) inside the treatment field for a (3\%, $3 \mathrm{~mm}$ ) gamma analysis exceeds $10 \%$.

The CNN (Figure 5.1) was trained and tested using the TensorFlow package ${ }^{2}$. It consists of 2 consecutive convolutional/max pooling layers, followed by a fully connected layer and a softmax layer. The rectified linear unit (ReLU) is used as activation function, and dropout regularization is applied to prevent overfitting. After a grid search for the optimal hyperparameters (number of epochs/learning rate/dropout probability), the model was trained for 100 epochs using the Adam optimizer ${ }^{3}$, with learning rate 0.0001 and dropout probability 0.2 . 


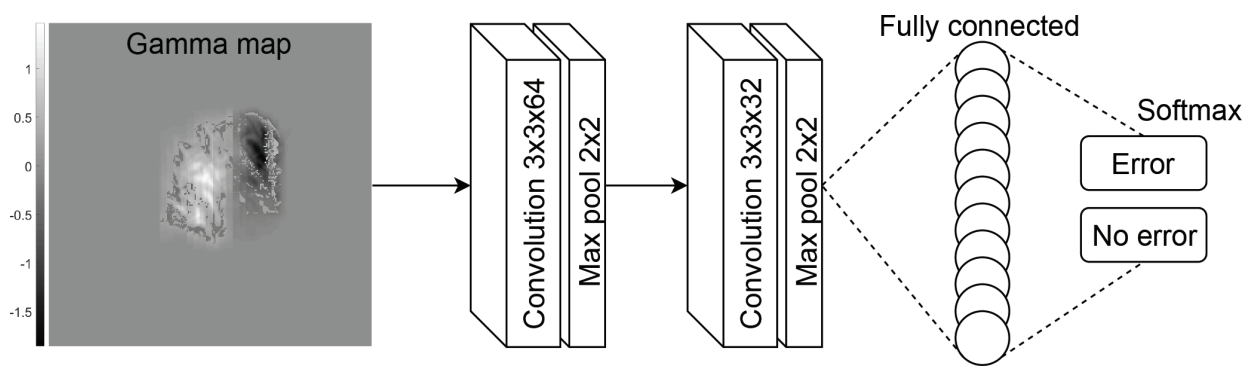

Figure 5.1: Schematic overview of the CNN architecture used in this work. Filter size $\mathrm{x}$ filter size $\mathrm{x}$ number of filters are displayed for each convolutional layer. The fully connected layer consists of 64 nodes.

\section{Results}

The CNN achieved training/validation/test accuracy of $98.8 / 90.0 / 93.8 \%$ and area under the curve (AUC) of $0.98 / 0.92 / 0.88$. With sensitivity of $78.6 \%$ and specificity of $98.0 \%$ on the test data, both the number of false negatives and false positives are low ( 3 and 1 , respectively). The current clinical classification based on gamma fail rate did not flag any errors in the test set. With an accuracy of $68.8 \%$ and an AUC of 0.50 this did not perform better than chance.

\section{Discussion \& Conclusions}

This proof of concept shows that it is feasible to use deep learning for analysis and error classification of gamma maps based on EPID dosimetry, and that it can provide an improvement compared with current clinical error detection thresholds. Further optimization of the CNN architecture may improve the results. This work is being extended to real patient data (i.e., gamma maps obtained from comparing EPID measurements made during treatment with the planned dose), which presents different Al challenges, such as class imbalance. Nevertheless, preliminary results look promising.

To conclude, deep learning provides a promising and novel way of analyzing EPID dosimetry results for detection of relevant dosimetric errors during radiotherapy treatment. This is a step forward towards automatic objective decision models for determining which patients may need treatment plan adaptation.

\section{Acknowledgments}

The Titan Xp GPU used for this research was donated by the NVIDIA Corporation. 


\section{References}

1. Wolfs CJA, Bras MG, Schyns L, Nijsten S, van Elmpt W, Scheib SG, Baltes C, Podesta M, and Verhaegen F. Detection of anatomical changes in lung cancer patients with 2D timeintegrated, 2D time-resolved and 3D time-integrated portal dosimetry: A simulation study. Physics in Medicine and Biology, 2017. 62(15): p. 6044-6061.

2. Abadi M, Barham P, Chen J, Chen Z, Davis A, Dean J, Devin M, Ghemawat S, Irving G, and Isard M. TensorFlow: A system for large-scale machine learning. Presented at OSDI. 2016.

3. Kingma DP and Ba J. Adam: A method for stochastic optimization, in 3rd International Conference for Learning Representations. 2015: San Diego, CA, USA. 



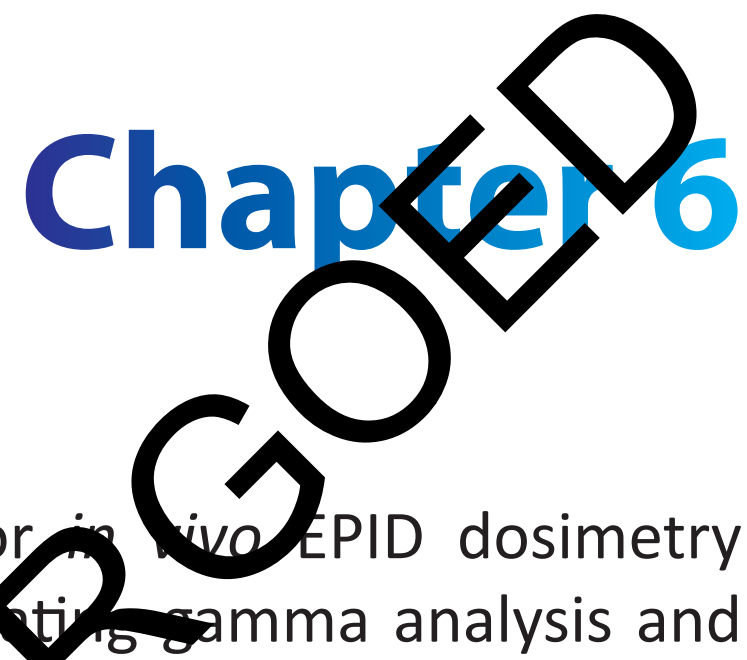
Deep learning for
classification: Re trivamma analysis and 


\section{Chapter 7}

\section{Detection of treatment error types using convolutional neural networks and EPID dosimetry}

Authors

Cecile J.A. Wolfs, Richard A.M. Canters and Frank Verhaegen

Under review 


\begin{abstract}
Electronic portal imaging device (EPID) dosimetry aims to detect treatment errors, potentially leading to treatment adaptation when severe errors occur. Using gamma analysis, measured and predicted EPID dose distributions are compared. Threshold classification methods are used clinically to detect errors, but lead to loss of information as multi-dimensional EPID data is compressed into a few numbers. Advanced classification methods such as deep learning can use all available information. In this study, convolutional neural networks (CNNs) were trained to detect error type and magnitude of simulated treatment errors in lung cancer.

Anatomical changes, positioning errors and mechanical errors were simulated to various extents for lung cancer patients. Predicted portal dose images (PDIs) containing errors were compared to error-free PDIs using gamma analysis. For training of CNNs, 23589 2D gamma maps were used, 6483 for validation/optimization and 6507 as hold-out test set. Three classification levels were assessed: Level 1 (main error type, e.g., anatomical change), Level 2 (error subtype, e.g., tumor regression) and Level 3 (error magnitude/relevance, e.g., >50\% tumor regression).

CNNs showed good performance for all classification levels. Training/test accuracy were $99.5 \% / 96.1 \%$ for Level 1, 92.5\%/86.8\% for Level 2, and 82.0\%/72.9\% for Level 3. For Level 3 classification overfitting became more apparent.

This simulation study indicates that deep learning in the form of CNNs is a promising powerful tool for detecting types and magnitude of clinical treatment errors with EPID dosimetry, providing additional information not currently available from EPID dosimetry in clinical practice.
\end{abstract}




\section{Introduction}

Due to its highly complex nature, in vivo verification of radiotherapy treatment has become increasingly important. An efficient method for performing treatment verification is by using electronic portal imaging device (EPID) dosimetry. The EPID records exit radiation behind the patient during treatment as $2 \mathrm{D}$ images, which are converted to 2D (at the level of the EPID), 3D or 4D doses (in the patient) ${ }^{1-3}$. Measured EPID doses are compared to predicted or planned doses using gamma analysis ${ }^{4,5}$, the standard comparison method for this purpose. The overall aim is detecting various errors potentially occurring during treatment, and adapting treatment when severe, persistent errors occur ${ }^{6}$.

Several studies investigated the sensitivity of EPID dosimetry methods to detect simulated treatment errors and their magnitude ${ }^{7-11}$. However, these usually focus on limited numbers of error types and patients, and often employ simple threshold classification methods based on metrics extracted from gamma maps (e.g., gamma fail/pass rate, mean gamma value). Threshold classification methods are the current clinical standard for analyzing gamma analysis results, but they lead to loss of information, by compressing multi-dimensional EPID data into one or a few numbers. Especially when aiming to detect which type of error has occurred, spatial information can be very valuable, which is lost using threshold methods. However, manually evaluating all EPID data is not possible either, as vast amounts of data are acquired in clinical routine, causing high workloads.

Another approach to processing large amounts of multi-dimensional data has become widely available in recent years, in the form of artificial intelligence (AI). Machine and deep learning techniques have become topics of extensive research in many parts of the radiotherapy chain ${ }^{12}$. Application of these techniques to EPID dosimetry seems especially suitable, as complex data can be input as a whole instead of in reduced form. Particularly deep learning using convolutional neural networks (CNNs) can be useful in this respect ${ }^{13}$. Nyflot et al. ${ }^{14}$ have recently shown the potential of CNNs for extracting features from EPID-based gamma maps to detect errors in multileaf collimator (MLC) positioning for patient specific quality assurance.

To train CNNs accurately, large amounts of labeled data are needed. Although large amounts of data are usually available in radiotherapy clinics, labels are often missing as it is time-consuming to manually evaluate all images. To overcome this issue, in this work a wide range of possible treatment errors was simulated, such that the exact error type and magnitude were known. CNNs were trained to detect which errors occurred, using 2D gamma maps as input. Additionally, the sensitivity of CNNs as an error detection method was evaluated by investigating the error detection limits. E.g., is it possible for CNNs to differentiate between anatomical patient changes and mechanical beam delivery errors, or between tumor shift and regression, or even between tumor shift inside and outside the PTV? This simulation study serves as a first step to evaluate the potential of deep learning applied to in vivo EPID dosimetry for detecting treatment error types.

\section{Materials and Methods}

Error simulation

Data from 47 lung cancer patients (59 treatment plans, 175 unique treatment beams) 
was used in this study. Patients were treated with volumetric modulated arc therapy (VMAT) or a hybrid plan (i.e., consisting of both static beams and VMAT arcs). Using the original treatment plans and $\mathrm{CT}$ images, the transit EPID dose, i.e., the original portal dose image $\left(\mathrm{PDI}_{0}\right)$, was predicted with in-house developed software ${ }^{15}$. In this work, only time-integrated PDIs over treatment beams are considered, i.e., no time-resolved information per beam segment is used.

A wide range of errors was simulated to include a variety of errors that can occur during radiotherapy treatment. These can be grouped into three main types: anatomical changes, positioning errors and mechanical errors. Within the anatomical change category, tumor shift, tumor regression, pleural effusion in the lung, and shift of the mediastinum were simulated. The positioning error category included patient translations and rotations, whereas the mechanical error category comprised monitor unit (MU) scaling errors and MLC shifts. The error magnitude ranges simulated for each treatment plan are listed in Table 7.1.

Table 7.1: Overview of the simulated errors, their ranges and directions. S: superior, I: inferior, L: left, R: right, $\mathrm{A}$ : anterior, P: posterior, GTV: gross tumor volume, MLC: multileaf collimator, $\mathrm{MU}$ : monitor unit, n/a: not applicable.

\begin{tabular}{|c|c|c|c|}
\hline Error type & Error subtype & $\begin{array}{l}\text { Range of error } \\
\text { magnitude } \\
\text { (step size) }\end{array}$ & Direction \\
\hline \multirow{4}{*}{$\begin{array}{l}\text { Anatomical } \\
\text { changes }\end{array}$} & Tumor shift & $0.3-2.4 \mathrm{~cm}(0.3)$ & $\begin{array}{l}\text { If possible, i.e., without moving } \\
\text { GTV out of the lung, S-I and I-S. } \\
\text { Otherwise only S-I or I-S, } \\
\text { depending on location of GTV } \\
\text { in the lung. }\end{array}$ \\
\hline & Tumor regression & $10-90 \%(10)$ & $\mathrm{n} / \mathrm{a}$ \\
\hline & Pleural effusion & $0.5-5 \mathrm{~cm}(0.5)$ & $\mathrm{n} / \mathrm{a}$ \\
\hline & $\begin{array}{l}\text { Mediastinum } \\
\text { shift }\end{array}$ & $0.2-1.6 \mathrm{~cm}(0.2)$ & L-R and R-L \\
\hline \multirow{2}{*}{$\begin{array}{l}\text { Patient } \\
\text { positioning } \\
\text { errors }\end{array}$} & Translation & $0.3-2.1 \mathrm{~cm}(0.3)$ & $\begin{array}{c}\text { L-R and R-L, A-P and P-A, S-I } \\
\text { and I-S }\end{array}$ \\
\hline & Rotation & $0.2-1^{0}(0.2)$ & Around L-R, A-P, S-I axes \\
\hline \multirow{4}{*}{$\begin{array}{l}\text { Linac } \\
\text { mechanical } \\
\text { errors }\end{array}$} & $\begin{array}{l}\text { MLC shift } \\
\text { systematic }\end{array}$ & $0.2-2 \mathrm{~mm}(0.2)$ & $\begin{array}{c}\text { Inwards both banks (closing), } \\
\text { outwards both banks } \\
\text { (opening), in- or outwards one } \\
\text { bank (one-sided) }\end{array}$ \\
\hline & $\begin{array}{l}\text { MLC shift } \\
\text { random }\end{array}$ & $0.2-2 \mathrm{~mm}(0.2)$ & $\begin{array}{l}\text { All leaves random, stuck leaf } \\
\text { (10 simulations each) }\end{array}$ \\
\hline & $\begin{array}{l}\text { MU scaling } \\
\text { systematic }\end{array}$ & $1-10 \%(1)$ & $\mathrm{n} / \mathrm{a}$ \\
\hline & $\begin{array}{l}\text { MU scaling } \\
\text { random }\end{array}$ & $1-10 \%(1)$ & n/a (10 simulations) \\
\hline
\end{tabular}


Anatomical changes and positioning errors were artificially introduced into patient CT images, while for mechanical errors treatment plans were modified. Tumor and mediastinum shift were applied by shifting the voxels of the gross tumor volume (GTV) or mediastinum structure, respectively. For tumor regression, the GTV structure was non-rigidly deformed towards its center, and for pleural effusion a layer of gravitationally pooled fluid (Hounsfield unit value: 0 ) was simulated at the posterior side of the lung containing the GTV. In the case of positioning errors, the complete CT image was translated or rotated. Three types of systematic MLC errors were simulated, by applying shifts to active MLC leaves (i.e., MLC leaves actually shaping the field) for all control points in each treatment beam. The same inward or outward shift was applied to all leaves for opening and closing errors, while for one-sided errors the shift was applied to only one of the MLC leaf banks. The two random MLC errors consisted (i) of applying random shifts to each active MLC leaf and each control point, and (ii) of randomly selecting one active leaf and fixing its position for all following control points (stuck leaf). For the systematic MU errors, one scaling factor was applied to all control points in the treatment beam, while for the random MU errors the scaling factor varied per control point.

The modified CT images or treatment plans were used to predict a new PDI: $\mathrm{PDI}_{\text {error }}$ Comparing $\mathrm{PDI}_{0}$ and $\mathrm{PDI}_{\text {error }}$ per beam was done using gamma analysis, with (3\%, $3 \mathrm{~mm})$ dose difference and distance-to-agreement criteria. The error simulation process resulted in a total of 36579 2D gamma maps. Figure 7.1 shows an example of an original and modified CT image, $\mathrm{PDI}_{0}$ and PDI ${ }_{\text {error }}$ of one of the VMAT arcs of this patient's treatment plan, and the resulting gamma map. For visualization purposes an RGB color map is applied to the gamma image, while in the remainder of this work
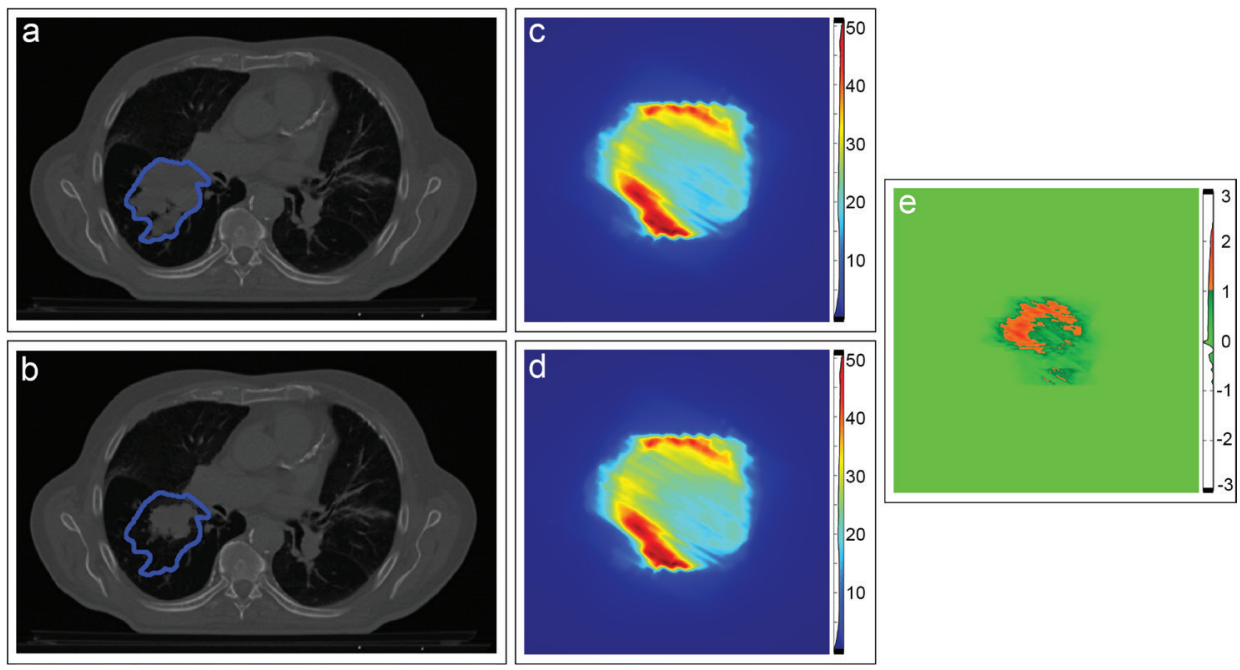

Figure 7.1: Example of simulated tumor regression. a) Axial slice of the original CT image with the GTV delineated in blue. b) Same CT slice as in a), showing simulated tumor regression. The original GTV contour is displayed for comparison. c) PDI, based on a). The color bar shows the dose in cGy. d) PDI error' $^{\prime}$ based on b). The color bar shows the dose in cGy. e) Gamma map of the (3\%, $3 \mathrm{~mm}$ ) comparison between c) and d). As can be derived from the color bar, red areas in the gamma map indicate $\gamma>1$, hence areas of overdosage. Areas of underdosage $(\gamma<-1)$ would appear in blue, but these are not present in this gamma map. 
gamma maps are treated as grayscale images (displayed in Supplementary Material 7A).

\section{Classification model and evaluation}

The gamma maps were used as input for CNNs. For training, validation/optimization and testing of the CNNs, the dataset of gamma maps was split into a training, validation and hold-out test set. While splitting the data, it was ensured that all data from a patient belonged to the same split. Data from 32 patients ( 23589 gamma maps) was used for training, 8 patients (6483 gamma maps) for validation, and 7 patients for final testing (6507 gamma maps). Each gamma map, or image, was assigned three labels associated with different classification levels (Figure 7.2): a Level 1 label (main error type), Level 2 label (error subtype) and Level 3 label (error magnitude). Level 3 classifies relevant versus irrelevant errors by applying a customized threshold on the error magnitude. For each classification level a separate CNN was trained, resulting in three different CNNs. Details about data pre-processing and implementation and optimization of the CNNs are provided in Supplementary Material 7A and 7B.

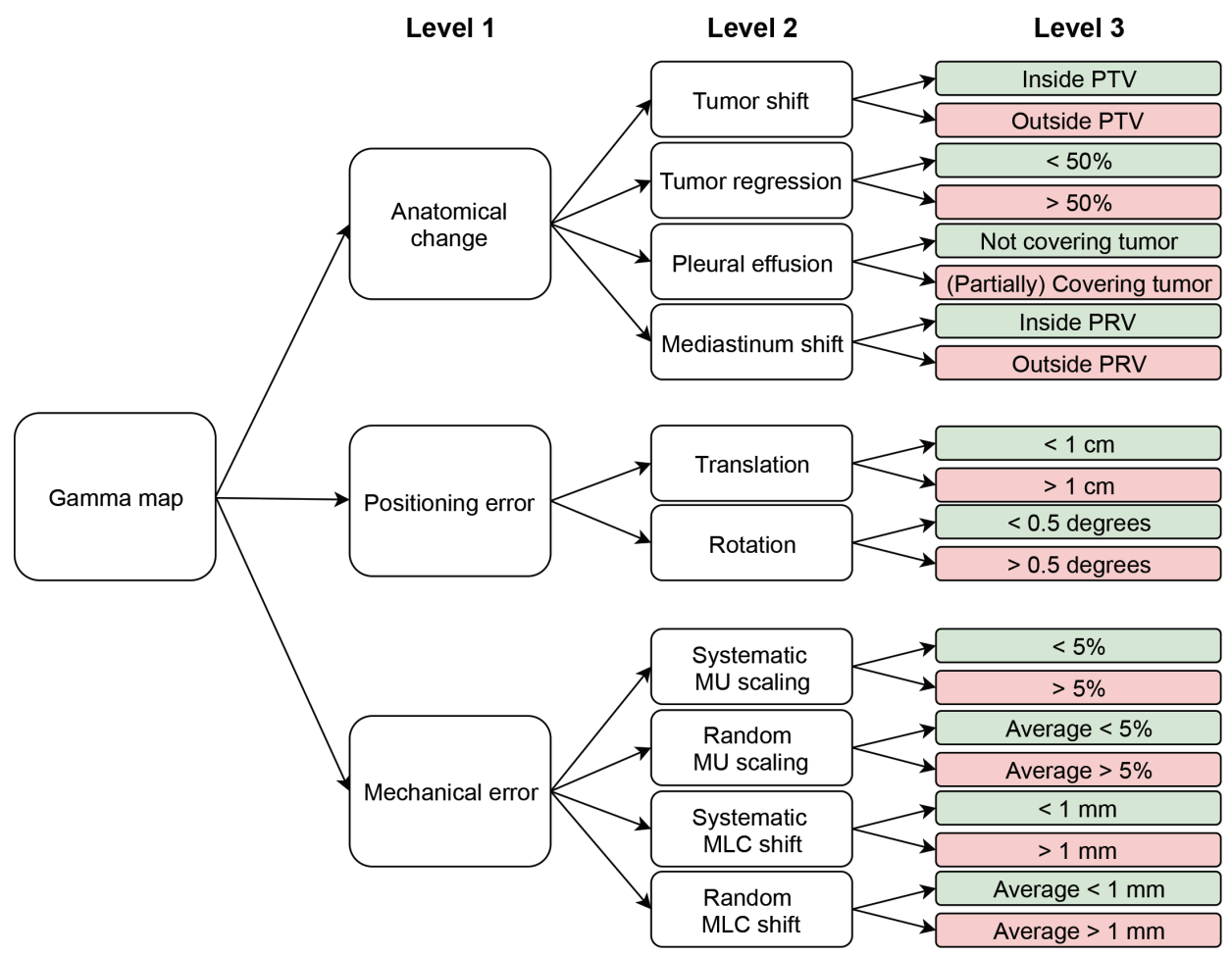

Figure 7.2: Overview of the classification levels and labels/classes per level. For Level 3, the green color indicates classes that are considered irrelevant errors ('no error'), whereas the red color indicates classes that are considered relevant errors ('error'). PTV: planning target volume, PRV: planning organ at risk volume, MU: monitor unit, MLC: multileaf collimator. 
The CNNs' performance was assessed using accuracy and Cohen's kappa ( $\mathrm{K}$ ) as evaluation metrics. Accuracy is defined as the proportion of instances that is classified into the correct class by the model. However, if the data is imbalanced (i.e., the number of images in each class is not roughly equal) accuracy can be influenced heavily by the majority class. $\mathrm{K}$ is a metric that takes class imbalance into account, and therefore gives more unbiased results of model performance. The possible values of $k$ are $[-1,1]$, where $\mathrm{K}$ is 1 for a perfect model and 0 for a model making predictions equal to chance. The confusion matrix, showing true labels versus CNN predicted labels, was examined as well, to determine if there are error types that are misclassified more often than others.

\section{Results}

The results of the CNN optimization are provided in Supplementary Material 7C. In short, the optimal networks for the different classification levels are comparable. Accuracy and $\mathrm{k}$ for training, validation and test sets, obtained with the optimal CNNs, are displayed in Table 7.2. For Levels 1 and 2 training accuracy and $\mathrm{k}$ are higher than 0.9 , hence very good. For Level 3 these are slightly lower, yet still good. This means that CNNs are capable of learning to detect the simulated errors for each classification level. For Levels 1 and 2, performance on the validation and test set is also very good. For Level 3, the differences between training, validation and test performance are slightly larger. This implies that overfitting is more prominent, i.e., the model became too specific to the training data, and less generalizable to data it has not encountered before.

For all levels, confusion matrices for validation and test sets showed similar results, therefore only confusion matrices for training and test sets are shown in Figure 7.3. Considering Level 1 (Figure 7.3a), overall performance is very high, also on the hold-out test set. It is slightly more difficult for the CNN to detect anatomical changes in the test set, compared to the other error types.

In Level 2 (Figure 7.3b), the error subtype misclassified most frequently, in both training and test set, is the random MU error. Additionally, there are error subtypes for which CNN performance is substantially lower for the test set than for the training set, namely mediastinum shift and pleural effusion. The CNN has slightly overfitted for these classes.

Table 7.2: Performance of the CNNs for the different classification levels and datasets

\begin{tabular}{ccccc}
\hline \hline Level & & Training & Validation & Test \\
\hline \hline \multirow{2}{*}{$\mathbf{1}$} & Accuracy (\%) & 99.5 & 98.1 & 96.1 \\
& $\mathbf{K}$ & 0.99 & 0.97 & 0.94 \\
\hline \multirow{2}{*}{$\mathbf{2}$} & Accuracy (\%) & 92.5 & 89.8 & 86.8 \\
& $\mathbf{K}$ & 0.91 & 0.88 & 0.85 \\
\hline \multirow{2}{*}{3} & Accuracy (\%) & 82.0 & 77.3 & 72.9 \\
& $\mathbf{K}$ & 0.81 & 0.76 & 0.71 \\
\hline
\end{tabular}


Level 3 classification is less accurate (Figure 7.3c). The error types causing most misclassifications in the training set are predominantly random MU errors. Overfitting is mostly an issue for the errors in the anatomical change category. Still, 14 out of 20 classes show moderate to good test accuracy of more than $60 \%$. For most error types, the accuracy for the 'error' class (Cat2) is higher than for 'no error' (Cat1). Generally, there are more false positives than false negatives. That is, within an error subtype, there are more images classified as 'error' while the true class is 'no error', than images classified 'no error' while the true class is 'error'.

\section{Discussion}

The results show that CNNs combined with EPID dosimetry are well capable of detecting different types of simulated treatment errors at different classification levels. Training performance is good to very good for all classification levels, however, overfitting becomes apparent for the more detailed classification levels. There are multiple possible explanations for this. On the one hand, more detailed classification levels imply having more labels/classes, thus less images in each class, potentially influencing model performance. Obtaining a larger dataset by simulating errors for additional patients may resolve this. On the other hand, factors in the CNNs, e.g., the number of trainable parameters, can influence overfitting. Further optimization of CNN architecture and using different regularization techniques, e.g., weight regularization, may improve the results.

For Level 2 and 3, it is possible to explain some misclassifications the CNNs make. Due to the use of time-integrated images, random MU errors may average out over VMAT $\operatorname{arcs}^{16,17}$. These are most often misclassified as systematic MU errors, so the CNN is still capable of detecting the error source. Mediastinum shift is most often misclassified as patient translation, potentially due to the mediastinum's size, which is relatively large. As the information in the gamma maps is limited to the radiation field (due to the $10 \%$ low dose threshold), patient translation and mediastinum shift may produce similar patterns in the images. Level 3 misclassifications are mostly within the same error type, causing mainly false positives. These generally add workload, as irrelevant errors are flagged. However, the thresholds separating 'no error' and 'error' in Level 3 can also be optimized, which may lead to different results.

Until now, research concerning CNNs and EPID dosimetry has focused on pretreatment verification and prediction of gamma features from dose distributions s, $18-20^{10}$. In contrast, this study focuses on in vivo treatment errors and classification of error types. Comparing the results of this work is partly possible with those of Nyflot et al. ${ }^{14}$, who developed a model for classifying MLC errors in a pre-treatment setting. Their model achieved $77.8 \%$ accuracy, which is comparable to the average Level 3 accuracy for MLC errors (80.5\%). Comparison to results of the other studies is not possible, as these concern regression models predicting gamma pass rates, which still necessitates threshold-based classification methods and makes classifying error types impossible.

While for conventional threshold classification methods tight gamma criteria, e.g., $(1 \%, 1 \mathrm{~mm})$, may be too sensitive, CNNs might benefit from the additional information in these images. In this work, 1-channel (grayscale) images were used as input, but providing images with an arbitrary number of channels is possible. The different channels could contain gamma maps calculated using different gamma 

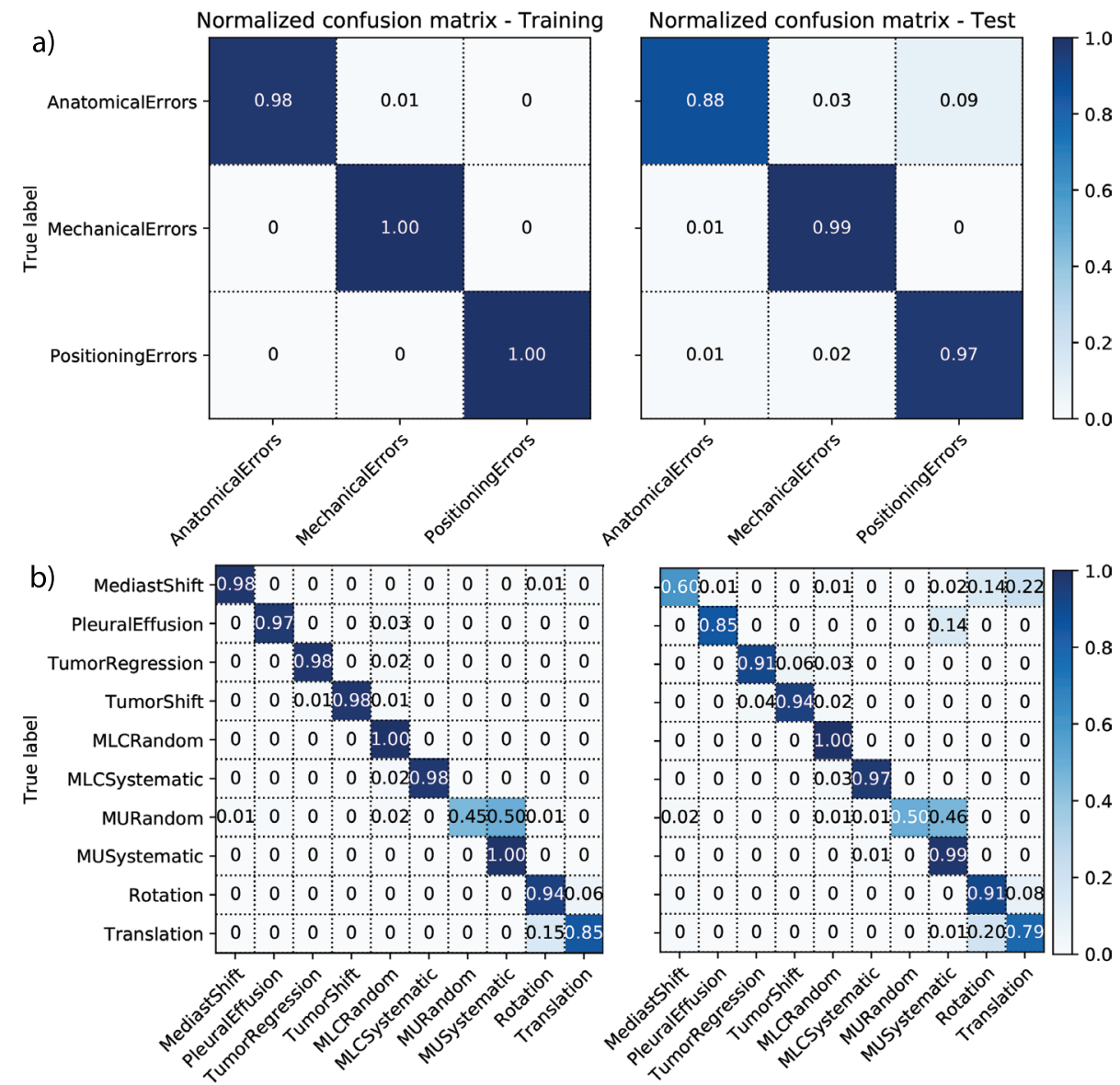

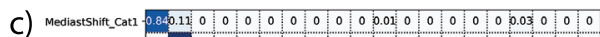

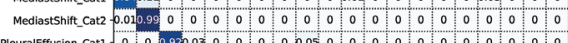

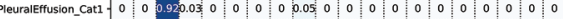

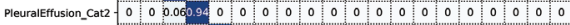

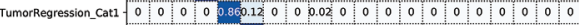
\begin{tabular}{ll|l|l|l|l|l|l|l|l|l|l|l|l|l|l|l|l|l|l} 
TumorRegression_Cat2 & 0 & 0 & 0 & 0 & 0.020 .96 & 0 & 0 & 0.02 & 0 & 0 & 0 & 0 & 0 & 0 & 0 & 0 & 0 & 0 & 0
\end{tabular}

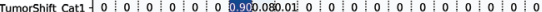

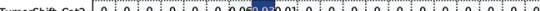

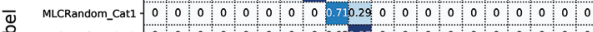

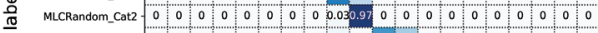

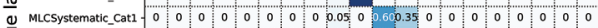

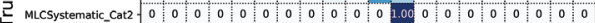

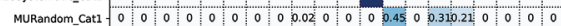

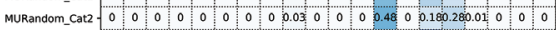

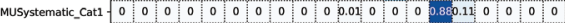

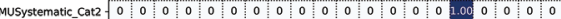
$\begin{array}{lllllllllllllllllllllllllllll}\text { Rotation_Cat1 } & 0 & 0 & 0 & 0 & 0 & 0 & 0 & 0 & 0 & 0 & 0 & 0 & 0 & 0 & 0 & 0 & 0.840 .120 .03 & 0\end{array}$ \begin{tabular}{lllllllllllllllllllllllllll} 
Rotation_Cat2 & 0 & 0 & 0 & 0 & 0 & 0 & 0 & 0 & 0 & 0 & 0 & 0 & 0 & 0 & 0 & 0 & 0.040 .870 .080 .01 \\
\hline
\end{tabular}

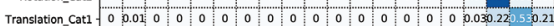

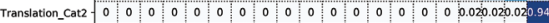

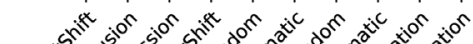
0.0

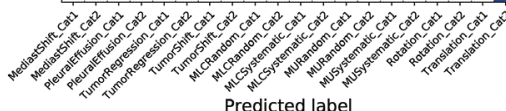

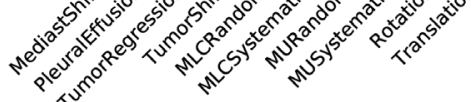

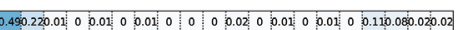

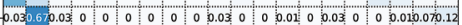

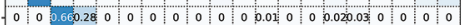

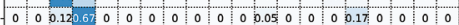

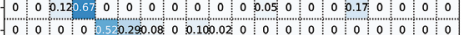

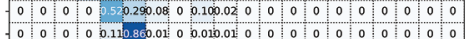

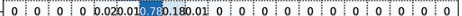

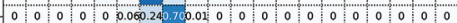

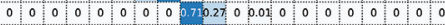

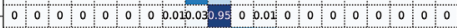

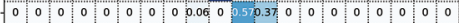

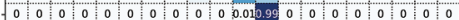

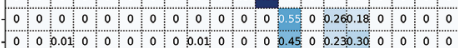

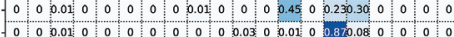

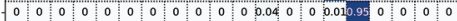

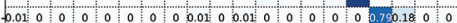

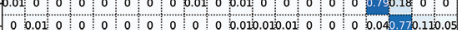

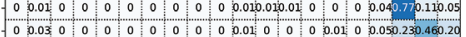

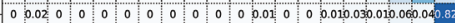

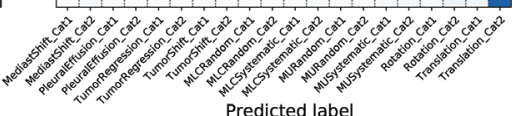

Figure 7.3: Confusion matrices comparing CNN classification (predicted label) with the true label, for the training and test set of a) Level 1, b) Level 2, and c) Level 3 classification. For Level 3 classification, Cat 1 corresponds to the 'no error' class for a certain error type, and Cat 2 to the 'error' class for a certain error type, as displayed in Figure 7.2. 
criteria, so CNNs can learn from combinations of different gamma maps. This can provide more information about, e.g., error magnitude, as small errors may be hidden when using less strict gamma criteria such as $(3 \%, 3 \mathrm{~mm})$. This may especially improve Level 3 classification. Another approach would be to employ different image channels for error-free and error-containing dose images (thus, no gamma maps), such that dose comparison is not performed beforehand, but is incorporated in the CNN.

Naturally, simulated errors are simplifications of real treatment errors. In reality, multiple errors can occur simultaneously. For example, pleural effusion causing tumor shift, or mechanical errors and anatomical changes arising independently from one another. The next step would therefore be to simulate multiple errors simultaneously, and determine if CNNs can still detect the underlying error types, using multi-label classification.

Having the additional information of which error has occurred is beneficial in clinical practice, as different errors have different consequences for patient treatment. For instance, for positioning errors, it is not necessary to make a new treatment plan, while persistent anatomical changes do require re-planning. This information is currently not available from EPID dosimetry with conventional threshold classification methods, and manual inspection of flagged cases is needed. Using the methods described in this work, this process could be automated, which would save time and workload. Level 3 classification additionally provides information on the magnitude of the error, which can be helpful for automatically distinguishing between relevant and irrelevant errors.

However, it remains to be investigated if CNNs trained on simulated error data can classify real treatment error data, due to simplifications made for error simulations and uncertainties in the radiotherapy and EPID dosimetry process. These uncertainties can be large, and influence the detectability of potentially clinically relevant deviations ${ }^{21,22}$. This study serves as a feasibility test for the proposed CNNbased error detection method, in an ideal scenario without uncertainties. As the results are satisfactory, CNNs are promising for improving error detection with EPID dosimetry and should be investigated further.

To be used in clinical practice, ideally the training and CNN optimization process should be repeated with EPID dosimetry data from treatments where errors have actually occurred. Yet, this data is scarce, and generally not labelled. To overcome this, a pre-training approach could be used. CNNs can be trained first on an extensive dataset of simulated errors, and fine-tuned afterwards on a smaller dataset of real treatment errors, using model weights from training on simulated error data as a starting point. This approach would also facilitate transferring models between institutes. Each institute can start from the same pre-trained CNN, and finetune it to their specific data. Another approach is actively labeling all data from EPID measurements for a period of time, so a proper training database can be established. This will however increase workload, although this could be distributed over multiple collaborating institutes, and is likely to result in an imbalanced dataset, as there are many more fractions with no or very small treatment errors than fractions with larger errors. It should be noted that Al models can only classify errors they are trained on. Hence, when developing a model for clinical practice, all relevant errors should be included in the training process. 
Another direction for future research is the use of time-resolved EPID dosimetry. It has been shown previously that time-integrated EPID dosimetry can average out errors in dynamic treatments, that would have been detected with time-resolved EPID dosimetry ${ }^{16,17}$. This effect was also visible for random mechanical errors in Level 2 and 3 classification, where errors were applied to each beam per control point, but potentially averaged out when summing up all control points in the VMAT arcs. Additionally, different errors could be simulated that would benefit from time-resolved dosimetry, e.g., MLC or MU errors only occurring in part of the VMAT arc. It would also allow including more sources of information, e.g., contour projections of relevant structures per control point, which would aid in Level 3 classification. Especially for anatomical change categories related to structures (e.g., pleural effusion covering or not covering the tumor, or tumor shift inside or outside the PTV) this would likely be beneficial.

In conclusion, deep learning in the form of CNNs is a promising powerful tool for detecting types of treatment errors with EPID dosimetry. Errors can be detected to a high level of detail, illustrating the high sensitivity of CNNs as error detection method. This approach can provide additional information not currently available from EPID dosimetry in clinical practice, and has the potential to turn EPID dosimetry into a much more effective and automated method for detecting treatment errors.

\section{Acknowledgments}

This study was funded by Varian Medical Systems (project: Decision DGRT-I). The Titan Xp GPUs used for this research were donated by the NVIDIA Corporation. The authors would like to thank G. van lersel and R. de Vries for their contribution to the mechanical error simulations, Dr. B. van der Heyden for technical support with GPU computing, and Dr. C. Baltes and Dr. S. Scheib from Varian Medical Systems for commenting on the manuscript. 


\section{References}

1. Nijsten SMJJG, van Elmpt WJ, Jacobs M, Mijnheer BJ, Dekker AL, Lambin P, and Minken AW. A global calibration model for a-Si EPIDs used for transit dosimetry. Medical Physics, 2007. 34(10): p. 3872-84.

2. van Elmpt W, Nijsten SMJJG, Schiffeleers RFH, Dekker ALAJ, Mijnheer BJ, Lambin P, and Minken AWH. A Monte Carlo based three-dimensional dose reconstruction method derived from portal dose images. Medical Physics, 2006. 33(7): p. 2426-34.

3. Podesta M, Nijsten SMJJG, Persoon LCGG, Scheib SG, Baltes C, and Verhaegen F. Time dependent pre-treatment EPID dosimetry for standard and FFF VMAT. Physics in Medicine and Biology, 2014. 59(16): p. 4749-68.

4. Low DA and Dempsey JF. Evaluation of the gamma dose distribution comparison method. Medical Physics, 2003. 30(9): p. 2455-64.

5. Low DA, Harms WB, Mutic S, and Purdy JA. A technique for the quantitative evaluation of dose distributions. Medical Physics, 1998. 25(5): p. 656-61.

6. Olaciregui-Ruiz I, Beddar S, Greer P, Jornet N, McCurdy B, Fonseca G, Mijnheer B, and Verhaegen $F$. In vivo dosimetry in external beam photon radiotherapy: Requirements and future directions for research, development and clinical practice. Physics and Imaging in Radiation Oncology, 2020.

7. Wolfs CJA, Bras MG, Schyns L, Nijsten S, van Elmpt W, Scheib SG, Baltes C, Podesta M, and Verhaegen $F$. Detection of anatomical changes in lung cancer patients with 2D timeintegrated, 2D time-resolved and 3D time-integrated portal dosimetry: A simulation study. Physics in Medicine and Biology, 2017. 62(15): p. 6044-6061.

8. Bojechko C and Ford EC. Quantifying the performance of in vivo portal dosimetry in detecting four types of treatment parameter variations. Medical Physics, 2015. 42(12): p. 6912-6918.

9. Olaciregui-Ruiz I, Rozendaal R, Kranen S, Mijnheer B, and Mans A. The effect of the choice of patient model on the performance of in vivo 3D EPID dosimetry to detect variations in patient position and anatomy. Medical Physics, 2019.

10. Mijnheer B, Jomehzadeh A, González P, Olaciregui-Ruiz I, Rozendaal R, Shokrani P, Spreeuw $\mathrm{H}$, Tielenburg $\mathrm{R}$, and Mans A. Error detection during VMAT delivery using EPID-based 3D transit dosimetry. Physica Medica, 2018. 54: p. 137-145.

11. Vieillevigne L, Molinier J, Brun T, and Ferrand R. Gamma index comparison of three VMAT QA systems and evaluation of their sensitivity to delivery errors. Physica Medica, 2015. 31(7): p. $720-725$.

12. Thompson RF, Valdes G, Fuller CD, Carpenter CM, Morin O, Aneja S, Lindsay WD, Aerts HJ, Agrimson $\mathrm{B}$, and Deville Jr C. Artificial intelligence in radiation oncology: A specialty-wide disruptive transformation? Radiotherapy and Oncology, 2018. 129(3): p. 421-426.

13. LeCun Y, Bengio Y, and Hinton G. Deep learning. Nature, 2015. 521: p. 436.

14. Nyflot MJ, Thammasorn P, Wootton LS, Ford EC, and Chaovalitwongse WA. Deep learning for patient-specific quality assurance: Identifying errors in radiotherapy delivery by radiomic analysis of gamma images with convolutional neural networks. Medical Physics, 2019. 46(2): p. 456-464.

15. van Elmpt WJC, Nijsten SMJJG, Mijnheer BJ, and Minken AWH. Experimental verification of a portal dose prediction model. Medical Physics, 2005. 32(9): p. 2805-18.

16. Persoon LCGG, Podesta M, Nijsten SMJJG, Troost EGC, and Verhaegen F. Time-resolved versus integrated transit planar dosimetry for volumetric modulated arc therapy: Patient-specific dose differences during treatment, a proof of principle. Technology in Cancer Research \& Treatment, 2015. 15(6): p. NP79-87.

17. Schyns LEJR, Persoon LCGG, Podesta M, van Elmpt WJC, and Verhaegen F. Time-resolved versus time-integrated portal dosimetry: the role of an object's position with respect to the isocenter in volumetric modulated arc therapy. Physics in Medicine and Biology, 2016. 61(10): p. 3969-84. 
18. Interian Y, Rideout V, Kearney VP, Gennatas E, Morin O, Cheung J, Solberg T, and Valdes G. Deep nets vs expert designed features in medical physics: An IMRT QA case study. Medical Physics, 2018. 45(6): p. 2672-2680.

19. Tomori S, Kadoya N, Takayama Y, Kajikawa T, Shima K, Narazaki K, and Jingu K. A deep learningbased prediction model for gamma evaluation in patient-specific quality assurance. Medical Physics, 2018. 45(9): p. 4055-4065.

20. Valdes G, Chan MF, Lim SB, Scheuermann R, Deasy JO, and Solberg TD. IMRT QA using machine learning: A multi-institutional validation. Journal of Applied Clinical Medical Physics, 2017. 18(5): p. 279-284.

21. Baeza JA, Wolfs CJA, Nijsten SMJJG, and Verhaegen F. Validation and uncertainty analysis of a pre-treatment 2D dose prediction model. Physics in Medicine and Biology, 2018. 63(3): p. 035033.

22. Wolfs CJA, Swinnen ACC, Nijsten SMJJG, and Verhaegen F. Should dose from small fields be limited for dose verification procedures?: uncertainty vs. small field dose in VMAT treatments. Physics in medicine and biology, 2018. 63(20): p. 20 NT01. 


\section{Supplementary Material Chapter 7}

\section{Supplementary Material 7A: Data pre-processing}

The predicted PDI size, and consequently the original gamma map size, was $768 \times 768$ pixels. However, these gamma maps contained many pixels with a gamma value very close to 0 outside of the radiation field (Figure 7A.1a), which can be considered redundant information. Therefore, a $10 \%$ low dose threshold was applied, such that rows and columns in which all pixels contained a dose lower than $10 \%$ of the maximum dose (Figure 7A.1b) were removed from the image. Afterwards, the remaining pixel values were rescaled to the range $[0,1]$ to facilitate faster convergence of the $C N N$, and the images were resized to $128 \times 128$ pixels (Figure 7A.1c).

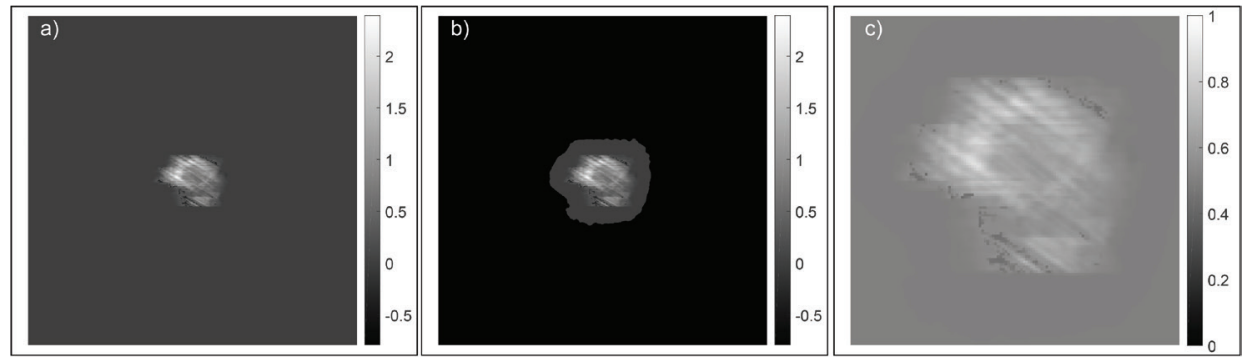

Figure 7A.1: Example of the image preprocessing steps. a) Original gamma map of size $768 \times 768$ pixels. b) Masked gamma map of $768 \times 768$ pixels, showing pixels with a dose lower than $10 \%$ of the maximum dose in black. All rows and columns only containing black pixels will be removed. c) Preprocessed gamma map obtained by removing all-black rows and columns in b), rescaling the remaining pixel values to the range $[0,1]$ and resizing to $128 \times 128$ pixels.

\section{Supplementary Material 7B: Convolutional neural network implementation and optimization}

The CNNs were developed in Keras with the TensorFlow backend ${ }^{1,2}$. The basic CNN architecture (Figure 7B.1) consisted of six blocks of two consecutive convolutional layers and a max pooling layer, followed by one or two fully connected layers and a softmax layer for final classification. The filter size in the convolutional layers was $3 \times 3$; the pooling size in the max pooling layers was $2 \times 2$. Rectified linear unit (ReLU) activation was used in all convolutional and fully connected layers. Categorical cross entropy was used as loss function, and the Adam optimizer was used for optimization of this loss function ${ }^{3}$. The number of filters in each convolutional layer, the number and size of the fully connected layers, the learning rate, dropout rate, and number of epochs were optimized in a hyperparameter search. This optimization process was performed for each of the three classification levels, and therefore resulted in three optimal CNNs. The CNNs were trained using two 12GB Titan Xp GPUs (NVIDIA Corporation, Santa Clara, CA, USA). Data augmentation was performed during the training process by flipping the images horizontally and vertically, as well as by applying random rotations between 0 and $20^{\circ}$. 


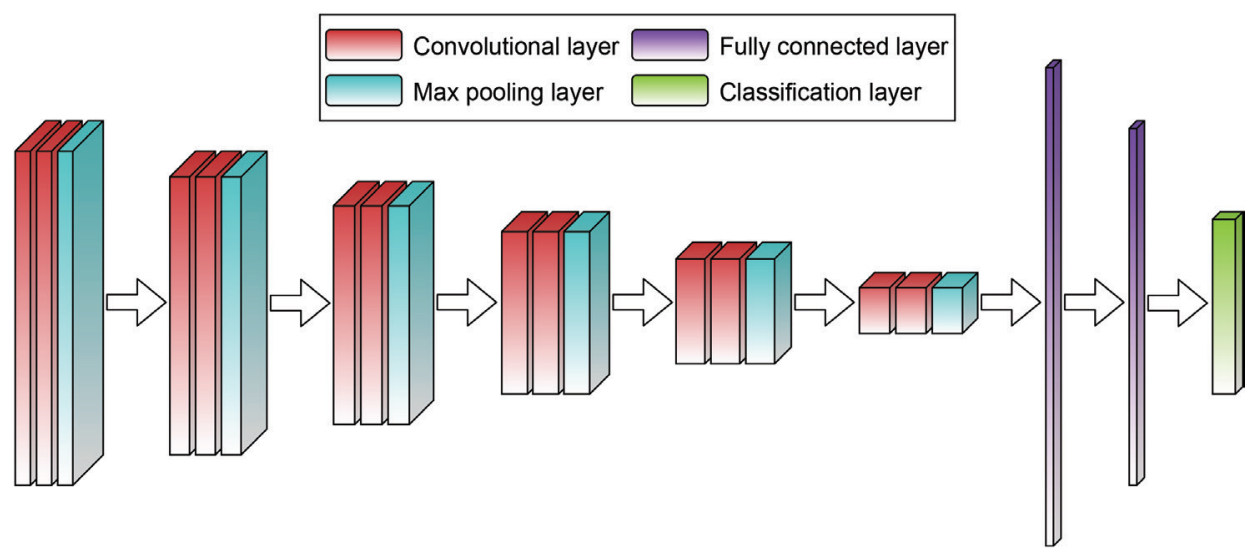

Figure 7B.1: Schematic representation of the CNN architecture.

\section{Supplementary Material 7C: Results of CNN hyperparameter optimization}

The optimal hyperparameters of the CNNs for each classification level are shown in Table 7C.1.To prevent overfitting, the number of trainable parameters was intentionally kept relatively low. The optimal networks for all three classification levels are similar. For Level 3, a smaller number of epochs leads to less overfitting. Additionally, for all levels dropout regularization is beneficial to reduce overfitting. When using dropout regularization, a specified proportion (the dropout rate) of the network units is randomly dropped every training step to prevent dependence between units ${ }^{4}$.

Table 7C.1: Results of the hyperparameter optimization process for the CNNs for each classification level. The number of filters is displayed per block, i.e., the two convolutional layers in the same block of the CNN have the same number of filters.

\begin{tabular}{ccccccc}
\hline \hline Level & $\begin{array}{c}\text { Number of } \\
\text { filters }\end{array}$ & $\begin{array}{c}\text { Fully } \\
\text { connected } \\
\text { layer size }\end{array}$ & $\begin{array}{c}\text { Number of } \\
\text { trainable } \\
\text { parameters }\end{array}$ & $\begin{array}{c}\text { Learning } \\
\text { rate }\end{array}$ & $\begin{array}{c}\text { Number } \\
\text { of } \\
\text { epochs }\end{array}$ & $\begin{array}{c}\text { Dropout } \\
\text { rate }\end{array}$ \\
\hline \hline 1 & $\begin{array}{c}8,8,16,16, \\
32,32\end{array}$ & 256 & 76,139 & $10^{-3}$ & 900 & 0.5 \\
\hline 2 & $\begin{array}{c}8,8,16,16, \\
32,32\end{array}$ & 64,32 & 53,010 & $10^{-3}$ & 1000 & 0.5 \\
\hline 3 & $\begin{array}{c}8,8,16,16, \\
32,32\end{array}$ & 64 & 51,900 & $10^{-3}$ & 600 & 0.5 \\
\hline
\end{tabular}




\section{References Supplementary Material}

1. Chollet F. Keras. 2015; Available from: https://keras.io.

2. Abadi M, Barham P, Chen J, Chen Z, Davis A, Dean J, Devin M, Ghemawat S, Irving G, and Isard M. TensorFlow: A system for large-scale machine learning. Presented at OSDI. 2016.

3. Kingma DP and Ba J. Adam: A method for stochastic optimization, in 3rd International Conference for Learning Representations. 2015: San Diego, CA, USA.

4. Srivastava N, Hinton G, Krizhevsky A, Sutskever I, and Salakhutdinov R. Dropout: A simple way to prevent neural networks from overfitting. The Journal of Machine Learning Research, 2014. 15(1): p. 1929-1958. 




\section{Chapter 8}

External validation of a hidden Markov model for gamma-based classification of anatomical changes in lung cancer patients using EPID dosimetry

Authors

Cecile J.A. Wolfs, Nicolas Varfalvy, Richard A.M. Canters, Sebastiaan M.J.J.G. Nijsten, Djoya Hattu, Louis Archambault* and Frank Verhaegen*

* These authors contributed equally

Adapted from Medical Physics, 2020. Early view publication 


\begin{abstract}
The purpose of this work was to externally validate a hidden Markov model (HMM) for classifying gamma analysis results of in vivo electronic portal imaging device (EPID) measurements into different categories of anatomical change for lung cancer patients. Additionally, the relationship between HMM classification and deviations in dosevolume histogram (DVH) metrics was evaluated.

The HMM was developed at CHU de Québec (CHUQ), and trained on features extracted from gamma analysis maps of in vivo EPID measurements from 483 fractions (24 patients, treated with 3D-CRT or IMRT), using the EPID measurement of the first treatment fraction as reference. The model inputs were the average gamma value, standard deviation, and average value of the highest $1 \%$ of gamma values, all averaged over all beams in a fraction. The HMM classified each fraction into one of three categories: no anatomical change (Category 1), some anatomical change (no clinical action needed, Category 2) and severe anatomical change (clinical action needed, Category 3). The external validation dataset consisted of EPID measurements from 263 fractions of 30 patients treated at Maastro with VMAT or hybrid plans (containing both static beams and VMAT arcs). Gamma analysis features were extracted in the same way as in the CHUQ dataset, by using the EPID measurement of the first fraction as reference $(\gamma Q)$, and additionally by using an EPID dose prediction as reference $(\gamma M)$. For Maastro patients, cone beam CT (CBCT) scans and IGRT classification of these images were available for each fraction. Contours were propagated from the planning $C T$ to the CBCTs, and the dose was recalculated using a Monte Carlo dose engine. DVH metrics for targets and organs-at-risk (OARs: lungs, heart, mediastinum, spinal cord, brachial plexus) were extracted for each fraction, and compared to the planned dose. HMM classification of the external validation set was compared to threshold classification based on the average gamma value alone (a surrogate for clinical classification at CHUQ), IGRT classification as performed at Maastro, and differences in DVH metrics extracted from 3D dose recalculations on the CBCTs.

The HMM achieved 65.4\%/65.0\% accuracy for $\gamma \mathrm{Q}$ and $\gamma \mathrm{M}$, respectively, compared to average gamma threshold classification. When comparing HMM classification with IGRT classification, the overall accuracy was $29.7 \%$ for $\gamma Q$ and $23.2 \%$ for $\mathrm{YM}$. Hence, HMM classification and IGRT classification of anatomical changes did not correspond. However, there is a trend towards higher deviations in DVH metrics with classification into higher categories by the HMM for large OARs (lungs, heart, mediastinum), but not for the targets and small OARs (spinal cord, brachial plexus).

The external validation shows that transferring the HMM for anatomical change classification to a different center is challenging, but can still be valuable. The HMM trained at CHUQ cannot be used directly to classify anatomical changes in the Maastro data. However, it may be possible to use the model in a different capacity, as an indicator for changes in the 3D dose based on 2D EPID measurements.
\end{abstract}




\section{Introduction}

Over the course of radiotherapy treatment, anatomical changes occur frequently in lung cancer patients ${ }^{1-3}$. These changes may cause differences between the planned and delivered radiation dose, potentially leading to underdosing the tumor and/or overdosing the organs-at-risk (OARs) ${ }^{3-5}$. Adaptive radiotherapy strategies can be employed to detect these changes, and to take clinical action if the differences become too large, e.g., by acquiring a new CT scan and adjusting the patient's treatment plan ${ }^{6}$. One method to detect dose deviations due to anatomical changes is by using an electronic portal imaging device (EPID) as a dosimeter during treatment ${ }^{7-10}$. These in vivo EPID measurements can then be compared to a reference dose using gamma analysis ${ }^{11,12}$, to evaluate dose differences and flag patients needing adaptive treatment.

When routinely using the EPID for treatment verification, a vast amount of data is generated, resulting in high workloads if this data needs to be evaluated manually. Furthermore, a relatively small number of patients need adaptive treatment, and unnecessary re-planning should be avoided as it is time-consuming and may increase the overall treatment time ${ }^{13}$. Therefore, automated methods are required for efficient selection of patients potentially needing treatment adaptation and further investigation of the causes of the reported EPID dose deviation. Currently, such methods often consist of fixed thresholds on one variable, such as the gamma fail rate or average gamma value. This may be too simplistic and it remains difficult to relate these methods to clinically used metrics from dose-volume histograms (DVH) $)^{5,14,15}$.

However, with the increased adoption of artificial intelligence (AI) methods in radiation oncology ${ }^{16}$, development of more sophisticated models, which can combine information in a way human observers might not be able to, is becoming more common. In the case of EPID dosimetry, Varfalvy et al. ${ }^{17}$ recently reported using a hidden Markov model (HMM) for classifying different levels of anatomical change in lung cancer patients, based on gamma analysis of in vivo EPID measurements. An HMM is an unsupervised machine learning technique, that is traditionally used for analyzing time series and in speech recognition ${ }^{18}$. The application of this well-established model was the first application of AI techniques to EPID dosimetry.

A pitfall of Al models is that they can become too specific to the dataset they are trained on: they can overfit and thus not perform well when applied to new datasets or in other institutions. Model validation is therefore necessary, to ensure general applicability of the model to new data ${ }^{19}$. Internal validation with a holdout dataset (i.e., part of the data is not included for training the model, but is used to evaluate model performance on data the model has not encountered before) can overcome this problem within an institute. However, external validation with data from different institutes is needed to evaluate the model's generalizability to datasets with differences in acquisition methods and other parameters.

In this study, the HMM developed by Varfalvy et al. ${ }^{17}$ at $\mathrm{CHU}$ de Québec (CHUQ, Québec City, QC, Canada) was externally validated using a dataset from Maastro (Maastricht, the Netherlands). This dataset was inherently different in terms of treatment technique and EPID measurement frequency, among other factors. In addition, the relationship between HMM classification and deviations in DVH metrics was evaluated. 


\section{Materials and Methods}

A detailed explanation of HMMs and their application to anatomical change classification using EPID dosimetry is provided by Varfalvy et al. ${ }^{17}$ In short, each patient can be considered a system that can be in a certain state. In the context of this work, the possible states are different severities of anatomical change. At each time point, or each fraction, a patient remains in the same state or transitions to a different state according to a transition probability matrix. However, these states are not directly observable, i.e., they are hidden. In contrast, the EPID dosimetry results of each fraction are observable, and they form an observation sequence. An emission probability matrix connects the hidden states and observation sequences, by describing the probability of generating a certain observation given that the system is in a certain state. The HMM is defined by these two probability matrices, and the training process consists of finding the most likely probability matrices, based on provided observation sequences.

The HMM developed at CHUQ was trained on features extracted from gamma analysis maps of EPID measurements taken during treatment, using the EPID measurement of the first fraction as a reference, as displayed in Figure 8.1. Specifically, the average gamma value $(\bar{\gamma})$, the standard deviation $\left(\sigma_{\gamma}\right)$ and the average value of the highest $1 \%$ of gamma values $\left(\gamma_{\text {top } 1 \%}\right)$, averaged over all beams in a fraction, were used as input. Based on these features, the HMM classified each fraction into one of three possible categories: no anatomical change (Category 1), some anatomical change (no clinical action needed, Category 2) and severe anatomical change (clinical action needed, Category 3). These categories were based on retrospective clinical evaluation of the data, and each corresponded to a specific range of values (Category $1: \bar{\gamma}<0.4$, Category 2: $0.4 \leq \bar{\gamma}<0.6$, Category $3: \bar{\gamma} \geq 0.6$ ). This facilitated comparison of HMM classification to a simple $\bar{\gamma}$-based threshold classification method, as a surrogate for clinical classification ${ }^{17}$.

The training dataset from CHUQ consisted of daily EPID measurements from 483 fractions of 24 patients treated with 3D conformal radiotherapy (3D-CRT) or intensity modulated radiotherapy (IMRT). Only patients that did not have severe anatomical changes at the start of treatment were included. This was assessed visually

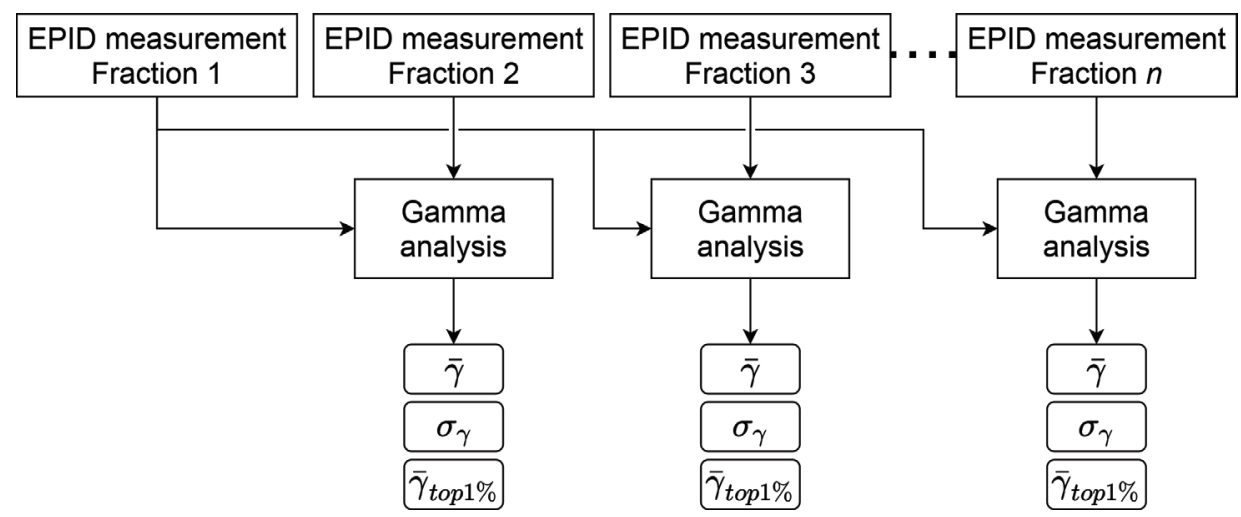

Figure 8.1: Flowchart of the gamma feature extraction process at $C H U Q$, also referred to as $\gamma Q$. $\bar{\gamma}$ : average gamma value, $\sigma_{Y}$ : standard deviation of gamma values, $\gamma_{\text {top } 1 \%}$ : average value of the highest $1 \%$ of gamma values. 
by comparing the planning $\mathrm{CT}(\mathrm{pCT})$ with a cone beam $\mathrm{CT}(\mathrm{CBCT})$ image made before the first fraction. The HMM was internally validated at CHUQ on a similar hold-out dataset of EPID measurements from 559 fractions of 28 additional patients. On this hold-out dataset, the HMM achieved $83 \%$ accuracy compared to the $\bar{\gamma}$-based threshold classification method ${ }^{17}$.

In the present study, the HMM from CHUQ was applied to an external dataset acquired at Maastro. This external validation dataset consisted of EPID measurements from 263 fractions of 30 patients. These patients were treated with volumetric modulated arc therapy (VMAT) or a hybrid plan, the latter comprising both static beams and VMAT arcs. Hence, the treatment modalities differed between the two institutes, making the validation even more pertinent. Time-integrated EPID images were obtained for the first three fractions and every third fraction thereafter, and converted to portal dose images with in-house developed EPID dosimetry software ${ }^{7}$. Gamma analysis ${ }^{11,12}$ was performed in two manners:

1. $\mathrm{yQ}:$ In this scenario, the data was prepared in the same way as the dataset from CHUQ. That is, EPID measurements were compared to the EPID measurement of the first fraction (Figure 8.1$)$, using gamma analysis with $(3 \%, 3 \mathrm{~mm})$ dose difference and distance-to-agreement criteria and a $10 \%$ low dose threshold (i.e., all voxels with dose $<10 \%$ of the maximum dose were ignored).

2. $\gamma M$ : This scenario mimicked Maastro's clinical workflow, in which EPID measurements are compared to an EPID dose prediction ${ }^{20}$ (Figure 8.2), using (5\%, $3 \mathrm{~mm}$ ) gamma analysis. Here, a $10 \%$ low dose threshold was applied as well. EPID dose predictions are calculated using the model developed by van Elmpt et al. ${ }^{20}$ This model is based on pencil beam kernels and predicts the EPID dose behind the patient, using an EPID dose prediction without the patient in the beam, in combination with the radiological thickness of the patient.

The comparison to an EPID dose prediction as well as the use of a different dose difference criterion in the gamma analysis reflect variation in clinical workflow between the two institutes. By comparing EPID measurements to an EPID dose prediction, not only interfractional changes can be detected, but also deviations from the planned dose distribution. Therefore, a slightly larger dose difference criterion is chosen to avoid too many false positive results.

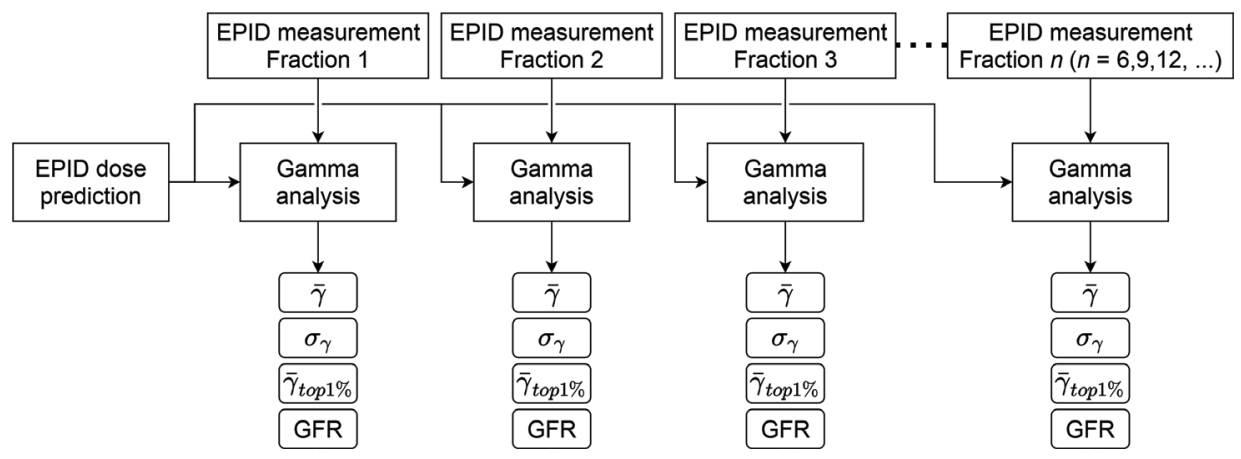

Figure 8.2: Flowchart of the gamma feature extraction process based on Maastro's workflow, also referred to as $\gamma$ M. $\bar{\gamma}$ : average gamma value, $\sigma_{\gamma}$ : standard deviation of gamma values, $\gamma_{\text {top } 1 \%}$ : average value of the highest $1 \%$ of gamma values, GFR: gamma fail rate (proportion of pixels with $|\gamma|>1$ ). 
For both scenarios, $\bar{\gamma}, \sigma_{\gamma}$ and $\gamma_{\text {top } 1 \%}$ were calculated for each beam/arc. Additionally, the gamma fail rate (GFR), i.e., the proportion of pixels with $|\gamma|>1$, was calculated. These features were averaged over all beams in a fraction, as classification of anatomical changes is performed per fraction.

To evaluate the performance of the HMM, the HMM classification of the external validation dataset was compared to the same $\bar{\gamma}$-based threshold classification method used by Varfalvy et al. ${ }^{17}$ as a surrogate for clinical classification. Accuracy is used as an evaluation metric, and is defined as the proportion of fractions that is classified in the same category by both the HMM and the threshold classification method. At Maastro, clinical classification of EPID measurements is based on the GFR, where a GFR $>10 \%$ in the in-field region constitutes a relevant dose difference. Therefore, the distribution of the GFR values in each HMM category was analyzed. The Shapiro-Wilk test ${ }^{21}$ was used to determine whether the GFRs in each category were normally distributed. As they were generally not (results provided in Supplementary Material 8A), Mann-Whitney $U$ tests were performed to determine if the differences in GFR values between each pair of HMM categories were statistically significant.

For the patients in the Maastro dataset, $\mathrm{CBCT}$ images were available for every fraction. These were used to evaluate HMM classification against anatomical change classification according to an image-guided radiotherapy (IGRT) traffic light protocol used at Maastro ${ }^{22}$. Based on visual assessment, CBCT images are classified into one of three categories: Code Green (no anatomical changes), Code Orange (some anatomical changes, no re-planning needed), Code Red (severe anatomical changes, re-planning needed). Although these categories are similar to the categories of anatomical change used at $\mathrm{CHUQ}$, the underlying protocols for classification into these categories are different. The main difference is that at $\mathrm{CHUQ}, \mathrm{CBCT}$ scans and visual assessment of these images are triggered by deviating EPID measurements, hence they are not available for every fraction, while at Maastro CBCT scans and IGRT classification are performed every fraction, regardless of the EPID measurement results. For comparison of HMM and IGRT classification, accuracy was again used as an evaluation metric.

An additional benefit of having CBCT images for every fraction, is that the $3 \mathrm{D}$ dose could be recalculated, and DVH metrics could be extracted. For each CBCT

Table 8.1: DVH metrics evaluated in this study. CTV: clinical target volume, GTV: gross tumor volume.

\begin{tabular}{cc}
\hline \hline Structure & DVH metric \\
\hline \hline Target (primary CTV: CTV1, lymph node CTV: CTV2) & $\mathrm{V}_{95 \%}$ \\
\hline Lungs - GTV & $\mathrm{D}_{\text {mean }}$ \\
& $\mathrm{V}_{5 \mathrm{~Gy}}$ \\
\hline Heart & $\mathrm{D}_{\text {mean }}$ \\
& $\mathrm{D}_{\text {max }}$ \\
\hline Mediastinum & $\mathrm{D}_{\max }$ \\
\hline Spinal cord & $\mathrm{D}_{\max }$ \\
\hline Brachial plexus & $\mathrm{D}_{\max }$ \\
\hline
\end{tabular}


image, contours were propagated from the $\mathrm{pCT}$ to the $\mathrm{CBCT}$ using Mirada Workflow Box (Mirada Medical Ltd., Oxford, UK), and the dose was recalculated using the Monte Carlo dose engine $\mathrm{XVMC}^{23}$. No correction was applied to the Hounsfield units (HUs) of the CBCTs. The DVH metrics extracted and analyzed are listed in Table 8.1; these are also used in the treatment planning process at Maastro as constraints during plan evaluation. The CBCT recalculation process is fully automated, including contour propagation and DVH metric calculation.

To determine if dose deviations observed in gamma analysis corresponded to changes in DVH metrics, the DVH metrics for each CBCT and the $\mathrm{PCT}$ were compared as follows:

$$
\Delta \mathrm{DVH}_{\mathrm{i}}=\left|\mathrm{DVH}\left(\mathrm{CBCT} \mathrm{i}_{\mathrm{i}}\right)-\mathrm{DVH}(\mathrm{pCT})\right|
$$

Where $C B C T_{i}$ is the CBCT image of the $i^{\text {th }}$ fraction, and $D V H$ is one of the relevant DVH metrics (Table 8.1). To ensure that differences in dose were not influenced by using different dose calculation algorithms (Acuros XB (Varian Medical Systems, Palo Alto, ( $A$, USA) vs. XVMC), the dose on the $\mathrm{pCT}$ was first recalculated with the Monte Carlo algorithm as well. Analogous to the GFR analysis, after testing for normality with the Shapiro-Wilk test (results provided in Supplementary Material 8B), Mann-Whitney U tests were applied to evaluate if there were statistically significant differences in $\triangle \mathrm{DVH}$ values between each pair of HMM categories.

\section{Results}

On the Maastro dataset, the model achieved an accuracy of $65.4 \%$ and $65.0 \%$, for $\gamma Q$ and $\gamma \mathrm{M}$ respectively, when comparing with the $\bar{\gamma}$ threshold based classification. As can be seen from the confusion matrices in Figure 8.3, in either scenario the model never misclassified a Category 1 error as Category 3 and vice versa. Fractions that should be in Category 2 according to the threshold classification method are almost always
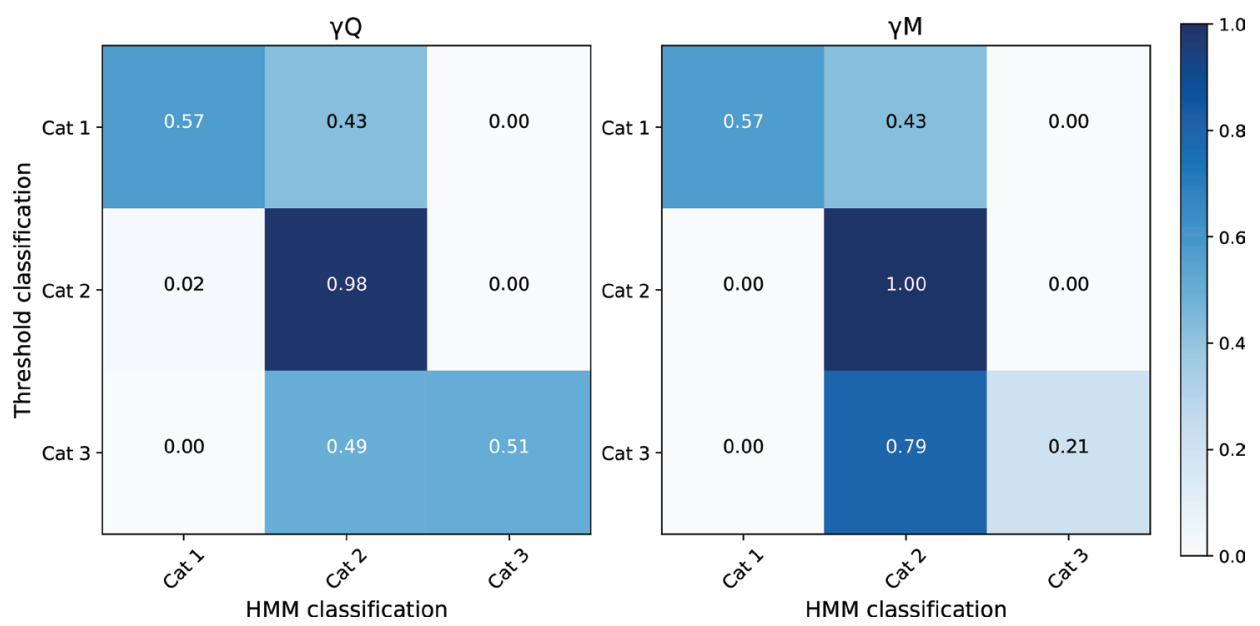

Figure 8.3: Normalized confusion matrices comparing HMM classification to the $\bar{\gamma}$ threshold based classification, for $\gamma \mathrm{Q}$ (left) and $\mathrm{\gamma M}$ (right). The numerical values represent the percentage of fractions in each category along the vertical axis that are classified as belonging to the category listed along the horizontal axes. 
correctly classified by the HMM. However, there are generally too many fractions classified in Category 2. This means that there are false positives (fractions classified in Category 2 that should be in Category 1), which increase workload. There are also false negatives (fractions classified in Category 2 that should be in Category 3), which means that important anatomical changes can be missed. The amount of false negatives is substantially larger ( $49 \%$ vs. $79 \%$, i.e., a $60 \%$ increase) when using $\mathrm{YM}$.

The boxplots in Figure 8.4 show the range of GFR values per category, as classified by the HMM. It can be derived that by using a threshold of $10 \%$ a good distinction between Category 1 and 3 can be made, both for $\gamma \mathrm{Q}$ and $\gamma \mathrm{M}$. There is a statistically significant difference between each pair of categories $(p<0.01)$. Although the range of GFR values in Category 2 overlaps somewhat with both Category 1 and 3 , it would be possible to set two thresholds on the GFR to distinguish between the three categories, e.g., at $5 \%$ and $20 \%$.

When comparing HMM classification with IGRT classification, the overall accuracy was $29.7 \%$ for $\gamma \mathrm{Q}$ and $23.2 \%$ for $\gamma \mathrm{M}$. It is clear from these values, as well as from the confusion matrices in Figure 8.5, that HMM classification and IGRT classification of anatomical changes do not correspond. Slightly more fractions are classified correctly when using $\gamma \mathrm{Q}$ compared to $\gamma \mathrm{M}$.

In Figure 8.6, the $\triangle \mathrm{DVH}$ per metric and per HMM category, excluding outliers, are visualized. The same figure including visualization of outliers is provided in Supplementary Material $8 \mathrm{C}$. Although the ranges of the boxplots overlap, for the lungsGTV, heart and mediastinum, i.e., the large OARs, a trend towards larger differences in DVH metrics with classification in higher category by the HMM can be seen. This trend is statistically significant in most cases $(*: p<0.05 ; * *: p<0.01)$. The $\Delta \mathrm{DVH}$ values of the CTVs and small OARs (spinal cord and brachial plexus) generally do not increase with HMM classification in a higher category. Both $\gamma \mathrm{Q}$ and $\mathrm{\gamma M}$ show the same trends.
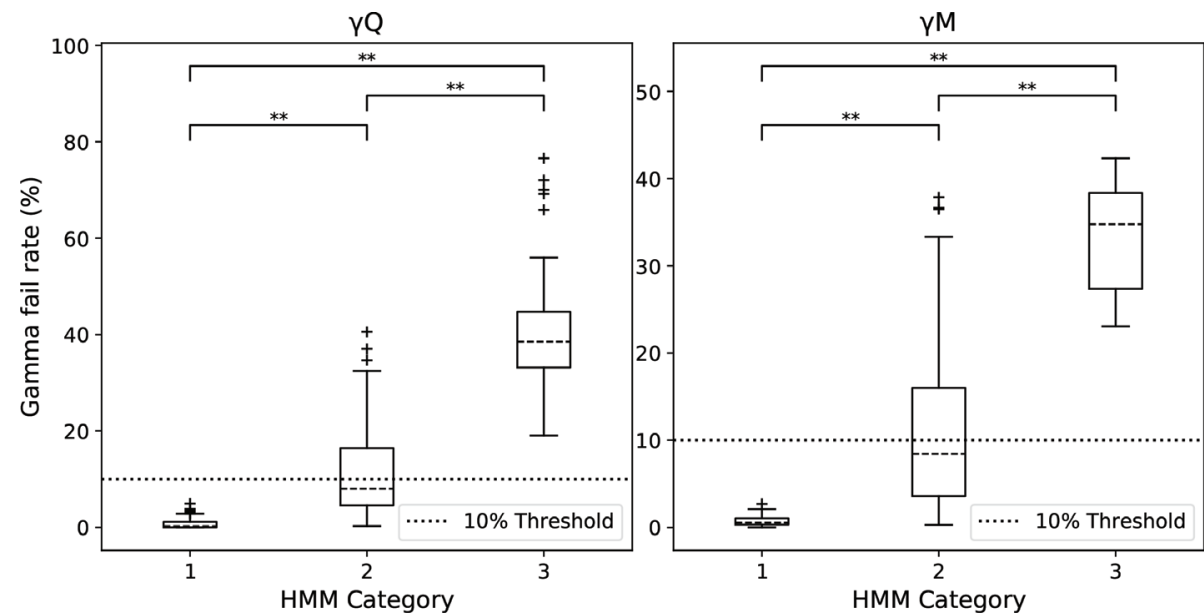

Figure 8.4: Boxplots showing the ranges of gamma fail rates for the different categories of anatomical change, as classified by the HMM. $x$-axis: HMM classification, $y$-axis: gamma fail rate, ${ }^{* *}: p<0.01$. 

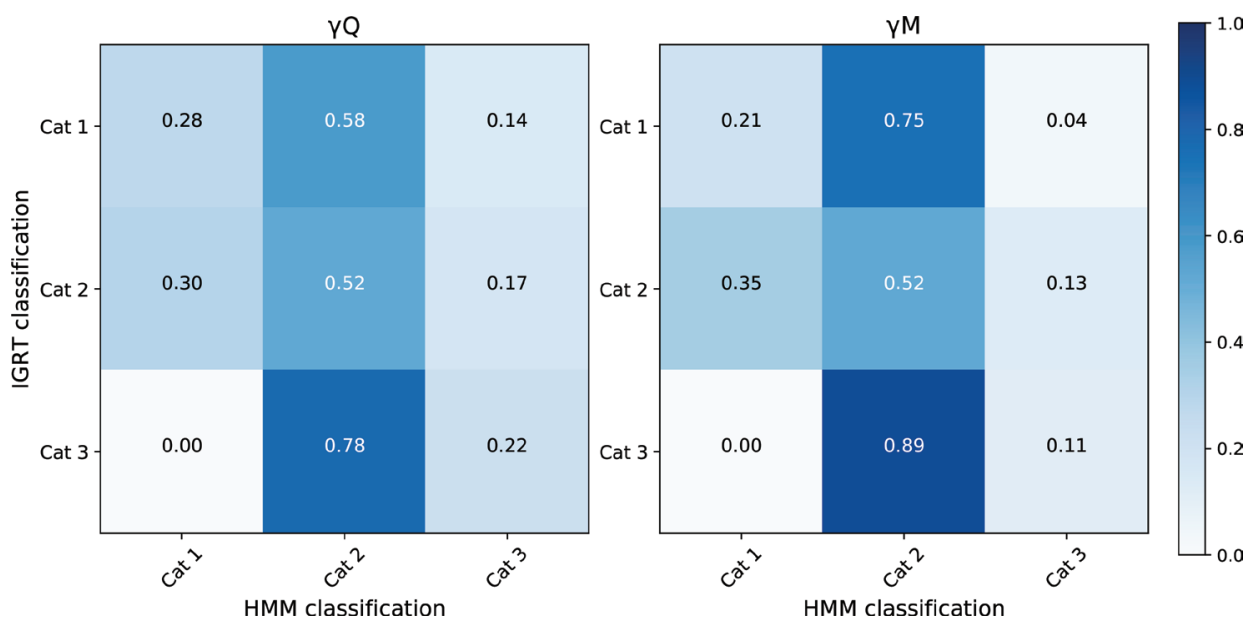

Figure 8.5: Normalized confusion matrices comparing HMM classification to Maastro's IGRT classification, for YQ (left) and YM (right). For the IGRT classification, Cat 1: Code Green, Cat 2: Code Orange, Cat 3: Code Red. The numerical values represent the percentage of fractions in each category along the vertical axis that are classified as belonging to the category listed along the horizontal axes.
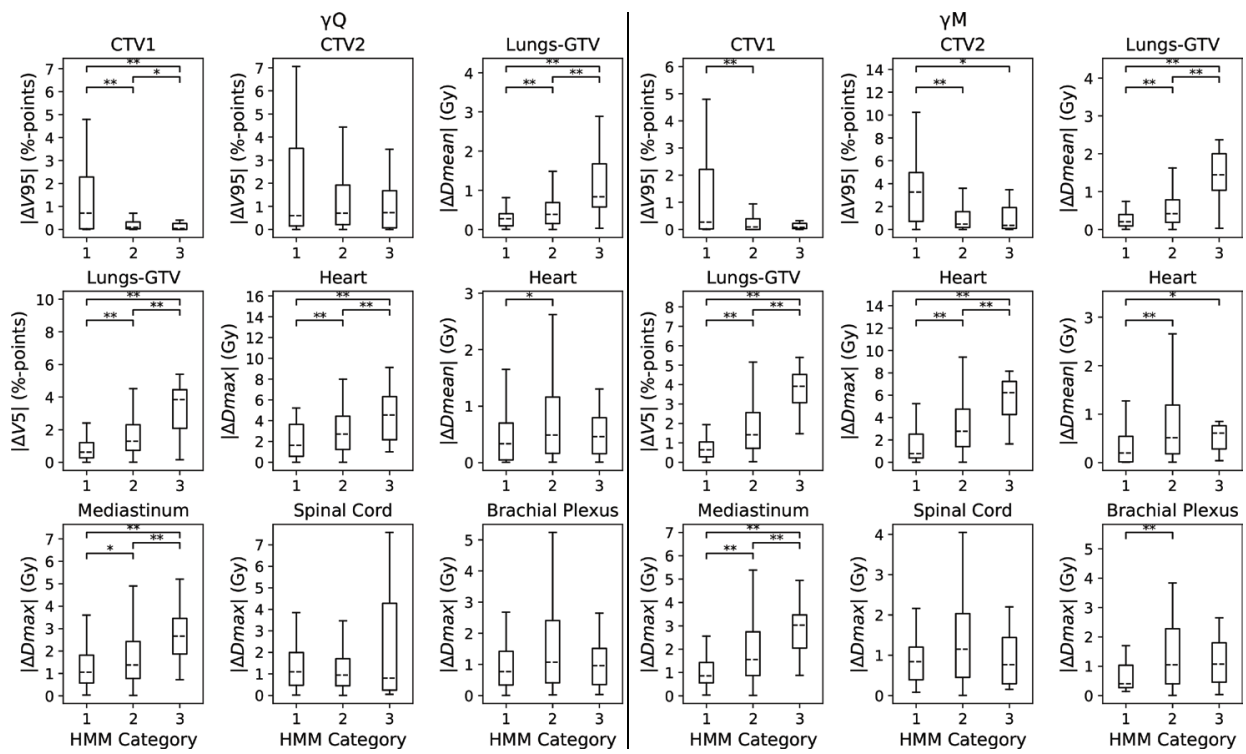

Figure 8.6: Boxplots showing the ranges of deviations in DVH metrics (excluding outliers) for the different categories of anatomical change, as classified by the HMM. $x$-axis: HMM classification, $y$-axis: change in DVH metric. ${ }^{*}: p<0.05 ;{ }^{* *}: p<0.01$. 


\section{Discussion}

An HMM was originally developed at $\mathrm{CHUQ}$ to classify anatomical changes in lung cancer patients, using three metrics extracted from gamma maps of EPID measurements $(\bar{\gamma}$, $\sigma_{\gamma}$ and $\left.\gamma_{\text {top } 1 \%}\right)$. This HMM would replace a threshold classification method based on $\bar{\gamma}$ alone, which was a surrogate for clinical classification at CHUQ. The aim of the present study was to externally validate the HMM with data from Maastro, i.e., to evaluate if the HMM trained at CHUQ could be used to classify anatomical changes for Maastro patients, based on EPID measurements alone.

First, the HMM was validated using the $\bar{\gamma}$ threshold classification used at CHUQ. This validation is only moderately successful, as there are many false positives and false negatives when comparing HMM and $\bar{\gamma}$ threshold classification. Especially the false negatives would be an issue, as this may lead to less clinical action taken than is actually needed. GFR values show an increasing trend when classified in a higher category by the HMM. For the GFR, other thresholds than the conventionally used $10 \%$ could be beneficial for classifying data in the different categories.

The false positives and false negatives are likely due to fairly large underlying differences between the $\mathrm{CHUQ}$ and Maastro datasets. There were differences in treatment techniques (3D-CRT/IMRT vs. VMAT/Hybrid), EPID measurement frequency (daily vs. first three fractions and every $3^{\text {rd }}$ fraction) and EPID dosimetry and evaluation software (Varian Portal Dosimetry and in-house implemented gamma calculation ${ }^{24}$ vs. in-house developed software and gamma calculation ${ }^{25}$ ). When the Maastro data was prepared in the same manner as at CHUQ $(\gamma Q)$, the performance of the HMM was slightly better than when the data was prepared according to Maastro's workflow $(\gamma M)$. It is likely that HMM performance compared to the $\bar{\gamma}$ threshold classification will increase further when other differences between the datasets are resolved. For instance, by re-training the HMM on a subset of the CHUQ dataset, only containing features of the fraction numbers used for EPID measurements at Maastro (i.e., $1^{\text {st }}, 2^{\text {nd }}$, $3^{\text {rd }}$ and every $3^{\text {rd }}$ fraction thereafter). This would align the EPID measurement frequency in the two datasets.

However, the $\bar{\gamma}$ threshold classification is not necessarily an indicator for anatomical changes in the Maastro data, as it is for the CHUQ data. Therefore, in a second stage, HMM classification was compared with Maastro's clinically used IGRT classification, to determine whether the HMM could be used to classify anatomical changes at Maastro, based on EPID measurements alone. This validation is not successful, as HMM and IGRT classification do not correspond. Hence, the HMM trained at CHUQ cannot be used to classify anatomical changes at Maastro.

If the aim is to use EPID measurements and HMMs to classify anatomical changes instead of the IGRT strategy currently in place, the HMM would have to be retrained with Maastro data. However, as Maastro's IGRT protocol is not triggered by EPID measurement results, it may be difficult to relate gamma metrics and IGRT classification, even when training a new HMM with Maastro data. A better approach might be to use classification based on DVH metrics or biological metrics such as TCP and NTCP values as reference. Yet, these approaches also have their difficulties as there is often no consensus on which thresholds to use. In this work, DVH metrics were only available for the Maastro dataset, and not for the CHUQ dataset, hence using them as a reference throughout was not possible. Still, as a first step, the $\Delta D V H$ values were 
considered to evaluate the relationship between gamma-based HMM classification and DVH metrics.

Nevertheless, it may be possible to use the HMM (without retraining it on Maastro data) in a different capacity at Maastro. 3D dose calculations on CBCT images (with propagated contours) were available for the Maastro data, and various $\triangle D V H$ values showed an increasing trend when classified in a higher category by the HMM. It is possible to relate HMM classification based on gamma metrics to increasing DVH differences for the larger OARs, but not for the target volumes and smaller OARs. Although there is overlap between the ranges of the $\triangle \mathrm{DVH}$ values for the three HMM categories for the larger OARs as well, the differences between the categories are still statistically significant. If a fraction is classified by the HMM in a higher category, this can serve as an indication that there are larger differences in the $\triangle \mathrm{DVH}$ values of the large OARs. This implies that the HMM could be used at Maastro as a supplementary source of information, next to IGRT classification of anatomical changes. The HMM classification would serve as a fast indicator for changes in the 3D dose. This could help with selecting patients needing a full 3D dose recalculation, and to avoid unnecessary recalculations.

It should be noted that there are uncertainties in this work that should be taken into account. On the one hand, the EPID measurements have an uncertainty, which is currently unknown. Although both CHUQ and Maastro use EPIDs from the same vendor (Varian Medical Systems), the EPID dosimetry software and implementation of gamma analysis are different, which may cause some differences between the datasets ${ }^{26,27}$. Little research has been performed to uncover the uncertainties when using the EPID as a dosimeter, although these are essential to incorporate in the development of robust, objective decision protocols. This is especially important in this work as the uncertainties in EPID measurements can propagate into the gamma analysis results ${ }^{28}$. This uncertainty is partially mitigated by using $\mathrm{YQ}$, in which EPID measurements are compared to another EPID measurement instead of an EPID dose prediction. Using less strict gamma criteria $((5 \%, 3 \mathrm{~mm})$ vs. $(3 \%, 3 \mathrm{~mm}))$ when comparing with an EPID dose prediction seems to have a similar effect, as the results of $\gamma \mathrm{Q}$ and $\gamma \mathrm{M}$ are similar. Another factor influencing the interpretation of the EPID measurements is that other errors than anatomical changes may be present. For instance, linac errors can occur simultaneously, although these are likely small due to regular linac QA measurements.

On the other hand, there are uncertainties in the CBCT dose recalculation process, associated both with the contour propagation and the dose calculation itself. The propagation of small structures and the CTV, as well as the use of CBCT HUs for the dose calculation can introduce uncertainties, although the latter only has a small contribution for Varian CBCT images ${ }^{29,30}$. Furthermore, some of the DVH metrics analyzed, such as the $D_{\text {max }}$ are sensitive to the inherent noise of the Monte Carlo dose calculation. As the $95 \%$ isodose line is optimized around the CTV during treatment planning, a small change in dose can have large consequences in the value of the $\mathrm{V}_{95 \%}$. This may be the reason that there is no trend towards larger differences in this metric with classification in higher categories by the HMM.

This work illustrates the challenges of applying a model to aid with decisions in adaptive radiotherapy that was developed in one center to data of another center. Underlying differences in data, but also in clinical protocols and clinical interpretation, 
makes transferring models difficult. In general, more multi-centric validation of EPID based methods is justified.

\section{Conclusions}

Concluding, the external validation shows that transferring the HMM for anatomical change classification to a different center is challenging, but can still be valuable. The HMM trained at CHUQ cannot be used directly to classify anatomical changes in the Maastro data. However, it may be possible to use the model in a different capacity, as an indicator for changes in the 3D dose based on 2D EPID measurements.

\section{Acknowledgements}

This study was supported in part by Varian Medical Systems (project: Decision DGRT-I) as well as by ESTRO (ESTRO Mobility Grant - Technology Transfer Grant). The authors would like to thank Dr. Stefan Scheib and Dr. Christof Baltes from Varian Medical Systems for commenting on the manuscript. 


\section{References}

1. van Zwienen M, van Beek S, Belderbos J, van Kranen S, Rasch C, van Herk M, and Sonke J. Anatomical changes during radiotherapy of lung cancer patients. International Journal of Radiation Oncology • Biology • Physics, 2008. 72(1): p. S111.

2. Kwint M, Conijn S, Schaake E, Knegjens J, Rossi M, Remeijer P, Sonke J-J, and Belderbos J. Intra thoracic anatomical changes in lung cancer patients during the course of radiotherapy. Radiotherapy and Oncology, 2014. 113(3): p. 392-397.

3. Møller DS, Khalil AA, Knap MM, and Hoffmann L. Adaptive radiotherapy of lung cancer patients with pleural effusion or atelectasis. Radiotherapy and Oncology, 2013. 110(3): p. 517-22.

4. Kataria T, Gupta D, Bisht S, Karthikeyan N, Goyal S, Pushpan L, Abhishek A, Govardhan H, Kumar V, and Sharma K. Adaptive radiotherapy in lung cancer: Dosimetric benefits and clinical outcome. The British Journal of Radiology, 2014. 87(1038): p. 20130643.

5. Persoon LCGG, Podesta M, Hoffmann L, Sanizadeh A, Schyns LEJR, de Ruiter BM, Nijsten SMJJG, Muren LP, Troost EGC, and Verhaegen F. Is integrated transit planar portal dosimetry able to detect geometric changes in lung cancer patients treated with volumetric modulated arc therapy? Acta Oncologica, 2015. 54(9): p. 1501-07.

6. Yan D, Vicini F, Wong J, and Martinez A. Adaptive radiation therapy. Physics in Medicine and Biology, 1997. 42(1): p. 123-32.

7. Nijsten SMJJG, van Elmpt WJ, Jacobs M, Mijnheer BJ, Dekker AL, Lambin P, and Minken AW. A global calibration model for a-Si EPIDs used for transit dosimetry. Medical Physics, 2007. 34(10): p. 3872-84.

8. van Elmpt W, McDermott L, Nijsten S, Wendling M, Lambin P, and Mijnheer B. A literature review of electronic portal imaging for radiotherapy dosimetry. Radiotherapy and Oncology, 2008. 88(3): p. 289-309.

9. Mans A, Wendling M, McDermott LN, Sonke J-J, Tielenburg R, Vijlbrief R, Mijnheer B, van Herk M, and Stroom JC. Catching errors with in vivo EPID dosimetry. Medical Physics, 2010. 37(6): p. 2638-44.

10. Persoon LCGG, Nijsten SMJJG, Wilbrink FJ, Podesta M, Snaith JAD, Lustberg T, van Elmpt WJC, van Gils F, and Verhaegen F. Interfractional trend analysis of dose differences based on 2D transit portal dosimetry. Physics in Medicine and Biology, 2012. 57(20): p. 6445-58.

11. Low DA, Harms WB, Mutic $S$, and Purdy JA. A technique for the quantitative evaluation of dose distributions. Medical Physics, 1998. 25(5): p. 656-61.

12. Low DA and Dempsey JF. Evaluation of the gamma dose distribution comparison method. Medical Physics, 2003. 30(9): p. 2455-64.

13. Olaciregui-Ruiz I, Rozendaal R, Mijnheer B, Van Herk M, and Mans A. Automatic in vivo portal dosimetry of all treatments. Physics in Medicine and Biology, 2013. 58(22): p. 8253.

14. Nelms BE, Zhen $\mathrm{H}$, and Tomé WA. Per-beam, planar IMRT QA passing rates do not predict clinically relevant patient dose errors. Medical Physics, 2011. 38(2): p. 1037-1044.

15. Waghorn BJ, Meeks SL, and Langen KM. Analyzing the impact of intrafraction motion: Correlation of different dose metrics with changes in target D95\%. Medical Physics, 2011. 38(8): p. 4505-11.

16. Thompson RF, Valdes G, Fuller CD, Carpenter CM, Morin O, Aneja S, Lindsay WD, Aerts HJ, Agrimson B, and Deville Jr C. Artificial intelligence in radiation oncology: A specialty-wide disruptive transformation? Radiotherapy and Oncology, 2018. 129(3): p. 421-426.

17. Varfalvy N, Piron O, Cyr MF, Dagnault A, and Archambault L. Classification of changes occurring in lung patient during radiotherapy using relative $\gamma$ analysis and hidden Markov models. Medical Physics, 2017. 44(10): p. 5043-5050.

18. Rabiner LR. A tutorial on hidden Markov models and selected applications in speech recognition. Proceedings of the IEEE, 1989. 77(2): p. 257-286.

19. Park SH and Han K. Methodologic guide for evaluating clinical performance and effect of artificial intelligence technology for medical diagnosis and prediction. Journal of 
Radiology, 2018. 286(3): p. 800-809.

20. van Elmpt WJC, Nijsten SMJJG, Mijnheer BJ, and Minken AWH. Experimental verification of a portal dose prediction model. Medical Physics, 2005. 32(9): p. 2805-18.

21. Shapiro SS and Wilk MB. An analysis of variance test for normality (complete samples). Biometrika, 1965. 52(3/4): p. 591-611.

22. Hattu D, Mannens J, Van Elmpt W, De Ruysscher D, and Ollers M. OC-0307: Workflow optimization of image-guided adaptive radiotherapy in lung cancer patients. Radiotherapy and Oncology 2018. 127: p. S161.

23. van Elmpt W, Nijsten SMJJG, Schiffeleers RFH, Dekker ALAJ, Mijnheer BJ, Lambin P, and Minken AWH. A Monte Carlo based three-dimensional dose reconstruction method derived from portal dose images. Medical Physics, 2006. 33(7): p. 2426-34.

24. Chen M, Lu W, Chen Q, Ruchala K, and Olivera G. Efficient gamma index calculation using fast Euclidean distance transform. Physics in Medicine and Biology, 2009. 54(7): p. 2037.

25. Podesta M, Persoon LCGG, and Verhaegen F. A novel time dependent gamma evaluation function for dynamic 2D and 3D dose distributions. Physics in Medicine and Biology, 2014. 59(20): p. 5973-85.

26. Hussein $M$, Clark $C$, and Nisbet $A$. Challenges in calculation of the gamma index in radiotherapy-towards good practice. Physica Medica, 2017. 36: p. 1-11.

27. Taborda A, Stroom J, Baltes C, Seabra A, Dikaiou K, and Greco C. PO-0810: Absolute dose pretreatment portal dosimetry using the Varian MAASTRO implementation. Radiotherapy and Oncology, 2017. 123: p. S433-S434.

28. Baeza JA, Wolfs CJA, Nijsten SMJJG, and Verhaegen F. Validation and uncertainty analysis of a pre-treatment 2D dose prediction model. Physics in Medicine and Biology, 2018. 63(3): p. 035033.

29. de Smet M, Schuring D, Nijsten S, and Verhaegen F. Accuracy of dose calculations on kV cone beam CT images of lung cancer patients. Medical Physics, 2016. 43(11): p. 5934-5941.

30. Loi G, Fusella M, Lanzi E, Cagni E, Garibaldi C, lacoviello G, Lucio F, Menghi E, Miceli R, Orlandini LC, Roggio A, Rosica F, Stasi M, Strigari L, Strolin S, and Fiandra C. Performance of commercially available deformable image registration platforms for contour propagation using patient-based computational phantoms: A multi-institutional study. Medical Physics, 2018. 45(2): p. 748-757. 


\section{Supplementary Material Chapter 8}

\section{Supplementary Material 8A: Results of the Shapiro-Wilk test for normality for the gamma fail rates in each HMM category}

The null hypothesis of this test is that the sample came from a normally distributed population. If the $p$-value is less than the significance level ( 0.05 in this case), the null hypothesis is rejected, serving as an indication that the data is not normally distributed. $p$-values $<0.05$ are marked in bold in Table 8A.1. The null hypothesis can be rejected for all cases except absolute gamma analysis and HMM category 3.

Table 8A.1: Results of the Shapiro-Wilk test for the gamma fail rates in each HMM category

\begin{tabular}{ccccc}
\hline \hline & \multicolumn{2}{c}{ Relative gamma analysis } & \multicolumn{2}{c}{ Absolute gamma analysis } \\
\hline \hline HMM Category & Test statistic W & $p$-value & Test statistic W & $p$-value \\
\hline 1 & 0.73 & $\mathbf{3 . 6 1} \cdot \mathbf{1 0}^{-\mathbf{1 0}}$ & 0.90 & $\mathbf{1 . 8 7 \cdot \mathbf { 1 0 }}$ \\
2 & 0.89 & $\mathbf{1 . 7 7} \cdot \mathbf{1 0}-\mathbf{9}$ & 0.89 & $\mathbf{6 . 9 8 \cdot 1 0 ^ { - 1 1 }}$ \\
3 & 0.90 & $\mathbf{2 . 4 0 \cdot 1 0 ^ { - 3 }}$ & 0.93 & 0.32 \\
\hline
\end{tabular}


Supplementary Material 8B: Results of the Shapiro-Wilk test for normality for the $\triangle D V H$ values in each HMM category

The null hypothesis of this test is that the sample came from a normally distributed population. If the $p$-value is less than the significance level (0.05 in this case), the null hypothesis is rejected, serving as an indication that the data is not normally distributed. $p$-values $<0.05$ are marked in bold in Table 8B.1. The null hypothesis can be rejected in most cases.

Table 8B.1: Results of the Shapiro-Wilk test for the $\triangle \mathrm{DVH}$ values in each HMM category

\begin{tabular}{|c|c|c|c|c|c|}
\hline \multirow[b]{2}{*}{ DVH metric } & \multirow[b]{2}{*}{$\begin{array}{c}\text { HMM } \\
\text { Category }\end{array}$} & \multicolumn{2}{|c|}{ Relative gamma analysis } & \multicolumn{2}{|c|}{ Absolute gamma analysis } \\
\hline & & $\begin{array}{c}\text { Test statistic } \\
\text { W }\end{array}$ & $p$-value & $\begin{array}{c}\text { Test statistic } \\
\text { W }\end{array}$ & $p$-value \\
\hline \multirow{3}{*}{$\begin{array}{l}\text { CTV1 } \\
\mathrm{V}_{95 \%}\end{array}$} & 1 & 0.57 & $5.74 \cdot 10^{-12}$ & 0.54 & $4.94 \cdot 10^{-12}$ \\
\hline & 2 & 0.43 & $1.05 \cdot 10^{-20}$ & 0.31 & $1.17 \cdot 10^{-24}$ \\
\hline & 3 & 0.24 & $5.34 \cdot 10^{-13}$ & 0.49 & $9.19 \cdot 10^{-6}$ \\
\hline \multirow{3}{*}{$\begin{array}{l}\text { CTV2 } \\
\mathrm{V}_{95 \%}\end{array}$} & 1 & 0.68 & $1.72 \cdot 10^{-9}$ & 0.81 & $1.69 \cdot 10^{-5}$ \\
\hline & 2 & 0.51 & $8.29 \cdot 10^{-17}$ & 0.44 & $5.97 \cdot 10^{-16}$ \\
\hline & 3 & 0.47 & $1.67 \cdot 10^{-9}$ & 0.70 & $3.66 \cdot 10^{-3}$ \\
\hline \multirow{3}{*}{$\begin{array}{c}\text { Lungs - GTV } \\
D_{\text {mean }}\end{array}$} & 1 & 0.81 & $3.82 \cdot 10^{-8}$ & 0.90 & $3.06 \cdot 10^{-4}$ \\
\hline & 2 & 0.90 & $6.00 \cdot 10^{-9}$ & 0.76 & $1.65 \cdot 10^{-16}$ \\
\hline & 3 & 0.90 & $1.69 \cdot 10^{-3}$ & 0.96 & 0.70 \\
\hline \multirow{3}{*}{$\begin{array}{c}\text { Lungs - GTV } \\
\mathrm{V}_{5 \mathrm{~Gy}}\end{array}$} & 1 & 0.87 & $2.67 \cdot 10^{-6}$ & 0.91 & $7.58 \cdot 10^{-4}$ \\
\hline & 2 & 0.91 & $8.65 \cdot 10^{-8}$ & 0.91 & $3.93 \cdot 10^{-9}$ \\
\hline & 3 & 0.89 & $1.91 \cdot 10^{-3}$ & 0.92 & 0.23 \\
\hline \multirow{3}{*}{$\begin{array}{l}\text { Heart } \\
D_{\text {mean }}\end{array}$} & 1 & 0.55 & $1.78 \cdot 10^{-13}$ & 0.48 & $9.31 \cdot 10^{-13}$ \\
\hline & 2 & 0.56 & $1.68 \cdot 10^{-19}$ & 0.58 & $1.81 \cdot 10^{-21}$ \\
\hline & 3 & 0.92 & $9.25 \cdot 10^{-3}$ & 0.91 & 0.19 \\
\hline \multirow{3}{*}{$\begin{array}{l}\text { Heart } \\
\mathrm{D}_{\max }\end{array}$} & 1 & 0.82 & $6.40 \cdot 10^{-8}$ & 0.76 & $2.66 \cdot 10^{-8}$ \\
\hline & 2 & 0.88 & $1.01 \cdot 10^{-9}$ & 0.85 & $1.06 \cdot 10^{-12}$ \\
\hline & 3 & 0.66 & $3.33 \cdot 10^{-8}$ & 0.89 & 0.10 \\
\hline \multirow{3}{*}{$\begin{array}{l}\text { Mediastinum } \\
\qquad D_{\max }\end{array}$} & 1 & 0.90 & $4.62 \cdot 10^{-5}$ & 0.96 & 0.04 \\
\hline & 2 & 0.90 & $1.84 \cdot 10^{-8}$ & 0.93 & $2.72 \cdot 10^{-8}$ \\
\hline & 3 & 0.95 & 0.10 & 0.98 & 0.95 \\
\hline \multirow{3}{*}{$\begin{array}{l}\text { Spinal cord } \\
\mathrm{D}_{\max }\end{array}$} & 1 & 0.81 & $7.35 \cdot 10^{-8}$ & 0.71 & $5.18 \cdot 10^{-9}$ \\
\hline & 2 & 0.78 & $1.35 \cdot 10^{-13}$ & 0.73 & $4.43 \cdot 10^{-17}$ \\
\hline & 3 & 0.73 & $1.16 \cdot 10^{-6}$ & 0.88 & 0.08 \\
\hline \multirow{3}{*}{$\begin{array}{c}\text { Brachial plexus } \\
D_{\max }\end{array}$} & 1 & 0.36 & $1.06 \cdot 10^{-10}$ & 0.85 & $6.20 \cdot 10^{-3}$ \\
\hline & 2 & 0.58 & $1.12 \cdot 10^{-12}$ & 0.57 & $6.56 \cdot 10^{-15}$ \\
\hline & 3 & 0.50 & $1.39 \cdot 10^{-6}$ & 0.55 & $4.08 \cdot 10^{-5}$ \\
\hline
\end{tabular}




\section{Supplementary Material $8 C: \Delta D V H$ results including outliers}
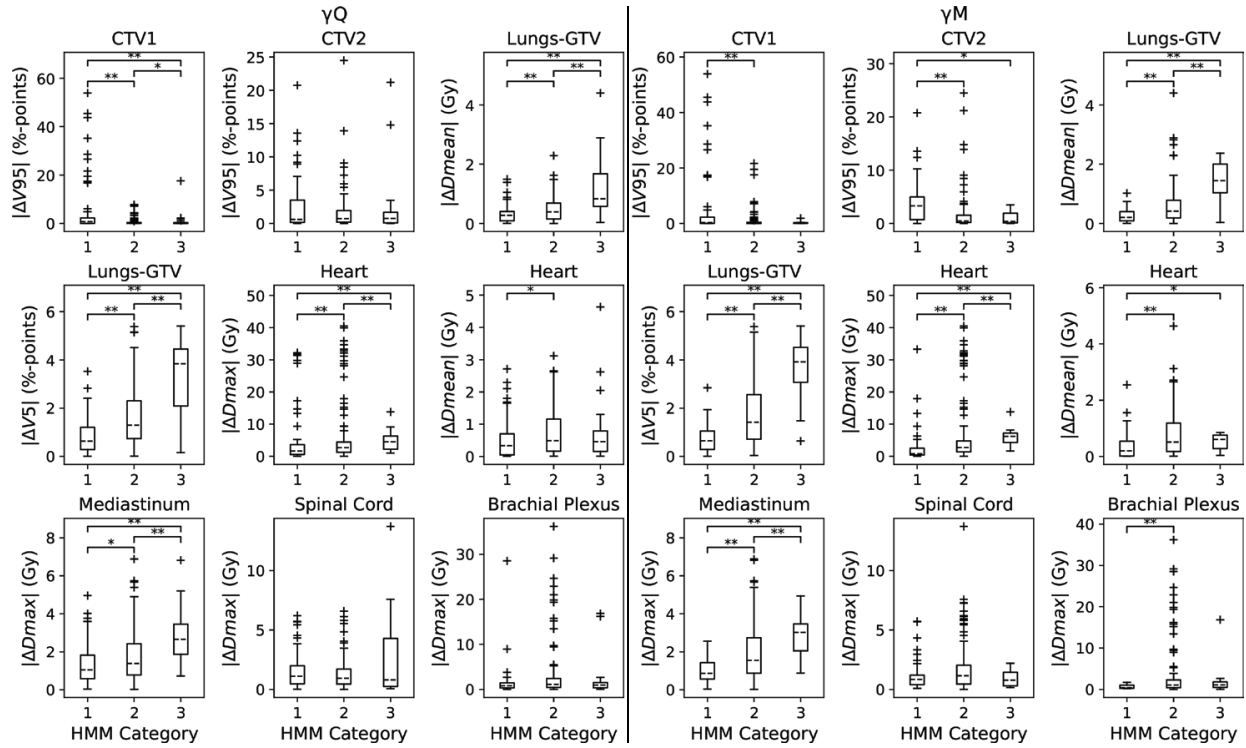

Figure 8C.1: Boxplots showing the ranges of deviations in DVH metrics (including outliers) for the different categories of anatomical change, as classified by the HMM. $x$-axis: HMM classification, $y$-axis: change in DVH metric, ${ }^{*}: p<0.05,{ }^{* *}: p<0.01$. 



\section{Chapter 9}

Discussion and future perspectives 

Portal dosimetry using electronic portal imaging devices (EPIDs) for verification of complex radiotherapy treatments has been subject of research for many years already'. Nowadays, nearly every linac is equipped with an EPID². Technically, portal dosimetry is very advanced, with the possibility of verifying treatments in $2 D, 3 D$ and even 2D+time and 4D. However, practically, it is still not widely used for in vivo treatment verification. Its clinical acceptance has been hampered by the unknown uncertainties of the methods, as well as by the lack of effective, automated clinical decision protocols that are sensitive to relevant dosimetric errors. The aim of this thesis was therefore to investigate the error detection capabilities of portal dosimetry methods, including uncertainties and advanced methods for error classification.

\section{Part I: Uncertainty and sensitivity of portal dosimetry methods}

Little research has been performed into the uncertainties of portal dosimetry methods. Yet, when aiming to use portal dosimetry results as input for clinical decisions, it is vital to know their random and systematic errors. Especially when using threshold based methods, as is common in clinical practice, an action threshold needs to be flexible enough to incorporate the uncertainty. Otherwise, for every flagged dose deviation, it is unclear if an actual dose error has occurred, or if it is only due to the uncertainty of the method.

Table 9.1 gives a non-exhaustive overview of the steps in the portal dosimetry workflow, the involved methods and modalities, and their associated uncertainties. Different kinds of uncertainties can be distinguished. For instance, when using a cone beam CT (CBCT) image for a 3D dose calculation, geometrical uncertainties can arise as the CBCT is often stitched to a CT image to increase the field of view. Hounsfield unit $(\mathrm{HU})$ uncertainties and their propagation into the dose distribution are also more pronounced in CBCT images and have been quantified by de Smet et al. ${ }^{3}$ When using a Monte Carlo dose engine, it is well known that there is a statistical uncertainty that depends on the number of particles used for simulation, which is a tradeoff with the computation time ${ }^{4}$. Furthermore, the EPID itself has a random uncertainty related to reproducibility, i.e., there will be small fluctuations in the EPID signal such that irradiating the same field multiple times will result in slightly different results.

The work in this thesis has additionally provided a framework for analyzing portal dosimetry model uncertainties (Chapter 2), which is another step towards quantifying the overall uncertainty of portal dosimetry results for objective decision protocols. This framework was applied to a larger dataset in Chapter 3, showing that the model uncertainty is influenced by the presence of small radiation fields, which are becoming more common as treatment complexity increases. The uncertainty was only assessed for the simplest model in the portal dosimetry chain: the 2D pre-treatment dose prediction model ${ }^{5}$. This analysis should be repeated to evaluate the uncertainty of other models in the portal dosimetry workflow as well ${ }^{6-9}$. Likely, the uncertainty will become larger as the models become more complex.

All these uncertainties propagate through the portal dosimetry workflow and ultimately affect the dose comparison results, both for gamma analysis and dosevolume histogram (DVH) metrics ${ }^{10,11}$. Gamma analysis results are also influenced by the software implementation used ${ }^{12}$, and uncertainties related to contouring, e.g., when using contour propagation methods ${ }^{13}$, can have an effect on the values of DVH 
metrics. This stresses the importance of rigorous evaluation and quantification of all uncertainties to improve objective interpretation of portal dosimetry results and the development of effective decision protocols for adaptive radiotherapy.

Besides the uncertainty of the portal dosimetry methods, it is also important to investigate their sensitivity and specificity, i.e., the error magnitude that can be detected as well as the methods' ability to detect relevant dose errors without generating too many false negatives and false positives. Common clinically used gamma criteria and action thresholds (i.e., $(3 \%, 3 \mathrm{~mm})$ gamma analysis with a $10 \%$ gamma fail rate threshold), may not be optimal for this task. The sensitivity and specificity of several portal dosimetry methods for detecting simulated treatment errors using a simple threshold based classification method was investigated in Chapter 4. It was shown that different combinations of portal dosimetry methods, gamma criteria and gamma fail rate thresholds are optimal for detecting different types of errors.

The interest in sensitivity and specificity of portal dosimetry methods is reflected by the increasing number of publications on this topic ${ }^{14-20}$. These studies show

Table 9.1: Steps in the portal dosimetry workflow and associated uncertainties. CT: computed tomography, CBCT: cone beam CT, HU: Hounsfield unit, EPID: electronic portal imaging device, DVH: dose-volume histogram

\begin{tabular}{lll}
\hline \hline $\begin{array}{l}\text { Step in portal } \\
\text { dosimetry } \\
\text { workflow }\end{array}$ & Methods and modalities & Uncertainties \\
\hline \hline \multirow{5}{*}{ Dose prediction } & $\begin{array}{l}\text { Dose prediction models } \\
\text { (pre-treatment \& transit) }\end{array}$ & Model uncertainty \\
\cline { 2 - 3 } & CT/CBCT image & $\begin{array}{l}\text { Geometrical uncertainties } \\
\text { HU uncertainty }\end{array}$ \\
\cline { 2 - 3 } & $\begin{array}{l}\text { Dose calculation engine } \\
\text { (In this work: Monte Carlo) }\end{array}$ & $\begin{array}{l}\text { Statistical uncertainty } \\
\text { Beam model uncertainty }\end{array}$ \\
\hline & EPID & Reproducibility \\
\cline { 2 - 3 } Dose acquisition & $\begin{array}{l}\text { Conversion model: EPID } \\
\text { images to 2D (+ time) } \\
\text { dose distributions }\end{array}$ & $\begin{array}{l}\text { EPID calibration } \\
\text { Model uncertainty }\end{array}$ \\
\cline { 2 - 3 } & CT/CBCT image & $\begin{array}{l}\text { Geometrical uncertainties } \\
\text { HU uncertainty }\end{array}$ \\
\cline { 2 - 3 } & $\begin{array}{l}\text { Dose calculation engine } \\
\text { (In this work: Monte Carlo) }\end{array}$ & $\begin{array}{l}\text { Statistical uncertainty } \\
\text { Beam model uncertainty }\end{array}$ \\
\hline & & $\begin{array}{l}\text { Implementation of gamma } \\
\text { calculation } \\
\text { Propagation of uncertainties in } \\
\text { previous steps }\end{array}$ \\
\hline \multirow{2}{*}{ Dose comparison } & $\begin{array}{l}\text { Contour propagation uncertainty } \\
\text { Propagation of uncertainties in } \\
\text { previous steps }\end{array}$ \\
\cline { 2 - 3 } & DVH metrics &
\end{tabular}


on the one hand what the error detection capabilities of portal dosimetry methods are, and on the other hand how to optimize threshold based classification models for improved error detection. Both of these aspects are relevant for the development of clinical decision protocols based on portal dosimetry, but until recently, this knowledge was lacking. This hindered the implementation of effective decision protocols and the widespread use of portal dosimetry for treatment verification. Now that an increasing number of these studies is performed, the capabilities and limitations of portal dosimetry methods become more defined, which also provides guidance for users on how to interpret portal dosimetry results and how to develop effective decision protocols.

\section{Part II: Advanced methods for error classification based on portal dosimetry}

The application of artificial intelligence (Al) to portal dosimetry for error classification is a recent development, with only a few publications available ${ }^{17,21,22}$. However, $\mathrm{Al}$ algorithms may be especially suited to overcome the problems associated with simple threshold classification methods for portal dosimetry. This may cause a big leap forward in the development of effective protocols for clinical decision making in adaptive radiotherapy, help automate the in vivo dosimetry process and therefore improve the clinical use of portal dosimetry.

Figure 9.1 provides a schematic overview of the elements involved in a dose-guided adaptive radiotherapy workflow, and how an Al-based error detection workflow can be added. An adaptive workflow as shown here is often only started after visual assessment of a CBCT image, hence not for every fraction, especially when this workflow is not (fully) automated. Although a great amount of daily information can be included for dose recalculation, e.g., a CBCT image for the anatomy of the day or log files for the actual values of linac parameters, this approach cannot detect

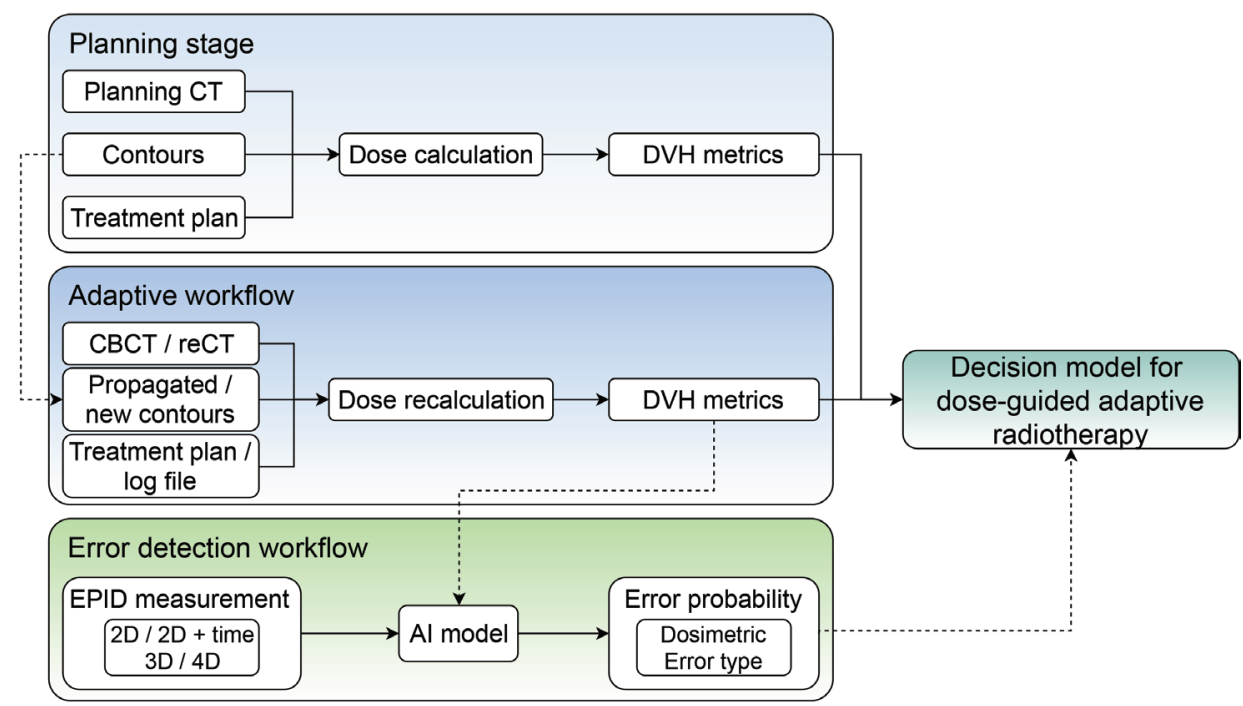

Figure 9.1: Overview of an adaptive workflow and an Al-based error detection workflow. CT: computed tomography, CBCT: cone beam CT; reCT: repeated CT; Al: artificial intelligence. 
anatomical changes or patient motion occurring during treatment delivery. An in vivo dose measurement is needed to record that information, for which the EPID is the most flexible solution.

A benefit of the EPID is that it provides 'free' information. As the exit radiation is recorded during treatment, no additional dose has to be given to the patient and no time has to be added to the treatment fraction. After installation of portal dosimetry software and calibration of the EPID, it is therefore easy to use daily. The information obtained in an Al-based error detection workflow using portal dosimetry can be beneficial to quickly detect various treatment errors and to analyze the probabilities that certain errors occurred. This would be a substantial new source of information that can be used in decision models for dose-guided adaptive radiotherapy.

This new information source can be combined with information from daily dose recalculations using $\mathrm{CBCT}$ images and log files. However, if $\mathrm{CBCT}$ imaging and dose recalculation cannot be performed daily, e.g., due to patient load or time constraints, an Al and portal dosimetry based error detection workflow can particularly be of added value. Even on days when there is no CBCT available, an error detection workflow as proposed here can give an indication of the treatment deviations that occurred, without increasing treatment time or workload.

In the second part of this thesis, several Al methods and classification problems were investigated to show the potential of Al-based error detection for portal dosimetry, and to contribute to the development of the proposed error detection workflow. Although the results look promising for the development of advanced decision protocols, there are some concerns that should be addressed.

A well-known phrase in the field of Al is "garbage in = garbage out", which means that the predictions that an Al model makes, are only as good as the data that is used as input. On the one hand, this is where the two parts of this thesis connect. A high uncertainty in the portal dosimetry results may influence the performance of any model that uses this data as input. However, the exact value of the uncertainty for the full portal dosimetry workflow is not known, and its effect on the performance of $\mathrm{Al}$ models for error classification needs to be examined in detail.

On the other hand, the "garbage in = garbage out" principle also applies to the ground truth classification. For the classification problem presented in Chapters 5 and 6, i.e., classifying gamma analysis results into relevant and irrelevant dose deviations, determining what the ground truth classification should be (i.e., what actually constitutes a relevant dose deviation) is problematic. DVHs are most often used to evaluate dose distributions and dose deviations clinically, but it is difficult to reach consensus about which DVH metrics and action thresholds to use.

In this thesis two different ground truth classification options are used. In Chapter 5, the difference in $\mathrm{D}_{95 \%}$ of the GTV was evaluated, whereas in Chapter 6, the planning objectives and constraints were considered as action thresholds. In the former case, the doses to organs-at-risk (OARs) are not taken into account, and tumor coverage is usually assessed by the dose to the CTV or PTV instead of the dose to the GTV. In the latter case, large dose deviations could go undetected as long as their values remain within the planning objectives and constraints. An example of this would be an OAR with a very low dose in the planned dose distribution. It is possible that this dose increases vastly over the course of treatment, but not enough to reach the planning 
constraint. Such a deviation is undesirable clinically and it would go undetected by the DVH action threshold, but may show up in gamma analysis. Hence, a clear ground truth classification based on DVH metrics is not available, and this impedes development of objective decision models.

Tumor control probability (TCP) and normal tissue complication probability (NTCP) models may provide a more objective reference for this classification problem. Although these models have their own associated uncertainties and limitations, an extensive evaluation and validation, particularly of NTCP models, has been performed for various tumor sites for the model-based approach to proton therapy in the Netherlands $s^{23,24}$. In this context, consensus has been reached on which OARs to take into account and action thresholds for NTCP values have been set. These could be used to determine objective thresholds for relevant dose deviations in photon therapy as well.

As an alternative, the classification problem can be altered, as was shown in Chapter 7. Instead of classifying gamma maps into relevant and irrelevant dose deviations, gamma maps are classified into error types and even error magnitudes. This approach provides additional information that is not currently available in the clinical portal dosimetry workflow. Ideally, both classification problems should be combined, such that both the error type and magnitude as well as the dosimetric implications of the error can be provided for clinical decision making.

An important aspect of $\mathrm{Al}$ is model validation. An Al model can perform very well on the data it is developed on, but it can break down on new data. This problem is especially prominent when applying a model that is developed at a different institute, i.e., with different acquisition parameters for the input data. In Chapter 8, an external validation of a hidden Markov model (HMM) for anatomical change classification was therefore performed. This model was developed at CHU de Québec (Canada) as the first application of machine learning to portal dosimetry, and validated with data from Maastro. This study showed that it is difficult to use the HMM on clinical data acquired in a different institute for the same classification purpose.

An HMM is an example of an unsupervised machine learning method. This can be beneficial in the portal dosimetry context, as there is no ground truth classification needed. The model groups the data and clinical meaning can be attached to these groups afterwards. In Chapter 8, a relationship was observed between HMM classification based on features extracted from gamma maps and differences in DVH metrics for large OARs such as the lungs, heart and mediastinum. A disadvantage is that the HMM as used in this thesis cannot take the full gamma map into account. A hybrid deep neural network - HMM or other unsupervised deep learning techniques would be capable of taking images or volumes as input and might be a potential solution to this problem ${ }^{25,26}$.

\section{Future perspectives}

As the studies in this thesis are among the first to evaluate the uncertainties of portal dosimetry methods and to apply Al methods for error classification based on portal dosimetry, there are many directions for future research still to be explored.

Previous work has shown that time-resolved portal dosimetry is more sensitive to treatment errors than time-integrated portal dosimetry, even when using 
threshold based classification methods ${ }^{27,28}$. One of the main difficulties with timeresolved dosimetry has been the interpretability of the results. As there is a multitude of doses per VMAT arc, the amount of information to process increases dramatically compared to time-integrated dosimetry. Determining action thresholds for timeresolved dosimetry therefore becomes an even more complex problem. Al algorithms can take large amounts of information into account, making them suitable candidates for error classification for time-resolved dosimetry as well.

For detailed error detection, the combination of time-resolved portal dosimetry and Al offers many possibilities. A particularly interesting approach would be to use 2D time-resolved doses as input for a deep learning model, and include projections of relevant structures onto the 2D planes. This would add even more spatial information, which can be beneficial for error type, error magnitude and error relevance classification with Al. It would allow to pinpoint which error has occurred, where it has occurred and when it has occurred, in an automated way and without having to perform a full 3D dose calculation.

Although gamma analysis is a common method for dose comparison in radiotherapy, its results are dependent on many factors, such as software implementation, gamma criteria, local or global analysis and resolution of the images/ volumes to be compared ${ }^{12}$. All Al methods in this thesis used gamma maps, or features extracted from gamma maps, as input. However, for deep learning methods, it may also be possible to input both the planned and delivered dose instead. That way, the dose comparison would be incorporated into the deep learning model, overcoming issues associated with gamma analysis.

The work in this thesis has mostly focused on lung cancer patients. As the density of the lung compared to that of tumor and surrounding tissues are very different from each other, anatomical changes quickly lead to differences in the EPID measurements. For other tumor sites, the EPID may be less sensitive to anatomical changes. In Chapter 3, it was shown that there are differences in the uncertainties of portal dosimetry methods for different tumor sites. For error detection and classification, it is likely that different models per tumor site are needed, as there are different errors that can occur as well as different DVH metrics that are relevant for each tumor site.

In this thesis, Al methods were applied to the $2 \mathrm{D}$ in vivo part of the portal dosimetry workflow, and the focus was on error classification. However, there are also other parts of the portal dosimetry workflow that can benefit from Al. Several studies have examined the prediction of gamma pass or fail rates for patient specific quality assurance $(\mathrm{QA})$ based on treatment plan characteristics or 2D dose distributions, also when using the EPID for this purpose ${ }^{29-32}$. In clinical practice, every treatment plan undergoes pre-treatment QA, causing a high workload as each plan has to be irradiated without the patient present. Prediction of gamma pass/fail rates can reduce this workload, as plans that are predicted to pass gamma analysis by the AI model may not need pre-treatment irradiation.

Another application would be the conversion from EPID images to portal dose images with a deep learning algorithm. There are a few examples of this in literature for the pre-treatment scenario ${ }^{33,34}$. These studies use artificial neural networks to recreate the treatment planning system (TPS) dose from EPID measurements. A disadvantage 
of that approach is that the EPID dose is not independent from the TPS. Training with an independent 2D dose measurement as ground truth would be better. However, it is difficult to obtain a $2 \mathrm{D}$ dose measurement with a high spatial resolution, yet a low uncertainty. Commonly used 2D detectors have a lower spatial resolution than the EPID, while film has a high spatial resolution but generally a higher uncertainty and it can only measure dose in a time-integrated manner ${ }^{35}$. Additionally, it remains to be investigated if Al-based EPID conversion models are more accurate than the models that are currently in use, which would require another independent measurement to validate both types of models.

For 3D and 4D dose calculations, a Monte Carlo dose engine can be used in a portal dosimetry workflow. Although Monte Carlo doses can be very accurate, it is well-known that doses with low noise levels require long computation times, that are not feasible in clinical practice. Thus, a trade-off needs to be made between the level of noise in the dose distribution and the computation time. Variance reduction techniques and increasing availability of powerful hardware can help in this respect ${ }^{4}$. However, another approach would be to use deep learning to remove noise from the dose distributions. The input for the model would be a dose distribution with a high noise level, and the ground truth a dose distribution with a low noise level. Javaid et al. ${ }^{36}$ showed the feasibility of this approach using a U-Net with 2D and 2.5D input (i.e., a single slice of the 3D dose distribution or multiple adjacent slices). Their method could provide an accurate dose with low noise in less than 20 seconds (combined calculation and U-Net inference time), compared to up to 100 minutes for a Monte Carlo calculation with more particles. As DVH metrics used for clinical decision making can be influenced by noise ${ }^{10}$, implementing a fast Al-based method for noise removal could improve reliability of DVH results and in turn improve error detection and decision making.

Radiotherapy is a fast evolving field, where new technologies are adopted rapidly. However, for the clinical implementation of Al models there are still hurdles to overcome ${ }^{37-41}$. Al algorithms are often treated as black boxes, making it difficult to interpret intermediate steps of deep learning algorithms, which influences clinical acceptance. If they are implemented, quality assurance is needed for these methods, just like other equipment used in clinical practice. A limiting factor in model development is the amount of high quality data that is needed to obtain a wellperforming, generalizable model. This is often difficult to obtain in clinical settings, and in the case of error detection data may be highly imbalanced, i.e., few cases may exhibit errors. Data sharing between institutes can be difficult due to patient privacy issues on the one hand, and differences in data acquisition methodology on the other hand. Guidelines for the development and implementation of Al models in clinical practice in radiation oncology, addressing these issues, should be established by international organizations such as the European Society for Radiotherapy and Oncology (ESTRO).

With regards to improving the widespread use of portal dosimetry for error detection, there is also a role for vendors of portal dosimetry software. There are several software packages available ${ }^{42}$, but these are often not the plug-and-play solutions they promise to be. Specifications about uncertainties and which error types and magnitudes can be detected and which cannot, are usually not provided. Each institute needs to determine their own action thresholds, which are often conservative 
to avoid missing relevant errors (false negatives), but in turn can lead to unreasonably high workloads due to false positives. This results in underutilization of a potentially very effective treatment verification method. Therefore, vendors should also make an effort to report the uncertainties, sensitivity and specificity of their software, such that it will become easier for the users to develop clinical decision models.

By including pre-trained Al models in portal dosimetry software packages, the clinical use of portal dosimetry for error detection can be improved as well. Vendors should again clearly communicate the capabilities and limitations of such models. Ideally, users of the software would have the choice to use a provided Al model as is (i.e., as a real plug-and-play solution), or to fine-tune it further to their own institutional guidelines. An error type classification model is particularly suitable to be included in a portal dosimetry software package, as it can be pre-trained to recognize error types, which are not dependent on the institute where the software and model are used. Relevant error magnitudes can be institute specific and the user should be able to optimize the model to detect the errors and error magnitudes that are relevant for their application. Developing an Al model for error relevance classification (e.g., when relating gamma analysis and DVH metrics) that can easily be used in multiple institutes will be more complicated, as what is considered the ground truth classification may vary between institutes. However, if it is clear how and why a certain ground truth classification is chosen, it is still possible to implement such a model in a portal dosimetry software package.

\section{Conclusion}

Overall, portal dosimetry for dose-guided radiotherapy can greatly benefit from improved error detection methods, which can be obtained by:

- Detailed examination of the uncertainties involved in the portal dosimetry workflow

- Rigorous investigation of the sensitivity and specificity of the portal dosimetry methods

- Advanced methods for error classification, such as Al algorithms

This thesis has contributed to these requirements, by providing a framework for uncertainty analysis of portal dosimetry methods (Chapters 2 and 3), by examining the sensitivity and specificity for threshold based classification methods for various portal dosimetry methods (Chapter 4), and by investigating various Al algorithms and error classification problems (Chapters 5, 6, 7 and 8).

Further development of these procedures and techniques, and integrating them in portal dosimetry workflows will help to improve error detection based on portal dosimetry, as well as the clinical use of portal dosimetry. In turn, this can benefit decision making for dose-guided adaptive radiotherapy, which will ultimately improve patient treatment. 


\section{References}

1. van Elmpt W, McDermott L, Nijsten S, Wendling M, Lambin P, and Mijnheer B. A literature review of electronic portal imaging for radiotherapy dosimetry. Radiotherapy and Oncology, 2008. 88(3): p. 289-309.

2. McCurdy B, Greer P, and Bedford J. Electronic portal imaging device dosimetry, in Clinical 3D Dosimetry in Modern Radiation Therapy, B. Mijnheer, Editor. 2017, CRC Press: Boca Raton, FL.

3. de Smet M, Schuring D, Nijsten S, and Verhaegen F. Accuracy of dose calculations on kV cone beam CT images of lung cancer patients. Medical Physics, 2016. 43(11): p. 5934-5941.

4. Chetty IJ, Curran B, Cygler JE, DeMarco JJ, Ezzell G, Faddegon BA, Kawrakow I, Keall PJ, Liu H, Ma C-MC, Rogers DWO, Seuntjens J, Sheikh-Bagheri D, and Siebers JV. Report of the AAPM Task Group No. 105: Issues associated with clinical implementation of Monte Carlo-based photon and electron external beam treatment planning. Medical Physics, 2007. 34(12): p. 4818-4853.

5. Baeza JA, Wolfs CJA, Nijsten SMJJG, and Verhaegen F. Validation and uncertainty analysis of a pre-treatment 2D dose prediction model. Physics in Medicine and Biology, 2018. 63(3): p. 035033.

6. van Elmpt WJC, Nijsten SMJJG, Mijnheer BJ, and Minken AWH. Experimental verification of a portal dose prediction model. Medical Physics, 2005. 32(9): p. 2805-18.

7. van Elmpt W, Nijsten SMJJG, Schiffeleers RFH, Dekker ALAJ, Mijnheer BJ, Lambin P, and Minken AWH. A Monte Carlo based three-dimensional dose reconstruction method derived from portal dose images. Medical Physics, 2006. 33(7): p. 2426-34.

8. Nijsten SMJJG, van Elmpt WJ, Jacobs M, Mijnheer BJ, Dekker AL, Lambin P, and Minken AW. A global calibration model for a-Si EPIDs used for transit dosimetry. Medical Physics, 2007. 34(10): p. 3872-84.

9. van Elmpt W, Nijsten S, Petit S, Mijnheer B, Lambin P, and Dekker A. 3D in vivo dosimetry using megavoltage cone-beam CT and EPID dosimetry. International Journal of Radiation Oncology • Biology • Physics, 2009. 73(5): p. 1580-1587.

10. Keall P, Siebers J, Jeraj R, and Mohan R. The effect of dose calculation uncertainty on the evaluation of radiotherapy plans. Medical Physics, 2000. 27(3): p. 478-484.

11. Graves YJ, Jia X, and Jiang SB. Effect of statistical fluctuation in Monte Carlo based photon beam dose calculation on gamma index evaluation. Physics in Medicine and Biology, 2013. 58(6): p. 1839.

12. Hussein $M$, Clark $C$, and Nisbet $A$. Challenges in calculation of the gamma index in radiotherapy-towards good practice. Physica Medica, 2017. 36: p. 1-11.

13. Loi G, Fusella M, Lanzi E, Cagni E, Garibaldi C, lacoviello G, Lucio F, Menghi E, Miceli R, Orlandini LC, Roggio A, Rosica F, Stasi M, Strigari L, Strolin S, and Fiandra C. Performance of commercially available deformable image registration platforms for contour propagation using patient-based computational phantoms: A multi-institutional study. Medical Physics, 2018. 45(2): p. 748-757.

14. Carlone M, Cruje C, Rangel A, McCabe R, Nielsen M, and MacPherson M. ROC analysis in patient specific quality assurance. Medical Physics, 2013. 40(4): p. 042103.

15. Bojechko C and Ford EC. Quantifying the performance of in vivo portal dosimetry in detecting four types of treatment parameter variations. Medical Physics, 2015. 42(12): p. 6912-6918.

16. Mijnheer B, Jomehzadeh A, González P, Olaciregui-Ruiz I, Rozendaal R, Shokrani P, Spreeuw $\mathrm{H}$, Tielenburg $\mathrm{R}$, and Mans A. Error detection during VMAT delivery using EPID-based 3D transit dosimetry. Physica Medica, 2018. 54: p. 137-145.

17. Piron O, Varfalvy N, and Archambault L. Establishing action threshold for change in patient anatomy using EPID gamma analysis and PTV coverage for head and neck radiotherapy treatment. Medical Physics, 2018. 45(8): p. 3534-3545.

18. Zhuang AH and Olch AJ. Sensitivity study of an automated system for daily patient QA using 
EPID exit dose images. Journal of Applied Clinical Medical Physics, 2018. 19(3): p. 114-124.

19. Olaciregui-Ruiz I, Rozendaal R, Kranen S, Mijnheer B, and Mans A. The effect of the choice of patient model on the performance of in vivo 3D EPID dosimetry to detect variations in patient position and anatomy. Medical Physics, 2019.

20. Olaciregui-Ruiz I, Rozendaal R, Mijnheer B, and Mans A. Site-specific alert criteria to detect patient-related errors with 3D EPID transit dosimetry. Medical Physics, 2019. 46(1): p. 4555.

21. Varfalvy N, Piron O, Cyr MF, Dagnault A, and Archambault L. Classification of changes occurring in lung patient during radiotherapy using relative $\gamma$ analysis and hidden Markov models. Medical Physics, 2017. 44(10): p. 5043-5050.

22. Nyflot MJ, Thammasorn P, Wootton LS, Ford EC, and Chaovalitwongse WA. Deep learning for patient-specific quality assurance: Identifying errors in radiotherapy delivery by radiomic analysis of gamma images with convolutional neural networks. Medical Physics, 2019. 46(2): p. 456-464.

23. Langendijk JA, Lambin P, De Ruysscher D, Widder J, Bos M, and Verheij M. Selection of patients for radiotherapy with protons aiming at reduction of side effects: The modelbased approach. Radiotherapy and Oncology, 2013. 107(3): p. 267-273.

24. Widder J, van der Schaaf A, Lambin P, Marijnen CAM, Pignol J-P, Rasch CR, Slotman BJ, Verheij $M$, and Langendijk JA. The quest for evidence for proton therapy: Model-based approach and precision medicine. International Journal of Radiation Oncology • Biology • Physics, 2016. 95(1): p. 30-36.

25. Längkvist $M$, Karlsson $L$, and Loutfi A. A review of unsupervised feature learning and deep learning for time-series modeling. Pattern Recognition Letters, 2014. 42: p. 11-24.

26. Yu D and Deng L. Deep neural network-Hidden Markov Model hybrid systems, in Automatic speech recognition: A deep learning approach. 2015, Springer London: London. p. 99-116.

27. Persoon LCGG, Podesta M, Hoffmann L, Sanizadeh A, Schyns LEJR, de Ruiter BM, Nijsten SMJJG, Muren LP, Troost EGC, and Verhaegen F. Is integrated transit planar portal dosimetry able to detect geometric changes in lung cancer patients treated with volumetric modulated arc therapy? Acta Oncologica, 2015. 54(9): p. 1501-07.

28. Persoon LCGG, Podesta M, Nijsten SMJJG, Troost EGC, and Verhaegen F. Time-resolved versus integrated transit planar dosimetry for volumetric modulated arc therapy: Patient-specific dose differences during treatment, a proof of principle. Technology in Cancer Research \& Treatment, 2015. 15(6): p. NP79-87.

29. Valdes G, Chan MF, Lim SB, Scheuermann R, Deasy JO, and Solberg TD. IMRT QA using machine learning: A multi-institutional validation. Journal of Applied Clinical Medical Physics, 2017. 18(5): p. 279-284.

30. Interian Y, Rideout V, Kearney VP, Gennatas E, Morin O, Cheung J, Solberg T, and Valdes G. Deep nets vs expert designed features in medical physics: An IMRT QA case study. Medical Physics, 2018. 45(6): p. 2672-2680.

31. Tomori S, Kadoya N, Takayama Y, Kajikawa T, Shima K, Narazaki K, and Jingu K. A deep learningbased prediction model for gamma evaluation in patient-specific quality assurance. Medical Physics, 2018. 45(9): p. 4055-4065.

32. Lam D, Zhang X, Li H, Deshan Y, Schott B, Zhao T, Zhang W, Mutic S, and Sun B. Predicting gamma passing rates for portal dosimetry-based IMRT QA using machine learning. Medical Physics, 2019. 46(10): p. 4666-4675.

33. Kalantzis G, Vasquez-Quino LA, Zalman T, Pratx G, and Lei Y. Toward IMRT 2D dose modeling using artificial neural networks: A feasibility study. Medical Physics, 2011. 38(10): p. 58075817.

34. Chatrie F, Younan F, Mazurier J, Simon L, Vieillevigne L, Ferrand R, Barbeiro AR, Le Lann $\mathrm{M}-\mathrm{V}$, and Franceries $\mathrm{X}$. Electronic portal imaging devices using artificial neural networks. Presented 2019. Singapore: Springer Singapore. 
35. Mijnheer B. EPID-based dosimetry and its relation to other 2D and 3D dose measurement techniques in radiation therapy. Journal of Physics: Conference Series, 2017. 847: p. 012024.

36. Javaid U, Souris K, Dasnoy D, Huang S, and Lee JA. Mitigating inherent noise in Monte Carlo dose distributions using dilated U-Net. Medical Physics, 2019. 46(12): p. 5790-5798.

37. Bibault J-E, Giraud P, and Burgun A. Big Data and machine learning in radiation oncology: State of the art and future prospects. Cancer Letters, 2016. 382(1): p. 110-117.

38. Thompson RF, Valdes G, Fuller CD, Carpenter CM, Morin O, Aneja S, Lindsay WD, Aerts $\mathrm{HJ}$, Agrimson B, and Deville C. Artificial intelligence in radiation oncology imaging. International Journal of Radiation Oncology • Biology • Physics, 2018. 102(4): p. 1159-1161.

39. Thompson RF, Valdes G, Fuller CD, Carpenter CM, Morin O, Aneja S, Lindsay WD, Aerts HJ, Agrimson B, and Deville Jr C. Artificial intelligence in radiation oncology: A specialty-wide disruptive transformation? Radiotherapy and Oncology, 2018. 129(3): p. 421-426.

40. Tang X. The role of artificial intelligence in medical imaging research. BJR|Open, 2020. 2(1): p. 20190031.

41. Jia X, Ren L, and Cai J. Clinical implementation of Al technologies will require interpretable AI models. Medical Physics, 2020. 47(1): p. 1-4.

42. Mijnheer B. EPIDs and QA of advanced treatments. Journal of Physics: Conference Series, 2019. 1305: p. 012061. 


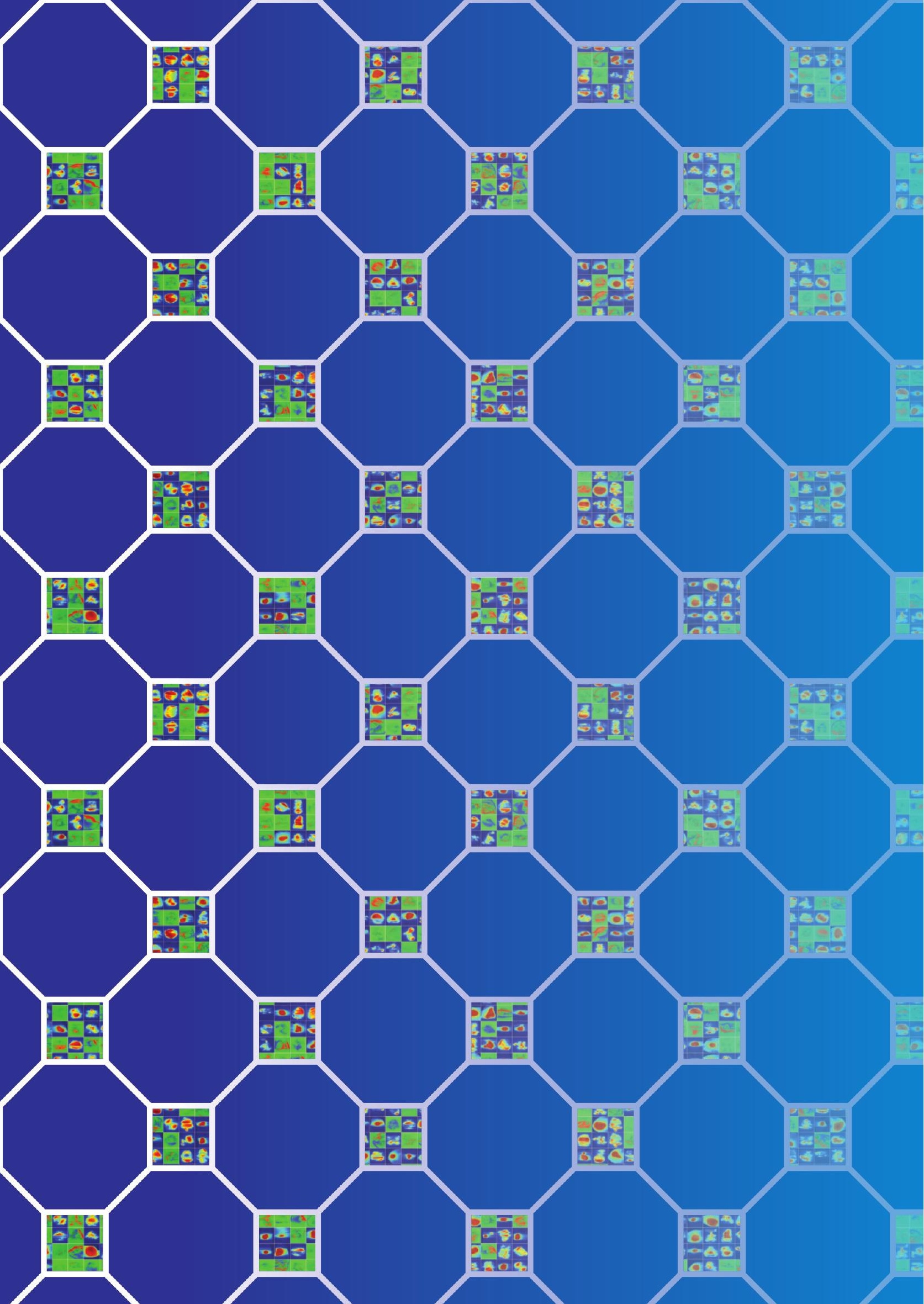




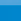





\section{Summary}

Radiotherapy is one of the main treatment options for many cancer types. Over the years, radiotherapy treatment delivery has become more precise and complex, with the aim of delivering a high radiation dose to the tumor and as little dose as possible to surrounding healthy tissues. With the increasing complexity of radiotherapy, it has become increasingly important to verify that the desired radiation dose is delivered as planned. This thesis focuses on dose-guided radiotherapy (DGRT) using portal dosimetry with the electronic portal imaging device (EPID) as a method for treatment verification. Measured dose distributions with the EPID can be compared to planned dose distributions, such that relevant deviations in the treatment can be detected. The aim is to flag treatments in which relevant dosimetric errors occur, such that the treatment can be adapted. However, automatic error detection and consequently patient selection for adaptive radiotherapy using portal dosimetry remains difficult. One reason for this is the unknown uncertainty of the portal dosimetry methods, while another reason is the lack of objective triggers for adaptation and the use of simple decision protocols (often consisting of an action threshold on a single metric extracted from dose comparisons) that cannot take the multidimensionality of the EPID dose distributions into account.

The overall aim of this thesis was to investigate and improve the error detection capabilities of portal dosimetry methods. To this end, the thesis is divided into two parts. The first part concerns uncertainties and sensitivity of portal dosimetry methods. In Chapter 2, a framework for analyzing uncertainties of portal dosimetry methods was established and this framework was applied to a larger dataset in Chapter 3, showing that the uncertainty is influenced by the presence of small radiation fields, which are becoming more common as treatment complexity increases. Therefore, it is important to incorporate this uncertainty in decision protocols for adaptive radiotherapy. The sensitivity and specificity of several portal dosimetry methods for detecting simulated anatomical changes using a simple threshold based classification method was investigated in Chapter 4, where it was shown that different combinations of portal dosimetry methods, comparison criteria and action thresholds are optimal for detecting different types of errors. This differs from current clinical practice, where the same comparison criteria and action threshold are used for all cases.

The second part of this thesis focuses on advanced methods for error detection and classification, applying artificial intelligence ( $\mathrm{Al}$ ) to portal dosimetry. $\mathrm{Al}$ techniques can take more information into account than the currently used action threshold classification methods. Several Al methods and classification problems were investigated to show the potential of Al-based error detection for portal dosimetry. Chapter 5 provides a proof of concept of a deep learning model for detecting relevant dose errors in 2D dose comparisons based on portal dosimetry simulations. This shows that it is possible to relate $2 \mathrm{D}$ information to differences in metrics based on the $3 \mathrm{D}$ dose in the target structure by using a convolutional neural network (CNN). This relationship between $2 \mathrm{D}$ and $3 \mathrm{D}$ dose information is notoriously difficult to find 
with traditional action threshold based classification methods. In Chapter 6, this work was extended to portal dosimetry measurements obtained during patient treatment, and includes dose metrics for organs-at-risk (OARs) as well. Here it was also possible to relate 2D dose comparisons to metrics extracted from 3D dose distributions, although generalizability of the model to new data is an issue that needs to be addressed in future work. Chapter $\mathbf{7}$ took a different approach, as the aim was to classify the type of error that occurred. This simulation study showed that deep learning is a promising powerful tool for detecting types of treatment errors with portal dosimetry. Errors can be detected to a high level of detail, and this approach can provide additional information not currently available from portal dosimetry in clinical practice. In Chapter 8, a machine learning model for classifying severity of anatomical changes developed at CHU de Québec (Canada) was validated using portal dosimetry data from Maastro. This study showed that it is difficult to use such a model on clinical data from a different institute due to differences in data acquisition and clinical interpretation. There was, however, a relationship between model classification (which was based on metrics extracted from 2D dose comparisons) and differences in 3D dose metrics for certain OARs, which can be useful for selecting patients that may need dose recalculation or adaptation of their treatment plan.

Overall, portal dosimetry for DGRT can greatly benefit from improved error detection methods. This thesis has contributed to this improvement, by providing a framework for uncertainty analysis of portal dosimetry methods, by examining the sensitivity and specificity for action threshold based classification methods for various portal dosimetry methods, and by investigating various advanced Al algorithms and error classification problems. 


\section{Samenvatting}

Radiotherapie is een belangrijke behandeloptie voor veel kankertypes. In de loop der jaren is de toediening van radiotherapiebehandelingen nauwkeuriger en complexer geworden, met als doel het toedienen van een hoge stralingsdosis aan de tumor en zo min mogelijk aan omliggende gezonde weefsels. Met de toenemende complexiteit van radiotherapie, wordt het steeds belangrijker om te verifiëren dat de gewenste stralingsdosis wordt afgeleverd zoals gepland. Dit proefschrift richt zich op dosis-gestuurde radiotherapie (DGRT) met behulp van dosimetrie met elektronische röntgencamera's (electronic portal imaging device: EPID) als methode voor behandelverificatie. Gemeten dosisverdelingen met de EPID kunnen worden vergeleken met geplande dosisverdelingen, zodat relevante afwijkingen in de behandeling kunnen worden opgespoord. Het doel is behandelingen te identificeren waarbij relevante dosimetrische fouten optreden, zodat de behandeling kan worden aangepast. Automatische foutdetectie met deze verificatie methode, en daardoor patiëntselectie voor adaptieve radiotherapie, blijft een probleem. Eén reden hiervoor is de onbekende onzekerheid van de EPID dosimetrie methoden, terwijl een andere reden het gebrek aan objectieve grenswaarden voor aanpassing van de behandeling is en daarbij het gebruik van eenvoudige beslisprotocollen (vaak bestaand uit een drempelwaarde op een enkele variabele, afgeleid van dosisvergelijkingen) die geen rekening kunnen houden met de multi-dimensionaliteit van de EPID-dosisverdelingen.

Het doel van dit proefschrift was het onderzoeken en verbeteren van foutdetectie met EPID dosimetrie methoden. Daartoe is het proefschrift verdeeld in twee delen. Het eerste deel betreft de onzekerheden en sensitiviteit van EPID dosimetrie methoden. In Hoofdstuk 2 werd een kader voor het analyseren van onzekerheden van EPID dosimetrie methoden opgesteld en dit kader werd toegepast op een grotere dataset in Hoofdstuk 3, waarin werd aangetoond dat de onzekerheid wordt beïnvloed door de aanwezigheid van kleine stralingsvelden, die steeds vaker voorkomen naarmate de complexiteit van de behandeling toeneemt. Het is daarom belangrijk om deze onzekerheid op te nemen in beslisprotocollen voor adaptieve radiotherapie. De sensitiviteit en specificiteit van verschillende EPID dosimetrie methoden voor het opsporen van gesimuleerde anatomische veranderingen met behulp van een eenvoudige classificatiemethode gebaseerd op drempelwaarden werd onderzocht in Hoofdstuk 4. Daar werd aangetoond dat verschillende combinaties van EPID dosimetrie methoden, vergelijkingscriteria en drempelwaarden optimaal zijn voor het detecteren van verschillende typen fouten. Dit verschilt van de huidige klinische praktijk, waar voor alle gevallen dezelfde vergelijkingscriteria en drempelwaarde worden gebruikt.

Het tweede deelvan het proefschrift richtzich opgeavanceerdemethoden voor foutdetectie en classificatie, waarbij kunstmatige intelligentie (KI) wordt toegepast op EPID dosimetrie. KI technieken kunnen meer informatie verwerken dan de momenteel gebruikte classificatiemethoden gebaseerd op drempelwaarden. Verschillende KI methoden en classificatieproblemen werden onderzocht om de potentie van op KI 
gebaseerde foutdetectie voor EPID dosimetrie aan te tonen. Hoofdstuk $\mathbf{5}$ biedt een 'proof of concept' van een deep learning model voor het detecteren van relevante dosisfouten in 2D dosisvergelijkingen op basis van EPID dosimetrie simulaties. Dit laat zien dat het mogelijk is om 2D informatie te relateren aan verschillen in variabelen afgeleid van de 3D dosis in de doelstructuur/tumor met behulp van een convolutioneel neuraal netwerk (CNN). Deze relatie tussen 2D en 3D dosisinformatie is moeilijk te vinden met traditionele classificatiemethoden gebaseerd op drempelwaarden. In Hoofdstuk 6 werd dit werk uitgebreid met EPID dosimetrie metingen die werden verkregen tijdens de behandeling van patiënten, en zijn variabelen voor risicoorganen (organs-at-risk: OARs) ook meegenomen. Hier was het ook mogelijk om 2D dosisvergelijkingen te relateren aan variabelen afgeleid van 3D dosisverdelingen, hoewel de generaliseerbaarheid van het model naar nieuwe data een probleem is dat in de toekomst verder moet worden onderzocht. Hoofdstuk 7 gebruikte een andere aanpak, waarbij het doel was om het type fout dat zich voordeed te classificeren. Deze simulatiestudie toonde aan dat deep learning een veelbelovend krachtig hulpmiddel is voor het detecteren van verschillende soorten behandelingsfouten met EPID dosimetrie. Fouten kunnen tot in detail worden opgespoord en deze werkwijze kan aanvullende informatie opleveren die momenteel in de klinische praktijk niet beschikbaar is vanuit EPID dosimetrie. In Hoofdstuk $\mathbf{8}$ werd een machine learning model voor het classificeren van de ernst van anatomische veranderingen, ontwikkeld aan CHU de Québec (Canada), gevalideerd met behulp van EPID dosimetrie metingen van Maastro. Deze studie toonde aan dat het moeilijk is om dit model te gebruiken op klinische data van een ander instituut, te wijten aan verschillen in data acquisitie en klinische interpretatie. Er was echter een verband tussen modelclassificatie (die was gebaseerd op variabelen afgeleid van $2 \mathrm{D}$ dosisvergelijkingen) en verschillen in variabelen afgeleid van de 3D dosis voor bepaalde OARs, wat nuttig kan zijn voor selectie van patiënten die mogelijk een dosisherberekening of aanpassing van hun behandelplan nodig hebben.

In het algemeen kan EPID dosimetrie voor DGRT aanzienlijk voordeel halen uit verbeterde methoden voor foutdetectie. Dit proefschrift heeft bijgedragen aan deze verbetering door een kader te bieden voor analyse van onzekerheden van EPID dosimetrie methoden, door de sensitiviteit en specificiteit te onderzoeken van classificatiemethoden gebaseerd op drempelwaarden voor verschillende EPID dosimetrie methoden, en door verschillende geavanceerde $\mathrm{KI}$ algoritmen en foutclassificatie problemen te onderzoeken. 


\section{Valorization Addendum}

\section{Relevance}

Cancer has been and will remain a prominent disease worldwide. Over the years, treatment options have improved substantially and quickly. In the field of radiotherapy, technological advancements have driven the development of more conformal, but also more complex treatment. These advancements have intensified the need for accurate treatment verification, such that treatment errors can be detected and corrected, avoiding potential ineffective treatment or harm to patients. Additionally, in vivo error detection methods can be employed for adaptive radiotherapy, in which a patient's treatment plan is adjusted when persisting errors occur, personalizing radiotherapy treatment even further. In several countries, in vivo verification of the treatment has even become mandatory.

Portal dosimetry using the electronic portal imaging device (EPID) has proven to be a suitable and flexible candidate for verification of complex treatments. It can be used in different stages of the radiotherapy workflow (i.e., pre-treatment and in vivo) as well as provide dose distributions in various dimensions (i.e., 2D, 2D+time, 3D and 4D), which is necessary for increasingly complex treatments. Using portal dosimetry, the true dose received by the patient can be recorded. With the addition of the methods described in this thesis, portal dosimetry can become an even more effective method for verifying radiotherapy treatment and detecting errors, thereby improving overall patient treatment.

\section{Products, innovation and target groups}

Over the years, multiple vendors have invested in the development of EPID-based portal dosimetry systems. This resulted in commercially available systems with different capabilities, ranging from point dose verification (e.g., SOFTDISO (Best Medical, Italy)), to 2D (e.g., SunCHECK (Sun Nuclear, Melbourne, FL, USA)) and 3D dose verification (e.g., Dosimetry Check (LifeLine Software, Inc., Austin, TX, USA)) or a combination thereof (e.g., 2D and 3D with iViewDose (Elekta, Stockholm, Sweden)). None of these solutions currently offer time-resolved dose verification. The big advantage of the EPID is that it is widely available as it comes with most linacs, hence, no additional hardware is needed. Nevertheless, the EPID was not originally designed for dosimetry, which necessitates calibration and complex models for acquiring dose distributions, which the aforementioned software packages facilitate.

A general issue that vendors of portal dosimetry systems face, is that portal dosimetry is underutilized in radiotherapy clinics. In 2017, a European Society for Radiotherapy and Oncology (ESTRO) Task Group for in vivo dosimetry was established, with the aim of identifying the main reasons for the clinical underutilization of in vivo dosimetry methods (with a focus on EPID-based portal dosimetry) and to define requirements for in vivo dosimetry systems necessary for increased clinical adoption. This shows that there is a general interest in the radiotherapy community for advancing the field of EPID-based portal dosimetry, but that there are still some hurdles that need 
to be taken. These hurdles are related to various aspects of portal dosimetry, such as automation of all parts of the workflow, understanding and reduction of uncertainties, interpretation of results and improvement of error detection. The methods presented in this thesis overcome some of these hurdles, especially those related to uncertainties and interpretation of results for improved error detection.

One of the reasons for the underutilization of portal dosimetry is that the uncertainties, sensitivity and specificity of these methods are often unknown. Moreover, interpretation of the results remains difficult as multi-dimensional information obtained with portal dosimetry is usually reduced to a few metrics to remain manageable for human interpreters. The research in this thesis fills these gaps and proposes novel methods for solving these issues, making portal dosimetry an even more attractive method for treatment verification and potentially increasing its clinical utilization.

To increase the clinical employment of portal dosimetry systems, their error detection capabilities need to be systematically investigated, quantified and reported. On the one hand, this implies analyzing the uncertainties, sensitivity and specificity of implemented portal dosimetry methods, as was done in the first part of this thesis. Only if vendors of portal dosimetry software invest in uncovering these aspects for their portal dosimetry solution in similar manners, and clearly report their findings, can utilization of portal dosimetry be improved. The capabilities and limitations of portal dosimetry systems need to be clear to the clinical users, and the methods presented in part I of this thesis can be used as a framework for quantifying these.

On the other hand, sophisticated methods for interpretation of portal dosimetry results are needed. Vast amounts of data are produced when portal dosimetry is used in a radiotherapy clinic, necessitating automated methods for analyzing these results and detecting relevant deviating cases. Currently, analyzing portal dosimetry results is often a task of medical physicists, who have to manually assess flagged cases. However, as simple threshold methods based on reduced information are used for flagging, there are many false positive cases, and an unknown number of false negatives. With the more sophisticated methods developed in part II of this thesis, the number of false positive and false negative cases will be reduced, improving clinical workload and minimizing the risk of missing relevant deviations.

The application of artificial intelligence (AI) for classification of errors in portal dosimetry is a novel topic, where many aspects still have to be explored. However, part II of this thesis shows that it is a promising road to follow. Advanced methods for error detection as described in this thesis should be added to existing portal dosimetry software packages, although more external validation is needed first, to ensure generalizability and transferability of these methods to different radiotherapy institutes. Underlying differences in clinical practice can make it difficult to provide one model that will be relevant for many different radiotherapy institutes. Although this may be an issue, it also provides an opportunity for standardizing criteria for error detection among centers and removing subjectivity from this process.

An ideal portal dosimetry solution should include Al models for error classification, of which the error detection capabilities have been quantified, such that it is immediately clear to the clinical user what the uncertainties of the methods are, and which errors can be detected and which cannot. Such plug-and-play solutions 
will increase the interest of the radiotherapy community in portal dosimetry, as they will provide a straightforward way of verifying and improving complex radiotherapy treatments, saving time and workload compared to current portal dosimetry solutions. This thesis is among the first to study the application of Al models for error detection in portal dosimetry, and a vast amount of work remains to be done. Further development of these models towards an ideal portal dosimetry solution also requires effort from vendors, as well as clinical users and international societies such as ESTRO.

The research in this thesis has been performed within multiple fruitful research agreements with Varian Medical Systems (Palo Alto, CA, USA); one of the largest manufacturers of medical radiotherapy devices and software. In previous research agreements, portal dosimetry methods were developed and several of these models have been acquired by Varian. This acquisition and the continued research collaboration demonstrates the interest of a large player in the radiation oncology field in these treatment verification methods, which will lead to the development of a commercial product for portal dosimetry based on the research performed at Maastro. This thesis is part of this ongoing collaboration, and evaluates aspects of portal dosimetry that are needed for making a successful commercial product. These aspects are quantification of the error detection capabilities of the portal dosimetry methods and development of novel classification methods for improved error detection. Even though the results of this thesis are specific for Varian linacs and EPIDs, the methods can be extended and applied to linacs and EPIDs of other vendors.

Although radiotherapy is already a safe treatment option, detection of errors, even if they are small, enhances treatment quality and provides potential for adaptive radiotherapy, hence increased personalization of treatment. Ultimately, an advanced portal dosimetry solution should and will benefit patients, as errors in their radiotherapy treatment can reliably be detected and corrected, preventing underdosing the tumor and/or overdosing healthy tissues. This is in line with one of the goals of Maastro (Maastricht, The Netherlands), where this research was performed, which is "to reduce the chance of side effects and recurrence of the tumor, through the use of, among others, dose-guided adaptive radiotherapy". 



\section{Curriculum Vitae}

Cecile Julie Andrée Wolfs was born on January 17, 1991 in Maastricht, The Netherlands. After finishing her secondary education at the Bonnefanten College in Maastricht in 2009, she pursued a bachelor's degree in Liberal Arts and Sciences at University College Maastricht (UCM) of Maastricht University. At UCM, her major was in mathematics, with a minor in computer science. During her third year, she studied abroad at the University of Richmond (Richmond, Virginia, USA) for one semester. After returning, she wrote her bachelor's thesis on the topic of evolutionary game theory, and its application in the biomedical domain. She graduated from UCM with honors in July 2012.

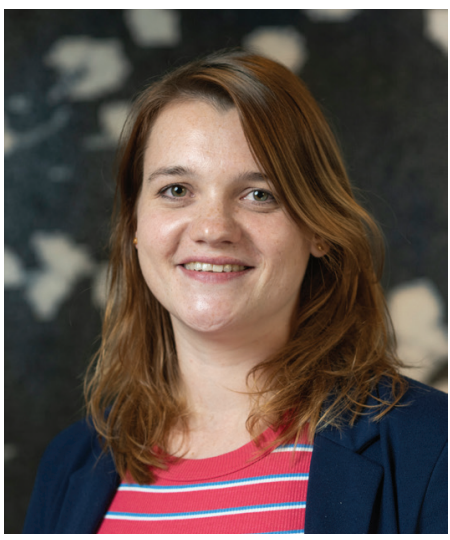

In September 2012, she started a master's program in Operations Research at the Department of Knowledge Engineering of Maastricht University. During this master's program, she did a research internship at the Maastricht University Medical Centre+, concerning optimization of operating room planning. The topic of her master's thesis was early detection of adverse cardiac events, using ECG signals from implanted cardiac devices. She graduated in July 2015.

In April 2016 she started her PhD at Maastro, in the Physics Research group under the supervision of prof. dr. Frank Verhaegen. Her research concerned error detection in dose-guided radiotherapy using portal dosimetry. During the first years of her PhD, she focused on uncovering uncertainties in the complex portal dosimetry chain, and quantifying the sensitivity and specificity of portal dosimetry methods. Halfway through her $\mathrm{PhD}$, her colleagues and she obtained a research grant from Varian Medical Systems, to continue their research on error detection in dose-guided radiotherapy using portal dosimetry, and extending it to include artificial intelligence. This project was the focus of the second half of her PhD, and she will now continue this work as a postdoctoral researcher at Maastro.

\section{Personal grants}

- NRS Young Investigator Travel Grant to attend Biology-Guided Adaptive Radiotherapy (BiGART) in Aarhus, Denmark, May 2017

- ESTRO Mobility Grant/Technology Transfer Grant for a research visit to CHU de Québec/Université Laval, Québec, QC, Canada, June 2019

- NRS Young Investigator Travel Grant to attend International Conference on the use of Computers in Radiation therapy (ICCR) in Montréal, QC, Canada, June 2019

\section{Extracurricular activities}

- PhD Representative at GROW School for Oncology, Maastricht University Medical Centre+ (October 2017 - January 2020) 



\section{List of publications}

\section{Peer-reviewed articles}

F. Verhaegen, R.-G. Wanders, C.J.A. Wolfs and D. Eekers. TRANSIT-FLASH; a novel concept for proton therapy. (2020) Under review.

C.J.A. Wolfs, R.A.M. Canters and F. Verhaegen. Detection of treatment error types using convolutional neural networks and EPID dosimetry. (2020) Under review.

A. Vaniqui, R.A.M. Canters, F. Vaassen, C. Hazelaar, I. Lubken, K. Kremer, C.J.A. Wolfs and W. van Elmpt. Treatment plan quality assessment for radiotherapy of rectal cancer patients using prediction of organ-at-risk dose metrics. (2020) Under review.

C.J.A. Wolfs, N. Varfalvy, R.A.M. Canters, S.M.J.J.G. Nijsten, L. Archambault* and F. Verhaegen*. External validation of a hidden Markov model for gamma-based classification of anatomical changes in lung cancer patients using EPID dosimetry. Accepted for publication in Medical Physics (2020).

C.J.A. Wolfs, A.C.C. Swinnen, S.M.J.J.G. Nijsten and F. Verhaegen. Should dose from small fields be limited for dose verification procedures?: uncertainty versus small fields dose in VMAT treatments. Physics in Medicine and Biology (2018) 63(20) 20NT01

J.A. Baeza*, C.J.A.Wolfs*, S.M.J.J.G. Nijsten and F. Verhaegen. Validation and uncertainty analysis of a pre-treatment 2D dose prediction model. Physics in Medicine and Biology (2018) 63(3) 035033

C.J.A. Wolfs*, M.G. Brás*, L.E.J.R. Schyns, S.M.J.J.G. Nijsten, W. van Elmpt, S.G. Scheib, C. Baltes, M. Podesta and F. Verhaegen. Detection of anatomical changes in lung cancer patients with 2D time-integrated, 2D time-resolved and 3D time-integrated portal dosimetry: a simulation study. Physics in Medicine and Biology (2017) 62(15) 6044-61

\section{Other scientific publications}

C.J.A. Wolfs. Mobility grant report: EPID dosimetry and adaptive radiotherapy with artificial intelligence. Young Corner, ESTRO Newsletter. October 2019.

C.J.A. Wolfs, B. van der Heyden and F. Verhaegen. Deep learning for EPID dosimetry: A proof of concept. Conference proceedings of the ICCR2019. June 2019.

* These authors contributed equally 


\section{Conference abstracts, posters and presentations}

C.J.A. Wolfs, N. Varfalvy, R.A.M. Canters, S.M.J.J.G. Nijsten, L. Archambault and F. Verhaegen. Validating a hidden Markov model for lung anatomical change classification using EPID dosimetry. Poster highlight at ESTRO 2020; Vienna, Austria; November 2020.

G.P. Fonseca, I.P. Almeida, T. van Wagenberg, C.J.A. Wolfs, G. Vilches Freixas, I. Rinaldi, J. Martens, G. Bosmans and F. Verhaegen. An independent platform for dose calculation and log file evaluation in proton therapy. Poster highlight at ESTRO 2020; Vienna, Austria; November 2020.

A. Vaniqui, R.A.M. Canters, F. Vaassen, C. Hazelaar, I. Lubken, K. Kremer, C.J.A. Wolfs and W. van Elmpt. Plan quality assessment for rectal cancer patients using prediction of organ-at-risk dose metrics. Poster discussion at ESTRO 2020; Vienna, Austria; November 2020.

M. Alahmari, C.J.A. Wolfs, Y. Temel, D. Eekers and F. Verhaegen. Comparing dose calculation algorithms in low dose regions for patients with brain metastases. E-Poster at ESTRO 2020; Vienna, Austria; November 2020.

C.J.A. Wolfs, N. Varfalvy, R.A.M. Canters, S.M.J.J.G. Nijsten, L. Archambault and F. Verhaegen. External validation of a machine learning model for classification of anatomical changes in lung cancer patients. Poster presentation at GROW Science Day; Maastricht, The Netherlands; November 2019.

C.J.A. Wolfs, N. Varfalvy, R.A.M. Canters, S.M.J.J.G. Nijsten, L. Archambault and F. Verhaegen. External validation of a hidden Markov model for lung anatomical change classification using EPID dosimetry. Oral presentation at RKF Scientific Project Day; Rotterdam, The Netherlands; November 2019.

C.J.A. Wolfs, B. van der Heyden and F. Verhaegen. Deep learning for EPID dosimetry: A proof of concept. Poster presentation at ICCR2019; Montréal, Canada; June 2019.

K. Surmann, J.A. Baeza, C.M.L. Zegers, C.J.A. Wolfs, S.M.J.J.G. Nijsten, M.C. Oellers, B. Reymen and F. Verhaegen. Anatomical changes and patient selection for dose-guided adaptive radiotherapy in lung cancer patients. Poster presentation at Biology-Guided Adaptive Radiotherapy (BiGART) 2019; Aarhus, Denmark; May 2019.

C.J.A. Wolfs, A.C.C. Swinnen, S.M.J.J.G. Nijsten and F. Verhaegen. Should dose from small fields be limited for dose verification procedures?: uncertainty versus small fields dose in VMAT treatments. Oral presentation at RKF Scientific Project Day; Utrecht, The Netherlands; January 2019.

C.J.A. Wolfs, B. van der Heyden and F. Verhaegen. Artificial intelligence based correction of daily radiotherapy treatment of lung cancer patients. Poster presentation at GROW Science Day; Maastricht, The Netherlands; November 2018. 
C.J.A. Wolfs, S.M.J.J.G. Nijsten and F. Verhaegen. Relation between uncertainty and dose from small fields for a 2D pre-treatment dose prediction model. Radiotherapy and Oncology 127 (2018): S982-S983. E-poster at ESTRO37; Barcelona, Spain; April 2018.

J.A. Baeza*, C.J.A.Wolfs*, S.M.J.J.G. Nijsten and F.Verhaegen. Validation and uncertainty analysis of a pretreatment prediction model for EPID dosimetry. Poster at BiologyGuided Adaptive Radiotherapy (BiGART) 2017; Aarhus, Denmark; June 2017.

C.J.A. Wolfs, M.G. Brás, M. Gil Conde, L.E.J.R. Schyns, S.M.J.J.G. Nijsten, M. Podesta and F. Verhaegen. Dosimetric consequences of simulated anatomical changes in lung cancer patients. Poster at Biology-Guided Adaptive Radiotherapy (BiGART) 2017; Aarhus, Denmark; June 2017.

C.J.A. Wolfs, M. Podesta, L.E.J.R. Schyns, M.G. Brás, L.C.G.G. Persoon, S.G. Scheib, C. Baltes and F. Verhaegen. Detecting relevant anatomical changes in lung cancer patients with EPID dosimetry. Poster at Varian Research Symposium 2017; Chicago II, United States of America; February 2017. 



\section{Dankwoord}

Daar zijn we dan, aan het einde van mijn proefschrift en het einde van een intensieve periode van ruim 4 jaar. Een periode die zeker niet altijd makkelijk is geweest, maar die me ook heel veel mooie herinneringen en vriendschappen heeft gebracht. Zoals velen van jullie weten is een $\mathrm{PhD}$ afronden niet iets dat je in je eentje doet, en zijn er dus veel mensen die ik hier graag wil bedanken voor hun bijdrage aan mijn promotie.

Allereerst wil ik mijn promotieteam bedanken, bestaande uit prof. dr. ir. Frank Verhaegen, dr. Gabriel Paiva Fonseca en dr. ir. Bas Nijsten.

Frank, $u$ heeft een gok genomen toen $u$ mij, een net afgestudeerde masterstudent zonder achtergrond in fysica, aannam. Ik ben erg blij dat deze gok goed heeft uitgepakt, en dat ik op deze manier in de wereld van de klinische fysica terecht gekomen ben. $U$ heeft altijd de tijd genomen om mij nieuwe dingen te leren, en omgekeerd stond $u$ ook open om zelf nieuwe dingen te leren, wat onder andere leidde tot een project waar onze achtergronden en interesses in gecombineerd konden worden. Daarnaast heeft $u$ altijd begrip gehad voor mijn persoonlijke situatie, wat ik heel erg waardeer.

Gabriel, you are an integral part of Frank's team and my PhD. Even though we haven't co-authored any papers, you played an important role in finishing my PhD. I have said this before, but you truly are the superman of our team. Thank you for always answering my questions, helping me and providing guidance wherever possible.

Bas, jij hebt aan de basis gestaan van DGRT bij Maastro, en zonder jou zou mijn onderzoek niet eens bestaan hebben. Het is mooi om te zien dat je nog altijd enthousiast wordt over nieuwe ontwikkelingen op dit gebied, zoals de methoden die we in dit proefschrift onderzocht hebben. Ondanks je drukke agenda heb je mij toch altijd goed kunnen helpen, bedankt daarvoor.

I would like to thank the members of the assessment committee (prof. dr. ir. André Dekker, prof. dr. Dirk De Ruysscher, prof. dr. Eric Ford and prof. dr. Guillaume Landry), for reading and critically assessing my thesis.

To all my co-authors, thank you for the nice collaborations and your valuable input on my work.

Dr. Louis Archambault and dr. Nicolas Varfalvy, thank you for the time I got to spend at CHU de Québec. It was a very educational experience, that resulted in a publication and chapter in this thesis. I'm looking forward to continue our collaboration.

Dr. Christof Baltes and dr. Stefan Scheib from Varian Medical Systems, from the start of my PhD we had regular teleconferences, which were always very helpful. You always asked the right critical questions, which definitely improved my work. Thank you for that, and also for the enthusiasm you show for this line of research.

Met veel van de klinisch fysici, innovatie fysici en klifio's bij Maastro heb ik een prettige samenwerking en leuk contact gehad. In het bijzonder wil ik Ans, Esther, Richard en Wouter noemen. Bedankt voor alle hulp met metingen en data verzamelen. Daarnaast was het ook erg fijn dat ik regelmatig bij jullie aan kon sluiten voor de lunch! 
Ook alle andere Maastro afdelingen waar ik tijdens mijn PhD mee te maken heb gehad verdienen een bedankje: Academy, ICT, P\&O en het secretariaat. Jullie staan altijd open voor vragen en zijn erg betrokken. Sonia, jou wil ik nog extra bedanken voor al je hulp met de administratieve zaken rondom mijn promotie.

Daarnaast wil ik de Maastro yoga groep bedanken. Het was heel fijn om in de afrondende fase van mijn PhD elke maandagmiddag de week met een yogales bij Maastro te kunnen beginnen. Jennifer en Tjinta, bedankt voor de organisatie van deze lessen, en Ellen, bedankt voor het lesgeven!

During my PhD, I had the opportunity to become a PhD representative for GROW. I would like to thank my fellow PhD representatives (Niken, Eduardo, Kim, Nhan, Kees and Veronique) as well as the GROW office (Frans, Manon, Brigitte, Christel, Judith), PhD coordinators (Ton and Theo) and Young Investigators (Ludwig) for the pleasant collaboration. I am proud of how we evolved and became more involved during my time as representative, and I am sure you will continue this upwards trend.

Everyone I shared the Maastro research room with or met some other way during my PhD, thank you for the lunch breaks, (frequent) coffee breaks, barbecues, carnival celebrations, PVM activities, parties, ladies' nights, sports classes, but most of all, thank you for your company: Abdalla, Alberto, Alex, Ananya, Aniek, Anshu, Audrey, Behzad, Biche, Brent, Carolina, Damienne, Dennis, Esther, Frank, Georgios, Giacomo, Hajar, Hans, Henry, Isabel, Iva, Ivan, Joana, Johan, José, Jurgen, Leonard, Lotte, Louise, Manon, Mariana, Matilde, Merle, Nadine, Nick, Petros, Ralph, Renate, Rianne, Rosa, Ruben, Sebastian, Sergey, Shane, Simon, Stefan, Stuti, Teun, Tim, Timo, Turkey, Vera, Yvonka, Zhenwei.

Ana, you're probably the nicest and most thoughtful person l've ever met. From my first week at Maastro, when there suddenly was a Journal Club paper printed on my desk, until my birthday this year, when there suddenly was a gift on my desk. Even though these may seem small things, they meant a lot to me. Thank you for being there for me over the past years!

Daniela, ook al zagen we elkaar de laatste jaren niet vaak, als je bij Maastro was had je altijd tijd ingepland voor een koffiepauze. Bedankt voor alle goede gesprekken en gezelligheid!

Karen, bedankt dat ik altijd bij je terecht kon, waar ik ook mee zat. Onze gesprekken werden meestal iets langer dan gepland, maar waren voor mij altijd erg waardevol.

Mark, thank you for your patience, help and guidance in the first years of my PhD. I definitely learned a lot from you.

Matthijs, bedankt voor alle pauzes en wandelingen, en de daarbij behorende gesprekken en advies, waar ik veel aan gehad heb.

Murillo, thank you for our conversations about knitting and Apple gadgets, all the chocolate you left on my desk whenever you felt like it, and for regularly checking in with me during the lockdown.

Evelyn, Janita en Relinde / Eef, Jens en Reliend / de andere 3 leden van Maasquatro / mijn 3 paranimfen. Ik ben enorm blij dat ik jullie heb leren kennen tijdens mijn $\mathrm{PhD}$, en zowel op werk- als privégebied hebben jullie me in alles bijgestaan. Evelyn en Janita, vanaf dag 1 hebben jullie mij opgenomen in de groep en al snel gingen we als Maastrio door het leven. Toen Relinde erbij kwam werden we Maasquatro en 
samen hebben we ontzettend veel leuke dingen meegemaakt. Van koffiepauzes, tot maandelijkse etentjes, tot lange weekenden weg naar Odense, Brugge, Porto en Zürich en congressen in Aarhus en Barcelona. We zijn er tot nu toe goed in geslaagd om dit ook allemaal te blijven doen na het afronden van PhDs, dus wat mij betreft wordt dat nu niet anders!

Evelyn, bedankt voor al je advies, je enthousiasme en je vermogen om altijd de laatste nieuwtjes te hebben. Ik vind het super leuk dat we nu nog samen mogen werken en ik hoop dat je de rest van je opleiding met veel plezier kan afronden.

Janita, bedankt voor je nuchterheid, je begrip en alle keren dat we samen hebben gekookt. Het is fijn om te zien dat je op je plek zit in Zwitserland, en hoe je dat aanpakt is zeker iets waar ik van kan leren.

Relinde, bedankt voor je humor en dat je altijd tijd voor me maakte. Ik vind het knap hoe je alles in je drukke leven weet te combineren, en ik weet zeker dat het afronden van je promotie helemaal goed gaat komen.

Voor de sportieve ontspanning wil ik ook de Rudermädels/Dames 8+ van MWC bedanken. Zowel op het water als op de kant is het altijd erg gezellig. De jaarlijkse weekenden in Florence zijn zeker onderdeel van de hoogtepunten tijdens mijn promotie traject.

Jil, Katja, Stella en Zahra, jullie ken ik al bijna, of zelfs al ruim 20 jaar. Ook al zien we elkaar niet zo heel vaak, als we elkaar wel zien is het altijd weer als vanouds. Bedankt voor die leuke momenten en jullie vriendschap door de jaren heen.

Niek, ook jou wil ik bedanken voor de ontzettend leuke tijd die we samen hebben gehad tijdens mijn promotie. Onze lange weekenden in Zwitserland, vakantie in Amerika maar ook alle andere leuke dingen dichter bij huis, zal ik niet snel vergeten. En je weet het hè, van jou verwacht ik wel dat je me vanaf nu aanspreekt met dr. Wolfs.

Dan zijn we aangekomen bij de familie, die zeker heel belangrijk is als steun tijdens een promotie traject. Aan beide kanten van de familie is het een bewogen jaar geweest, en daarom wil ik graag Clim en Ger noemen. Helaas zijn jullie niet meer bij ons. Jullie overlijden, kort na elkaar in het laatste jaar van mijn PhD, heeft mij opnieuw duidelijk gemaakt waarom ik onderzoek doe in dit vakgebied, en waarom onderzoek naar kanker zo belangrijk is.

Opa, ook jou zijn we verloren afgelopen jaar. Je vroeg altijd of ik al wist wanneer mijn feest zou zijn, en ook al ben je er fysiek niet meer, je bent er in gedachten zeker bij. Jij en oma hebben altijd interesse getoond in hoe het met mijn werk ging, ook al wisten jullie niet altijd precies wat ik deed. Oma, jouw beroemde uitspraak 'Es iech 't mer haol!' is er een waar veel promovendi zich in kunnen vinden, en deze heeft het daarom ook tot mijn stellingen gehaald. Bedankt voor alle fijne herinneringen en haw pin!

Bert, Suzanne, Emile en Eva, bedankt voor de belangstelling die jullie hebben getoond voor mij en mijn promotie. Bert, de stage die jij me hebt geholpen te vinden tijdens mijn master is voor mij een doorslaggevende factor geweest in de keuze om voor een PhD te gaan, dus daarvoor nog extra bedankt.

Catherine, Kenny en Jules, ook jullie hebben mijn promotie van dichtbij meegemaakt en zijn altijd geïnteresseerd en betrokken geweest bij de mijlpalen van mijn PhD. De ups en downs hebben jullie ook meegekregen en bij beiden zijn jullie er voor mij geweest. Bedankt daarvoor. 
Papa en mama, Joy en Josette, jullie hebben zeker een enorme bijdrage geleverd aan dit proefschrift. Het zijn geen makkelijke jaren geweest, maar ik kon altijd bij jullie terecht en jullie hebben mij veel dingen uit handen genomen zodat ik me op mijn werk kon richten en mijn energie daaraan kon besteden. Er zijn geen woorden om uit te drukken hoe dankbaar ik daarvoor ben, dus daarom hou ik het bij daank uuch en iech haw vaan uuch. 